\title{
Mode and implementation of a hybrid control system for a 2-mode hybrid electric vehicle
}

zhenhua Zhu

West Virginia University

Follow this and additional works at: https://researchrepository.wvu.edu/etd

Part of the Electro-Mechanical Systems Commons, and the Navigation, Guidance, Control, and

Dynamics Commons

\section{Recommended Citation}

Zhu, zhenhua, "Mode and implementation of a hybrid control system for a 2-mode hybrid electric vehicle" (2013). Graduate Theses, Dissertations, and Problem Reports. 8162.

https://researchrepository.wvu.edu/etd/8162

This Dissertation is protected by copyright and/or related rights. It has been brought to you by the The Research Repository @ WVU with permission from the rights-holder(s). You are free to use this Dissertation in any way that is permitted by the copyright and related rights legislation that applies to your use. For other uses you must obtain permission from the rights-holder(s) directly, unless additional rights are indicated by a Creative Commons license in the record and/ or on the work itself. This Dissertation has been accepted for inclusion in WVU Graduate Theses, Dissertations, and Problem Reports collection by an authorized administrator of The Research Repository @ WVU.

For more information, please contact researchrepository@mail.wvu.edu. 


\title{
MODE AND IMPLEMENTATION OF A HYBRID CONTROL SYSTEM FOR A 2-MODE HYBRID ELECTRIC VEHICLE
}

\author{
Zhenhua Zhu \\ Dissertation submitted \\ To the Benjamin M. Statler College of Engineering and Mineral Resources \\ at West Virginia University \\ in partial fulfillment of the requirements for the degree of \\ Doctor of Philosophy in Department of Mechanical Engineering \\ Scott Wayne, Ph.D., Chair \\ Nigel Clark, Ph.D. \\ Hailin Li, Ph.D. \\ Chris Atkinson, Ph.D. \\ Natalia Schmid, Ph.D. \\ Department of Mechanical Engineering \\ Morgantown, West Virginia \\ November 2013
}

Keywords: 2-Mode, hybrid electric vehicle, hybrid control, hybrid vehicle modeling Copyright 2013 [Zhenhua Zhu] 


\title{
ABSTRACT \\ Modeling and Implementation of a Hybrid Control System for a 2-Mode Hybrid Electric Vehicle
}

\author{
Zhenhua Zhu
}

Automotive transportation consumes more than $71 \%$ of the world's total petroleum energy. The vehicle will have to use less because it will take less than 30 years to consume the rest of the proven conventional oil reservoir if the fuel consumption keeps the current trend. One of the methods to improve the vehicle efficiency is to develop Hybrid Electric Vehicle (HEV). An HEV combines an internal combustion engine (ICE) and electric motor(s) powered by batteries or capacitors to achieve better fuel economy. Among the HEVs, 2-Mode HEV combines an ICE and two electric motors with two planetary gear sets and allows the vehicle to operate in two Electrically Variable Transmission (EVT) modes with better fuel economy in both low speed range and high speed range.

A prototype 2-Mode HEV powertrain was developed and included a 1.3 liter 4cylinder turbocharger diesel engine, a 2-Mode front wheel drive transmission and a 330 V $12.9 \mathrm{kWh}$ high voltage battery. A forward-looking quasi-static model of the 2-Mode HEV was developed and parameterized with bench test data to evaluate the vehicle performance and fuel economy. A rule-based hybrid control algorithm was implemented to achieve engine stop-start, transmission shifting, high voltage battery charge sustaining, optimal engine speed and torque control, vehicle propulsion and regenerative braking. The prototype 2-Mode HEV was tested on road to verify vehicle safety features, acceleration and braking capability, autocross time, towing capacity and combined city and highway drive capability. Local city and highway drive schedules comparable to the UDDS and HWFET drive schedules were developed to test the vehicle's fuel economy.

The vehicle on-road test reached 24.5/31.5 MPG fuel economy, which was 23.8\% higher than the mule vehicle, 19.0/26.0 MPG. The vehicle was tested on road to evaluate the loss of the performance. The vehicle achieved $16 \mathrm{~s}$ during the $0-60 \mathrm{mph}$ acceleration and $10 \mathrm{~s}$ during the $50-70 \mathrm{mph}$ acceleration, both of which were $1.4 \mathrm{~s}$ longer than the mule vehicle. The braking distance from 60-0 mph was $148.5 \mathrm{ft}$, which was the same as the mule vehicle. The vehicle demonstrated the same towing ability at constant $45 \mathrm{mph}$ speed with a $3.5 \%$ grade for 15 miles.

The 2-Mode hybrid powertrain with a turbocharger diesel engine demonstrated an alternative to sustain petroleum energy and reduce the petroleum oil dependence in automotive transportation with fuel economy improvement of $23.8 \%$, but a small acceleration capability loss. The 2-Mode hybrid electric vehicle combined the strength 
and load capacity of conventional automatic transmission with the increased fuel economy provided by the hybrid electric vehicle architecture. 


\section{ACRONYM LIST}

\begin{tabular}{|c|c|}
\hline $\mathrm{A} / \mathrm{C}$ & Air Conditioning \\
\hline ABS & Antilock Brake System \\
\hline $\mathrm{AC}$ & Alternating Current \\
\hline ACIM & AC Inductance Motor \\
\hline $\mathrm{ACU}$ & Auxiliary Control Unit \\
\hline $\mathrm{ADC}$ & Analogue Digital Converter \\
\hline ADVISOR & ADvanced VehIcle SimulatOR \\
\hline AMESim & $\begin{array}{l}\text { Advanced Modeling Environment for performing Simulations of engineering } \\
\text { systems }\end{array}$ \\
\hline APP & Accelerator Pedal Position \\
\hline ASM & Automotive Simulation Model \\
\hline ASME & American Society of Mechanical Engineers \\
\hline AVL & Anstalt für Verbrennungskraftmaschinen List \\
\hline BAS & Belt Alternator Starter \\
\hline $\mathrm{BEV}$ & Battery Electric Vehicle \\
\hline BMS & Battery Management System \\
\hline BPP & Brake Pedal Position \\
\hline CAFE & Corporate Average Fuel Economy \\
\hline CAN & Controller Area Network \\
\hline $\mathrm{CD}$ & Charge Depleting \\
\hline CHGA_AUX & charger auxiliary voltage signal \\
\hline CHGA_WK & charger wake signal \\
\hline $\mathrm{CI}$ & Compression Ignition \\
\hline $\mathrm{CO}$ & Carbon Monoxide \\
\hline $\mathrm{CO}_{2}$ & Carbon Dioxide \\
\hline CS & Charge Sustaining \\
\hline $\mathrm{CVT}$ & Continuously Variable Transmission \\
\hline $\mathrm{DBC}$ & Damping Bypass Clutch \\
\hline $\mathrm{DC}$ & Direct Current \\
\hline DCA & Dynamic Consumer Acceptance \\
\hline DECM & Diesel Engine Control Module \\
\hline DFMEA & Design Fault Mode and Effect Analysis \\
\hline DOE & Department of Energy \\
\hline $\mathrm{DPF}$ & Diesel Particulate Filter \\
\hline EDS & Electric Distribution System \\
\hline EGR & Exhaust Gas Recirculation \\
\hline EM & Electric Motor \\
\hline EPA & Environmental Protection Agency \\
\hline
\end{tabular}




\begin{tabular}{|c|c|}
\hline EPO & Emergency Power Off \\
\hline E-REV & Extended-Range Electric Vehicle \\
\hline ESS & Energy Storage System \\
\hline $\mathrm{EV}$ & Electric Vehicle \\
\hline EVT & Electrically Variable Transmission \\
\hline FBD & Free Body Diagram \\
\hline FE & Fuel Economy \\
\hline FTA & Fault Tree Analysis \\
\hline FWD & Front Wheel Drive \\
\hline GHG & Greenhouse Gas \\
\hline GM & General Motors \\
\hline GMLAN & GM High Speed CAN \\
\hline GPS & Global Positioning System \\
\hline GREET & Greenhouse Gas, Regulated Emissions, and Energy Use in Transportation Model \\
\hline $\mathrm{HC}$ & Hydrocarbon \\
\hline HCP & Hybrid Control Processor \\
\hline $\mathrm{HEV}$ & Hybrid Electric Vehicle \\
\hline HIL & Hardware-In-the-Loop \\
\hline $\mathrm{HV}$ & High Voltage \\
\hline HVIL & High Voltage Inter-Lock \\
\hline HWFET & Highway Fuel Economy Driving Schedule \\
\hline IC & Internal Combustion \\
\hline ICE & Internal Combustion Engine \\
\hline ID & Identification \\
\hline IEEE & Institute of Electrical and Electronics Engineers \\
\hline IGN & Ignition \\
\hline LSO & Low Side Out \\
\hline LUT & Lookup Table \\
\hline MCPA & Motor Control Processor A \\
\hline MCPB & Motor Control Processor B \\
\hline MHV & Mild Hybrid Vehicle \\
\hline MIMO & Multiple Input and Multiple Output \\
\hline MPG & Mile per Gallon \\
\hline MPGge & Mile per Gallon gasoline equivalent \\
\hline MUDS & Morgantown Urban Drive Schedule \\
\hline MVM & Mean Value Model \\
\hline NHTSA & National Highway Traffic Safety Administration \\
\hline NOx & Oxides of Nitrogen \\
\hline NREL & National Renewable Energy Laboratory \\
\hline $\mathrm{OCV}$ & Open Circuit Voltage \\
\hline ODE & Ordinary Differential Equation \\
\hline
\end{tabular}




\begin{tabular}{|c|c|}
\hline OEM & Original Equipment Manufacturer \\
\hline ORSE & On Road Safety Evaluation \\
\hline PDE & Partial Differential Equations \\
\hline PEU & Petroleum Energy Use \\
\hline PHEV & Plug-in Hybrid Electric Vehicle \\
\hline PI & Proportional-Integral \\
\hline PM & Particulate Matter \\
\hline PMSM & Permanent Magnet Synchronous Motor \\
\hline PSAT & Power System Analysis Toolbox \\
\hline PTEB & Powertrain Extension Bus \\
\hline PWM & Pulse Width Modulation \\
\hline PWR & Power Line \\
\hline R19HW & Route 19 Highway \\
\hline RTA & Rear Traction Assistance \\
\hline RWD & Rear Wheel Drive \\
\hline SAE & Society of Automotive Engineers \\
\hline SCR & Selective Catalytic Reduction \\
\hline SCU & Supervisory Control Unit \\
\hline SFTP & US06 Supplemental Federal Test Procedure \\
\hline SI & Spark Ignition \\
\hline SIL & Software-In-the-Loop \\
\hline SOC & State of Charge \\
\hline SoftACU & Software Simulation of the ACU \\
\hline SoftBMS & Software Simulation of the BMS \\
\hline SUV & Sport Utility Vehicle \\
\hline TCM & Transmission Control Module \\
\hline THS & Toyota Hybrid System \\
\hline THS-II & Toyota Hybrid System II \\
\hline TPIM & Transmission Powertrain Inverter Module \\
\hline TTW & Tank-To-Wheel \\
\hline UDDS & Urban Dynamometer Driving Schedule \\
\hline UF & Utility Factor \\
\hline US & United States \\
\hline Veh_WAKE & Vehicle Wake Signal \\
\hline vs. & versus \\
\hline VTS & Vehicle Technical Specification \\
\hline VVT & Variable Valve Timing \\
\hline WTP & Well-To-Pump \\
\hline WTW & Well-To-Wheel \\
\hline WVLAN & West Virginia High Speed CAN \\
\hline WVU & West Virginia University \\
\hline
\end{tabular}




\section{CONTENTS}

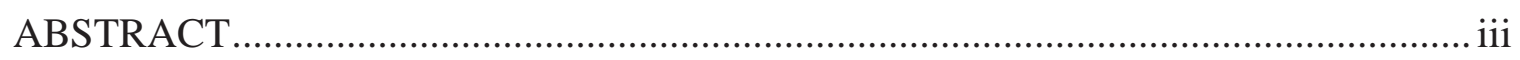

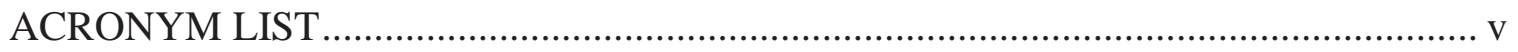

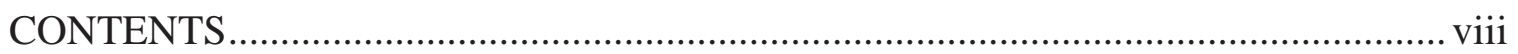

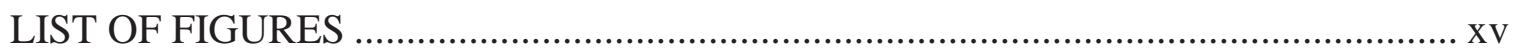

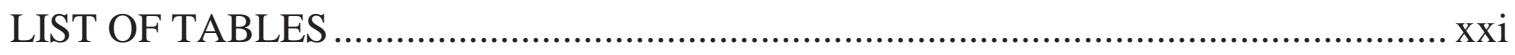

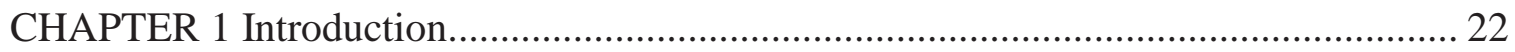

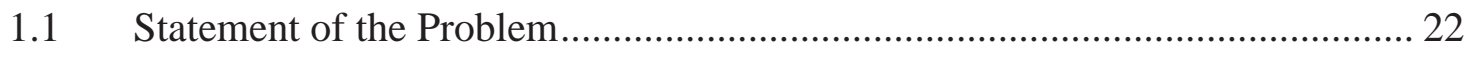

1.1.1 Petroleum Oil Usage in Transportation ................................................ 22

1.1.2 Automotive Tailpipe Emissions......................................................... 25

1.2 Objective of the Research ........................................................................ 26



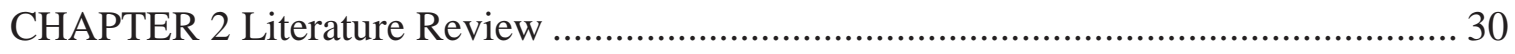

$2.1 \quad$ Hybrid Electric Vehicle Technologies ...................................................... 30

$2.2 \quad$ Hybrid Electric Vehicle Introduction ...................................................... 33

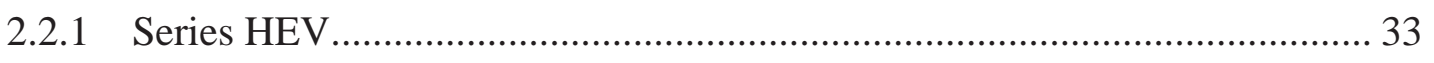

2.2.2 Parallel HEV ................................................................................... 35

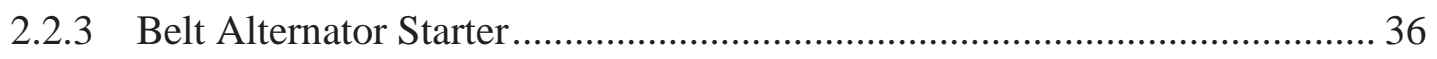

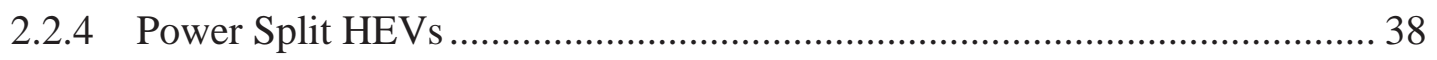

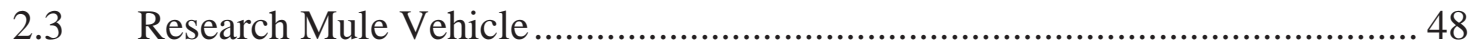

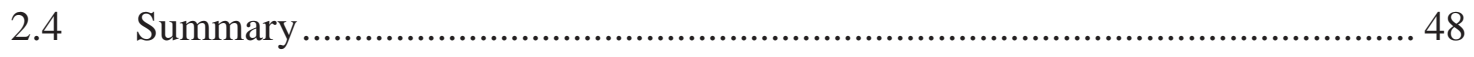


CHAPTER 3 Vehicle Architecture Selection Process................................................... 50

3.1 Vehicle Architecture Consideration............................................................ 50

3.1.1 Vehicle Technical Specifications............................................................. 50

3.2 Hybrid Electric Vehicle Architecture Selection Procedure ........................... 60

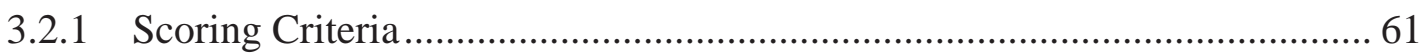

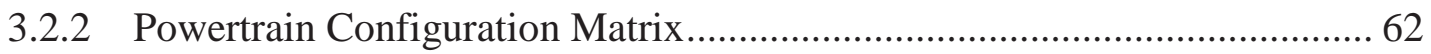

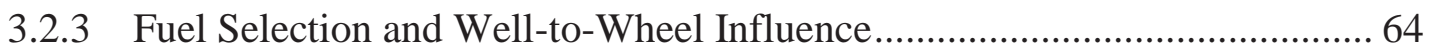

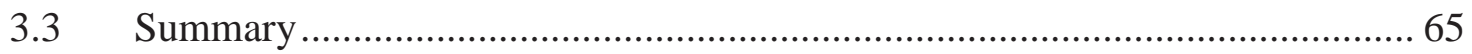

CHAPTER 4 2-Mode Hybrid Electric Vehicle Model ................................................. 68

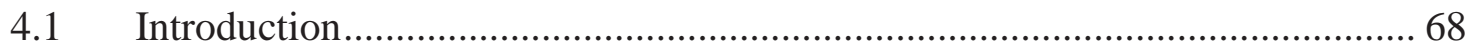

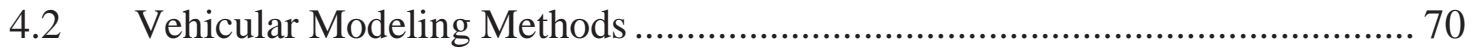

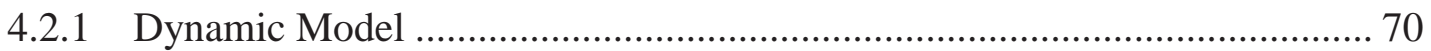

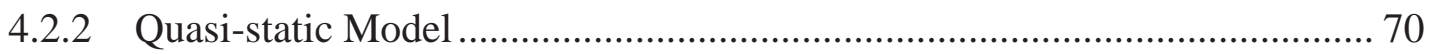

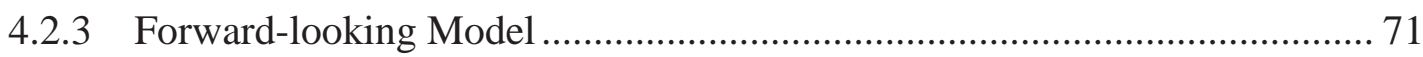

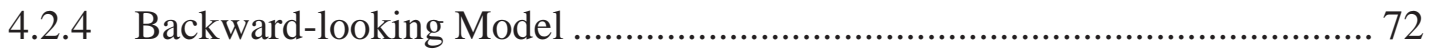

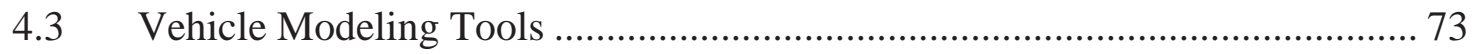

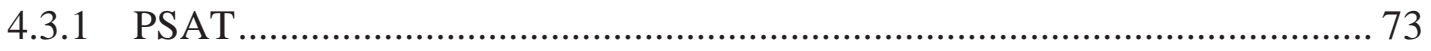

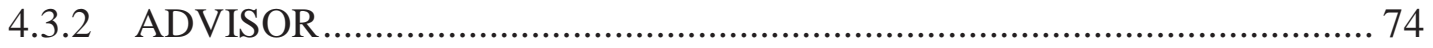

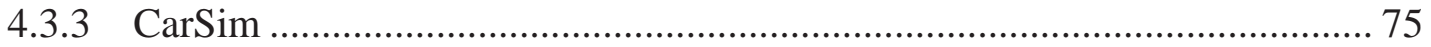

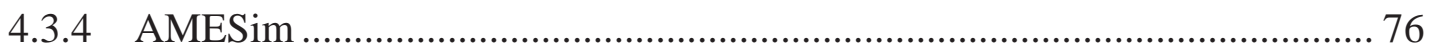

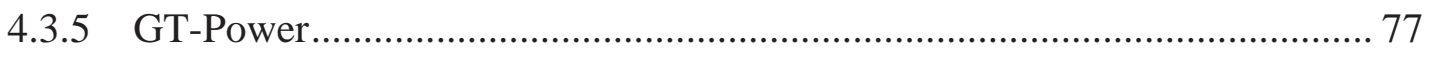

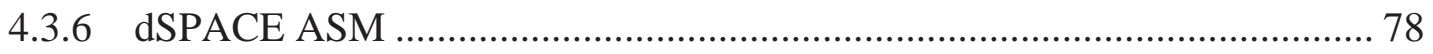

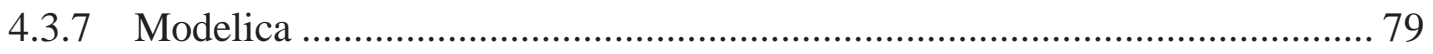

$4.4 \quad$ 2-Mode Hybrid Electric Vehicle Modeling ................................................ 80

4.4.1 2-Mode Hybrid Powertrain Schematics................................................... 80 


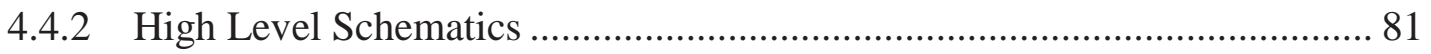

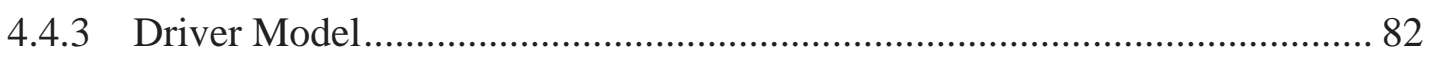

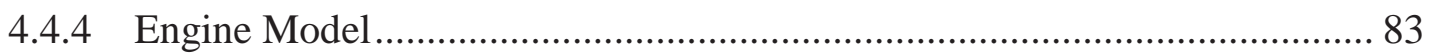

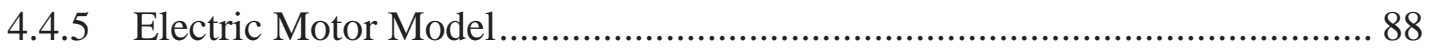

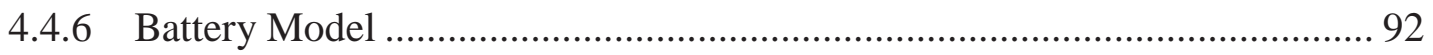

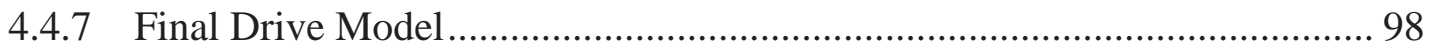

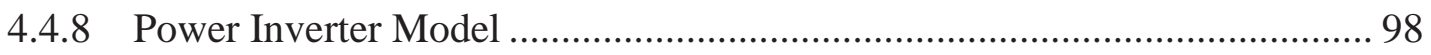

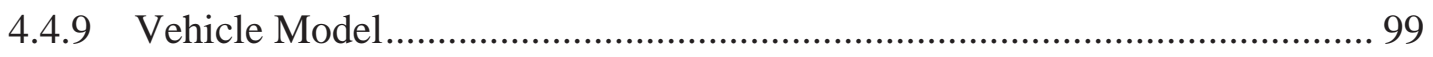

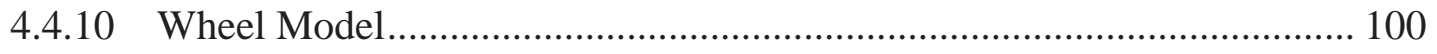

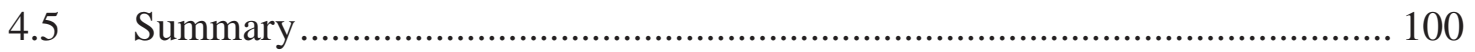

CHAPTER 5 2-Mode Hybrid Transmission Model .................................................... 102

5.1 Quasi-static Model of Single Planetary Gear ........................................... 102

5.2 Model of HEV with Single Planetary Gear Set ........................................ 104

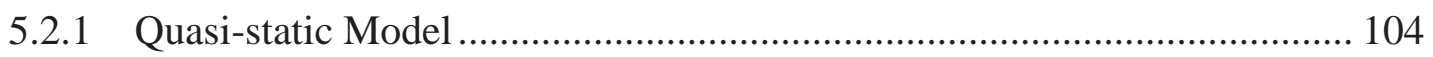

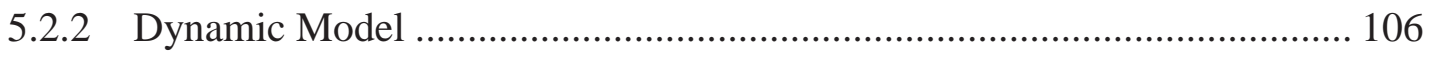

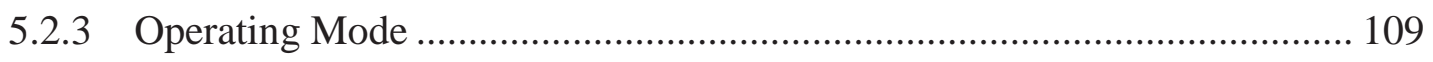

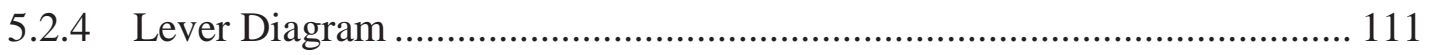

5.3 2-Mode HEV Model ............................................................................... 113

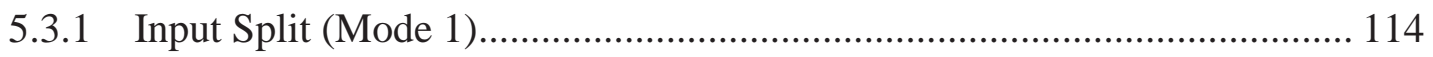

5.3.2 Compound Split (Mode 2) ................................................................. 117

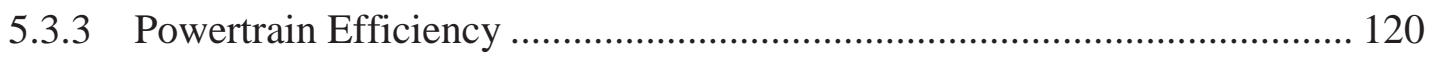



CHAPTER 6 2-Mode Hybrid Powertrain Control Algorithm ...................................... 125

6.1 Objective of the Hybrid System Control Strategy ....................................... 125

6.2 Hybrid Vehicle Modes of Operation ........................................................ 126 
6.2.1 Electric Launch ........................................................................... 126

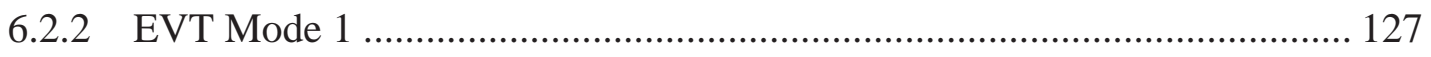

6.2.3 EVT Mode 2 _................................................................................. 127

6.2.4 Regenerative Braking................................................................... 127

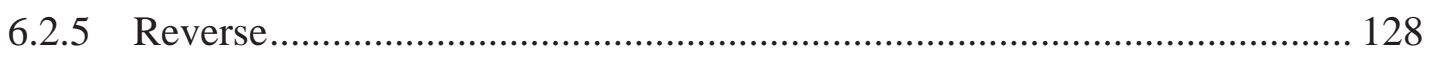

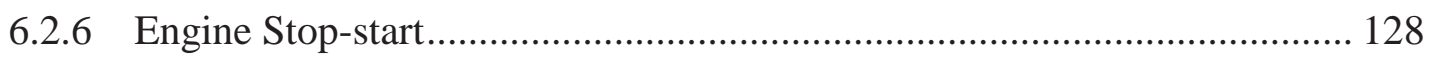

6.3 Overall Hybrid Control System Architecture ........................................... 128

6.4 2-Mode HEV Control Software Interface................................................. 130

6.5 Hybrid Powertrain System Control Strategy ............................................ 132

6.5.1 Driver Operating Mode................................................................... 133



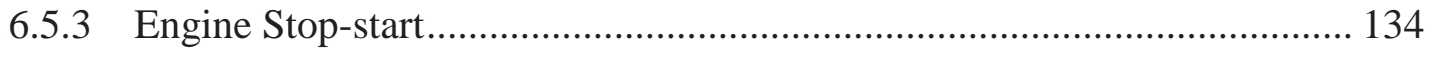

6.5.4 Transmission Shifting Algorithm ..................................................... 136

6.5.5 High Voltage Battery State of Charge Sustaining Algorithm.................... 138

6.5.6 Regenerative Braking Strategy ......................................................... 141

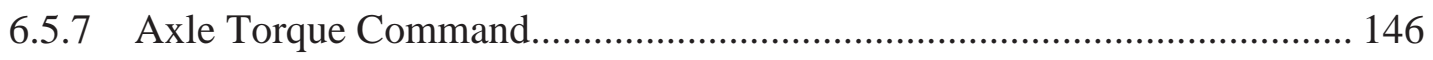

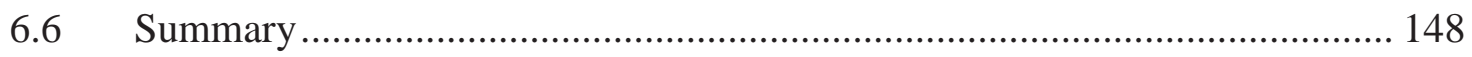

CHAPTER 7 Simulation and Development of Auxiliary Control Unit and Battery Management System ..................................................................................... 149

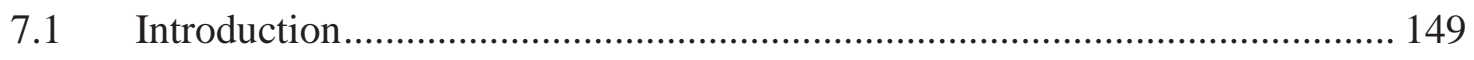

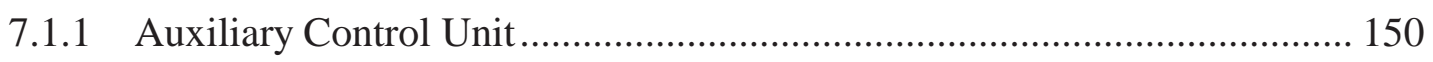

7.1.2 Battery Management System ............................................................... 152

7.2 ACU Control Algorithm Development.................................................... 155

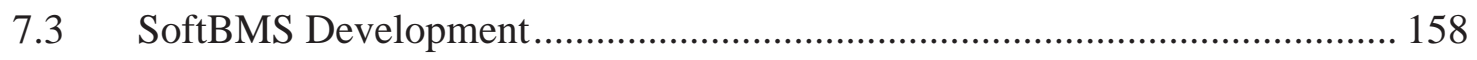

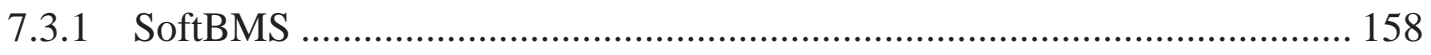

7.3.2 Electric Distribution System Plant Model .............................................. 164 


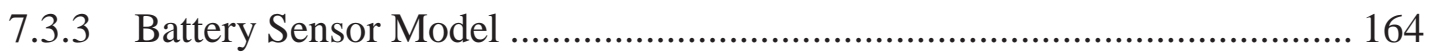

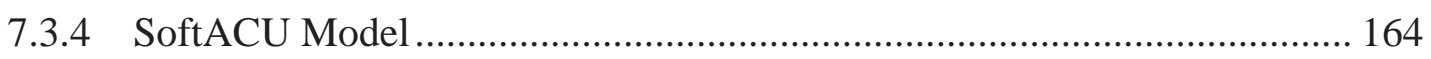

7.3.5 High Voltage Battery Model.......................................................................... 166

7.4 Test Results and Summarization...................................................................... 166

7.4.1 Test SoftACU Control Commands .............................................................. 166

7.4.2 Test of the SoftACU to Close/Open the Main Contactors............................. 168

7.4.3 Test of the ACU Response to Sensor Failures............................................... 169

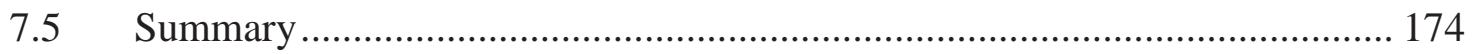

CHAPTER 8 2-Mode Hybrid Control System Implementation........................................ 175

8.1 Powertrain Integration Introduction............................................................. 175

8.1.1 Engine Bay Integration ............................................................................. 175

8.1.2 High Voltage Battery Integration................................................................... 176

8.2 Distributed Hybrid Vehicle Control System.................................................... 177

8.2.1 Supervisory Control Unit ......................................................................... 179

8.2.2 Auxiliary Control Unit ............................................................................ 180

8.2.3 Transmission Power Inverter Module..................................................... 180

8.2.4 Transmission Control Module .................................................................. 180

8.2.5 Diesel Engine Control Module ……………………………......................... 181

8.2.6 Battery Management System ........................................................................ 181

8.3 Hybrid Powertrain Control Integration ............................................................. 181

8.3.1 Real-time System Controller Introduction.................................................... 181

8.3.2 Accelerator Pedal Integration ....................................................................... 181

8.3.3 Engine Ignition and Stop-start Control ....................................................... 184

8.3.4 Hybrid Fueling System ............................................................................... 185

8.3.5 High Voltage Battery Electric System .......................................................... 186 


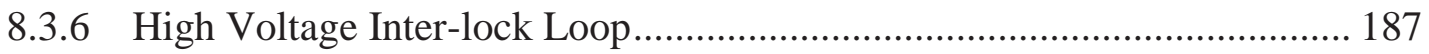

8.3.7 High Voltage Battery Management System ........................................... 189

8.3.8 Maximum Discharging Power and Current ............................................. 190

8.3.9 Maximum Charging Power and Current............................................... 192

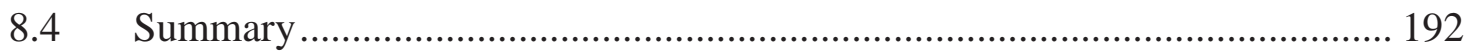

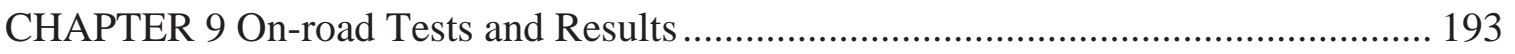

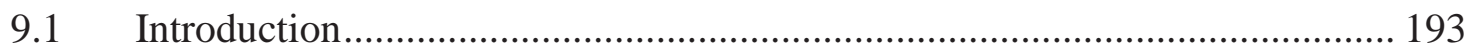

9.2 Control System Calibration ................................................................... 193

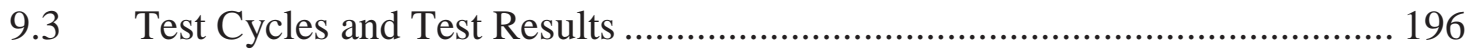

9.3.1 On Road Safety Evaluation Test.......................................................... 196



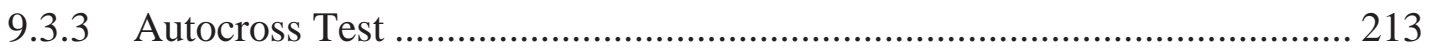

9.3.4 Acceleration Test ................................................................................. 213



9.3.6 Combined City \& Highway Test 1 ..................................................... 217

9.3.7 Combined City \& Highway Test 2 ...................................................... 219

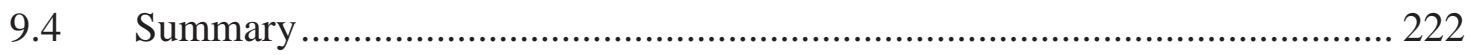

CHAPTER 10 Conclusions and Recommendations .................................................. 224

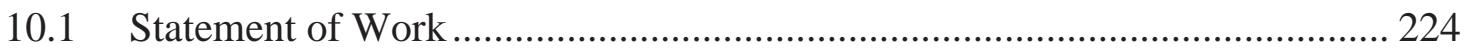

10.1.1 Hybrid Electric Vehicle Simulation ................................................... 225

10.1.2 Hybrid Powertrain Architecture Selection ............................................ 226

10.1.3 2-Mode Hybrid Control Algorithm Development .................................. 227

10.1.4 Hybrid Control System Implementation .............................................. 230

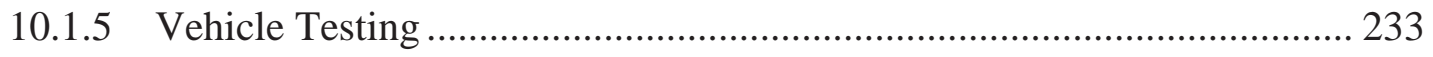

10.2 Contributions and Conclusions ........................................................... 234 
10.3 Future Work and Recommendations ..................................................... 235

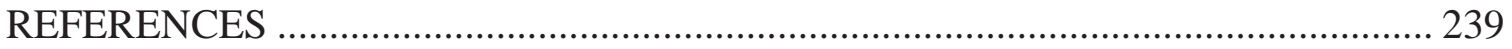




\section{LIST OF FIGURES}

Figure 1-1: United States petroleum production and consumption, 1973-2035 [5]....... 24

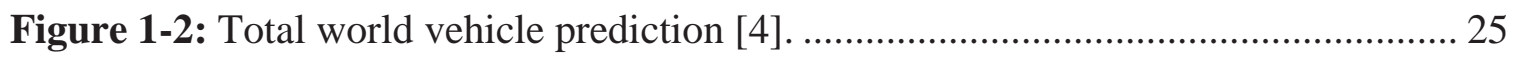

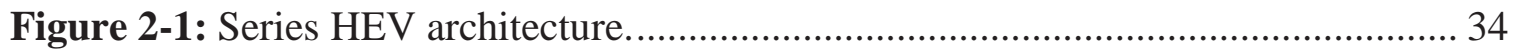

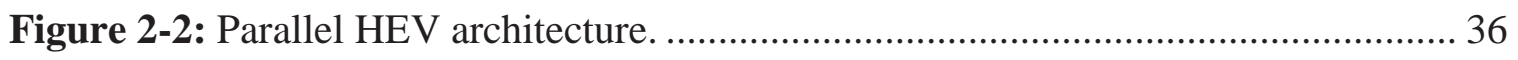

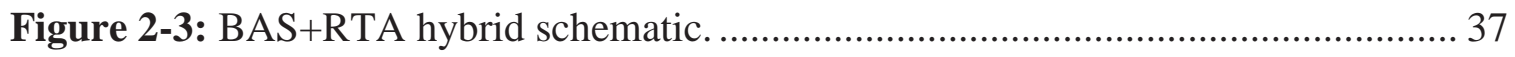

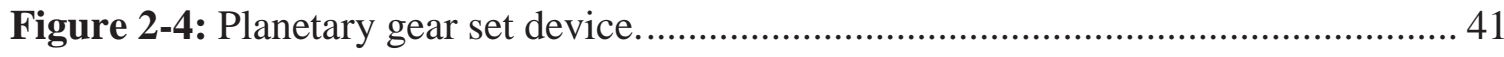

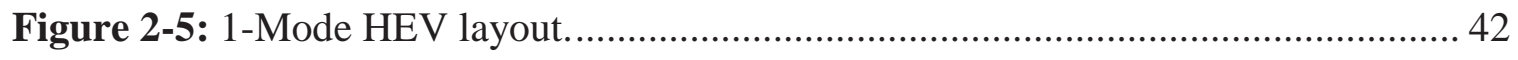

Figure 2-6: 2-Mode transmission diagram [19].................................................... 44

Figure 2-7: Power flow through mechanical and electrical paths [20]........................ 45

Figure 2-8: 2-Mode EVT driving to limit electrical power [20]................................. 45

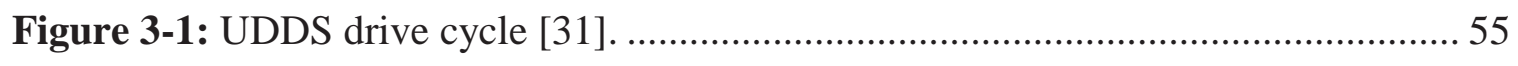

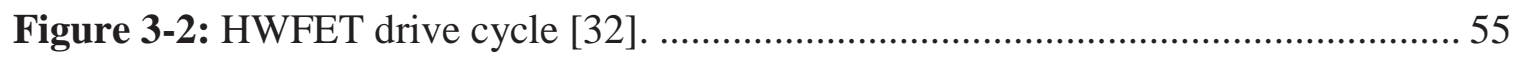

Figure 3-3: The normative utility factor. .......................................................... 57

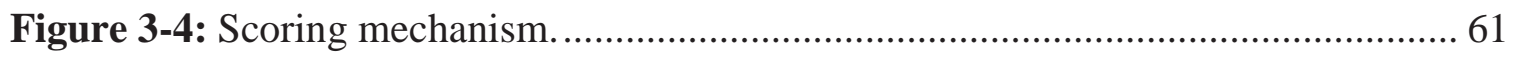

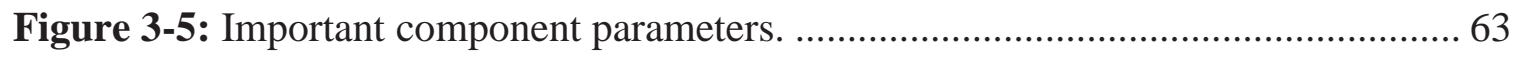

Figure 3-6: 2-Mode fuel economy, PEU and GHG emissions comparison..................... 65

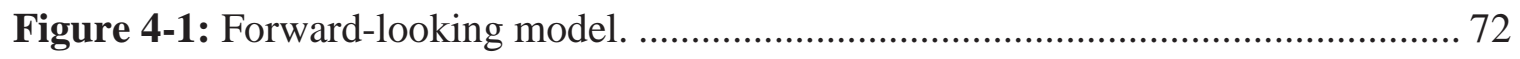

Figure 4-2: Backwards-looking series hybrid model............................................. 73

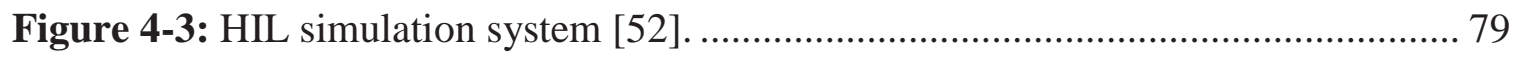

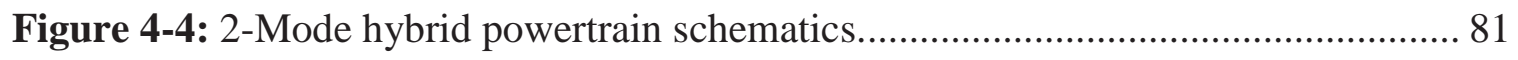

Figure 4-5: 2-Mode hybrid electric vehicle model architecture. ................................. 82 


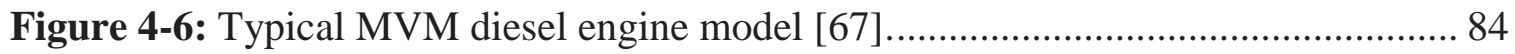

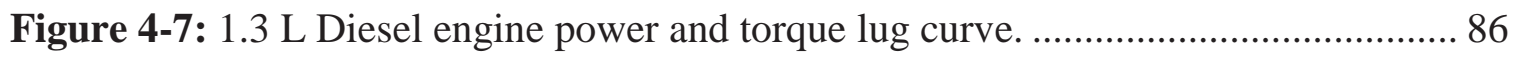

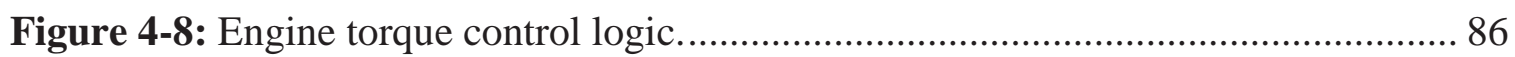

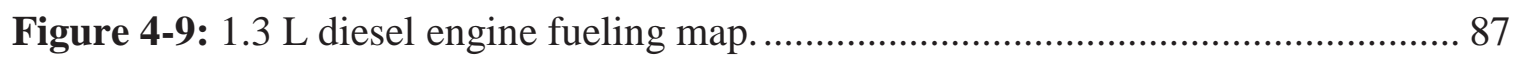

Figure 4-10: $1.3 \mathrm{~L}$ diesel engine fueling rate calculation control logic........................ 88

Figure 4-11: 2-Mode electric motor continuous torque and 10-second maximum



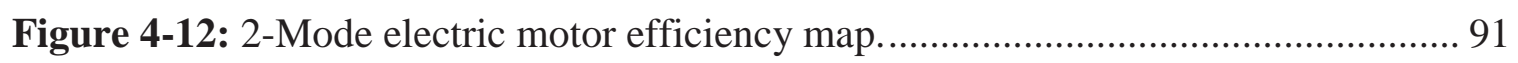



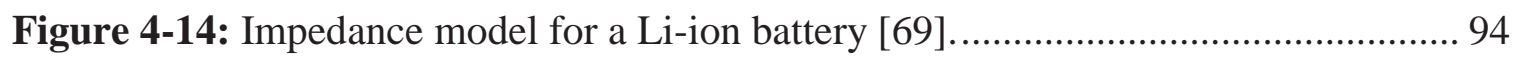

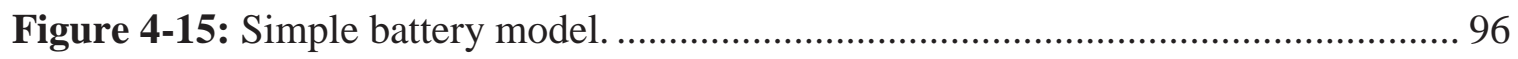

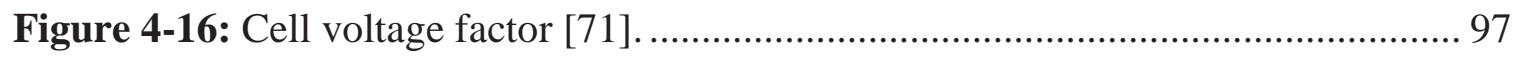

Figure 4-17: Battery cell voltage as a function of SOC and current [71]..................... 97

Figure 4-18: DC/DC power converter model. ....................................................... 99

Figure 5-1: Single planetary gear set diagram................................................... 103



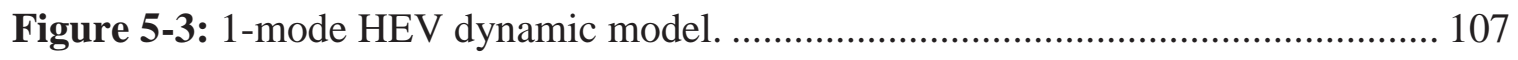

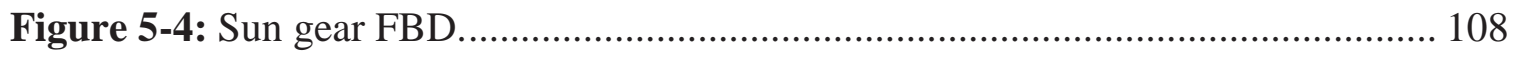

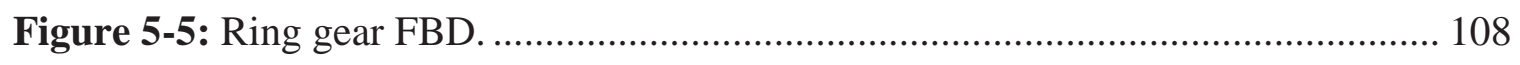

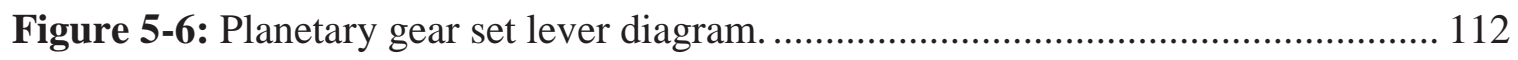

Figure 5-7: Planetary gear set lever diagram speed triangle..................................... 112

Figure 5-8: 1-mode HEV powertrain components speed limits................................ 113

Figure 5-9: 2-Mode HEV configuration with planetary gear sets. .............................. 114

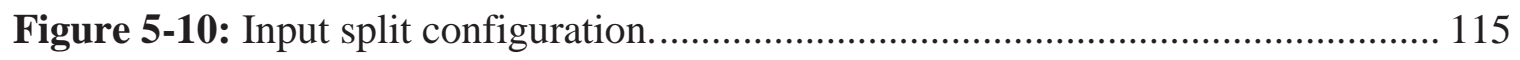

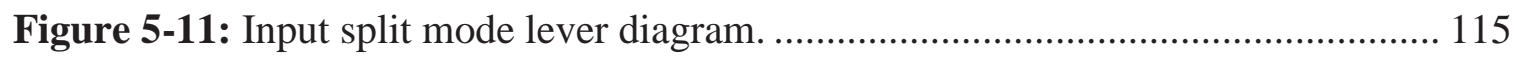

Figure 5-12: Vehicle operation in Mode 1 with engine off and engine on................... 116 


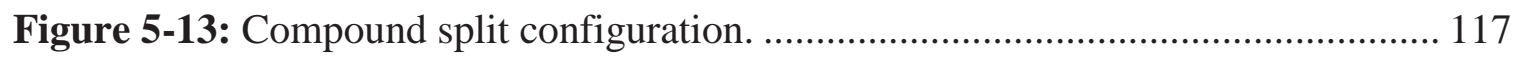

Figure 5-14: Compound split mode lever diagram.................................................. 118

Figure 5-15: Compound split mode single lever diagram. ......................................... 118

Figure 5-16: Driving scenarios in compound split mode. ........................................ 119

Figure 5-17: 2-Mode powertrain efficiency in Mode 1........................................... 121

Figure 5-18: 2-Mode powertrain efficiency with 2 EVT modes. ................................ 122

Figure 5-19: Overall powertrain efficiency using 1-Mode and 2-Mode. ...................... 123

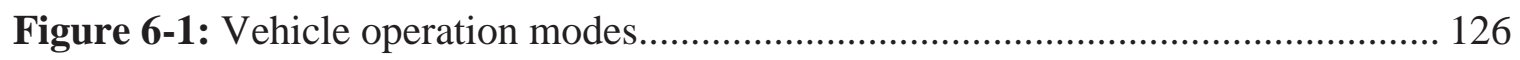

Figure 6-2: Overall hybrid control system diagram............................................. 129

Figure 6-3: 2-Mode HEV powertrain control methodology..................................... 131

Figure 6-4: 2-Mode HEV propulsion control strategy............................................ 133

Figure 6-5: Engine desired status control algorithm............................................. 136

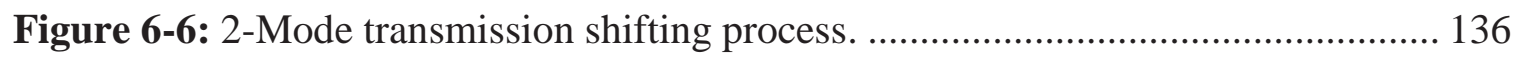

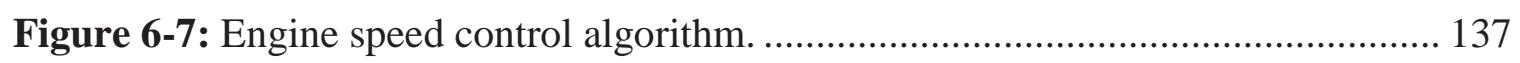

Figure 6-8: SOC sustaining control algorithm diagram...................................... 138

Figure 6-9: High voltage battery PI controller....................................................... 139

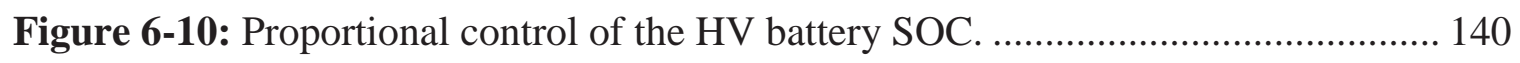

Figure 6-11: Deceleration addition by regenerative braking. ................................... 142

Figure 6-12: Regenerative braking power capture. ................................................ 143

Figure 6-13: Constant maximum charging current................................................ 144

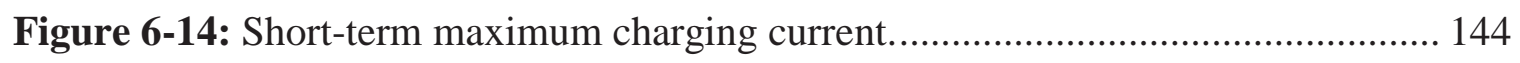

Figure 6-15: Electric motor constant maximum generating torque and short term

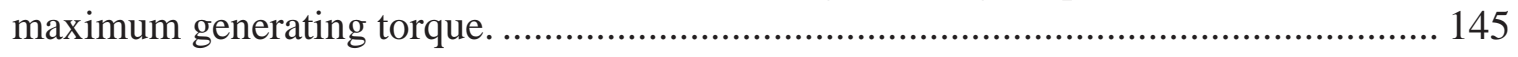

Figure 6-16: Regenerative braking control algorithm. ......................................... 146

Figure 6-17: Maximum axle torque command. .................................................. 147

Figure 6-18: Axle torque command with different APP........................................ 148 
Figure 7-1: Model integration of high voltage system. .......................................... 150

Figure 7-2: ACU interface in the 2-Mode HEV . ................................................... 151

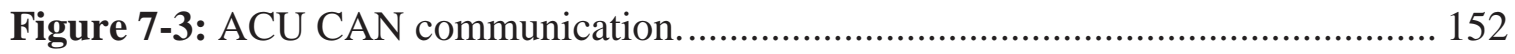

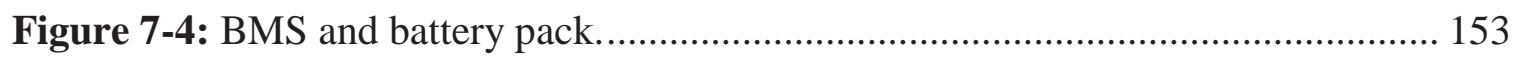

Figure 7-5: High voltage battery main contacts control algorithm............................ 158

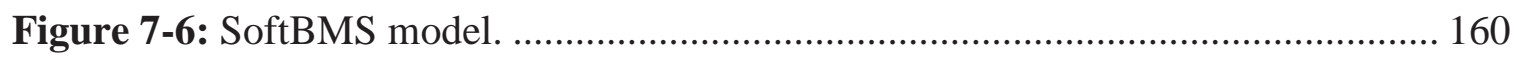

Figure 7-7: High voltage battery constraints model. .............................................. 162

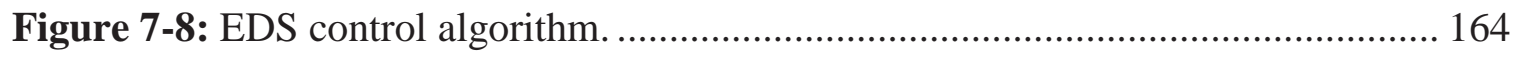



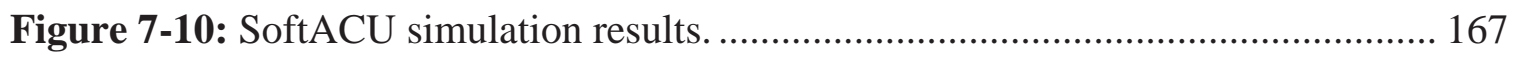

Figure 7-11: In-vehicle test of ACU control results. ............................................ 168

Figure 7-12: Test of SoftBMS open and close main contactor program...................... 169

Figure 7-13: Ground fault detected before main contactor closed. ............................ 170

Figure 7-14: Ground fault detected after main contactor closed. .............................. 171

Figure 7-15: HVIL failure before main contactor closed. ...................................... 172

Figure 7-16: HVIL failure after main contactor closed. ......................................... 173

Figure 7-17: EPO failure after main contactor closed........................................... 174

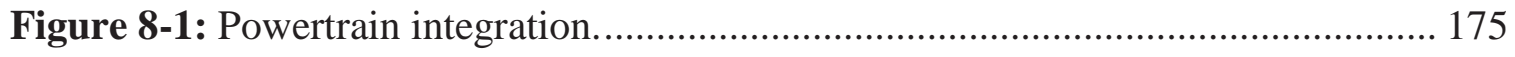

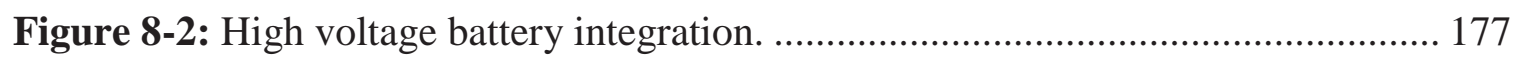

Figure 8-3: Distributed hybrid vehicle control system. ......................................... 178

Figure 8-4: 2-Mode key powertrain control CAN signals........................................ 179

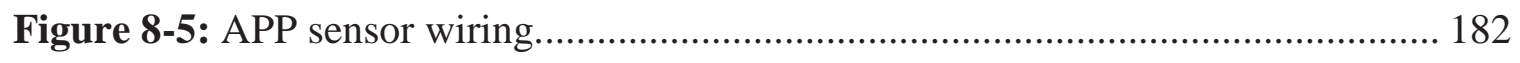

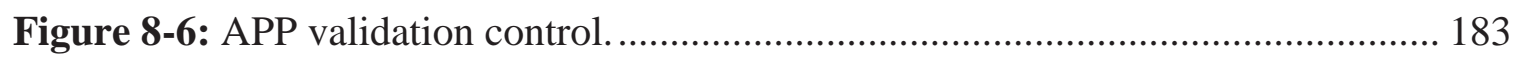

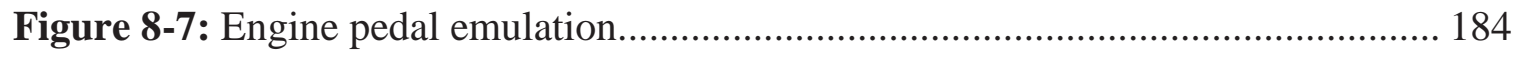

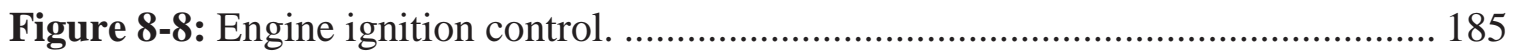




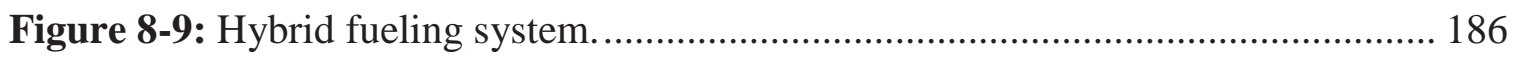

Figure 8-10: High voltage battery electrical system............................................ 187

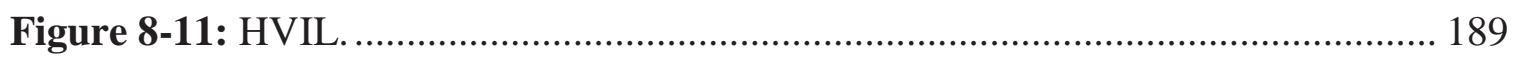

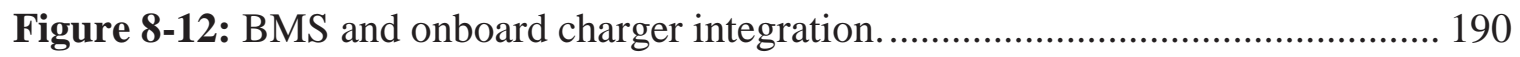

Figure 8-13: Steady-state maximum discharging current....................................... 191

Figure 8-14: High voltage battery maximum discharging power algorithm. ............... 192

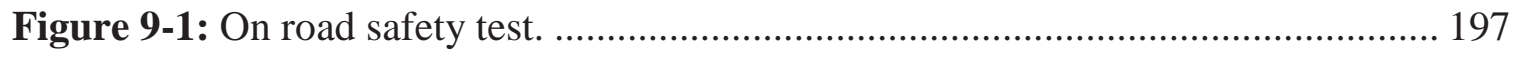

Figure 9-2: ORSE test components temperature diagram. ...................................... 197

Figure 9-3: ORSE test engine operating vs. time. ................................................ 198

Figure 9-4: ORSE test engine operating vs. engine efficiency................................. 198

Figure 9-5: ORSE test high voltage battery operation............................................ 199

Figure 9-6: Morgantown urban drive schedule (MUDS)......................................... 200

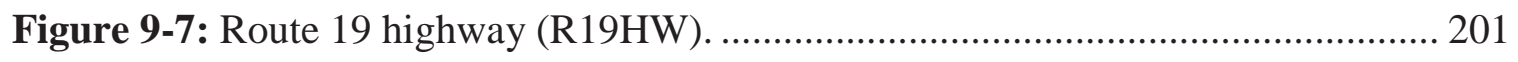

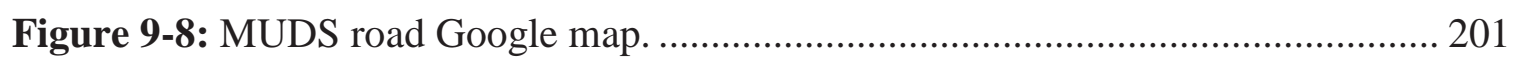

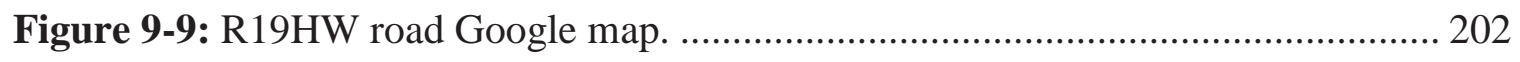

Figure 9-10: Engine stop-start status over the MUDS cycle.................................... 205

Figure 9-11: Engine operating points over the MUDS cycle. ................................... 206

Figure 9-12: Engine working points on the R19HW cycle. ..................................... 207

Figure 9-13: Transmission shifting in MUDS cycle............................................ 208

Figure 9-14: Transmission shifting in R19HW cycle............................................ 208

Figure 9-15: High voltage battery SOC sustaining in MUDS cycle............................ 209

Figure 9-16: High voltage battery SOC sustaining in R19HW cycle.......................... 209

Figure 9-17: M/G1 operation in MUDS cycle........................................................ 210

Figure 9-18: M/G2 operation in MUDS cycle.................................................... 211

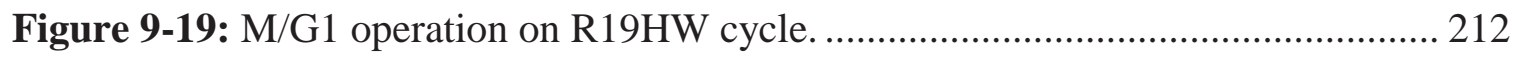


Figure 9-20: M/G2 operation on R19HW cycle. ................................................... 213

Figure 9-21: Acceleration test results. .............................................................. 214

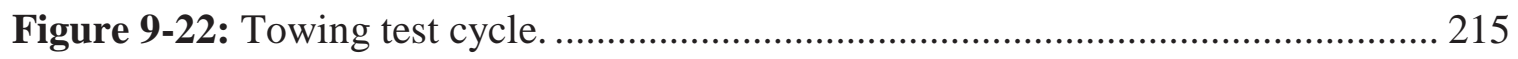

Figure 9-23: Towing test engine operation vs. time............................................. 215

Figure 9-24: Towing test engine operation vs. efficiency. ...................................... 216

Figure 9-25: Towing test high voltage battery operation. ...................................... 216

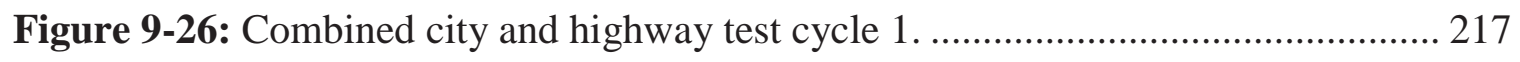

Figure 9-27: Combined City \& Highway Test 1 engine operation vs. time.................. 218

Figure 9-28: Combined City \& Highway Test 1 engine operation vs. engine

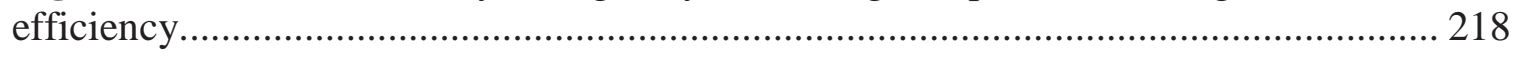

Figure 9-29: Combined City \& Highway Test 1 high voltage battery operation. ......... 219

Figure 9-30: Combined city and highway test cycle 2. ......................................... 220

Figure 9-31: Combined City \& Highway Test 2 engine operation vs. time................. 220

Figure 9-32: Combined City \& Highway Test 2 engine operation vs. engine

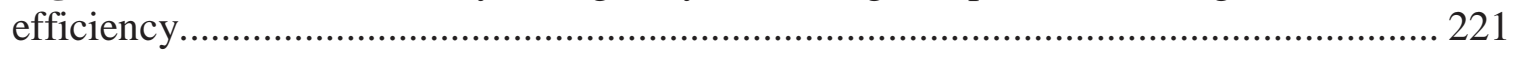

Figure 9-33: Combined City \& Highway Test 2 high voltage battery operation. ......... 222 


\section{LIST OF TABLES}

Table 1-1: Hybrid powertrain research procedure. ..................................................... 29

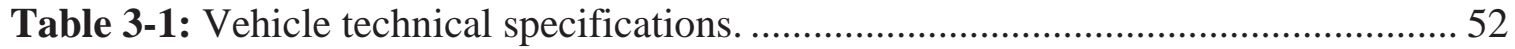

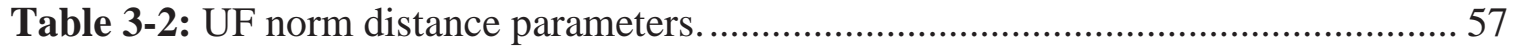

Table 3-3: Vehicle technical specifications and architecture evaluation criteria............. 61



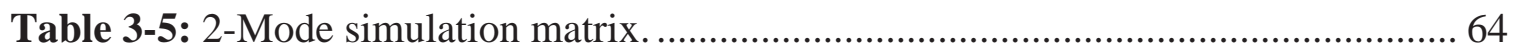

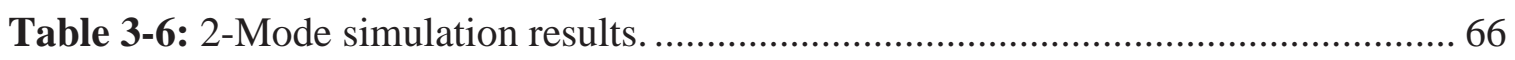



Table 3-8: 2-Mode hybrid electric crossover utility vehicle powertrain components

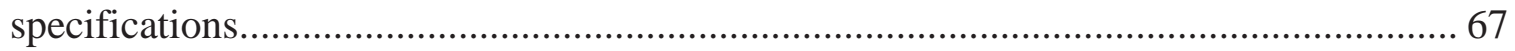

Table 5-1: THS powertrain configuration with planetary gear set. ............................. 105

Table 5-2: 1-mode HEV dynamic model parameters. ............................................. 107

Table 9-1: Hybrid control system calibration configurations. ................................... 196

Table 9-2: Fuel economy comparison of PSAT model and on-road test results. .......... 204



Table 10-1: Vehicle technical specification.....................Error! Bookmark not defined. 


\section{CHAPTER 1}

\section{INTRODUCTION}

\subsection{Statement of the Problem}

\subsection{1 $\quad$ Petroleum Oil Usage in Transportation}

Over the next 25 years, the energy demand for transportation is expected to increase more rapidly than in any other end-use sectors due to increasing demand for personal motor transportation as a result of rapidly developing global economies [1]. The limited petroleum reserves and increasing demand for oil seems not to be able to resolve in the short term. Maintaining energy sustainability is of interest to scientists and governments. Energy sustainability is not only a problem in one country or one region. It is a global challenge. Governments, scientists and engineers from all countries are joining together to find the solutions.

Petroleum is heavily consumed in automotive transportation. Automotive transportation consumes more than $71 \%$ of the world's total petroleum energy. The United States consumes nearly one quarter of the world oil production, over $70 \%$ percent of which is consumed in automotive transportation. Highway transportation accounts for $85 \%$ of the oil consumption for transportation in the U.S. and light-duty vehicles account for $66 \%$ of the oil consumption [2]. Over $95 \%$ of on-road vehicles in the world consume petroleum-based fuels [3]. 
Future oil demand in automotive transportation is going to be greater than ever. As shown in Figure 1-1, after two decades of effort to improve the efficiency of energy use in homes, factories and transportation, the energy use in homes is about the same, and energy use in industry has dropped. However, the energy use in transportation is going up, and the trend is not optimistic. During the past three decades, vehicle operating efficiency has been improved from 15.9 miles per gallon (MPG) in the US in 1980 to 22.6 MPG in 2008, with the advanced technologies applied in the vehicles. However, the increase in the number of vehicles on the road offsets all of the effort that advanced technologies have achieved to reduce fuel consumption. The number of the vehicles is soaring with the population explosion and flourishing economic development, shown in Figure 1-2 [4]. It is estimated that there are approximately one billion vehicles on the road in the world now. If the vehicle growth trend continues, the number of the vehicles on the earth will reach over two billion by 2030. This trend indicates the severe challenge from petroleum energy consumption for automotive transportation. Projections by the Energy Information Administration indicate that U.S. consumption of liquid fuels by the transportation sector will rise from 14.3 million barrels per day in 2011 to 16.7 million barrels per day in 2030. 


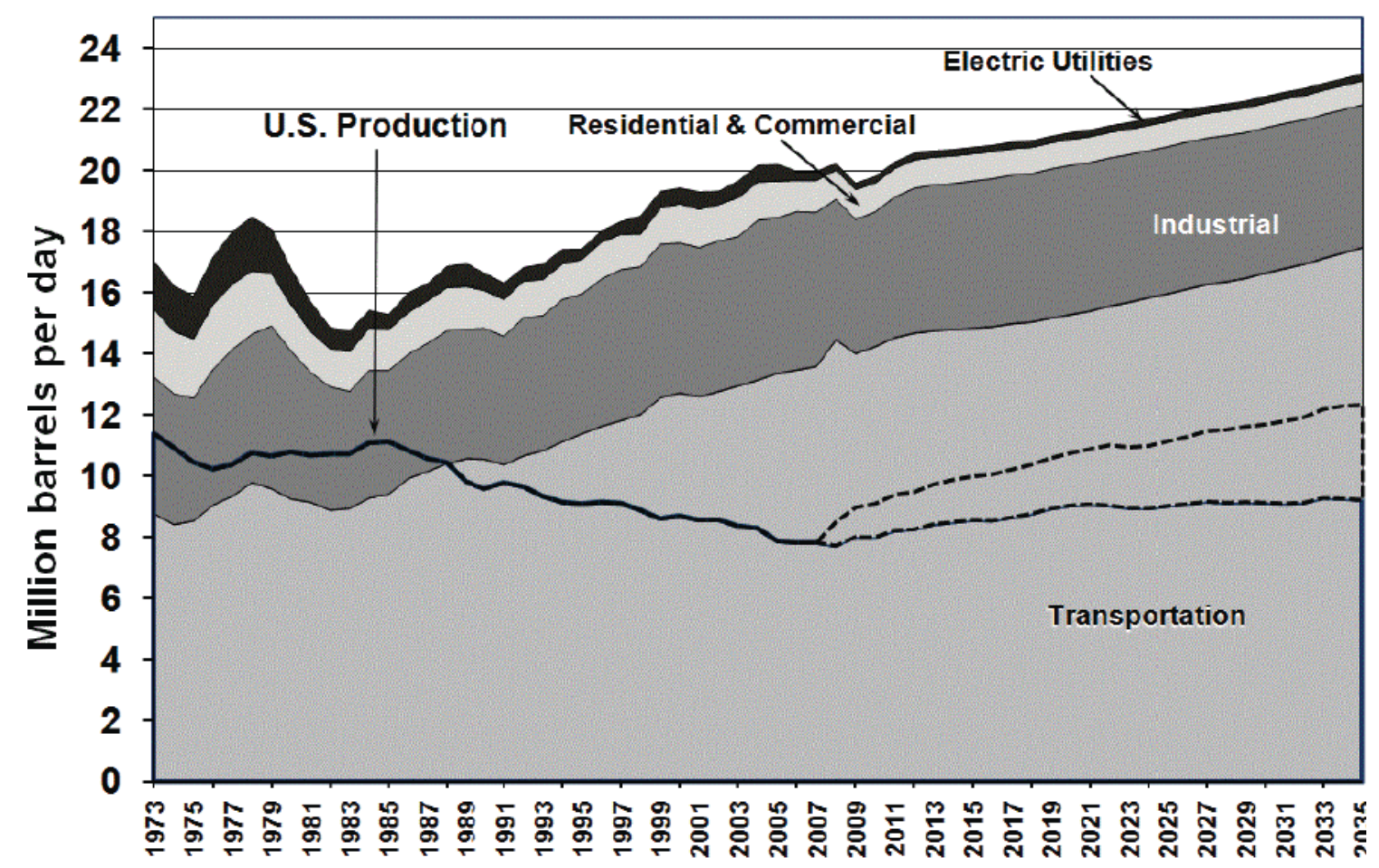

Figure 1-1: United States petroleum production and consumption, 1973-2035 [5].

The growth in the world's vehicle population is increasing the pressure on finite oil reserves. The world's remaining oil reserves are 1332.1 billion barrels, and $40 \%$ of the total world oil reserves has been consumed in the past 30 years [6]. If the consumption continues to increase and new technologies are not advanced to further increase the vehicle operating efficiency considerably, it will take less than 30 years to consume the rest of the proven conventional oil reservoir [6]. 


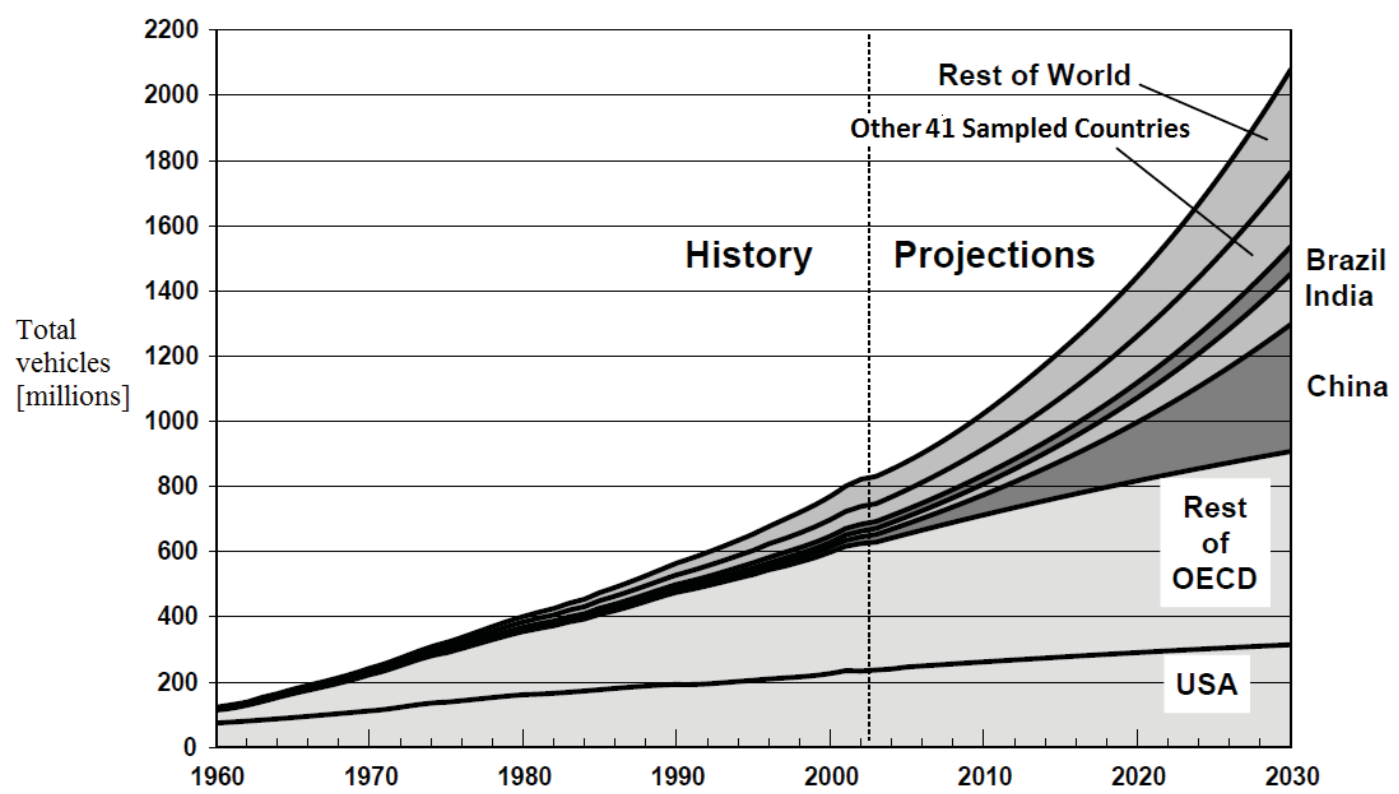

Figure 1-2: Total world vehicle prediction [4].

\subsection{2 $\quad \underline{\text { Automotive Tailpipe Emissions }}$}

Automotive transportation produces poisonous gases that not only do harm to human's health, but also have a negative impact on the environment, such as Green House Gas (GHG) emissions, global warming, and climatic changes. Fuel combustion in the Internal Combustion Engine (ICE) releases carbon dioxide $\left(\mathrm{CO}_{2}\right)$, unburned hydrocarbons (HC), carbon monoxide (CO), oxides of nitrogen $\left(\mathrm{NO}_{\mathrm{x}}\right)$, particulate matter (PM), and other GHGs. Other than carbon dioxide, vehicle tailpipe emissions have been reduced to a small fraction of the levels in the 1970s, by radical improvements in fuel combustion technologies and in aftertreatment technologies, like catalytic converters, diesel particulate filters and selective catalytic reduction (SCR). $\mathrm{CO}_{2}$ has never been regulated as a harmful vehicle pollutant. However, $\mathrm{CO}_{2}$ is a major contributor of GHGs. It is widely agreed that GHGs are associated with global warming, a major long-term threat to the environment. 
Burning one gallon of gasoline or diesel fuel in an Internal Combustion (IC) engine produces around $9 \mathrm{~kg} \mathrm{CO}$. Road transport delivers $16 \%$ of global man-made $\mathrm{CO}_{2}$ emissions (about13\% of total GHGs) [6]. In the United States, transportation's share of $\mathrm{CO}_{2}$ and NOx emissions from petroleum fuel are about $33 \%$ and $16 \%$ respectively. The gasoline share of transportation $\mathrm{CO}_{2}$ emissions is about 60\% [6]. Since $1990 \mathrm{CO}_{2}$ production from transportation has risen $46 \%$ and transportation has been the highest $\mathrm{CO}_{2}$ producing end-use sector since 1999. Projections place transportation related $\mathrm{CO}_{2}$ emissions over 2,500 million metric tons per year by 2030 .

To remove $\mathrm{CO}_{2}$ from the exhaust is extremely difficult and impractical for vehicles. There is no aftertreatment, like a catalytic converter that can be attached to a vehicle exhaust pipe to reduce $\mathrm{CO}_{2}$. The easiest way to reduce $\mathrm{CO}_{2}$ is to use less fuel or to use alternative fuels, either renewable or containing less or no carbon. Bio-fuels drawn from plant and animal fat provide an alternative as a renewable energy source. The $\mathrm{CO}_{2}$ generated by burning bio-fuels is absorbed by plants, same of which are used to produce bio-fuels. Theoretically, no extra $\mathrm{CO}_{2}$ is added into air in the bio-fuels process cycle. However, in real world there are processing costs in the form of $\mathrm{CO}_{2}$ to produce bio-fuels. $\mathrm{CO}_{2}$ capture and storage (CCS) to reduce $\mathrm{CO}_{2}$ is under research and is making some progress. This method requires a lot of effort and investment. There are currently four full-scale geologic $\mathrm{CO}_{2}$ storage projects in the world that have already successfully stored millions of tonnes of $\mathrm{CO}_{2}$ for many years without leakage [7].

\subsection{Objective of the Research}

Research on a novel 2-Mode hybrid powertrain was proposed to sustain petroleum energy usage and reduce the personal motor transportation dependence on 
petroleum fuel. The objective of this research was to develop a 2-Mode hybrid control system to achieve designated vehicle technical specifications in the following ways:

- Evaluate the hybrid control algorithm in the simulation environment to confirm that the designed 2-Mode hybrid electric vehicle meets the vehicle technical specifications.

- Model the 2-Mode electrically variable transmission to support the development of the hybrid control algorithm.

- Implement the hybrid control algorithm in real-time controllers.

- Test the vehicle on road to evaluate vehicle's safety features, performance and fuel economy.

The focus was not only on reducing the fuel consumption but also maintaining or improving the vehicle performance. The design of the 2-Mode hybrid control algorithm involved proceeding through specific steps based on hybrid control system design and validation criterion.

\subsection{Research Methodology}

The research combined analytical research and empirical research. In order to develop the 2-Mode hybrid control system for better vehicle performance and fuel economy, it was essential to analyze and model the 2-Mode hybrid electric vehicle prior to the implementation.

The analytical and simulation research were completed during the vehicle design phase. Through a literature review of the 2-Mode hybrid electric vehicle, the essential functions of the 2-Mode hybrid control system were identified: 
- 2-Mode electrically variable transmission shifting control in Electrically Variable Transmission (EVT) modes.

- Engine operation in high efficiency zones.

- Engine stop-start control.

- Regenerative braking control.

- High voltage battery State of Charge (SOC) charge sustaining control.

- Vehicle start, stop, reverse and drive control.

- Vehicle creep speed control.

- Hybrid system Controller Area Network (CAN) communication with stock electric control units.

- Key position recognition and driving mode recognition.

A rule-based hybrid control system has been proven to be an effective method for a complex system with multiple inputs and multiple outputs [8, 9]. A rule-based control method was applied to implement the 2-Mode hybrid control algorithm. A rule-based hybrid control algorithm was developed based on analytical and research of the 2-Mode hybrid electric vehicle.

The research included three phases: design, implementation and refinement, shown in Table 1-1. During the design phase, preliminary research was completed with a simulation model to evaluate the vehicle performance, fuel economy and GHGs emissions. In the implementation phase, the selected hybrid powertrain components were integrated in a mule vehicle. The rule-based hybrid control algorithm was implemented in two hybrid system controllers to achieve the desired functions. In the refinement phase, 
the prototype vehicle was calibrated to optimize the hybrid control function to maximize performance and fuel economy.

Table 1-1: Hybrid powertrain research procedure.

\begin{tabular}{|l|l|}
\hline Research Phases & Research Contents \\
\hline \multirow{5}{*}{ Design Phase } & 1. Defined problem to be solved \\
\cline { 2 - 2 } & 2. Preliminary research \\
\cline { 2 - 2 } & $\begin{array}{l}\text { a. Modeled hybrid vehicle powertrain in Powertrain } \\
\text { System Analysis Toolkit (PSAT) }\end{array}$ \\
\cline { 2 - 2 } & b. Selected the best hybrid powertrain \\
\cline { 2 - 2 } & C. Evaluated the hybrid powertrain specifications \\
\hline Implementation Phase & 1. Mechanical, electrical and control system design \\
\cline { 2 - 2 } & 2. Hybrid powertrain components bench test \\
\cline { 2 - 2 } & 3. Hybrid powertrain components integration and test \\
\hline & 1. Hybrid powertrain control algorithm refinement \\
\cline { 2 - 2 } & 2. Vehicle hybrid function test and refinement \\
\cline { 2 - 2 } & 3. Vehicle performance and fuel economy on-road test \\
\hline
\end{tabular}

Given below is a list of the tools used in developing the hybrid control system:

- MATLAB ${ }^{\circledR}$, Simulink \& Stateflow 2009

- VECTOR CANoe 7.1, CANape 8.0, CANcaseXL log

- dSPACE Mid-size Hardware-in-the-Loop (HIL) simulator

- General Motors (GM) on board diagnostics-II software

- Woodward MotoHawk \& MotoTune 2009 Software

- Microsoft Excel 2007 


\section{CHAPTER 2}

\section{LITERATURE REVIEW}

\subsection{Hybrid Electric Vehicle Technologies}

Advanced automobile technologies have been developed considerably to improve vehicle efficiency and minimize the impact to the environment. Huge resources are integrated annually to develop fuel-efficient vehicles that run on alternative fuels like biodiesel, ethanol, hydrogen, natural gas, battery, or with hybrid technology. Fuel efficiency and harmful emission cannot be solved with a single technology. Multiple technologies must be integrated together to meet the vehicular requirements for less fuel consumption, minimal environmental impact and better performance.

Hybrid electric vehicles (HEVs) provide an alternative to improve fuel economy, reduce tailpipe emissions and improve or maintain vehicle performance. An HEV is powered by a traditional IC engine and electric motor (EM) driven by high density batteries or capacitors. Conventional vehicles typically use only about $15 \%$ of the oil combustion energy to drive the car down the road and run useful accessories, and the rest of the energy is lost to engine and driveline inefficiencies, and idling [7]. Compared to conventional vehicles, an HEV is capable of improving fuel consumption significantly by increasing engine thermal efficiency by isolating the engine operation with the vehicle's road load, capturing regenerative braking energy and using battery power to drive the vehicle.

Huge efforts are bringing advanced technologies to HEVs to improve vehicle performance, reduce fuel consumption and tailpipe emission. Nowadays, an HEV can 
capture braking energy to charge the energy storage system, instead of letting the brakes waste energy by heat dissipation. Engine operation is optimized to run with the highest efficiency and to deliver peak power when needed. Battery technologies have made progress during the past decade to improve the power density, widen operating temperature range and reduce costs. Advanced control methods, like fuzzy logic, neural network, dynamic program and genetic algorithm, are used in hybrid powertrain control systems to improve vehicle operating efficiency and performance. A full hybrid electric vehicle equipped with powerful EM(s) can shut down the engine to operate like a Battery Electric Vehicle (BEV). Catalytic converters, diesel particulate filter and SCR with advanced control systems are being applied in HEVs to further reduce harmful tailpipe emissions.

Regenerative braking captures kinetic energy that an HEV can recycle and use to drive the vehicle later [10]. In a traditional vehicle braking system, the vehicle kinetic energy is converted to heat by friction in the brake linings and therefore wasted. In an $\mathrm{HEV}, \mathrm{EM}(\mathrm{s})$ and a battery are installed to drive the vehicle together with the IC engine. The EM is used as a generator during regenerative braking. The generator converts the mechanical power to electrical power to charge the Energy Storage System (ESS). Through regenerative braking, vehicle kinetic energy is recovered into electrical energy and stored in the batteries or capacitors.

Conventional friction brakes are still kept on hybrid vehicles for other functions that regenerative brake cannot replace, such as quicker stopping demands, anti-lock braking systems (ABS) and other safety features. For emergency stop demands, the conventional friction brakes are desired to effectively stop the car quickly. Regenerative 
braking effect drops off at low vehicle speed. Hence, conventional friction brakes are needed to stop the car completely. The regenerative braking effect is limited by the battery temperature and SOC, the EM dimensions and power electronics capability. The regenerative braking cannot be activated when vehicle is skidding or when the ABS is activating. Therefore, regenerative braking is not available at all times at the same level as friction brake, which limits its application in the vehicle.

The engine stop-start function allows an HEV to shut down the engine and cut the fuel supply during deceleration, standstill and low power driving conditions. Engine stopstart function in an HEV is different from engine stop-start function in a conventional vehicle. A conventional vehicle can achieve engine stop-start function by replacing the engine Original Equipment Manufacturer (OEM) alternator with a belt-driven starter/alternator [11]. An engine stop-start system is relatively simple, and it can be employed in both non-hybrid vehicles and hybrid vehicles. Ford announced that they will add stop-start technology to all of its conventional gasoline-powered cars. The test results [11] demonstrated a 5.3\% fuel consumption reduction in the city drive cycle due to traffic light stop, and approximately 4.0\% fuel consumption reduction in the highway drive cycle due to deceleration fuel cutoff. A conventional vehicle cannot shut off the engine when there is any power command to the axle, since the engine is the only power resource in a conventional vehicle. Engine stop-start function in an HEV can shut down the engine when the vehicle is driving under low load conditions. The engine stop-start function depends on the HEV architecture. If the electric powertrain in an $\mathrm{HEV}$ is strong enough to drive the vehicle independently, the HEV can operate stop-start to reduce fuel consumption. The more the HEV can shut down the engine during driving, the higher the 
fuel economy the vehicle can achieve. The Toyota Prius set the engine turn-on-power at 7 $\mathrm{kW}$ in the first generation and increased it to $11 \mathrm{~kW}$ in the second generation [12], which allowed more engine shutdown. The vehicle with engine stop-start function saves more fuel than a vehicle with an engine stop-start system since the vehicle stops fueling more frequently.

\subsection{Hybrid Electric Vehicle Introduction}

\subsection{1 $\quad$ Series HEV}

Series hybrid architectures decouple the engine operation from the vehicle speed bringing the IC engine (or fuel cell) to a nearly steady state operation. Therefore, the series architecture can be used to improve the amount of time during which the engine is run at optimal efficiency level. Although efficient in city driving environments, the series hybrid suffers in performance due to the inability of the IC engine to provide power directly to the road and the increased vehicle mass due to the added weight of the generator [13]. On the other hand, the series architecture is highly desirable in the case of an extended-range electric vehicle (E-REV) where the primary energy source is electricity and the range is obtained by a small IC engine which needs to provide only for the average tractive power (discounting the energy from regeneration). Figure 2-1 shows one typical series HEV architecture. The EM is placed between the IC engine and wheel, which decouples the IC engine operation from road conditions. This arrangement allows the IC engine to operate in its high efficiency area to charge the battery, which makes series hybrid appropriate to drive in urban areas. 

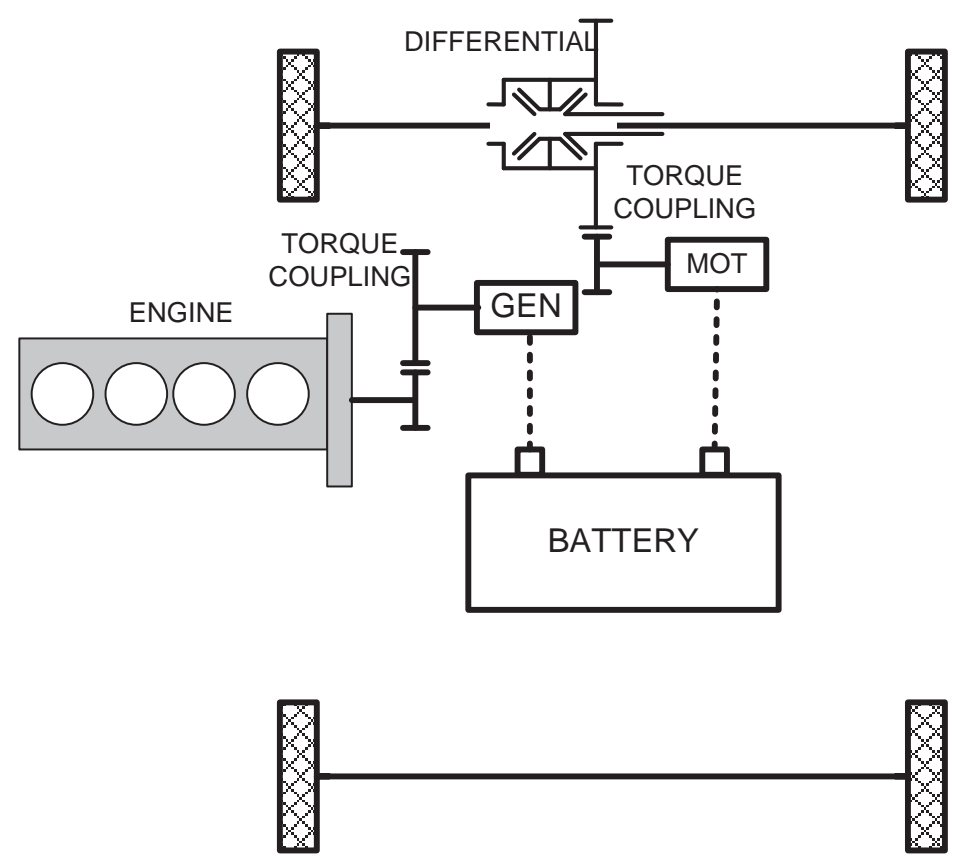

Figure 2-1: Series HEV architecture.

A series hybrid is more efficient when driven by the EM than by the IC engine. First, the EM can adjust the speed and torque in a wider range, which can eliminate the transmission from the powertrain. So the powertrain is lighter and less complex. Second, the EM can function the same way as the starter and the alternator, which decreases the weight and complexity even more. Furthermore, engine operation can be adjusted in a small high efficiency range. Therefore, the fuel economy can be improved and the engine control becomes easier.

Series HEVs also have some disadvantages. First, the addition of the high voltage battery, generator and likely the Direct Current (DC)/Alternative Current (AC) inverter for alternate current EM, increase the complexity and the weight of the powertrain. Second, engine power passing through an electric path, through generator and motor to drive the wheels, decreases powertrain efficiency. Third, the selection of driving motor is 
challenging for its size, mass and required electrical harnesses since it needs high voltage, high current and high torque to drive the vehicle alone. Moreover, safety concerns also arise due to the high voltage system in the HEV.

\subsection{2 $\quad \underline{\text { Parallel HEV }}$}

A parallel HEV is more like a conventional vehicle, which can be assisted by an extra electric motor. In fact, parallel HEV can be driven by EM, IC engine or both depending on the wheel load condition. A combined electric and combustion drivetrain as seen in the parallel architecture can theoretically produce efficiencies of up to 45\% [13]. The electric motor in a parallel architecture can be located pre-transmission, allowing an electric engine start, or post-transmission, allowing the option of electric only propulsions as well as maximizing the benefits of regenerative braking [14]. Figure 2-2 shows a typical parallel HEV architecture. The EM is placed in parallel with the IC engine to provide additional power to drive the vehicle. The EM can operate as a starter and an alternator, which can eliminate the traditional starter and alternator. Moreover, the EM can power the electrical accessories with higher efficiency, which may increases IC engine efficiency by removing the irregular parasitic load on the engine.

Parallel HEV can achieve the same performance as series HEV with a much smaller EM since the vehicle can be driven by the EM and IC engine. This downsizes the battery, EM and related electric parts, such as relays, wire gauge, and fuses. As a result, the cost added to the HEV is minimized. However, the control of the IC engine and EM to achieve optimal combined performance becomes a challenge for engineers. 


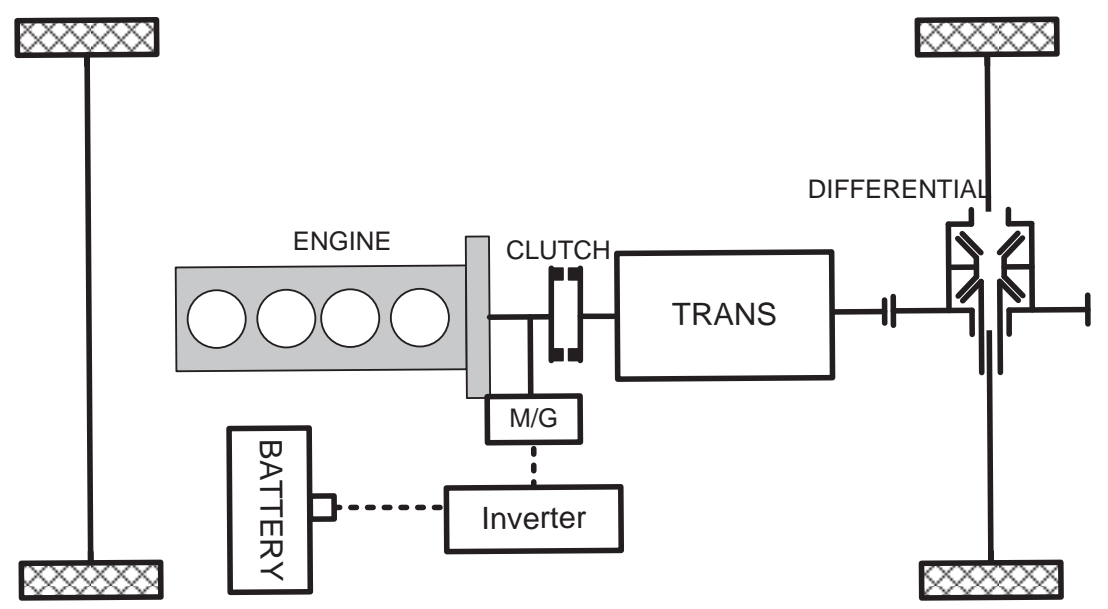

Figure 2-2: Parallel HEV architecture.

\subsubsection{Belt Alternator Starter}

A Belt Alternator Starter (BAS) system integrates an electric motor, power electronics, electric control unit and a battery. The BAS can increase vehicle fuel economy, reduce tail pipe emissions and improve the vehicle performance [15]. A BAS system achieved up to $10 \%$ improvements in fuel economy for city drive cycles [16]. In a BAS hybrid electric vehicle, the belt driven alternator is replaced with an electric motor, which also serves as a generator. A BAS enables the vehicle to perform engine stop-start, electromechanical launch assist, regenerative braking, high power generation, and other functionality without requiring large changes to the vehicle. When the electric motor is driven by the engine, it is acting as a generator to charge a separate high voltage battery. When the engine needs to be started, the motor can drive the engine via the accessory belt, and cranks the engine instead of using the starter motor.

In a BAS hybrid vehicle, the electric motor is commonly made larger than the regular starter motor so more torque can be generated to start the engine quicker and make it possible to realize the engine stop-start operation. The stop-start function of the 
BAS hybrid vehicle stops fueling the engine while the vehicle is at rest at traffic lights. The BAS hybrid takes advantage of the least modification of the conventional powertrain and doesn't have a complicated hybrid control system. However, the BAS hybrid vehicle improves the fuel economy in a small range of two to five percent, which mostly affects the city mileage. The BAS hybrid vehicle has hardly any effect on the highway mileage. Another disadvantage of the BAS hybrid is that the driver notices the engine starting and stopping.

A BAS+Rear Traction Assistance (RTA) hybrid vehicle, shown in Figure 2-3, adds a rear traction motor to assist the propulsion and capture the braking energy. The rear traction motor can assist the vehicle for hard acceleration, hill climbing and towing. It can also achieve e-drive in a limited vehicle speed range. The rear traction motor can work as a generator to capture the braking energy. However, a more complicated hybrid control algorithm is needed to realize the additional control functions.

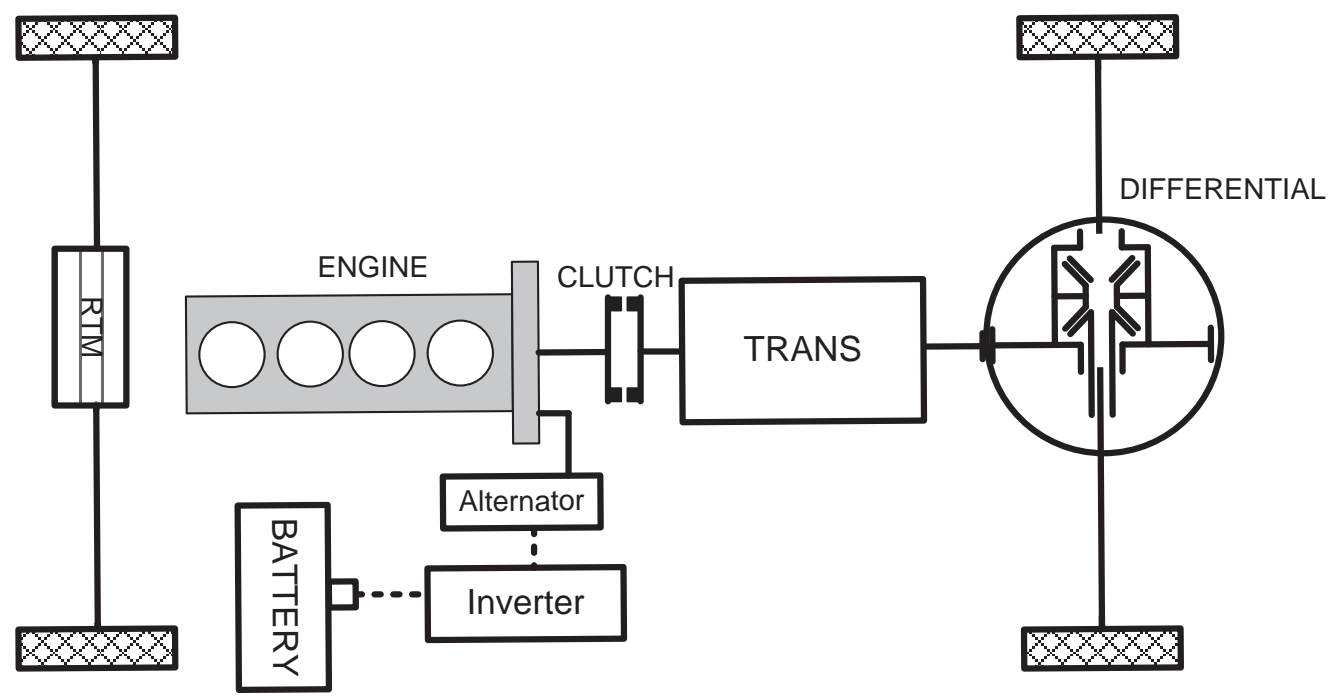

Figure 2-3: BAS+RTA hybrid schematic. 


\subsection{4 $\quad \underline{\text { Power Split HEVs }}$}

An HEV can be categorized by the mechanical connections of electric motors as series, parallel and power split. In a series HEV, only the EM is connected to the final drive and drives the wheels directly, while the IC engine operates to charge the ESS. In a parallel HEV, both the EM and IC engine are connected to the powertrain, and they can drive the wheels at the same time or separately. In a power split HEV, the power split device combines the IC engine and two EMs with a planetary gear set and allows the vehicle to operate with both features of a series HEV and a parallel HEV.

\subsubsection{1 $\quad$ History}

The power split device has a long history since it was invented and tested in the United States in the 1930s. The first patented invention of a power split device was designed by Baruch Berman, et al. in 1971 [17]. The early application of the power- split device was widely used on lawn tractors as a hydrostatic power split transmission.

The milestone of the power split application in passenger vehicles was the production of the Toyota Prius in 1997. The Prius was first introduced in Japan in 1997, and was subsequently on sale worldwide in 2001. The Prius in production during 19972001 was the first generation of the Toyota Hybrid System (THS). The Prius became the world's first mass-produced HEV and was classified as an ultra-low emission vehicle by the California Air Resources Board (CARB). Later Toyota released the second generation Prius with a further developed THS system, named the Toyota Synergy Drive system. A similar hybrid system was also applied in Toyota sport utility vehicle (SUV), the Highlander and the Lexus RX 400h in 2006 and in the Camry and Lexus GS 450h sedans in 2007. The 2011 Highlander Hybrid improved fuel economy to 28 MPG in both city 
and highway. Ford was licensed to use some of the Toyota's hybrid technologies by trading some of its diesel and direct-injection engine technologies. Ford used the licensed technology similar to the Prius to produce the first hybrid SUV to hit the market in 2004. According to the EPA, the first generation Ford Escape Hybrid was 70\% more efficient than the regular Escape. The second generation Ford Escape Hybrid has been produced since 2009. The EPA rated the fuel economy for the 2010 FWD Escape Hybrid at 34 MPG for city and 31 MPG for highway, which has decent fuel economy compared with the non-hybrid Escape rated at 23 MPG for city and 28 MPG for highway.

A 2-Mode EVT, Allison EP 40/50, with one input-split EVT mode and one compound-split EVT mode was first introduced for transit buses in 2003. Since being introduced, Allison hybrid transmissions were installed in over 600 buses and have traveled over 100 million miles around the world. In 2005, General Motors (GM), Daimler, Chrysler LLC and BMW joined together to develop 2-Mode hybrid technology, known as advanced hybrid system 2. In a 2-Mode transmission, three planetary gear sets are used for rear wheel drive and two planetary gear sets are used for front wheel drive. The 2-Mode hybrid system was introduced in the 2008 model year full-sized Chevrolet Tahoe and GMC Yukon SUVs, followed by the Cadillac Escalade and GMC Sierra in 2009. BMW applied the 2-Mode hybrid system in the 2009/2010 X6 ActiveHybrid, as well as the 2009/2010 Mercedes Benz ML 450 hybrid vehicles.

\subsubsection{Input Split (1-mode)}

In motor vehicles, the transmission is used to reduce engine speed to the slow wheel speed, increasing the output torque. Commonly, the transmission has multiple gear ratios, which allows multiple speed ratios to reduce the engine speed and increase the 
output torque. The transmission ratio shift can be done manually or automatically, known as manual transmissions or automatic transmissions. The need for multiple transmission ratios is a consequence of the characteristics of the IC engine and the unpredicted road conditions. The engine typically operates at high speed and low torque, while wheels rotate at low speed and high torque.

The Continuously Variable Transmission (CVT) is a transmission with the ability to change the speed ratio continuously within a given range, providing an infinite number of possible ratios. This can provide better engine fuel economy if the engine can be operated at a constant speed with less transient behavior. Therefore, the engine operating speed is separated from the vehicle speed by adjusting the transmission ratio.

In a power split HEV, an EVT adjusts electric motor speeds to control the transmission speed ratio to achieve the CVT feature. An EVT is commonly designed around a planetary gear set, which works like a differential to split engine power. Most of the engine power is carried through the planetary gear set (mechanical path), while the remaining portion of engine power is converted to electrical energy by electric motors/generators (M/Gs).

The core of most power split HEVs is a planetary gear set, shown in Figure 2-4. A planetary gear set has four rotating parts: sun, ring, pinion, and carrier. The center element is the sun gear, and the outer element is the ring gear. The intermediate parts are 3 or 4 pinions held as a unit by a carrier. The pinions are intermediate elements that do not change the speed ratio between the sun gear and the ring gear. So the ring, sun and carrier are the main rotating parts. The ring, sun or carrier can be configured with 
different combinations as the input shaft, output shaft, or held stationary. Different configurations determine different gear ratios between them.

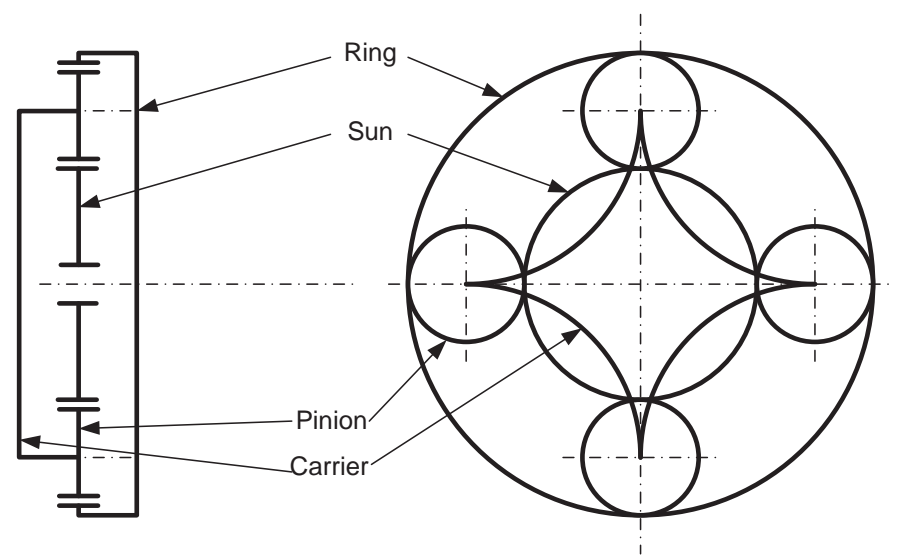

Figure 2-4: Planetary gear set device.

The connection configuration of HEV sources to the planetary gear set determines the power specifications of the vehicle. In the Toyota Hybrid System II (THS-II), shown in Figure 2-5, the sun gear is connected to the Motor/Generator 1(M/G1), the carrier is connected to the IC engine, and the ring gear is connected to the final drive, coupled with the Motor/Generator 2 (M/G2). The vehicle can be powered by the IC engine, the motor alone or in combination with the engine. The power split HEV with a single planetary gear is known as a 1-mode hybrid because only one EVT mode is achieved. The 1-mode hybrid is also known as an input split, because the input, the engine, is connected to the planetary carrier, and the power flow through the transmission is split by the carrier through a mechanical path and an electrical path. 


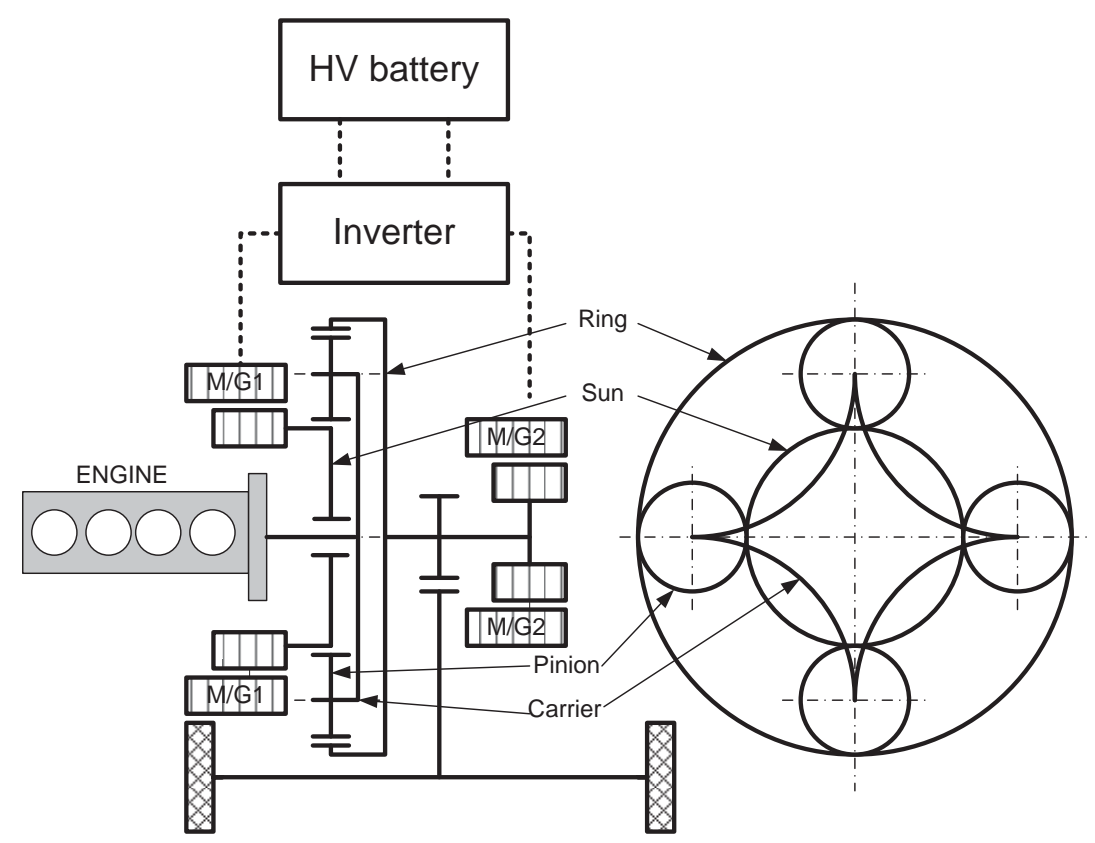

Figure 2-5: 1-Mode HEV layout.

The kinematics of the 1-mode transmission is straightforward. The planetary gear set constructs an EVT, using electric motors to control its speed ratio. In a planetary gear set, the carrier speed is the weighted average speed of the sun gear and the ring gear. In Figure 2-5, the transmission output speed is the weighted average speed of the engine and $M / G 1$, as they are connected to one planetary gear set. The transmission gear ratio is zero when the vehicle is driven by M/G2 with the engine shutdown. The transmission gear ratio is infinite when the engine is running and vehicle stands still. The transmission gear ratio can be anywhere between zero and infinity.

Typically, most of the engine power is carried through the planetary gear set. The rest of the engine power is converted through M/G1 to electrical energy, most often consumed by M/G2 instantly. Output power is added by M/G2, which transfers the electric energy generated from M/G1 back into mechanical power. Therefore, there are two power paths through the transmission from the input to the output. One is the 
mechanical path that directly carries engine power through the transmission. The other one is the electrical path that transfers part of engine power to electric energy by one electric motor, $\mathrm{M} / \mathrm{G} 1$, and then converts back to mechanical power by the other electric motor, M/G2. Power carried through the electric path is less efficient than that through the mechanical path because of power losses during generation and motoring through the battery and the power inverter. At one point where the M/G1 speed is zero, all the engine power is carried through the mechanical path. This is called a "mechanical point", which has the highest power transmission efficiency.

\subsubsection{2-Mode HEV}

The GM 2-Mode FWD transmission is an EVT consisting of two planetary gear sets, four wet-plate clutches for gear selection, one wet-plate damper bypass clutch for vehicle starting and two electric motors/generators, shown in Figure 2-6. The 2-Mode HEV integrates two electromechanical power split modes with four fixed gear ratios, allowing electric assist, regenerative braking, low-speed electric operation and engine starting [18].

Transmission gear selection is achieved by engaging four wet-plate clutches, C13, CB12R, C234 and CB4, see Figure 2-6 [19]. The number of the clutch label shows the clutch engagement status when the corresponding fixed gear is selected. For example, C13 and CB12R are engaged in fixed gear 1; C13 is engaged in fixed gear 1 and fixed gear 3. "CB" indicates the clutch is used as a brake to hold a gear still. The wet-plate Damping Bypass Clutch (DBC) is used for vehicle starting. By locking clutch DBC, the engine is connected to the transmission without a damping effect. 


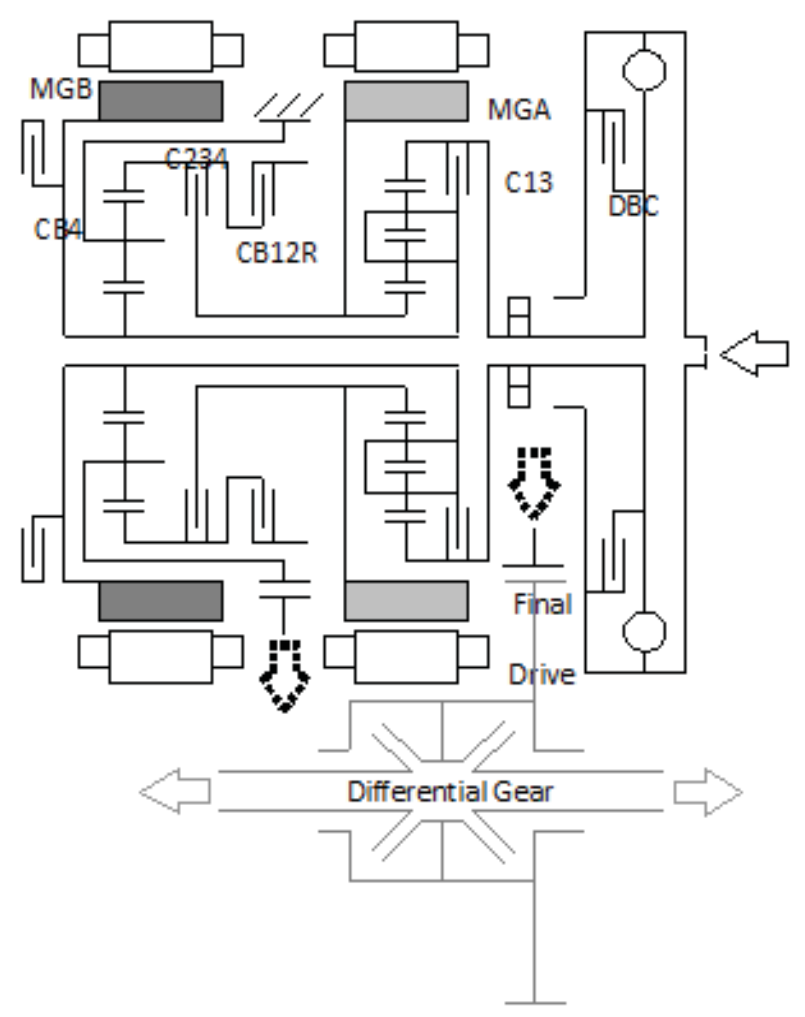

Figure 2-6: 2-Mode transmission diagram [19].

The combination of two power split modes reduces the amount of mechanical power that must be converted to electric power for EVT operation in the high speed range. Figure 2-7 demonstrates mechanical power transmission through a 1-mode EVT with constant engine speed, varying vehicle speed and no battery power contribution. The power through the electrical path increases rapidly as the vehicle speed crosses the mechanical point into the high speed range. Therefore, the vehicle operating efficiency is decreased at higher speed with a 1-mode power split device. In order to decrease the electric power carried through the planetary gear set in the high speed range, another planetary gear set is introduced to provide an extra power split mode, known as a compound split. With the compound split, the electric power is reduced in the high speed range to improve transmission operating efficiency, shown in Figure 2-8 [20]. During 
high speed driving, M/G1 and M/G2 speeds are limited, as well as electrical power generated.

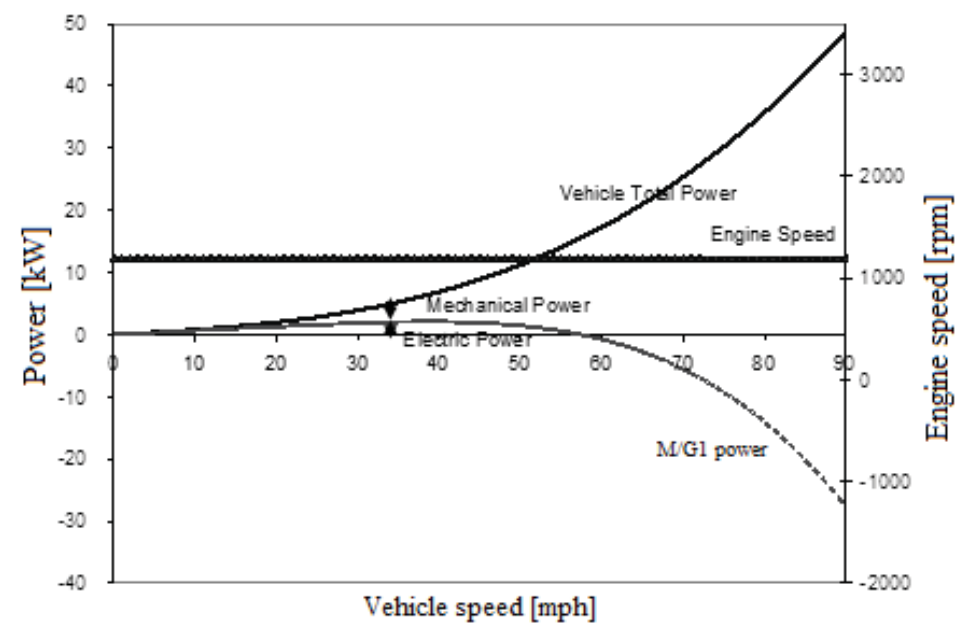

Figure 2-7: Power flow through mechanical and electrical paths [20].

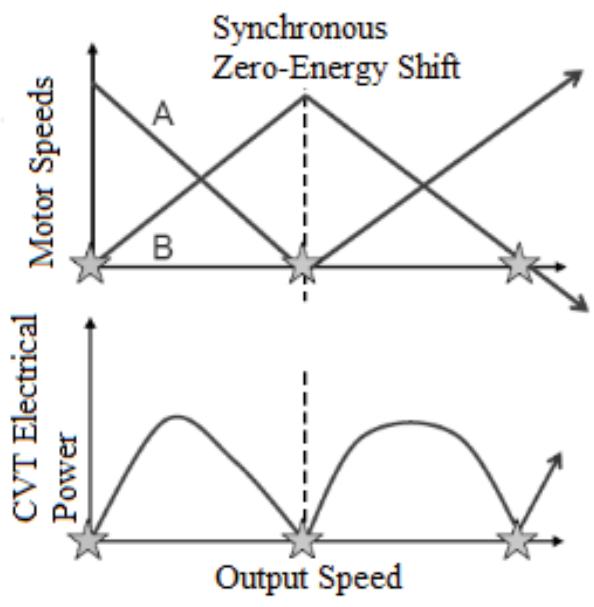

Figure 2-8: 2-Mode EVT driving to limit electrical power [20].

The performance of a 2-Mode hybrid vehicle is improved by increasing the ability of the engine to operate at its peak power through transmission shifting optimization, and using battery power for boosting. With two power split modes, the engine can be 
operated with peak power/torque output independent of the vehicle speed. The input split mode can work in the low speed range, while the compound split mode can work in the high speed range. If extra power is commanded by the driver, the vehicle can be boosted with the battery power to drive the electric motors.

The performance of a 2-Mode hybrid vehicle is further improved with fixed gears introduced in the transmission. The fixed gear modes improve vehicle performance and fuel economy under special demands, such as full throttle, hill climbing and towing, and high speed cruising. Test results showed that fixed gear 1 increases the vehicle traction capability significantly in the range of 10-45 mph [18]. Fixed gear 2 with a ratio of 1.88 : 1is useful for trailering on a grade at highway speeds. Moreover, if battery power is limited or the electrical system is overheated, using EVT modes will limit the vehicle's performance considerably. At this time, using fixed gears can be help to improve the vehicle's performance. Moreover, the use of fixed gears reduces the total amount of engine energy transferred through the electrical path. The fixed gear 3 with a ratio of 1:1 provides optimum fuel economy for trailering cruise by reducing the need to process power electrically. The fixed gear 4 for regenerative braking with the engine on at high vehicle speeds eliminates energy recycle through M/G2 [18]. However, the use of fixed gears was not explored in this research because of the complexity and accuracy of the engine torque/speed control required in fixed gears.

The 2-Mode transmission was initially implemented in transit buses [21] with improved fuel mileage, reduced emissions, and overall sound levels similar to that of passenger cars. The National Renewable Energy Laboratory (NREL) studies showed that 
the 2-Mode buses had up to 30\% higher fuel economy and reduce total operating costs by as much as $15 \%$ [22].

The GM 2-Mode HEV has a number of advantages when compared to series hybrid and parallel hybrid. Since this architecture can run solely from the electric motors or from the engine or from the combination of both, the efficiency level for both highway and city driving is generally higher. The engine can shut down during city driving to keep emissions down and fuel economy at a premium. The Rear Wheel Drive (RWD) Hybrid Tahoe improved its fuel mileage by 50\% in city driving according to EPA figures (21 MPG). It also had a 30\% overall city/highway fuel efficiency improvement. The 2009 FWD 2-Mode hybrid Saturn Vue prototype delivered up to a 50\% percent improvement in EPA unadjusted composite fuel economy [23].

The GM 2-Mode Hybrid has a number of advantages compared to a 1-mode hybrid. The 2-Mode hybrid improves fuel economy more in highway driving compared with a 1-mode hybrid, like the Ford Escape Hybrid and Toyota Highlander Hybrid. In the 2-Mode hybrid, the compound split mode (Mode 2) provides better vehicle fuel economy in highway operation by limiting the engine power travelling through the electrical path. According to the EPA fuel economy rating in city cycle/HWFET with modification, the Ford Escape Hybrid and Toyota Highlander Hybrid are 34/31 MPG and 27/25 MPG respectively. It is easy to find out that fuel economy in highway driving is even lower than in city. The reason is that there is not much regenerative braking energy available in highway driving and more engine power is carried through the electrical path to the wheels in a 1-mode hybrid vehicle. With the model built in PSAT, the 2-Mode front wheel drive Saturn Vue hybrid delivered 35 mph highway driving, which intensely 
improves fuel economy on the highway compared with the Escape Hybrid and Highlander Hybrids.

\subsection{Research Mule Vehicle}

The 2009 Saturn Vue was the mule vehicle for the proposed 2-Mode HEV. The stock 2009 Saturn Vue was a four door, five passenger vehicle equipped with four wheel disk brakes. It had a range of 360 miles on one 19 gallon tank of gasoline and a cargo capacity of $29 \mathrm{ft}^{3}$. The stock Saturn Vue was equipped with 2.4 L Ecotec engine with variable valve timing. It produced $164 \mathrm{hp} / 122 \mathrm{~kW}$ at 6,300 rpm and $160 \mathrm{ft} \cdot \mathrm{lb} / 217 \mathrm{Nm}$ of torque at 4,500 rpm. The Vue had a sticker fuel economy of 19/26 MPG in Urban Dynamometer Driving Cycle (UDDS)/Highway Fuel Economy Driving Schedule (HWFET). It fell under the EPA's Tier II Bin 5 emissions category and had Well-toWheel (WTW) GHG emissions of $246 \mathrm{~g} / \mathrm{km}$. A baseline vehicle longitudinal model was studied to evaluate the vehicle power and the torque requests for UDDS/HWFET test cycles, the 0-60 mph and 0-70 mph acceleration, as well as the available regenerative braking energy.

\subsection{Summary}

A comprehensive review of the hybrid vehicle powertrains has been presented. The review of the hybrid electric vehicle designs was used to direct the selection of the hybrid vehicle powertrain.

Analytic research was performed on the power split device including the dynamic specifications of the 1-mode and 2-Mode transmissions. The operating theory of 1-mode and 2-Mode transmissions was present separately. The advantages and the disadvantages of the two power split transmissions were listed to compare the operation efficiency and 
control complexity. The 2-Mode transmission operation modes were discussed using lever diagrams and mathematical equations.

The 2-Mode hybrid electric vehicle is capable of improving the overall energy efficiency in the high speed range compared to the 1-Mode hybrid electric vehicle. The power split mode in the 2-Mode hybrid electric vehicle limits the electric power flow in the high speed range and improves the overall energy efficiency. However, the 2-Mode hybrid electric vehicle requires the control of more complex hybrid powertrain components and brings a challenge to the control system design and implementation. 


\section{CHAPTER 3}

\section{VEHICLE ARCHITECTURE SELECTION PROCESS}

Based on the literature review, the hybrid electric vehicle selection included selecting candidate HEV architectures, making scoring criteria, analyzing simulation results and finalizing the hybrid powertrain components. The HEV architecture selection was not the focus of the research, but this chapter summarizes the selection process to provide background of work leading to this dissertation.

\subsection{Vehicle Architecture Consideration}

Based on the literature review, the availability of major drivetrain components and an initial assessment of the ability to implement various architectures, three candidate architectures were selected and evaluated:

- 2-Mode Hybrid Electric Vehicle

- Belt Alternator Starter plus Rear Traction Assist (BAS+RTA)

- Parallel Plug-in Hybrid Electric Vehicle

\subsection{1 $\quad$ Vehicle Technical Specifications}

The success of any vehicle relies on a balanced tradeoff between performance, fuel efficiency, utility, ride and drive quality and convenience features. To guide design, a set of vehicle technical specifications (VTS) and requirements were set as hybrid vehicle design targets, listed in Table 3-1. The VTS had a minimum performance criterion and a target criterion. The VTS evaluated the hybrid vehicle from the aspects of fuel efficiency, environmental impact and performance. The combined fuel economy on the UDDS and HWFET driving cycles were used to calculate the Corporate Average Fuel 
Economy (CAFE) unadjusted fuel economy in the simulation. A comprehensive method of evaluating the environmental impact was modeled with the Greenhouse Gases, Regulated Emissions, and Energy Use in Transportation Model (GREET) software [24] from WTW. Vehicle performance was evaluated based on the acceleration capability and the towing capacity. Some convenience features were set such as range, cargo capacity and passenger capacity.

Miles per gallon gasoline equivalent (MPGge) is a measure of the average distance traveled per unit of energy consumed using gasoline. MPGge is used by the U.S. EPA to compare energy consumption of alternative fuel (diesel, bio-fuels, natural gas, etc.) vehicles, plug-in electric vehicles and other advanced technology vehicles with the fuel economy of conventional internal combustion vehicles expressed as miles per U.S. gallon gasoline.

In the Table 3-1, the target performance criteria defined the target vehicle specifications in fuel economy, performance and tailpipe emissions. The target vehicle projected the fuel economy of 35 MPGge and the PEU less than or equal to $0.4 \mathrm{kWh} / \mathrm{km}$. The target vehicle aimed to achieve EPA Tier II Bin 5 tailpipe emissions standard and GHG emissions less than or equal to $150 \mathrm{~g} / \mathrm{km}$. The target vehicle could drive 200 miles with a full tank of fuel. For vehicle performance, the target vehicle was designed to tow $680 \mathrm{~kg}$ load and to drive at $45 \mathrm{mph}$ on a 3.5\% grade. The target vehicle was also expected to accelerate from 0 to $60 \mathrm{mph}$ in $8 \mathrm{~s}$ and from $50 \mathrm{mph}$ to $70 \mathrm{mph}$ in $6 \mathrm{~s}$. 
Table 3-1: Vehicle technical specifications.

\begin{tabular}{|c|c|c|c|}
\hline $\begin{array}{l}\text { Specification } \\
\text { Items }\end{array}$ & \begin{tabular}{|l|} 
Min. Performance \\
Criteria
\end{tabular} & $\begin{array}{l}\text { Target Performance } \\
\text { Criteria }\end{array}$ & \begin{tabular}{|l} 
Prototype Mule \\
Vehicle
\end{tabular} \\
\hline $\begin{array}{l}\text { Fuel Economy, } \\
\text { CAFE Unadjusted, } \\
\text { Combined }\end{array}$ & $\begin{array}{l}\geq 8.3 \mathrm{l} / 100 \mathrm{~km} \\
\text { (28.3 MPGge) }\end{array}$ & 35 MPGge & 28 MPGge \\
\hline $\begin{array}{l}\text { Petroleum Energy } \\
\text { Usage (PEU) }\end{array}$ & $\leq 0.65 \mathrm{kWh} / \mathrm{km}$ & $\leq 0.40 \mathrm{kWh} / \mathrm{km}$ & $0.73 \mathrm{kWh} / \mathrm{km}$ \\
\hline $\begin{array}{l}\text { WTW GHG } \\
\text { Emissions }\end{array}$ & $\leq 217 \mathrm{~g} / \mathrm{km}$ & $\leq 150 \mathrm{~g} / \mathrm{km}$ & $217 \mathrm{~g} / \mathrm{km}$ \\
\hline $\begin{array}{l}\text { Tailpipe } \\
\text { Emissions }\end{array}$ & EPA Tier II Bin 5 & EPA Tier II Bin 5 & EPA Tier II Bin 5 \\
\hline Range & $\begin{array}{l}\geq 320 \mathrm{~km}(200 \\
\text { miles })\end{array}$ & $\begin{array}{l}\geq 320 \mathrm{~km} \mathrm{(200} \\
\text { miles) }\end{array}$ & \begin{tabular}{|l}
$580 \mathrm{~km}(360$ \\
miles)
\end{tabular} \\
\hline Towing Capacity & 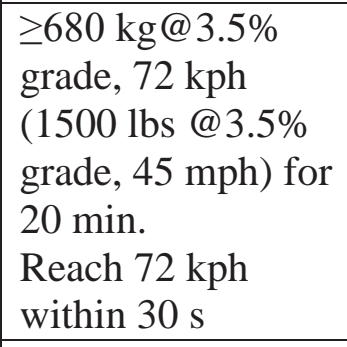 & $\begin{array}{l}\geq 680 \text { kg@3.5\% } \\
\text { grade, } 72 \mathrm{kph}(1500 \\
\text { lbs @3.5\% grade, } \\
45 \mathrm{mph}) \text { for } 20 \text { min. } \\
\text { Reach } 72 \mathrm{kph} \\
\text { within } 30 \mathrm{~s}\end{array}$ & $\begin{array}{l}\geq 680 \mathrm{~kg} @ 3.5 \% \\
\text { grade, } 72 \mathrm{kph} \\
(1500 \mathrm{lbs} @ 3.5 \% \\
\text { grade, } 45 \mathrm{mph} \text { ) } \\
\text { for } 20 \mathrm{~min} . \\
\text { Reach } 72 \mathrm{kph} \\
\text { within } 30 \mathrm{~s} \\
\end{array}$ \\
\hline $\begin{array}{l}\text { Acceleration (0- } \\
60 \mathrm{mph})\end{array}$ & $\leq 14 \mathrm{~s}$ & $8 \mathrm{~s}$ & $10.6 \mathrm{~s}$ \\
\hline $\begin{array}{l}\text { Acceleration (50- } \\
70 \mathrm{mph} \text { ) }\end{array}$ & $\leq 10 \mathrm{~s}$ & $6 \mathrm{~s}$ & $5 \mathrm{~s}$ \\
\hline Vehicle Mass & $\leq 5,000 \mathrm{lbs}$ & $<4,300 \mathrm{lbs}$ & 3,875 lbs \\
\hline Cargo Capacity & $0.83 \mathrm{~m}^{3}$ & $0.83 \mathrm{~m}^{3}$ & $0.83 \mathrm{~m}^{3}$ \\
\hline $\begin{array}{l}\text { Passenger } \\
\text { Capacity }\end{array}$ & $\geq 4$ & 5 & 5 \\
\hline
\end{tabular}

The baseline vehicle to which the research vehicle was compared was a crossover utility vehicle. The crossover utility vehicle was a popular alternative for consumers who needed large cargo volume and also wanted better fuel economy compared to a full-size SUV. The Saturn Vue was a four door, five-passenger crossover utility vehicle. The unmodified stock vehicle had a range of 360 miles from its 19 gallon gasoline tank and a cargo capacity of $29.3 \mathrm{ft}^{3}$. The stock crossover utility vehicle was equipped with a $2.4 \mathrm{~L}$ Ecotec inline 4 cylinder spark-ignited (SI) engine with Variable Valve Timing (VVT). It 
produced 164 horsepower at 6,300 rpm and $160 \mathrm{lb} \cdot \mathrm{ft}$ of torque at 4,500 rpm. The stock Vue achieved fuel economy of 19 MPG in UDDS/26 MPG in HWFET. It fell under the EPA's Tier II Bin 5 emissions category with GHG emissions of $246 \mathrm{~g} / \mathrm{km}$.

In order to choose the best hybrid vehicle architecture, engineering analysis, modeling and simulations were performed using PSAT, GREET and other tools for each hybrid vehicle candidate architecture. PSAT applied a forward-looking modeling method to simulate the real-world vehicle powertrain and control system to meet the requirements of automotive engineering [25], [26]. It has been proven to be a reliable simulation tool to predict vehicle fuel economy and performance [27].

\subsubsection{1 $\quad$ Fuel Economy}

The vehicle fuel economy has become more and more important for customers when buying new vehicles. The annual New Vehicle Customer Study has been conducted since the 1970s by Maritz Research, one of the world's largest marketing research firms. The latest study, reaching approximately 200,000 consumers in 2011, showed that 42 percent of people surveyed said fuel economy is "extremely important" in their decision to purchase new 2011 models. This represented a 13.5 percent increase versus 10 years ago [28]. Furthermore, 37 percent indicated they expected fuel economy would have the "greatest impact" on their next new vehicle purchase. Millennial placed an even greater importance on fuel economy, with 46 percent saying fuel economy was "extremely important" in the new vehicle purchase decision - the greatest percentage among all age groups [28]. Also, 41 percent said fuel economy would be the top factor in their next vehicle purchase [28]. 
Automotive manufacturers test their own vehicle's fuel economy and report the results to the EPA. Fuel economy provided by automakers is tested on both the UDDS and HWFET drive cycles by a trained driver under controlled laboratory conditions. The EPA verifies the manufacturer's fuel economy using a standardized test procedure specified by federal law [29, 30]. EPA reviews the results and confirms about 10-15 percent of them through their own tests at the National Vehicles and Fuel Emissions Laboratory.

Gasoline equivalent fuel economy was determined for the UDDS and the HWFET [31], [32] and used to compute the CAFE unadjusted combined fuel economy, which was calculated with Eq. 3-1.

$$
m p g_{c o m b}=0.55 \cdot m p g_{U D D S}+0.45 \cdot m p g_{H W F E T}
$$

Where: $m p g_{c o m b}$ is the unadjusted combined fuel economy; $m p g_{U D D S}$ is the UDDS fuel economy; $m p g_{H W F E T}$ is the HWFET fuel economy.

The UDDS drive cycle, shown in Figure 3-1, is also called the U.S. Federal Test Procedure -72 or LA-4 cycle. The cycle simulates an urban route of $12.1 \mathrm{~km}$ (7.5 miles) with frequent stops. The maximum speed is $91.2 \mathrm{kph}(56.7 \mathrm{mph})$ and the average speed is $31.5 \mathrm{kph}$ (19.6 mph). The cycle consists of three phases: (1) cold start phase of $505 \mathrm{~s}$ (5.8 $\mathrm{km}$ at $41.2 \mathrm{~km}$ average speed); (2) transient phase of $864 \mathrm{~s}$ and (3) hot start phase of 505 s. 


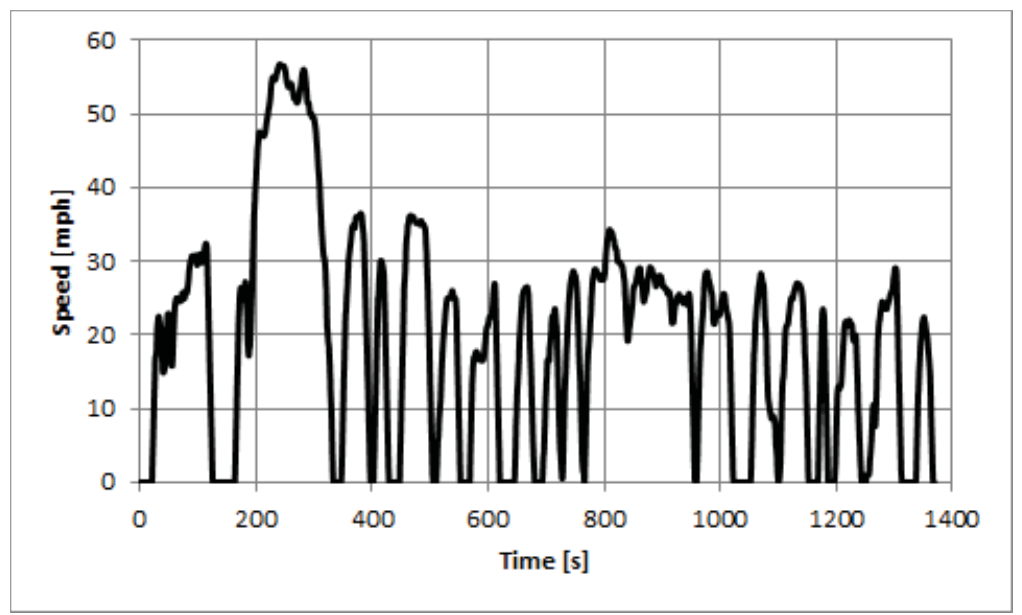

Figure 3-1: UDDS drive cycle [31].

The HWFET drive cycle, shown in Figure 3-2, represents highway driving conditions less than $60 \mathrm{mph}$. The cycle duration is $765 \mathrm{~s}$ with a total distance of 10.3 miles (16.5 km) and average speed of $48.3 \mathrm{mph}(77.7 \mathrm{kph})$.

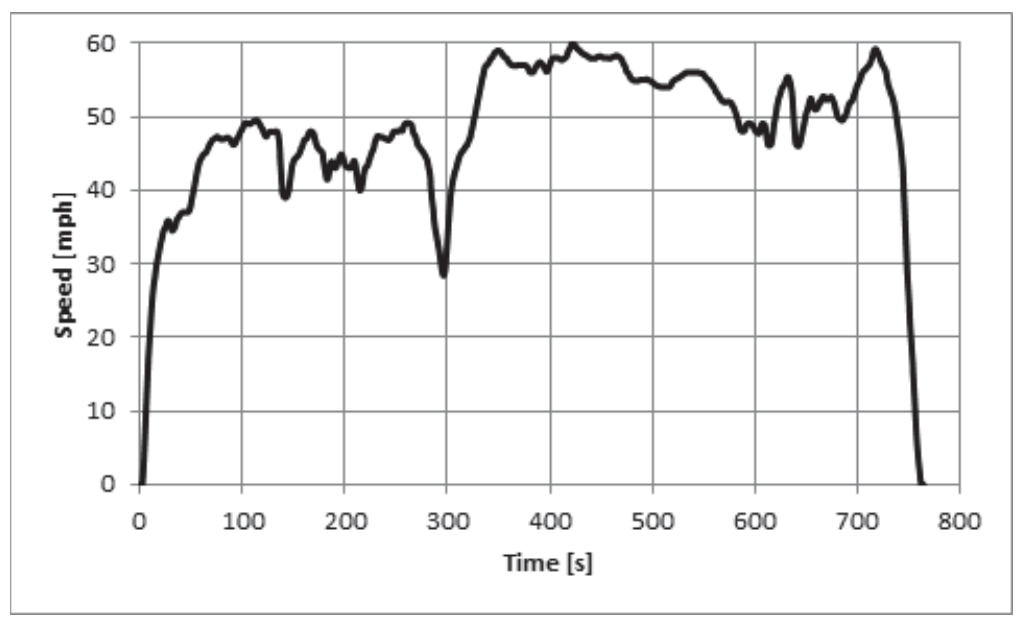

Figure 3-2: HWFET drive cycle [32].

The fuel economy calculation for plug-in hybrid electric vehicles (PHEVs) differs from the electrical grid independent hybrid electric vehicles. For PHEVs, the electricity charged to the high voltage battery from the electrical grid needs to be considered. It requires the inclusion of Charge Depleting (CD) and Charge Sustaining (CS) operation, 
and the use of a utility-factor (UF) to properly combine the vehicle's operating modes using representative driving statistics [33], [34]. The Society of Automotive Engineers (SAE) J1711 standard was used to determine the drive cycle fuel economy with Eq. 3-2.

$$
m p g_{c y c l e}=\left(\frac{0.5}{m p g_{C D, U F}}+\frac{0.5}{m p g_{C S}}\right)^{-1}
$$

Where: $m p g_{c y c l e}$ is test cycle fuel economy; $m p g_{C S}$ is charge sustaining fuel economy; $m p g_{C D, U F}$ is charge depleting fuel economy considering the utility factor.

The CD and CS modes are equally weighted. The $m p g_{C D, U F}, m p g_{C D}$ and the $m p g_{C S}$ were calculated with Eq. 3-3, Eq. 3-4 and Eq. 3-5.

$$
\begin{gathered}
m p g_{C D, U F}=\frac{1}{\frac{U F}{m p g_{C D}}+\frac{1-U F}{m p g_{C S}}} \\
m p g_{C D}=\frac{\text { Dist }_{C D}}{V_{\text {fuel,CD }}+\frac{E_{\text {charge }}}{E_{\text {fuel }}}} \\
m p g_{C S}=\frac{\text { Dist }_{C S}}{V_{\text {fuel,CS }}}
\end{gathered}
$$

Where: $m p g_{C D}$ is charge depleting fuel economy; $m p g_{C S}$ is charge sustaining fuel economy; $\operatorname{Dist}_{C D}$ is charge depleting driving distance; $D i s t_{C S}$ is charge sustaining driving distance; $V_{f u e l, C D}$ is the volume of fuel consumed in gallons; $E_{\text {charge }}$ is the consumed electrical recharge energy in $\mathrm{kWh} ; E_{\text {fuel }}$ is a constant equaling $33.44 \mathrm{~kW} / \mathrm{gal}$ for gasoline and $37.95 \mathrm{~kW} /$ gal for diesel.

Then the fuel economy figures were evaluated for both the UDDS and HWFET schedules. The combined fuel economy was calculated from Eq. 3-1.

The UF was estimated with Eq. 3-6. 


$$
\begin{aligned}
U F=1-\exp \{ & -\left[C 1 *\left(\frac{x}{\text { norm_dist }}\right)+C 2 *\left(\frac{x}{\text { norm_dist }}\right)^{2}+\ldots\right. \\
& \left.\left.+C 9 *\left(\frac{x}{\text { norm_dist }}\right)^{9}\right]\right\}
\end{aligned}
$$

Where norm_dist is the normalized distance that the vehicle is driven in one day.

The norm_dist has a range from 0.5 mile to 438 miles.

The parameters of C1 to C9 can be found in Table 3-2.

Table 3-2: UF norm distance parameters.

\begin{tabular}{|l|l|l|l|l|l|l|l|l|}
\hline \hline C1 & C2 & C3 & C4 & C5 & C6 & C7 & C8 & C9 \\
\hline 10.52 & -7.28 & -26.37 & 79.08 & -77.36 & 26.07 & 0 & 0 & 0 \\
\hline
\end{tabular}

The UF can be plotted with the normalized distance, shown in Figure 3-3. The normalized distance was achieved based on a survey conducted by National Highway Traffic Safety Administration (NHTSA) in 2001 [34].

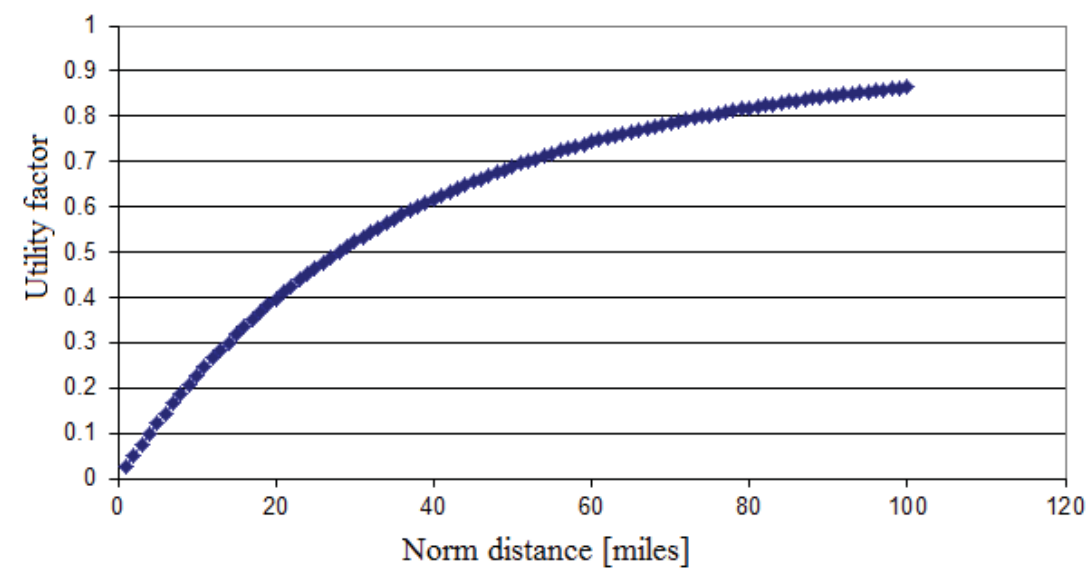

Figure 3-3: The normative utility factor. 


\subsubsection{Well-to-Wheel Effects}

With the results of the simulated vehicle fuel economy, WTW GHG emissions were then computed using GREET 1.8b [24] and PEU was computed based on Well-ToPump (WTP) PEU factors determined by the default allocations for 2015 in GREET 1.8b.

The GREET software fully evaluates energy and emission impacts of advanced vehicle technologies and new transportation fuels from wells to wheels and the vehicle cycle through material recovery and vehicle disposal [24]. GREET allows researchers and analysts to evaluate various vehicle and fuel combinations on a full fuelcycle/vehicle-cycle basis. GREET1.8b was developed as a multidimensional spreadsheet model in Microsoft Excel.

$$
\text { GHG and PEU in units of } g_{\text {CO2_Equivalent } / \mathrm{km}} \text { and } \mathrm{kWh} / \mathrm{km} \text { were determined }
$$

from reference [35], for the UDDS and HWFET, and combined in Eq. 3-7 and Eq. 3-8:

$$
\begin{aligned}
& G H G_{c o m b}=0.55 G H G_{U D D S}+0.45 G H G_{H W F E T} \\
& P E U_{c o m b}=0.55 P E U_{U D D S}+0.45 P E U_{H W F E T}
\end{aligned}
$$

Where: $G H G_{\text {comb }}$ is combined GHG; $G H G_{U D D S}$ is GHG generated in UDDS;

$G H G_{H W F E T}$ is GHG generated in HWFET; $P E U_{\text {comb }}$ is combined PEU; $P E U_{U D D S}$ is PEU consumed in UDDS; PEU HWFET is PEU consumed in HWFET.

\subsubsection{Driving Range}

Hybrid vehicle driving range impacts the size of the fuel tank and the capacity of the high voltage battery. Fuel is the power source of the internal combustion engine. The charging depleting hybrid electric vehicle combines fuel and high voltage battery power to drive the vehicle. A minimum driving range of 200 miles was set for the hybrid 
electric vehicle design. The combined fuel economy from PSAT simulation results were used to calculate the fuel tank volume.

\subsubsection{4 $\quad$ Towing Capacity}

Towing capacity is a test of the vehicle powertrain capability with extra load and road slope. The proposed hybrid powertrain should not only be able to improve fuel economy, but also to maintain towing capability. The towing test required the vehicle to travel at $45 \mathrm{mph}$ on a 3.5\% grade road while towing a load of $680 \mathrm{~kg}$. Extra power needed for towing capacity impacts the terms of mass, velocity and road grade in Eq.

3-9:

$$
P_{\text {Tract }}=\frac{1}{2} \rho_{a i r} C_{d} A v^{3}+\mu_{R R} m g v \cos \alpha+m g v \sin \alpha+m v \frac{d v}{d t}
$$

Where: $P_{\text {Tract }}$ is vehicle traction power; $\rho_{\text {air }}$ is the density of air, which is taken to be $1.3 \mathrm{~kg} / \mathrm{m}^{3} ; C_{d}$ is the vehicle drag coefficient; $A$ is the vehicle frontal area; $v$ is vehicle speed; $\mu_{R R}$ is the coefficient of rolling resistance; $\mathrm{m}$ is the vehicle equivalent mass; $\frac{d v}{d t}$ is vehicle acceleration; $\mathrm{m}$ is vehicle mass; $\mathrm{g}$ is acceleration of gravity, $9.8 \mathrm{~m} / \mathrm{s}^{2}$; $\alpha$ is road grade.

\subsubsection{Acceleration}

Acceleration time is one of the key parameters to evaluate a vehicle's performance. An estimation of the extra average power needed to accelerate a vehicle from 0 to a certain speed v can be calculated with Eq. 3-10:

$$
\frac{\Delta\left(v^{2}\right)}{\Delta t} \approx 2 \frac{P_{\text {Tract }}}{m}
$$

Where: $v$ is speed; $t$ is time; $P_{\text {Tract }}$ is traction power; $m$ is vehicle mass. 


\subsubsection{Braking Distance and Power}

Braking distance was calculated with the braking time, the start speed and the ending speed. An estimation of the braking distance can be calculated with a constant deceleration using Newton's Law as Eq. 3-11:

$$
s=\frac{v_{t}^{2}-v_{0}^{2}}{2 \alpha}
$$

Where: $\mathrm{v}_{0}$ is starting speed; $\mathrm{v}_{\mathrm{t}}$ is ending speed; $\alpha$ is deceleration, which can be calculated with Eq. 3-12.

$$
\alpha=\frac{v_{0}-v_{t}}{\Delta t}
$$

During the braking process of a conventional vehicle, kinetic energy is consumed by the friction, where heat is generated and dissipated in the air. However, part of the braking power can be absorbed by the regenerative braking system in an HEV to charge the battery. An estimation of the braking power can be calculated with Eq. 3-13.

$$
\overline{P_{\text {brake }}}=\frac{\Delta K E}{\Delta t}=\frac{\frac{1}{2} m\left(v_{t}^{2}-v_{0}^{2}\right)}{\Delta t}
$$

Where: $\overline{P_{\text {brake }}}$ is average brake power; $K E$ is kinetic energy.

\subsection{Hybrid Electric Vehicle Architecture Selection Procedure}

Minimum performance standards and evaluation criteria were determined to guide the vehicle powertrain architecture selection process. Table 3-3 lists the evaluation criteria, including the minimum performance, standard target VTS and the weighting factors representing the relative importance of the criteria. 
Table 3-3: Vehicle technical specifications and architecture evaluation criteria.

\begin{tabular}{|c|c|c|c|}
\hline Specification & $\begin{array}{c}\text { Minimum } \\
\text { Performance Criteria }\end{array}$ & Target VTS & Weighting Factor \\
\hline $\begin{array}{l}\text { Fuel Economy, CAFE } \\
\text { Unadjusted, Combined }\end{array}$ & $\begin{array}{l}\geq 8.3 \mathrm{l} / 100 \mathrm{~km} \\
(28.3 \mathrm{MPGge})\end{array}$ & 35 MPGge & 0.21 \\
\hline WTW Petroleum Use & $\leq 0.65 \mathrm{kWh} / \mathrm{km}$ & $\leq 0.40 \mathrm{kWh} / \mathrm{km}$ & 0.21 \\
\hline WTW GHG Emissions & $\leq 217 \mathrm{~g} / \mathrm{km}$ & $\leq 150 \mathrm{~g} / \mathrm{km}$ & 0.21 \\
\hline Tailpipe Emissions & EPA Tier II Bin 5 & EPA Tier II Bin 5 & 0.09 \\
\hline Range & $\geq 320$ km (200 miles) & $\geq 320 \mathrm{~km}$ (200 miles) & 0.01 \\
\hline Towing Capacity & $\begin{array}{c}\geq 680 \text { kg@3.5\%, } 72 \text { kph } \\
\text { (1500 lbs @3.5\%, } 45 \\
\text { mph) for } 20 \text { minutes. } \\
\text { Reach } 72 \text { kph within } 30 \text { s }\end{array}$ & $\begin{array}{c}\geq 680 \text { kg@3.5\%, } 72 \text { kph } \\
\text { (1500 lbs @3.5\%, } 45 \\
\text { mph) for } 20 \text { minutes. } \\
\text { Reach } 72 \text { kph within } 30 \text { s }\end{array}$ & 0.07 \\
\hline Acceleration (0-60 mph) & $\leq 14 \mathrm{~s}$ & $8 \mathrm{~s}$ & 0.09 \\
\hline Acceleration (50-70 mph) & $\leq 10 \mathrm{~s}$ & $6 \mathrm{~s}$ & 0.07 \\
\hline Vehicle Mass & $\leq 2,268 \mathrm{~kg}(5,000 \mathrm{lbs})$ & $<1,950 \mathrm{~kg}(4,300 \mathrm{lbs})$ & 0.03 \\
\hline Cargo Capacity & $0.83 \mathrm{~m}^{3}$ & $0.83 \mathrm{~m}^{3}$ & 0.01 \\
\hline
\end{tabular}

\subsection{1 $\quad \underline{\text { Scoring Criteria }}$}

The candidate architectures were each assigned a score ranging from 0-100 in each category. This score was determined such that the best performing architecture received a score of 100 points. An architecture that performed to the minimum criteria received a score of 20 points. A linear distribution was applied between the minimum performance criteria and the best performing architecture. Any architecture that performed below the minimum criteria received a score of zero in that category. The scoring mechanism is depicted in Figure 3-4. The weighted sum was then determined and the candidate architectures were ranked to determine the best overall powertrain architecture.

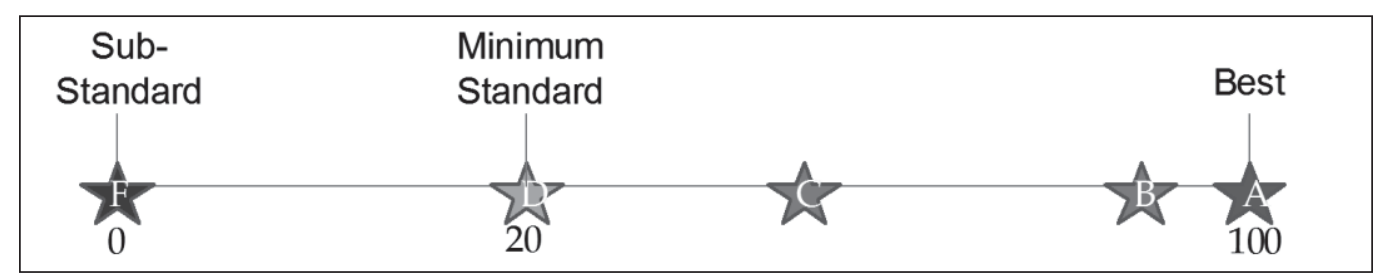

Figure 3-4: Scoring mechanism. 
In order to assign a score to tailpipe emissions a weighted sum equal to $0.25 * \mathrm{HC}+0.5 * \mathrm{NOx}+0.25 * \mathrm{CO}$ was computed. NOx emissions were assigned a higher weight to account for the inherent difficulty of reducing NOx in diesel exhaust.

\subsection{2 $\quad$ Powertrain Configuration Matrix}

The above scoring and ranking system was applied to determine the ultimate architecture selection. Before this final selection method could be applied the 'best' possible design for the candidate architectures had to be determined. The performance of the candidate architectures in each evaluation category was affected by a number of factors such as, fuel type (gasoline, E-85, biodiesel), engine combustion cycle (spark ignition, compression ignition), engine size, type and size of the electric machines, energy storage system type and size and control strategy. Design of Experiments techniques were employed to reduce the size of the simulation matrix.

Table 3-4 shows the major hybrid powertrain components and their relative impact on the performance criteria. The impact value was multiplied by the weighted importance and summed across each row to determine the relative importance of each component in the powertrain design.

Important parameters for each major component were then determined. An example is shown in Figure 3-5. The design space was further refined based on the availability of components and simulations were then performed in PSAT to explore the various configurations for each architecture. As an example, the simulation matrix for the 2-Mode hybrid architecture is shown in Table 3-5. Similar approaches were followed for evaluating the Belt Alternator Starter with Rear Traction Assist and Parallel Architecture Plug-in Hybrid Electric Vehicle Architectures. 
Table 3-4: Component impact matrix.

\begin{tabular}{|c|c|c|c|c|c|c|c|c|c|c|c|}
\hline & 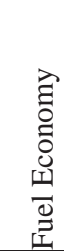 & $\begin{array}{l}\ddot{\Xi} \\
0 \\
0 \\
0 \\
0 \\
0 \\
0\end{array}$ & 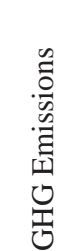 & 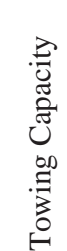 & 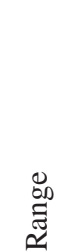 & 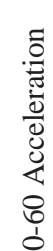 & 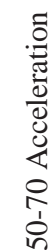 & 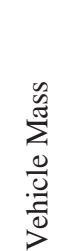 & 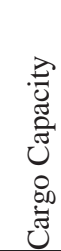 & 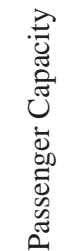 & $\begin{array}{l}\text { Impact } \\
\text { Value }\end{array}$ \\
\hline Fuel Type & 7 & 7 & 7 & 3 & 6 & 3 & 3 & 2 & 0 & 0 & 5.2 \\
\hline Engine & 7 & 7 & 7 & 6 & 6 & 6 & 6 & 5 & 0 & 0 & 6.3 \\
\hline Transmission & 6 & 2 & 2 & 6 & 5 & 5 & 5 & 5 & 0 & 0 & 4.1 \\
\hline Electric Motors & 6 & 4 & 4 & 6 & 5 & 7 & 7 & 5 & 0 & 0 & 5.0 \\
\hline $\begin{array}{l}\text { Energy Storage } \\
\text { System }\end{array}$ & 6 & 4 & 4 & 4 & 6 & 6 & 6 & 5 & 4 & 0 & 4.7 \\
\hline Final Drive & 5 & 3 & 3 & 5 & 5 & 5 & 5 & 0 & 0 & 0 & 3.8 \\
\hline $\begin{array}{l}\text { Auxiliary } \\
\text { Components }\end{array}$ & 3 & 2 & 2 & 0 & 0 & 0 & 0 & 2 & 0 & 0 & 1.4 \\
\hline $\begin{array}{l}\text { Weighted } \\
\text { Importance (\%) }\end{array}$ & 18 & 18 & 18 & 18 & 7 & 7 & 5 & 5 & 3 & 1 & \\
\hline
\end{tabular}

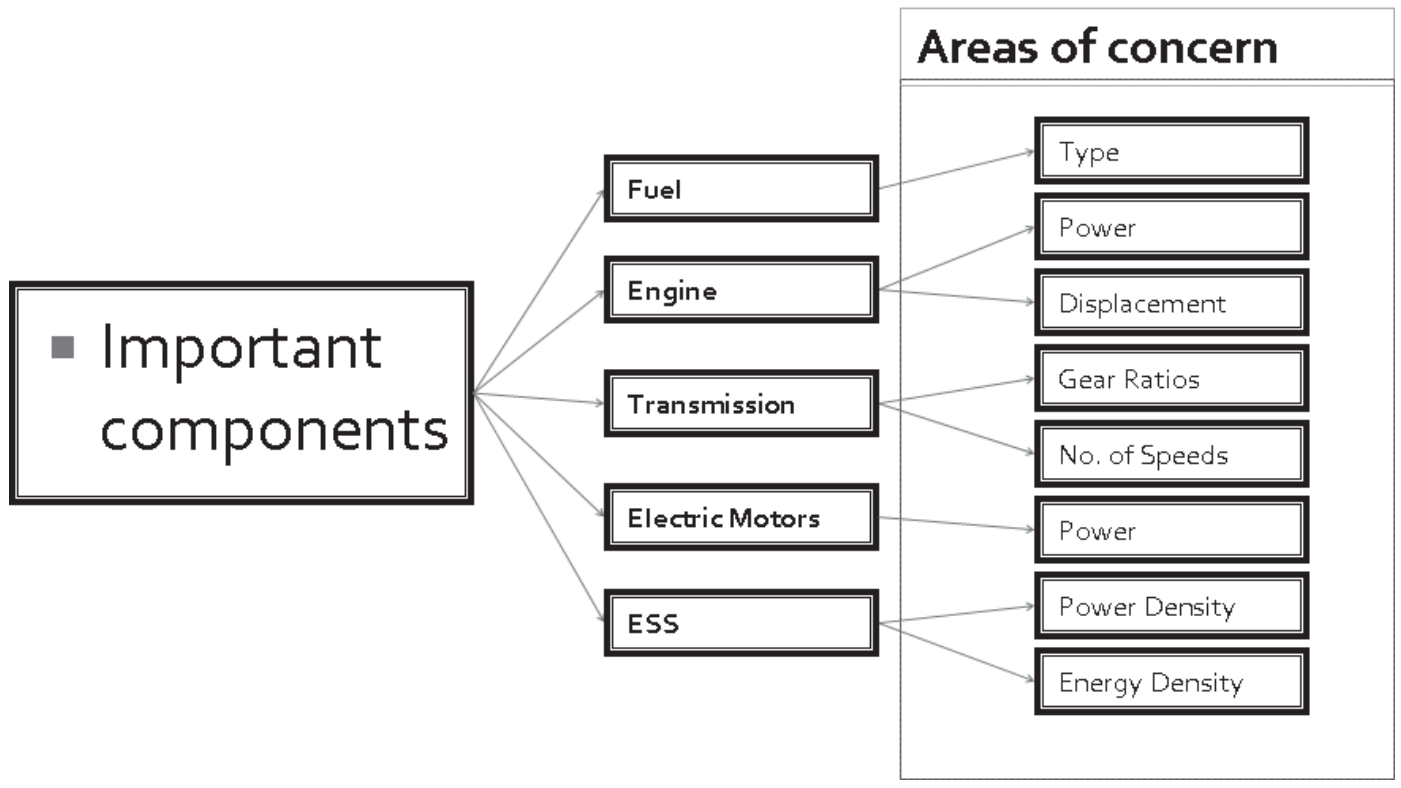

Figure 3-5: Important component parameters. 
Table 3-5: 2-Mode simulation matrix.

\begin{tabular}{|c|c|c|l|l|}
\hline Simulation & Fuel & Engine Size & Motor Size & ESS Size \\
\hline Config.1 & Bio-diesel & $2.0 \mathrm{~L}$ & $\begin{array}{l}\text { M1 }=53 \mathrm{~kW} \\
\text { M2 }=57 \mathrm{~kW}\end{array}$ & $21.6 \mathrm{kWh}, 360 \mathrm{~V}$ \\
\hline Config.2 & Bio-diesel & $1.3 \mathrm{~L}$ & $\begin{array}{l}\text { M1 }=53 \mathrm{~kW} \\
\text { M2 }=57 \mathrm{~kW}\end{array}$ & $12.9 \mathrm{kWh}, 330 \mathrm{~V}$ \\
\hline Config.3 & Bio-diesel & $2.0 \mathrm{~L}$ & $\begin{array}{l}\text { M1 }=53 \mathrm{~kW} \\
\text { M2 }=57 \mathrm{~kW}\end{array}$ & $21.6 \mathrm{kWh}, 360 \mathrm{~V}$ \\
\hline Config.4 & Bio-diesel & $1.3 \mathrm{~L}$ & $\begin{array}{l}\text { M1 }=53 \mathrm{~kW} \\
\mathrm{M} 2=57 \mathrm{~kW}\end{array}$ & $12.9 \mathrm{kWh}, 330 \mathrm{~V}$ \\
\hline Config.5 & Gasoline & $1.8 \mathrm{~L}$ & $\begin{array}{l}\text { M1 }=53 \mathrm{~kW} \\
\mathrm{M} 2=57 \mathrm{~kW}\end{array}$ & $21.6 \mathrm{kWh}, 360 \mathrm{~V}$ \\
\hline Config.6 & Gasoline & $1.6 \mathrm{~L}$ & $\begin{array}{l}\mathrm{M} 1=53 \mathrm{~kW} \\
\mathrm{M} 2=57 \mathrm{~kW}\end{array}$ & $12.9 \mathrm{kWh}, 330 \mathrm{~V}$ \\
\hline Config.7 & Gasoline & $1.8 \mathrm{~L}$ & $\begin{array}{l}\mathrm{M} 1=53 \mathrm{~kW} \\
\mathrm{M} 2=57 \mathrm{~kW}\end{array}$ & $21.6 \mathrm{kWh}, 360 \mathrm{~V}$ \\
\hline Config.8 & Gasoline & $1.6 \mathrm{~L}$ & $\begin{array}{l}\mathrm{M} 1=53 \mathrm{~kW} \\
\mathrm{M} 2=57 \mathrm{~kW}\end{array}$ & $12.9 \mathrm{kWh}, 330 \mathrm{~V}$ \\
\hline Config.9 & E-85 & $1.8 \mathrm{~L}$ & $\begin{array}{l}\mathrm{M} 1=53 \mathrm{~kW} \\
\mathrm{M} 2=57 \mathrm{~kW}\end{array}$ & $21.6 \mathrm{kWh}, 360 \mathrm{~V}$ \\
\hline Config.10 & E-85 & $1.6 \mathrm{~L}$ & $\begin{array}{l}\mathrm{M} 1=53 \mathrm{~kW} \\
\mathrm{M} 2=57 \mathrm{~kW}\end{array}$ & $12.9 \mathrm{kWh}, 330 \mathrm{~V}$ \\
\hline Config.11 & E-85 & $\begin{array}{l}\mathrm{M} 1=53 \mathrm{~kW} \\
\mathrm{M} 2=57 \mathrm{~kW}\end{array}$ & $21.6 \mathrm{kWh}, 360 \mathrm{~V}$ \\
\hline Config.12 & E-85 & $\begin{array}{l}\mathrm{M} 1=53 \mathrm{~kW} \\
\mathrm{M} 2=57 \mathrm{~kW}\end{array}$ & $12.9 \mathrm{kWh}, 330 \mathrm{~V}$ \\
\hline
\end{tabular}

\subsubsection{Fuel Selection and Well-to-Wheel Influence}

Gasoline equivalent fuel economy was determined for the UDDS and HWFET

drive cycles and used to compute the CAFE unadjusted combined fuel economy for each configuration. WTW GHG emissions were then computed using GREET 1.8b [24] and PEU was computed based on Well-to-Pump PEU factors determined by the default allocations for 2015 in GREET 1.8b. Figure 3-6 shows a comparison of the 12 2-Mode configurations in terms of CAFE unadjusted Tank-To-Wheel (TTW) fuel economy, WTW PEU and WTW GHG emissions.

In the 2-Mode HEV configurations, the $1.3 \mathrm{~L}$ diesel engine fueled on a B20 biodiesel blend achieved the highest tank-to-wheels fuel economy followed by 1.6 L gasoline engine. The E-85 fuel yielded substantially lower fuel economy. E-85 showed a 
substantial advantage in WTW petroleum energy use followed by the biodiesel blend. The biodiesel blend had the lowest GHG emissions.

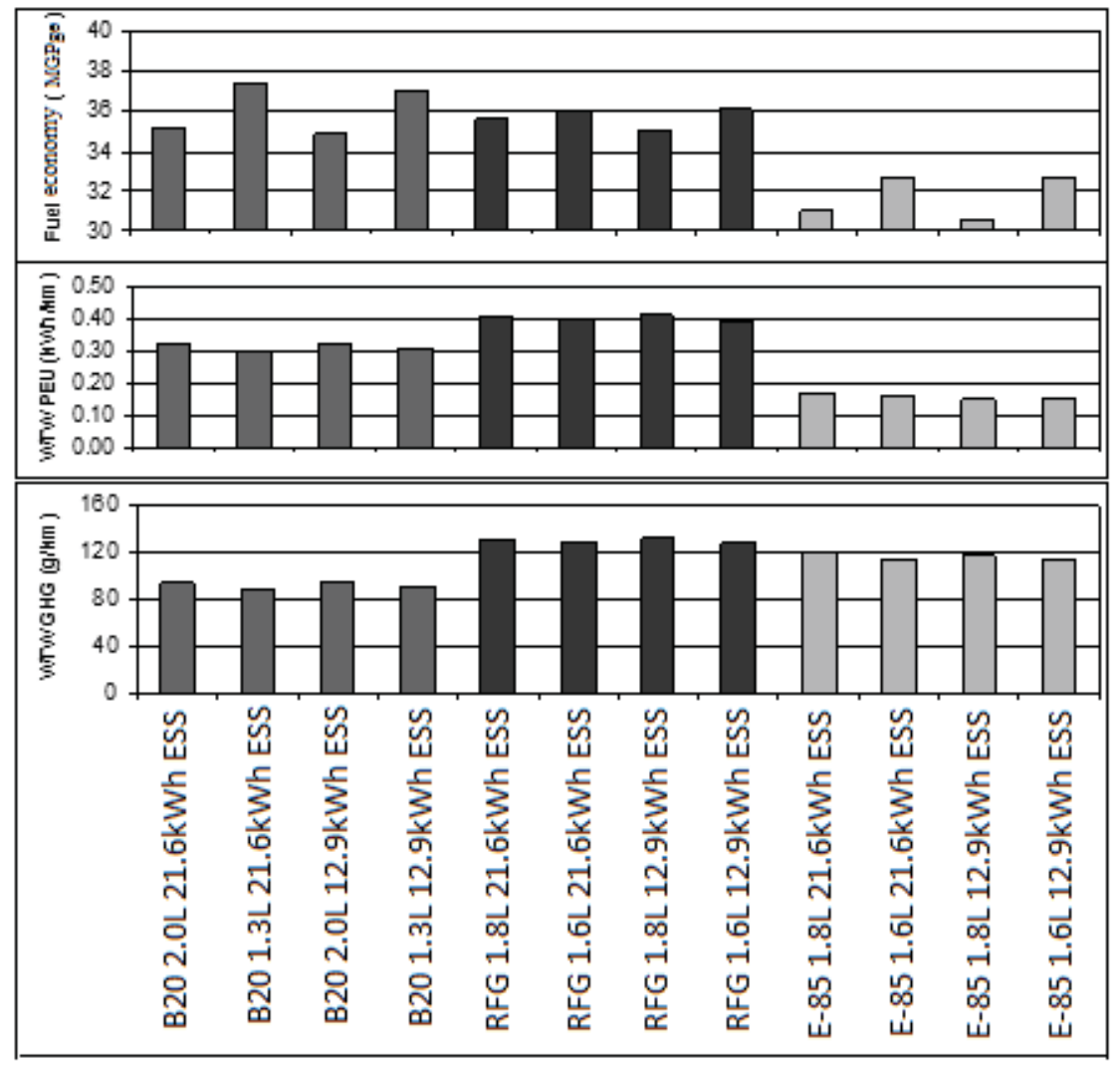

Figure 3-6: 2-Mode fuel economy, PEU and GHG emissions comparison.

\subsection{Summary}

The full results for the selected 2-Mode configurations and the corresponding scores based on the rating methodology described above are presented in Table 3-6. Based on the results the best overall performance was achieved by Configuration \#2 consisting of the $1.3 \mathrm{~L}$ diesel engine fueled with biodiesel and a $12.9 \mathrm{kWh}$ lithium-ion battery system. 
Table 3-6: 2-Mode simulation results.

\begin{tabular}{|c|c|c|c|c|c|c|c|c|c|c|c|c|c|}
\hline & Units & $\# 1$ & $\# 2$ & \#3 & \#4 & $\# 5$ & \#6 & \#7 & $\# 8$ & $\# 9$ & $\# 10$ & $\# 11$ & $\# 12$ \\
\hline $\begin{array}{l}\text { UDDS fuel } \\
\text { economy } \\
\text { (FE) }\end{array}$ & MPGge & 34.9 & 38.1 & 34.6 & 37.7 & 35.5 & 37.6 & 35.5 & 37.6 & 31.0 & 34.0 & 30.9 & 34.1 \\
\hline HWFET FE & MPGge & 35.4 & 38.2 & 35.2 & 37.8 & 35.7 & 34.4 & 34.7 & 34.4 & 31.1 & 31.2 & 30.2 & 31.2 \\
\hline Comb FE & MPGge & 35.1 & 38.1 & 34.9 & 37.8 & 35.6 & 36.1 & 35.1 & 36.1 & 31.0 & 32.7 & 30.6 & 32.7 \\
\hline WTW PEU & $\mathrm{kWh} / \mathrm{km}$ & 0.32 & 0.30 & 0.32 & 0.30 & 0.40 & 0.40 & 0.41 & 0.40 & 0.17 & 0.16 & 0.15 & 0.15 \\
\hline WTW GHG & $\mathrm{g} / \mathrm{km}$ & 94.5 & 89.5 & 96.0 & 90.6 & 130.1 & 129.9 & 131.9 & 128.2 & 120.2 & 114.1 & 117.2 & 113.9 \\
\hline Range & miles & 253 & 269 & 251 & 266 & 256 & 260 & 253 & 260 & 223 & 235 & 220 & 236 \\
\hline Towing & sec & 6.6 & 7.3 & 7.2 & 9.0 & 12.0 & 11.5 & 12.1 & 11.6 & 12.0 & 11.5 & 12.1 & 11.6 \\
\hline 0-60 mph & sec & 7.3 & 8.4 & 7.3 & 10.1 & 9.8 & 9.6 & 10.0 & 10.0 & 9.8 & 9.6 & 10.0 & 10.0 \\
\hline $50-70 \mathrm{mph}$ & sec & 3.9 & 4.3 & 3.9 & 5.1 & 3.9 & 3.9 & 3.9 & 4.1 & 3.9 & 3.9 & 3.9 & 4.1 \\
\hline Weight & lbs & 4,449 & 4,363 & 4,171 & 4,085 & 4,314 & 4,296 & 4,036 & 4,019 & 4,314 & 4,296 & 4,036 & 4,019 \\
\hline Cargo & $\mathrm{m}^{3}$ & 0.79 & 0.79 & 0.83 & 0.83 & 0.79 & 0.79 & 0.83 & 0.83 & 0.79 & 0.79 & 0.83 & 0.83 \\
\hline Passengers & - & 5 & 5 & 5 & 5 & 5 & 5 & 5 & 5 & 5 & 5 & 5 & 5 \\
\hline Score & - & 83.0 & 89.9 & 82.8 & 86.6 & 75.3 & 77.2 & 74.2 & 78.1 & 69.0 & 76.9 & 70.9 & 77.8 \\
\hline
\end{tabular}

Table 3-7 shows the comparison results of the best performance configuration among the 2-Mode, BAS+RTA and Plug-in Hybrid Electric Vehicle (PHEV) architectures. Based on the evaluation and selection criteria established, the 2-Mode EVT system with the 1.3 L L4 Diesel Engine showed vastly superior performance in terms of the target VTS, clearly outperformed the alternate architectures in the highest weighted parameters: Fuel Economy, GHGs, PEU and acceleration.

Table 3-7: Candidate architectures final score.

\begin{tabular}{|l|l|l|l|l|}
\hline Specs & Unit & 2-Mode & BAS+RTA & PHEV \\
\hline UDDS FE & MPGge & 38.1 & 29.2 & 31.6 \\
\hline HWFET FE & MPGge & 38.2 & 33.2 & 40.4 \\
\hline Comb FE & MPGge & 38.1 & 30.9 & 35.0 \\
\hline WTW PEU & kWh/km & 0.30 & 0.37 & 0.53 \\
\hline WTW GHG & g/km & 89.5 & 106.9 & 164.5 \\
\hline Emissions & g/miles & 0.5 & 0.5 & - \\
\hline Range & miles & 269 & 222 & 252 \\
\hline Towing & sec & 7.3 & 6.7 & 5.8 \\
\hline 0-60mph & sec & 8.4 & 8.3 & 13.1 \\
\hline 50-70mph & sec & 4.3 & 4.1 & 7.4 \\
\hline Weight & $\mathrm{lbs}$ & 4,363 & 4,307 & 5,005 \\
\hline Cargo & $\mathrm{m}^{3}$ & 0.79 & 0.83 & 0.79 \\
\hline Passengers & - & 5 & 5 & 5 \\
\hline Score & & 89.9 & 66.4 & 70.9 \\
\hline
\end{tabular}


The 2-Mode diesel-electric hybrid vehicle architecture was projected to best meet the VTS and design goals. The selected 2-Mode design features out-performed overall in aspects of fuel economy, environment impacts and performance. The proposed hybrid electric powertrain included a GM 1.3 L SDE L4 DTH family Euro 5 turbo diesel engine, a GM 2-Mode front wheel drive transmission and a high voltage battery pack with four A123 System 25S2P battery modules in series. The HEV powertrain technical specifications are listed in Table 3-8.

Table 3-8: 2-Mode hybrid electric crossover utility vehicle powertrain components specifications.

\begin{tabular}{|l|l|}
\hline & Specifications \\
\hline Diesel Engine & $\begin{array}{l}\text { 1.3 L SDE L4 DTH Family Euro 5 Diesel Engine } \\
70.8 \mathrm{~kW} @ 4,000 \text { rpm, 200 Nm @ 1,750 rpm }\end{array}$ \\
\hline $\begin{array}{l}\text { Exhaust } \\
\text { Aftertreatment }\end{array}$ & OEM catalyzed particulate filter \\
\hline $\begin{array}{l}\text { 2-Mode } \\
\text { Transmission }\end{array}$ & GM Front Wheel Drive 2-Mode EVT X25F, 2 EVTs \\
\hline $\begin{array}{l}\text { High Voltage } \\
\text { Battery }\end{array}$ & $\begin{array}{l}\text { A123 System Lithium-Ion Phosphate Battery Pack } \\
\text { 330 V nominal, 38 Ah, 12.9 kWh }\end{array}$ \\
\hline Hybrid Controller & $\begin{array}{l}\text { Supervisory Control Unit (SCU), MotoTron GCM-0563-048 } \\
\text { Auxiliary Control Module (ACU), MotoTron HCM-0563-048 }\end{array}$ \\
\hline
\end{tabular}




\section{CHAPTER 4}

\section{2-MODE HYBRID ELECTRIC VEHICLE MODEL}

\subsection{Introduction}

Vehicle simulation software provides an easy and low-cost evaluation platform to estimate vehicle fuel economy and performance. The traditional vehicle design process in the automotive industry delays control system delivery for lack of information about the powertrain components while simulation can provide preliminary evaluation of the control system. Simulation can help shorten design time to meet fast market changes and reduce vehicle design and development cost.

Simulations were performed to finalize the hybrid electric vehicle powertrain selection from the candidate hybrid vehicle architectures. After the VTS were set to choose the best hybrid electric vehicle architecture, the simulation was followed to build the hybrid vehicle model in order to estimate the vehicle performance and fuel economy in the Software-In-the-Loop (SIL) testing. Technically a vehicle simulation model is a mathematical representation of the vehicle physical powertrain components. Therefore, the software-in-the-loop vehicle model is capable of providing the simulation results to evaluate and optimize the vehicle control algorithm.

The objective of the hybrid vehicle simulation was to validate the hybrid system control algorithm in order to improve the energy management system and the vehicle performance. For this purpose, detailed dynamic models of the powertrain components 
were not required since the vehicle dynamics itself was slower than the powertrain components. A quasi-static model provides enough powertrain details to evaluate the vehicle technical specifications. In a quasi-static model, the driver is modeled as a PI or proportional-integral controller by comparing the desired speed from a drive cycle and the vehicle actual speed from the vehicle longitudinal dynamic model. The drive cycles are vehicle speed profiles with vehicle desired speed and the road grades. For vehicle powertrain components, either simplified mathematical equations or lookup tables from the bench test results can be used for modeling. Since the powertrain components modeled with quasi-static components are simple, it reduces the computation time, which makes it possible to run the more complicated hybrid electric vehicle model for the purpose of evaluating the hybrid control algorithm.

A hybrid electric vehicle adds complexity to the simulation model. Since the simulation model is a representation of the physical powertrain components, the extra components in the hybrid powertrain need to be modeled in the software. In a hybrid electric vehicle model, new model parts commonly include a high voltage battery/capacitor, power inverter and electric motors.

It is very challenging work to simulate the hybrid electric vehicle to optimize the hybrid control system algorithm in order to improve both fuel economy and performance. First, more powertrain components need to be integrated in the model. Second, an accurate high voltage battery is difficult to model to simulate the complicated chemical reaction process. Finally, a hybrid control system provides much more flexibility in the energy management system to improve fuel economy and performance. 


\subsection{Vehicular Modeling Methods}

\subsubsection{Dynamic Model}

The purpose of a vehicle dynamic model is to study fast response of the powertrain based on mathematical equations of the system. Commonly the dynamic model of the component is expressed using sets of ordinary differential equations (ODE) and partial differential equations (PDE). A dynamics model is applied to simulate fast effects for the optimization control of comfort, stability and drivability. Examples of the dynamic models include engine inlet manifold dynamics, Exhaust Gas Recirculation (EGR) flow rate dynamics and heat transfer in the cylinder wall. The drawback of the dynamic model is that the computation complexity is added to the simulation, especially if the simulation model is large itself. Therefore, dynamic models are uncommon for simulation of a vehicle level to evaluate the slow response activities, such as fuel economy and acceleration time.

Many simulation software offer the dynamic simulation models of vehicle powertrain systems. MATLAB/Simulink is a very basic mathematical tool for any model/simulation. Automotive related sub-systems and components are also available for vehicle powertrain simulation in Simulink. A automotive dynamic simulation software, such as CarSim, AMESim, GT-SUITE, dSPACE and Modelica, are introduced in the following sections.

\subsubsection{Quasi-static Model}

Most of the simulation at the vehicle/system level is to solve the relatively slow response problem, such as vehicle fuel economy and performance. Detailed dynamic specifications of the components are commonly not necessary for the simulation. Hence, 
the quasi-static model is recommended for the simulation at a high level, such as simulation of the whole vehicle to evaluate fuel economy or performance.

There are some popular quasi-static simulation software in the market, such as PSAT and ADVISOR. ETH Zurich developed a free quasi-static simulation toolbox for academic purposes that can be run in any MATLAB/Simulink environment.

\subsubsection{Forward-looking Model}

In a forward-looking model, the vehicle is modeled in reality with the driver's command. A desired vehicle driving schedule is passed to the driver model, which generates the related accelerator or brake pedal command and outputs to the different powertrain and component controllers. In the vehicle speed close-loop system, the driver model modifies its pedal command depending upon how close the speed profile is followed. The components react as in reality to the driver's command, and advanced component features such as dynamic effects can be taken into account, as well as the realistic control strategies that would be implemented in real-time applications. With the forward-looking model, components and configurations can be easily modified, added or removed from the model. This makes it easy to evolve a conventional vehicle model to a hybrid model, which emulates the process of modifying a conventional vehicle to a hybrid vehicle. Figure 4-1 shows the forward-looking model showing the interactive connection between the driving schedule, driver, hybrid controller, powertrain components and vehicle longitudinal model. 


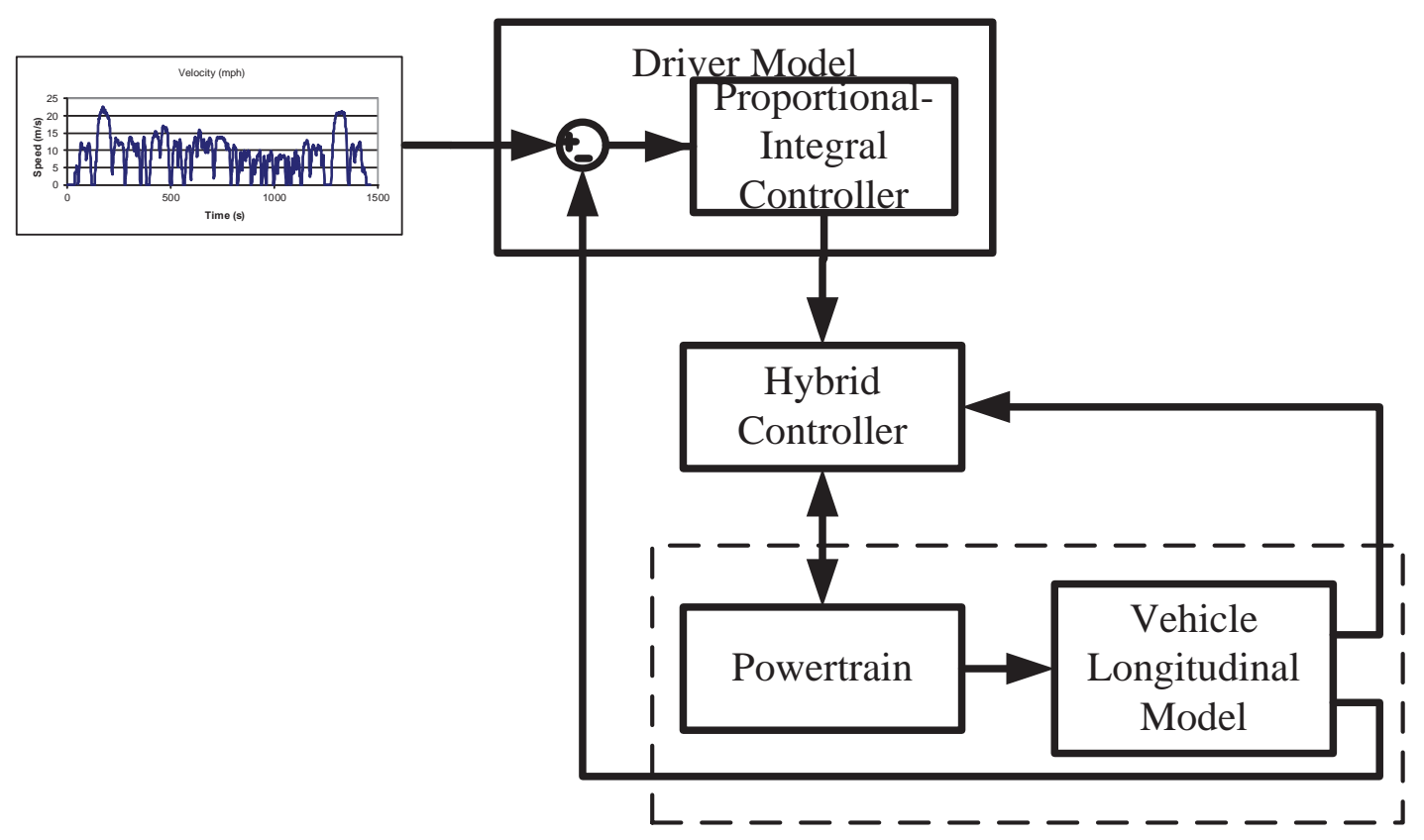

Figure 4-1: Forward-looking model.

\subsubsection{Backward-looking Model}

In a backward-looking model, flow of energy starts from the wheel speed and spreads toward the engine and electric motor in a hybrid electric vehicle. The desired vehicle speed goes from the vehicle longitudinal model back to the engine through the powertrain components, with a series hybrid vehicle example shown in Figure 4-2. During the backward-looking process, each component command will be found to follow the speed cycle. Because of the backward-looking model specification, only a quasi-static model can be used and a realistic control algorithm cannot be developed. 


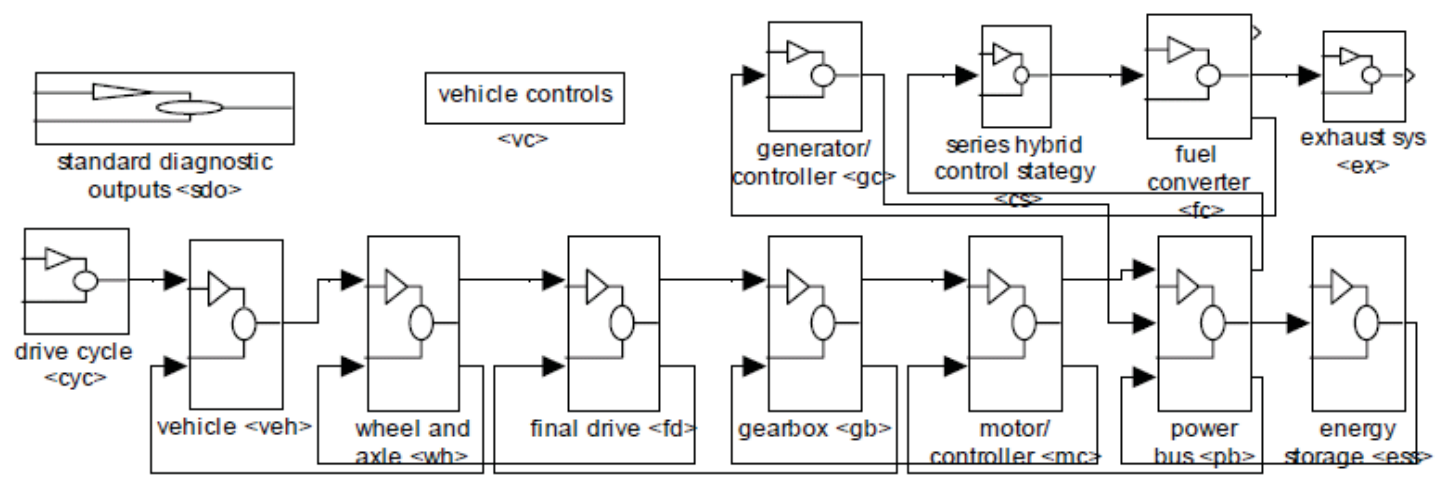

Figure 4-2: Backwards-looking series hybrid model.

\subsection{Vehicle Modeling Tools}

\subsection{1 $\underline{\text { PSAT }}$}

Since 1999, Argonne National Laboratory has been developing a vehicle simulation tool to assess the fuel consumption and performance of advanced vehicles [36]. The software, PSAT, has become widely accepted by industry, universities and research laboratories. PSAT has been used to support research expertise related to advanced vehicle technologies. PSAT is a forward-looking model using Simulink tools for vehicle components and control strategy simulation. For conventional vehicles, the main components of the conventional vehicle are: Vehicle, Wheel, Differential, Gearbox, Torque Converter, Engine, Mechanical and Electrical Accessories, Starter, Generator and Torque Coupling, and Battery. For hybrid electric vehicles, other necessary components are motor/generators, high voltage battery/capacitor, inverter and converters. There are also models for the Driver and the Exhaust Aftertreatment. Various control strategies can be selected to optimize both performance and fuel economy. One of the greatest 
versatilities of PSAT is that parameterized physical models are defined such that a wide variety of component sizes can be considered.

During a simulation, the Driver discretely compares the difference between the schedule speed and the actual speed, and sends a torque demand, based on the vehicle dynamic losses, to the Vehicle Controller. Then, the Vehicle Controller decides how to provide the desired torque demand and sends its commands to the Component Controller which in turn translates the demands into component specific inputs, e.g. (throttle position).

\subsection{2 $\underline{\text { ADVISOR }}$}

ADVISOR, a MATLAB-based modeling tool, enables a user to simulate a conventional, hybrid, electric, or fuel cell vehicle over a city or highway drive cycle and predict the vehicle performance (fuel economy, emissions, battery power, heat generated, and other measurements) [37]. ADVISOR includes vehicle models of engines, batteries, motors, exhaust aftertreatment systems, transmissions, accessory loads, and other characteristics. ADVISOR's quasi-static analysis of component performance means that simulations are fast. Capabilities include component selection and sizing for conventional, hybrid and fuel cell vehicles, energy management strategies, optimization, and target development. The user can change simulation results in fuel economy, performance, or emission by selecting different vehicle component technologies (such as CI vs. SI engine, AC vs. DC motor), sizes, and parameters.

ADVISOR employs a unique combination of backward and forward-facing simulation attributes [38], [39]. A purely backwards facing simulation propagates a high level requirement (e.g. change from $\mathrm{X}$ to $\mathrm{Y}$ speed in $\mathrm{Z}$ seconds) linearly backwards 
through a series of systems (e.g. vehicle-> wheels->transmission->engine). While a forward-facing approach iteratively modifies individual component control commands to the various vehicle subsystems in an effort to find the combination that minimizes the error between the driver demand and the actual response of the system to the control commands. Each has advantages and disadvantages and each excels in their intended applications described in the previous section.

ADVISOR is typically performed to test and analyze vehicle fuel economy and performance in a drive cycle. There are more than 40 different drive cycles that can be chosen in the ADVISOR library. A custom drive cycle developed by the user can be integrated in ADVISOR, including the road grade condition. ADVISOR can test vehicle performance to assess the vehicle powertrain capability during acceleration and hill climbing. Special test procedures are also developed in ADVISOR to test the vehicle in a special drive cycle which combines different standard drive cycles.

There are some limitations while using ADVISOR. ADVISOR component models are quasi-static and should not be used alone to analyze transient characteristics with very small time steps. ADVISOR should not be used to analyze the component's fast dynamic specifications, such as physical vibrations or electric field oscillations.

\subsection{3 $\underline{\text { CarSim }}$}

CarSim simulates the dynamic behavior of racecars, passenger cars, light trucks, and utility vehicles [40]. CarSim animates simulated tests and outputs over 800 calculated variables to plot and analyze, or export to other software such as MATLAB, Excel, and optimization tools. 
CarSim replicates the vehicle system-level behavior with equations of the math models. The equations of the math models describe the basic 3D kinematical and dynamical equations of a vehicle with pneumatic tires and conventional suspension, steering, braking and power systems [41], which contains the major effects of how the tire contacts the road and how the forces from the road are transferred to the chassis through the suspension system. However, details showing the linkage connections and structures are lacking. The CarSim has been widely used in the industry to develop advanced controls, such as predicting vehicle motions related to handling, maintaining directional and roll stability, and promoting braking and acceleration.

The equations of the math models are solved by the special solver program, VehicleSim [42]. The VehicleSim technology has high efficiency, allowing real-time operation for Hardware-In-the-Loop (HIL) simulation, such as dSPACE, ETAS ASCET. The VehicleSim models are extensible by users to add new output variables, controllers and automation options at run time. The VehicleSim models use application program interfaces to connect with nearly any simulation environment [43], such as MATLAB/Simulink, LabVIEW.

\subsection{4 $\quad \underline{\text { AMESim }}$}

Advanced Modeling Environment for performing Simulations of engineering systems (AMESim) is a 1D lumped parameter time domain simulation platform used to analyze fast dynamics in the automotive, aerospace and other advanced manufacturing industries. A literature review indicates that AMESim was used to develop vehicle powertrain models, evaluate vehicle drivability and engine control unit in the HIL environment [44], [45], [46]. 
AMESim uses symbols to represent individual components, which is quite similar to Simulink. However, Simulink components are modeled with purely mathematical equations for automation experts, while AMESim components are interconnected with symbol ports by rules based on the exchange of power through the connections, which obeys the engineering rules [47]. With these symbol connection rules, the AMESim allows the link between different physics domains in mechanical, electrical, electronics, hydraulic, magnetic, pneumatic, thermodynamics and mechatronics.

\subsubsection{GT-Power}

GT-SUITE is a product of Gamma Technologies Inc., which provides simulation tools solely focused on the engine and vehicle industry [48]. GT-SUITE is widely used in the automotive industry and research organizations to model fast dynamic systems in engines and vehicle powertrains [49], [50], [51]. GT-SUITE can execute integrated simulations of the entire vehicle and engine system. GT-SUITE provides versatile multiphysics platform by providing the libraries in flow, acoustics, thermal, mechanical, electric and electromagnetic, chemistry and controls, which are modeled with consideration of multi-body dynamics, kinematics and flexible-body elements.

GT-POWER is part of the GT-SUITE, which features the analysis capability of engines with emphasis on driveline components, valvetrain components, fuel injection and hydraulics, engine heat management and cooling and crankshaft dynamics. GTPower is the industry standard for engine simulations. GT-POWER uses a $1 \mathrm{D}$ dynamics model to represent the flow and heat transfer in piping and related components. GTPOWER consists of two powerful software domains as GT-ISE (Integrated Simulation 
Environment) and GT-POST. GT-ISE develops, executes and manages the simulation of the model, while GT-POST provides plotting tools to access the simulation results.

\subsection{6 $\quad$ dSPACE ASM}

dSPACE has real-time Automotive Simulation Models (ASM) for powertrain and vehicle dynamics. The ASM model consists of open MATLAB/Simulink models with engine, drivetrain, and vehicle dynamics. The model is typically used on the dSPACE simulator for HIL testing of electronic control units or during the design phase of controller algorithms for early validation by offline simulation.

HIL simulation provides the test platform for the control algorithm and communication of the electronic control units in a real-time environment. The real-time environment provided is built on the high speed processor board and multiple I/O boards that sense or measure the digital signal, analogue signal, PWM signal, hall sensor signal, current signal and other special signals. A schematic view of the HIL system with ASM model is shown in Figure 4-3 [52]. The ASM Simulink model of engine or vehicle dynamics is run on the real-time simulator, which measures the signals from the model and feeds back to the electronic control units with electrical signals. The electronic control unit operates as if it were in a vehicle by reading the engine/vehicle status and generating the related control commands, which will be measured by the simulator and fed back to the model. So the electronic control unit's control algorithm is tested on a real-time hardware environment. 


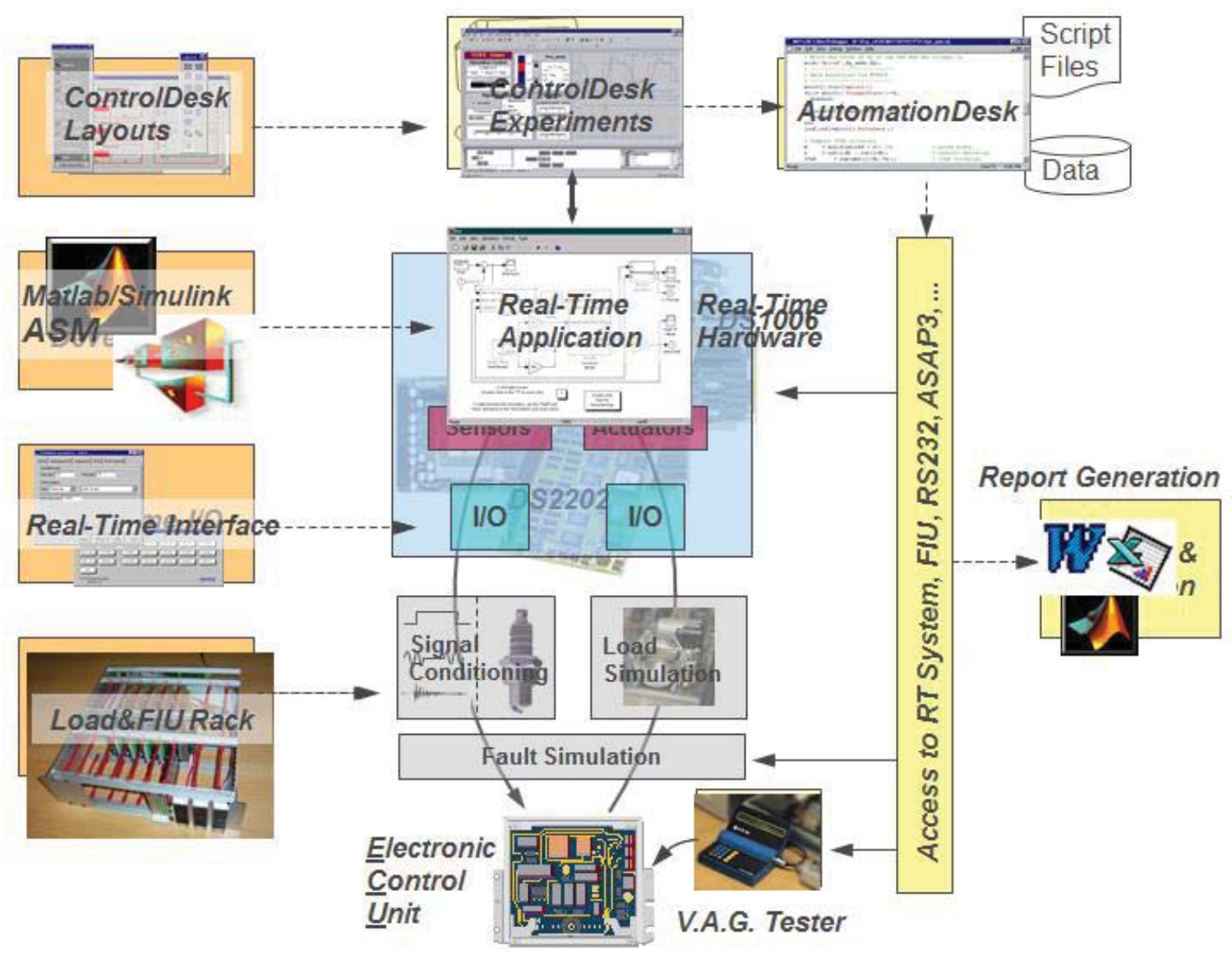

Figure 4-3: HIL simulation system [52].

\subsection{7 $\quad \underline{\text { Modelica }}$}

Modelica models dynamics behavior of engineering systems through multidomains including mechanical, electrical, thermal, hydraulic, pneumatic, fluid and control [53]. Modelica models are described by differential, algebraic and discrete equations, but not by partial differential equations as in finite element method and computational fluid dynamics.

Modelica uses object-oriented, non-causal language for modeling of large systems. Modelica models physical components from class-represented subsystems. The Modelica has the benefits of the object-oriented programming language in re-usability, 
hierarchy and inheritance. A large area of the physical process can be made by connecting classes in a small system level. In a vehicle model, the car is a class containing the engine which includes the classes of cooler, valve and cylinder.

Modelica provides a free vehicle dynamics library with 3D mechanics and a drivetrain library with 1D mechanics to model the dynamics of passenger cars and trucks. Modelica lets the user build a custom library of classes by using a number of standard libraries. A large library of components is available in chassis, suspensions, tires, steering, driveline and brakes. An advanced commercial vehicle dynamics library is available from Modelon [54], but is no longer free.

\subsection{2-Mode Hybrid Electric Vehicle Modeling}

\subsubsection{2-Mode Hybrid Powertrain Schematics}

The designated 2-Mode hybrid powertrain included a GM 1.3 L diesel engine, a GM X25F 2-Mode transmission and an A123 System 330 V 39.2 Ah/12.8 kWh high voltage battery pack, shown in Figure 4-4. The Transmission Powertrain Inverter Module (TPIM) was the transmission power inverter module, including a Hybrid Control Process (HCP) and the motor control modules. The high voltage battery was managed by the OEM Battery Management System (BMS), including the function of controlling the main relays and feeding back the battery status.

The GM X25F was a new type of 2-Mode hybrid transmission developed for FWD crossover utility vehicles. It was originally designed by GM for use in the 2009 Saturn Vue Hybrid. The X25F consisted of 2 planetary gear sets, 4 wet-plate clutches for gear selection, 1 wet-plate damper bypass clutch for vehicle starting and 2 electric motor/generators [55]. Transmission gear selection was achieved by engaging or 
disengaging four wet-plate clutches: C13, CB12R, C2324 and CB4. Clutch switching and torque application were controlled by the Transmission Control Module (TCM).

Motor/Generator A and Motor/Generator B were Permanent Magnet Synchronous

Motors (PMSM) to achieve the electrically variably transmission function by continuously controlling the transmission ratio.

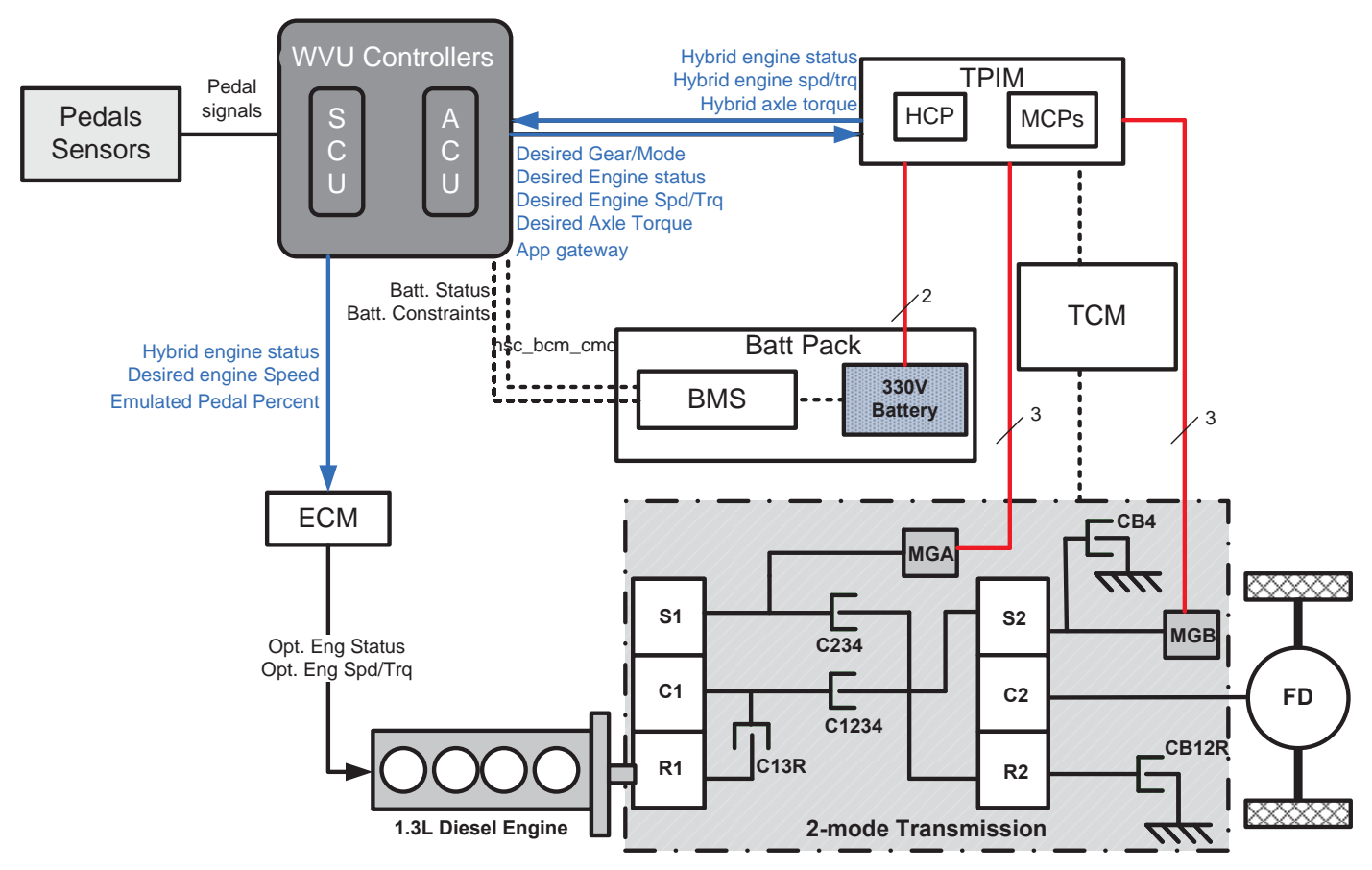

Figure 4-4: 2-Mode hybrid powertrain schematics.

\subsection{2 $\quad$ High Level Schematics}

The 2-Mode hybrid electric vehicle powertrain described above was simulated as a quasi-static model, shown in Figure 4-5 in detail. The driver pedal position was output to the hybrid system controller, which applied the control algorithm to distribute the power request between the engine and the electric motors. The hybrid control algorithm emulated the hybrid function of the SCU in Figure 4-4. 
The hybrid system controller algorithm is described in details in CHAPTER 8, while this chapter continues with the plant model of the simulation.

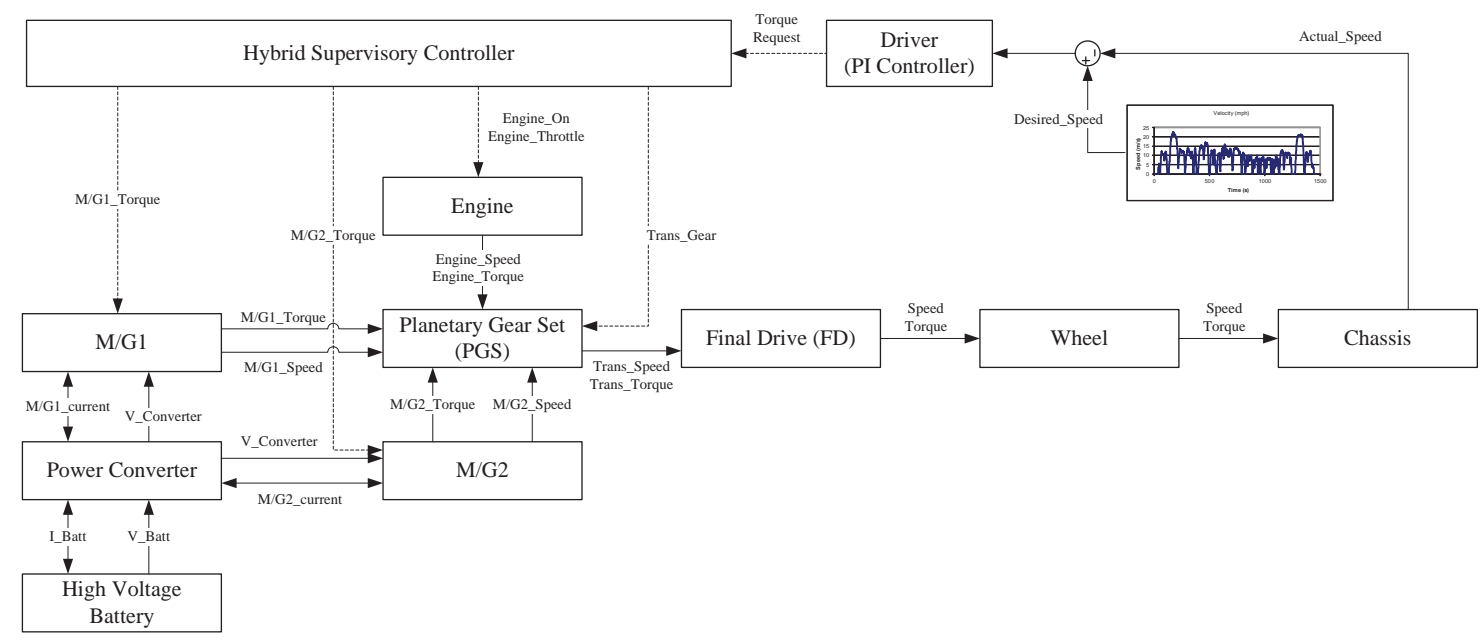

Figure 4-5: 2-Mode hybrid electric vehicle model architecture.

\subsection{3 $\quad \underline{\text { Driver Model }}$}

In the forward-looking model, the driver model imitates a human driver's command of generating accelerator and braking pedal commands. The model of the driver was accomplished with the difference of the desired and actual vehicle speeds and the acceleration or brake pedal was commanded using to a PI controller, shown in Eq. 4-1 and Eq. 4-2. A proportional-integral-differential controller is sometimes used to consider the differential part of the speed error [56].

$$
\begin{gathered}
T_{c m d}=k_{p}\left(v_{\text {veh_actual }}-v_{\text {veh_desired }}\right)+k_{i} \cdot \int\left(v_{\text {veh_actual }}-v_{\text {veh_desired }}\right) d t \quad \text { Eq. 4-1 } \\
T_{\text {loss }}=\left(m_{\text {veh }} \cdot \frac{d v}{d t}+0.5 \cdot \rho \cdot C_{d} \cdot A \cdot v^{2}\right) \cdot R_{\text {wheel }}+c_{r r} \cdot m_{\text {veh }} \cdot g \cdot R_{\text {wheel }}
\end{gathered}
$$


Where: $T_{c m d}$ is vehicle torque command; $k_{p}$ is proportional control parameter; $k_{i}$ is integral control parameter; $v_{v e h \_a c t u a l}$ is actual vehicle speed; $v_{v e h \_d e s i r e d}$ is desired vehicle speed; $T_{\text {loss }}$ is vehicle torque loss; $R_{\text {wheel }}$ is wheel radius.

$$
\left(m_{v e h} \cdot \frac{d v}{d t}+0.5 \cdot \rho \cdot C_{d} \cdot A \cdot v^{2}\right) \cdot R_{\text {wheel }} \text { was modeled in the vehicle dynamics, }
$$

and $c_{r r} \cdot m_{v e h} \cdot g \cdot R_{\text {wheel }}$ was modeled in the vehicle wheel.

\subsubsection{Engine Model}

\subsubsection{Engine Model Review}

Different methods are applied to model IC engines. IC engines can be simulated using a dynamic model or quasi-static model. The difference and application of dynamic models and quasi-static models were discussed in previous section. In a quasi-static model, the engine can be modeled using different methods, such as Willan's Line Model, mean value model, or engine map from dynamometer test data.

The Willan's Line Model uses a scaling technique to create a quasi-static model of IC engines independent of engine geometric sizes [57]. The Willan's Line Model was originally applied to describe the linear relationship between the brake mean effective pressure and fuel consumption to estimate the engine friction losses [57]. The advantage of this method is that the model can be used to represent a series of engines. However, the details of the engine are not modeled, such as fuel injectors, thermal effects, valve dynamics, etc. Details on the Willan's Line model can be found with the reference [58], [59], [60]. The Willan's Line Model generates the engine efficiency and fueling map based on the known engine test data in the same category. However, the Willan's Line Model doesn't guarantee that the fuel map and the efficiency map are accurate. In Willan's Line Model, the basic two parameters describing the engine are mean piston 
speed in $\mathrm{m} / \mathrm{s}$ and mean effective pressure in $\mathrm{N} / \mathrm{m}^{2}$, instead of engine speed in rpm and engine torque in Nm. The detailed process and equations of building the Willan's Line Model can be found in [61].

The Mean Value Model (MVM) has been widely and successfully implemented to model IC engines [62], [63], [64], [65], [66]. A typical MVM diesel engine model can be seen in Figure 4-6. The air path simulates the compressor, the intercooler, the throttle, and the intake manifold dynamics on the intake side, and the turbine, the EGR with cooler, and the exhaust manifold on the exhaust side.



Figure 4-6: Typical MVM diesel engine model [67].

The fuel system model can include a common-rail system and a unit injector. The common-rail system consists of several modules like the high pressure pump, fuel metering unit, pressure control valve and injector. The model for the unit injector system is a map-based approach for the injector itself. 
The cooler block calculates the cooling water temperature from the indicated mean torque and the friction torque. It is assumed that the friction torque is completely dissipated to the cooling circuit and also that a fixed amount of the indicated mean torque is dissipated by the cooling system. This part can be seen as the wall heat losses from the cylinder to the cooling circuit.

The engine combustion block describes the air flow through the inlet valve and the torque generated by the combustion process. The effective torque consists of the mean indicated torque and the friction torque. The effects of the injection angle and of the air/fuel ratio are included as two efficiencies. The combustion torque is modeled as a mean value. To simulate the individual cylinder torques, a crank angle based function is multiplied by the mean indicated torque.

\subsubsection{2 $\quad$ Research Engine Model}

The engine model was simulated with the bench experiment data provided by GM. The engine was modeled in hot condition only to estimate the torque and fueling rate as the engine dynamometer test data was available to create the model with acceptable accuracy. The engine efficiency map and the fueling rate map were modeled with the dynamometer test data as a function of the engine's brake torque and engine speed. In the engine model, the engine torque was commanded by the Diesel Engine Control Module (DECM). The engine torque command was filtered with the engine lug curve, shown in Figure 4-7, and engine stop-start status. If engine was off, the engine torque command was set to zero. The engine torque command was limited under the maximum torque defined by the lug curve as a function of engine speed. The control logic was shown in Figure 4-8, where $T_{\text {eng_cmd }}$ is engine torque command; $\omega_{\text {eng }}$ is engine 
speed; $T_{\text {out }}$ is engine output torque; $T_{\text {wor }}$ is engine maximum torque with wide open throttle.



Figure 4-7: 1.3 L Diesel engine power and torque lug curve.

$$
\begin{aligned}
& \text { Input of engine speed } \omega_{\text {eng }} \text { and engine torque } T_{\text {eng_cmd }} \\
& \text { IF } \mathrm{T}_{\text {eng_cmd }} \geq 0 \text { and } \omega_{\text {eng }}>0 \\
& \mathrm{~T}_{\text {out }}=\left(1-\mathrm{T}_{\text {eng_cmd }}\right) \cdot \mathrm{T}_{\text {CTT }}+\mathrm{T}_{\text {eng_cmd }} \cdot \mathrm{T}_{\text {WOT }} \\
& \text { ELSE IF } \mathrm{T}_{\text {eng_cmd }}=0 \text { and } \omega_{\text {eng }}=0 \\
& \mathrm{~T}_{\text {out }}=0 \\
& \text { END IF }
\end{aligned}
$$

Figure 4-8: Engine torque control logic.

Once the engine torque and speed required to drive the designated cycle was decided, the fuel consumption was estimated to calculate fuel economy. With the quasistatic method, the fuel consumption to sustain the requested speed and torque was directly mapped to the torque-speed plane. The engine fueling rate based on the engine speed and torque command was determined based on the engine test results on a dynamometer, shown in Figure 4-9. 


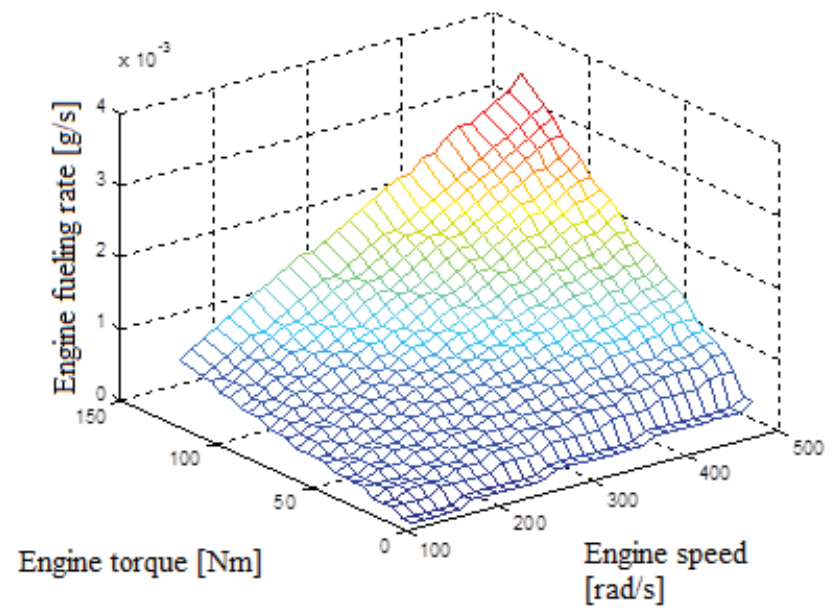

Figure 4-9: $1.3 \mathrm{~L}$ diesel engine fueling map.

The engine fueling rate was specially handled when the vehicle is idling. The engine fueling control method under the idling and low speed was different from the same map mentioned above. If the same method was used, the consequent small measurement with uncertainties could cause substantial errors in the estimation of the fuel economy in the simulation. When the engine torque command was very small, a minimum torque plus the friction torque was commanded. When the engine was shut down, the fueling rate was zero since the hybrid vehicle has the stop-start function. The engine fueling calculation logic is shown in Figure 4-10, where $T_{\text {min_map }}$ is engine friction torque; $\mathrm{T}_{\mathrm{C} \text { Tा }}$ is close-throttle torque, the engine drag torque when no fuel flows into the engine; $\dot{m}_{\text {fuel }}$ is instantaneous fueling rate; $m_{\text {fuel }}$ is fueling rate. 


\begin{tabular}{|c|c|}
\hline Input of engine speed $\omega_{\text {eng }}$ and engine torque $T_{\text {eng }}$ \\
\hline Yes & Yes \\
\cline { 2 - 2 }$\dot{m}_{\text {fuel }}=f\left(\omega_{\text {eng }}, T_{\text {eng }}\right)$ & $\dot{m}_{\text {fuel }}=\frac{\mathrm{T}_{\text {eng }}-\mathrm{T}_{\text {CTT }}}{\mathrm{R}_{\text {min_map }}-\mathrm{T}_{\text {CTT }}} \dot{m}_{\text {fuel }} \mid \dot{m}_{\text {fuel }}=0$ \\
\hline$m_{\text {fuel }}=\int \dot{m}_{\text {fuel }} d t$ \\
\hline
\end{tabular}

Figure 4-10: $1.3 \mathrm{~L}$ diesel engine fueling rate calculation control logic.

\subsubsection{Electric Motor Model}

\subsubsection{Electric Motor Model Review}

The AC motor is more often used in hybrid electric vehicles for high power density compared to a DC motor. Among AC motor categories, PMSM is more popular in HEV for providing higher power density for their size compared to AC inductance motors. In an AC induction motor, in order to produce rotor flux, part of the stator current is used to induce rotor current, which generates extra heat in the motor. The PMSM contains permanent magnets as the stator, while the rotors are three-phase windings. This special architecture builds a cross bridge between an ACIM and a brushless DC motor.

An AC motor can be simulated with the electric-magnetic rules of electric motors. In the PMSM, the d-q frame is commonly used to simplify the motor model. In the d-q frame, the inductance parameters become constant, independent of motor shaft position. Because the d-q frame utilizes space vectors, the model of the PMSM provides a powerful physical interpretation of the interactions taking place in the production of 
voltages and torques, and, more importantly, it leads to the ready adaptation of positionalor speed-control strategies such as vector control and direct torque control.

Similar to the Willan's model used in the IC engine, the Willan's model is also used to model EM in terms of the efficiency. The Willan's model is scalable for the same category, such as AC Induction Motor (ACIM) and PMSM. The Willan's model takes the form of equation Eq. 4-3:

$$
P_{\text {out }}=\eta \cdot P_{\text {in }}-P_{\text {other }} \text { loss }
$$

Where: $P_{\text {out }}$ is the output power or mechanical power; $P_{\text {in }}$ is the input power or electric power; $\eta$ is the electric machine indicated efficiency; $P_{\text {other_loss }}$ is the power losses due to friction, heat losses, etc.

Two normalized variables of the mean rotor speed $c_{m}(t)$ and the electric mean effective pressure are applied as shown in Eq. 4-4 and Eq. 4-5:

$$
c_{m}(t)=\omega(t) \cdot r
$$

Where: $\omega(t)$ is motor mean tangential speed; $r$ is rotor radius.

$$
\begin{aligned}
& \text { electric mean effective pressure } \\
& \qquad=\frac{\text { mean tangential speed force }}{\text { motor external surface }}
\end{aligned}
$$

Where: $c_{m}(t)$ is the mean rotor speed; $\omega(t)$ is the motor speed; $r$ is the rotor radius.

In terms of electric mean effective pressure $P_{m e}(t)$, the scalable Willan's model of an electric motor can be written as Eq. 4-6:

$$
P_{m e}(t)=e \cdot P_{m a}(t)-P_{m r}(t)
$$

Where $e$ is the indicated efficiency. 
The available mean effective pressure $P_{m a}(t)$, and mean loss pressure $P_{m r}(t)$, are defined as Eq. 4-7 and Eq. 4-8:

$$
\begin{aligned}
& P_{m a}(t)=\frac{P_{\text {in }}(t)}{2 \cdot V_{r} \cdot \omega(t)} \\
& P_{m r}(t)=\frac{P_{\text {loss }}}{2 \cdot V_{r} \cdot \omega(t)}
\end{aligned}
$$

Where: $P_{\text {in }}$ is input power; $V_{r}$ is the rotor volume; $P_{\text {loss }}$ is power loss.

\subsubsection{2 $\quad \underline{\text { Research Electric Motor Model }}$}

An efficiency map based electric motor model was used in the hybrid vehicle model for the convenience that the bench test data was available. The electric motor lookup table represented the static empirical efficiency. The model also included the constant torque and the peak torque map as a function of the electric motor speed. The model included three essential maps:

- Continuous torque as a function of speed

- $\quad 10$-second maximum torque as a function of speed

- Four-quadrant efficiency map as a function of speed and torque.

The motor torque map is shown in Figure 4-11, and the motor efficiency contour map in first quarter is shown in Figure 4-12. The electric motor efficiencies in the other three quarters were mirrored from the map in the first quarter. 


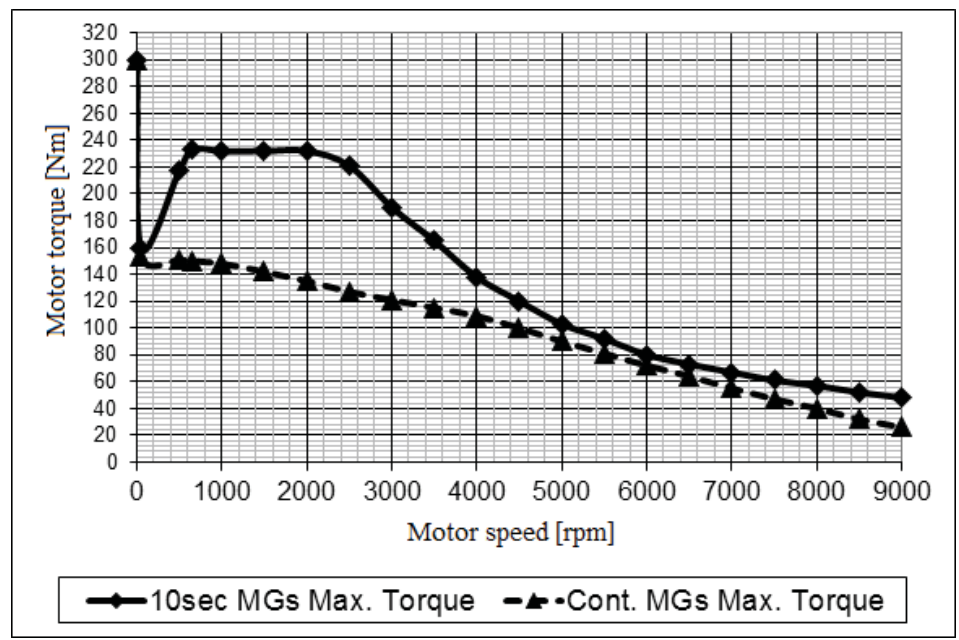

Figure 4-11: 2-Mode electric motor continuous torque and 10-second maximum torque.

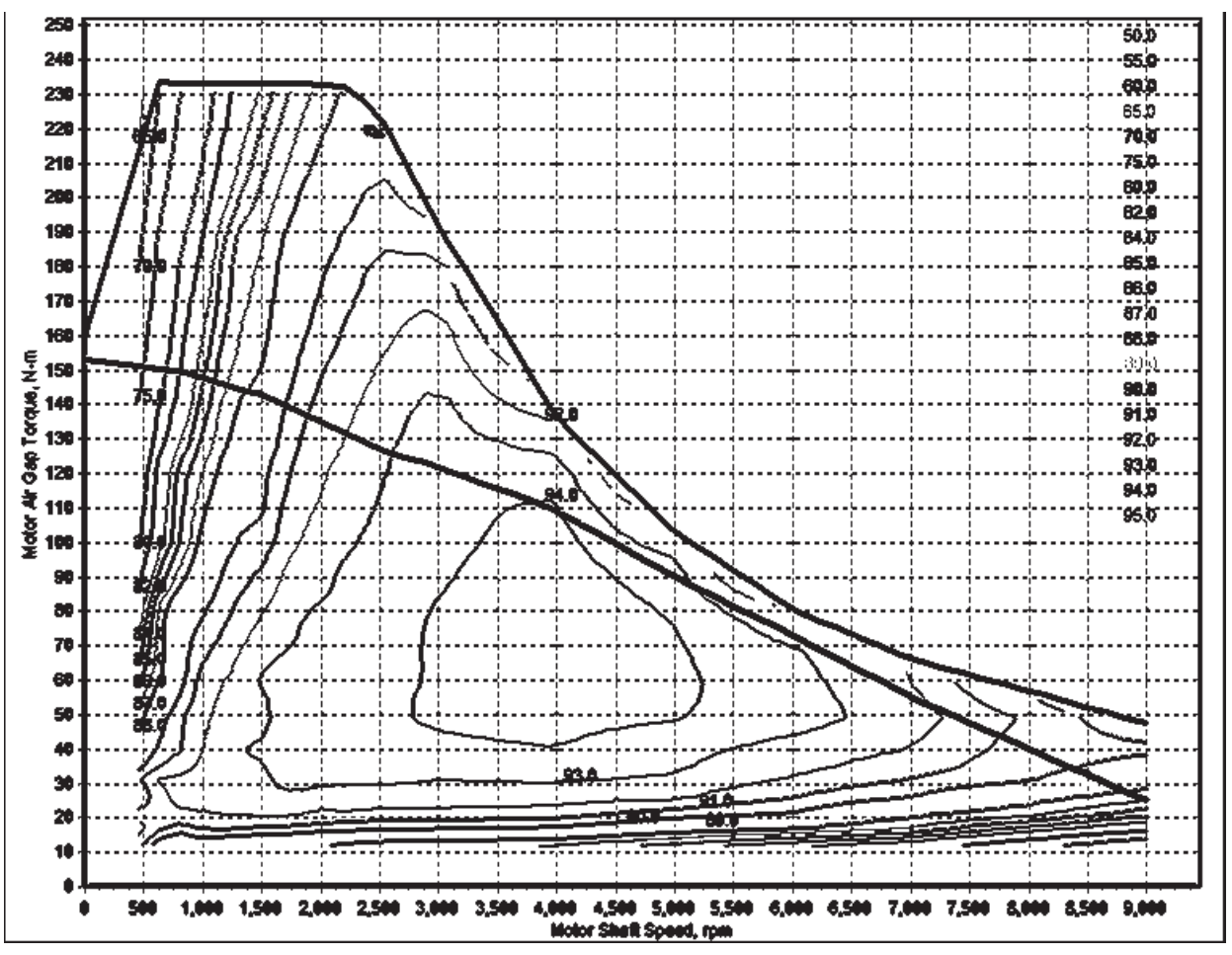

Figure 4-12: 2-Mode electric motor efficiency map.

A heat dissipation index was used to interpolate the electric motor instantaneous available torque between the motor continuous torque and the motor short-time 
maximum torque, shown as Eq. 4-9. Then the maximum available torque was limited by Eq. 4-10. There was no dependence of the maximum torque curve or efficiency map on the motor input voltage.

$$
\begin{gathered}
H I=-0.3+\int \frac{0.3}{\tau}\left(\frac{T_{\text {out }}}{T_{\text {cont }}}-1\right) d t \\
T_{\text {avaible }}=T_{\text {peak }}-\left(T_{\text {peak }}-T_{\text {cont }}\right) \cdot H I
\end{gathered}
$$

Where: HI is heat index; $T_{\text {out }}$ is motor output torque; $T_{\text {cont }}$ is motor continuous maximum torque; $T_{\text {avaible }}$ is motor instantaneous available torque; $T_{\text {peak }}$ is motor 10 second peak torque.

\subsection{6 $\quad$ Battery Model}

\subsubsection{Battery Model Review}

A battery can be modeled as complicated as considering the effect of internal resistance, internal inductance, double layer and diffusion effects of capacitor and resistance, shown in Figure 4-13. The battery cell model calculates the terminal voltage, the battery SOC and the battery temperature as functions of the charge and discharge current [68].

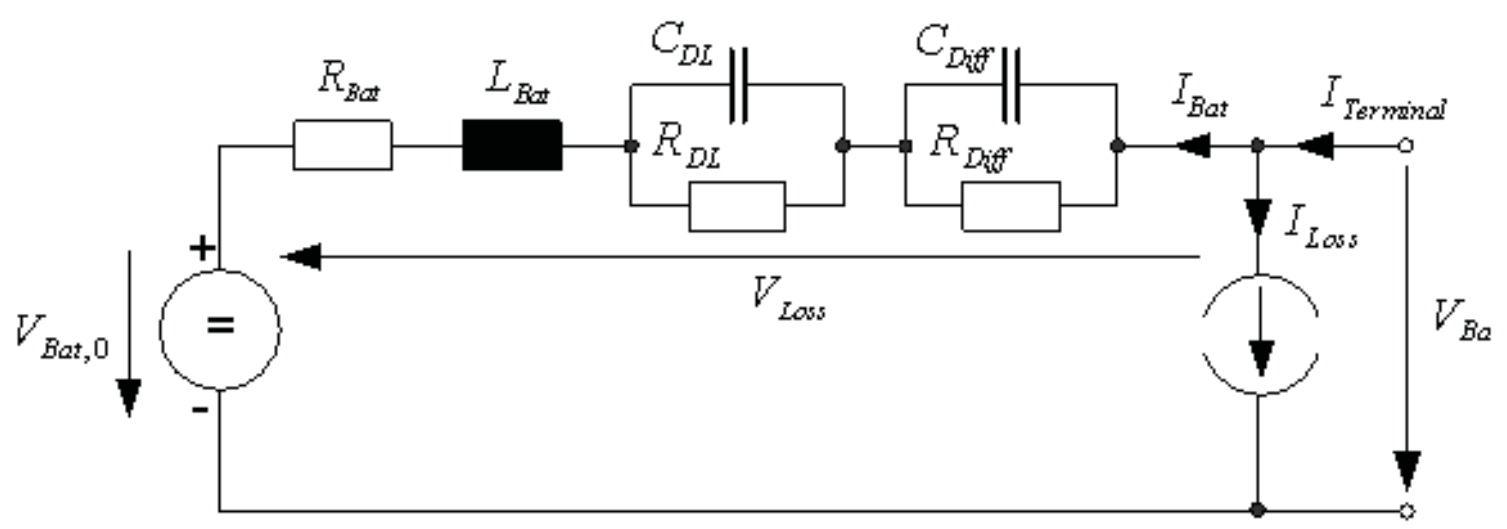

Figure 4-13: dSPACE battery model. 
The battery terminal voltage is calculated with Eq. 4-11:

$$
V_{\text {batt }}=V_{\text {Batt }, 0}+V_{\text {loss }}
$$

Where: $V_{\text {batt }}$ is battery voltage; $V_{B a t t, 0}$ is battery nominal voltage; $V_{\text {loss }}$ is battery loss voltage.

The initial battery voltage is the sum of the individual cell voltages according to the state of charge, shown in Eq. 4-12:

$$
V_{\text {batt }, 0}=n_{\text {cell }} \cdot f_{v_{-} \text {cell }}(S O C)
$$

Where: $n_{\text {cell }}$ is the number of cells; $f_{v_{-} \text {cell }}$ is the cell voltage as a function of the cell SOC.

The battery loss voltage according to the charge and discharge current effect on internal resistance, internal inductance, capacitance and resistance of double layer and diffusion effects, shown in Eq. 4-13 [68]:

$$
\begin{gathered}
V_{\text {loss }}(t)=\frac{1}{C_{D L}} \int\left(I_{\text {batt }}-\frac{V_{D L}}{R_{D L}}\right) d t+\frac{1}{C_{\text {diff }}} \int\left(I_{\text {batt }}-\frac{V_{\text {diff }}}{R_{\text {diff }}}\right) d t \\
+I_{\text {batt }} f_{\text {batt }}\left(\text { SoC }, I_{\text {batt }}, T_{\text {batt }}\right)+\frac{d I_{\text {batt }}}{d t} L_{\text {batt }}
\end{gathered}
$$

Where: $\mathrm{V}_{\text {batt }}$ is battery terminal voltage; $\mathrm{V}_{\text {batt, } 0}$ is balanced voltage; $\mathrm{V}_{\text {loss }}$ is loss voltage; $V_{\text {Cell }}$ is cell voltage; $V_{D L}$ is double layer loss voltage; $V_{\text {diff }}$ is diffusion loss voltage; $\mathrm{C}_{\mathrm{DL}}$ is double layer capacitance; $\mathrm{C}_{\text {diff }}$ is diffusion capacitance; $\mathrm{R}_{\mathrm{DL}}$ is double layer resistance; $\mathrm{R}_{\text {diff }}$ is diffusion resistance; $\mathrm{R}_{\text {batt }}$ is battery resistance; $\mathrm{L}_{\text {batt }}$ is battery inductance; $\mathrm{n}_{\text {cell }}$ is number of battery cells; $\mathrm{T}_{\text {batt }}$ is battery temperature; $\mathrm{I}_{\text {batt }}$ is battery current; SOC is state of charge.

The state of charge is calculated according to the loss current from the thermal model I_loss and battery charge or discharge current, shown in Eq. 4-14: 


$$
S O C=S O C_{\text {ini }}+\frac{1}{k_{N}} \int\left(I_{\text {Terminal }}-I_{\text {Loss }}\right) d t
$$

Where: SOC is state of charge; $\mathrm{SOC}_{\text {ini }}$ is initial state of charge; $\mathrm{K}_{\mathrm{N}}$ is Nominal capacity of battery; $\mathrm{I}_{\text {Terminal }}$ is terminal current; $\mathrm{I}_{\text {Loss }}$ is loss current.

In the FreedomCAR program at Argonne National Laboratory, a Li-ion battery model was validated based on the battery cell bench test data, which included cell capacity, cell voltage, cell power, etc. The test data including the impedance of the experimental cell was modeled as Figure 4-14 [69] and the resistance was calculated as Eq. 4-15 [69].

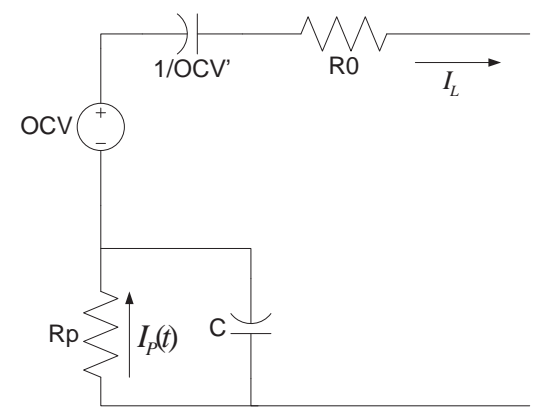

Figure 4-14: Impedance model for a Li-ion battery [69].

$$
\frac{\left(O C V-V_{L}\right)}{I_{L}}=R=R_{0}+\frac{R_{p} I_{p}}{I_{L}}
$$

Where: OCV is open circuit voltage (V); $\mathrm{V}_{\mathrm{L}}$ is cell voltage (V); $\mathrm{R}$ is total cell impedance (milliohms); $\mathrm{R}_{\mathrm{o}}$ is cell internal resistance (milliohms); $\mathrm{R}_{\mathrm{p}}$ is internal polarization resistance (milliohms); $I_{L}$ is cell load current (A); $I_{p}$ is current through polarization resistance (A).

The value for $I_{p}$ is derived by integration of the differential Eq. 4-16 [69]:

$$
\frac{d I_{p}}{d_{t}}=\frac{I_{L}-I_{p}}{\tau}
$$


Where: $\tau$ is chosen or calibrated in the model to fit between the measured voltage and estimated voltage.

The $\mathrm{I}_{\mathrm{p}}$ was solved as Eq. 4-17 [69]:

$$
\begin{gathered}
I_{P}=\left(1-\frac{1-e^{-\Delta t / \tau}}{\Delta t / \tau}\right) I_{L, i}+\left(\frac{1-e^{-\Delta t / \tau}}{\Delta t / \tau}-e^{-\Delta t / \tau}\right) I_{L, i-1} \\
+\left(-e^{-\Delta t / \tau}\right) I_{P_{-}(i-1)}
\end{gathered}
$$

Where: $I_{L, i}$ is (i)th $I_{L}$ sample; : $I_{L, i-1}$ is (i-1)th $I_{L}$ sample; $I_{P_{-}(i-1)}$ is (i-1)th $I_{p}$ sample; $\Delta t$ is sample delta time.

Peukert's equation is widely used to model the battery using battery capacity (Q), discharge current (I), and time (t) [70]. Considering the constant current discharge method, the battery is discharged from full capacity down to the battery cut-off voltage. The load resistance $\mathrm{R}_{\mathrm{L}}$ is varied to change the discharge current level and to maintain the constant current in the same discharge current level. The Peukert's is shown in Eq. 4-18 [70] as:

$$
I^{n} \cdot t_{c u t}=\lambda
$$

Where: $\mathrm{I}$ is constant discharge current in $\mathrm{A}$; $\mathrm{n}$ is a curve-fitting constant, with $\mathrm{n}=1$ for small current and $\mathrm{n}=2$ for large current; $t_{\text {cut }}$ is the actual time to discharge the battery in $\mathrm{h} ; \lambda$ is the capacity at the I discharge rate in Ah.

Peukert's equation defines battery nominal capacity when the battery is discharged at 1 A, shown as Eq. 4-19 [69]:

$$
I \cdot t_{c u t}=Q
$$

Where $\mathrm{Q}$ is the battery capacity at a one-ampere discharge rate. 
Peukert's equation can be used to develop fractional depletion models. The SOC of a battery indicates the current battery capacity. It SOC is defined in Eq. 4-20 [70]:

$$
\operatorname{SoC}(\%)=\frac{Q-\int_{0}^{t} i(\tau) d \tau}{Q} \cdot 100 \%
$$

Where $i(\tau)$ is instantaneous current flow in A.

\subsubsection{2 $\quad$ Research Battery Model}

A simplified model was used in this research as the research was focused on the behavior of the battery in the hybrid vehicle system, not the battery itself. The equivalent circuit of the battery pack included an ideal open-circuit voltage source in series with an internal resistance, shown in Figure 4-15. The battery capacity was taken as constant without considering the effects of temperature and life cycle. The battery was subject to a minimum voltage limit, below which the battery power would shut off.

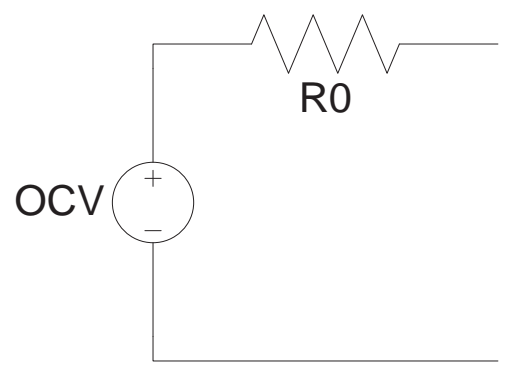

Figure 4-15: Simple battery model.

Battery pack OCV was the cell voltage times the cell number in series. And the cell voltage was calculated as the nominal voltage times the cell voltage factor as a function of the state of charge, simulated as a 1-D look-up table by using the cell hybrid pulse power characterization test data, shown in Figure 4-16. 


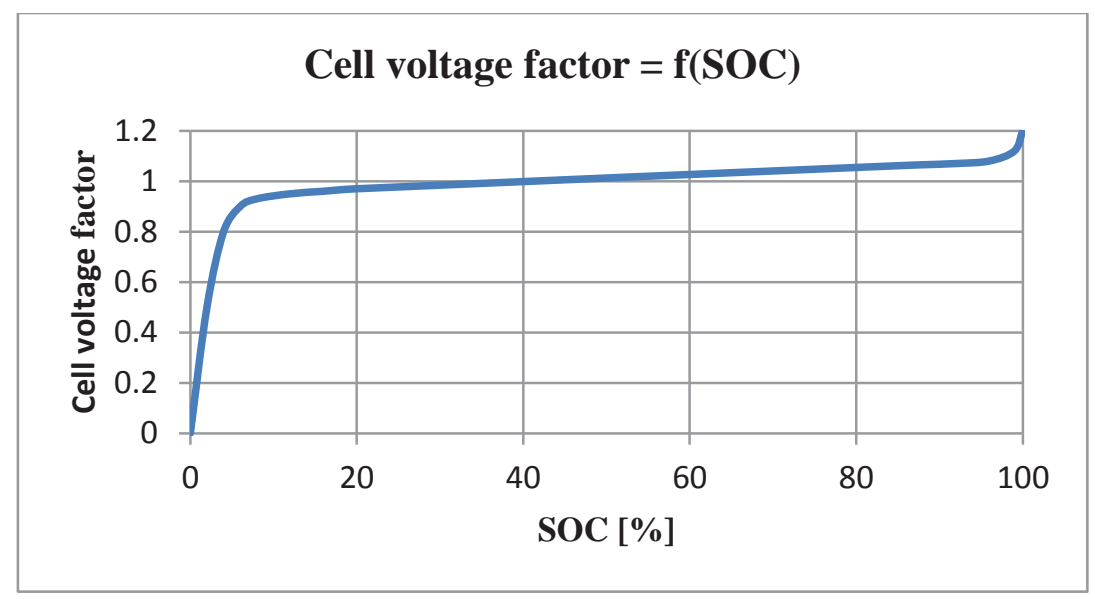

Figure 4-16: Cell voltage factor [71].

Battery cell resistance was modeled with a look-up table using the hybrid pulse power characterization test results. Cell voltage was measured with different discharging current rate in terms of battery SOC during the hybrid pulse power characterization test, shown in Figure 9-20. The charge and discharge current of the battery was measured in C-rate. $1 \mathrm{C}$ rate means that a current charge or discharge in one hour equal to the battery rated capacity. $0.5 \mathrm{C}$ rate means that a current charge or discharge in two hours equal to the battery rated capacity. The battery cell voltage was calculated with Eq. 4-21.

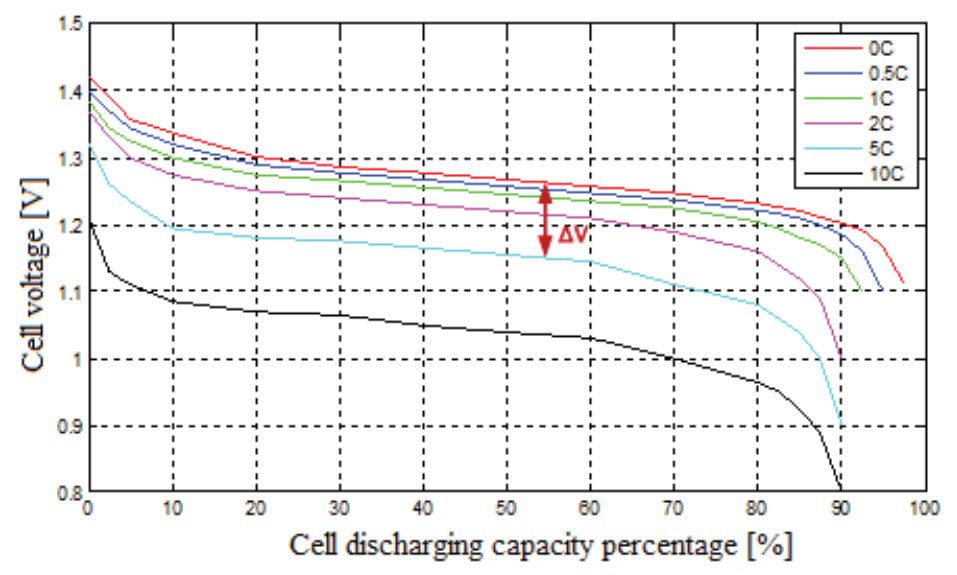

Figure 4-17: Battery cell voltage as a function of SOC and current [71]. 


$$
R_{0}=\frac{\Delta V(S O C)}{I}
$$

Where: $R_{0}$ is internal resistance; $\Delta V(S O C)$ is the change in voltage as a function of SOC; I is battery current.

\subsubsection{Final Drive Model}

A final drive with speed reduction ratio was integrated within the 2-Mode transmission. The final drive was modeled with speed reduction and torque increase. In the torque increase calculation, the torque loss was a 2-D lookup table output based on the transmission speed and torque.

The final drive speed output was calculated with the Eq. 4-22:

$$
\omega_{\text {out }}=\omega_{\text {in }} \cdot r_{f d}
$$

Where $\omega_{\text {out }}$ is final drive output speed, $\omega_{\text {in }}$ is final drive input speed (transmission output speed) and $r_{f d}$ is the final drive gear ratio.

The final drive torque output was calculated with the Eq. 4-23:

$$
T_{\text {out }}=\left(T_{\text {in }}-T_{\text {loss }}\right) r_{f d}
$$

Where $T_{\text {out }}$ is final drive output torque and $T_{\text {in }}$ is final drive input torque.

\subsection{8 $\quad$ Power Inverter Model}

The power converter was modeled as a DC/DC converter, instead of a DC/AC inverter since the electric motor used a quasi-static model with experiment data, which doesn't require modeling of the AC circuit. The DC/DC converter model used a fixed efficiency $\eta$. The DC/DC converter can increase or decrease output voltage. The input voltage and current are from the battery and the output voltage and current are to the electric machines, shown in Figure 4-18. 


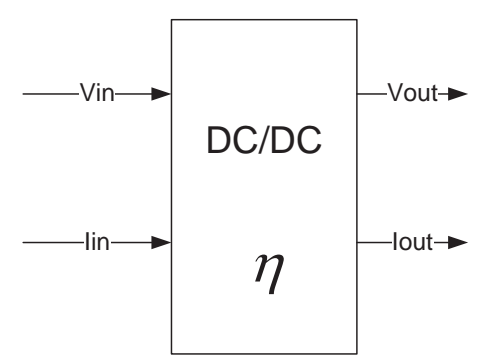

Figure 4-18: DC/DC power converter model.

The current can flow from/into both battery and electric machines, which represents the discharge/charge of the battery and motoring/generating of the electric machines. Under the situation of discharging the battery and motoring electric machine, the model calculated the output current flow, shown in Eq. 4-24:

$$
I_{\text {out }}=\frac{V_{\text {in }} I_{\text {in }} \eta}{V_{\text {out }}}
$$

Where: $I_{\text {out }}$ is output current; $V_{\text {in }}$ is input voltage; $I_{\text {in }}$ is input current; $\eta$ is converter efficiency; $V_{\text {out }}$ is output voltage.

Under the situation of charging the battery and the electric machine generating power, the model calculated the input current flow, shown in Eq. 4-25:

$$
I_{\text {in }}=\frac{V_{\text {out }} I_{\text {out }} / \eta}{V_{\text {in }}}
$$

\subsection{9 $\quad \underline{\text { Vehicle Model }}$}

The vehicle model used the vehicle road load equation to calculate the vehicle acceleration and speed including the terms of input force (traction force), air drag loss and grade loss. However, the rolling resistance loss was handled in the wheel model. The vehicle model was based on the Eq. 4-26: 


$$
m_{e q} \frac{d v}{d t}=F_{\text {in }}-\frac{1}{2} \rho_{a i r} C_{d} A\left(v-v_{\text {air }}\right)^{2}-\mu m g-\mathrm{mg} \cdot \sin \alpha
$$

Where: $m_{e q}$ is the vehicle equivalent mass; $\frac{d v}{d t}$ is vehicle acceleration; $F_{i n}$ is vehicle input force; $\rho_{\text {air }}$ is density of air, which is $1.3 \mathrm{~kg} / \mathrm{m}^{3} ; C_{d}$ is drag coefficient; $A$ is vehicle frontal area; $v$ is vehicle speed; $v_{\text {air }}$ is air speed; $\mathrm{m}$ is vehicle mass; $\mathrm{g}$ is acceleration of gravity, which is $9.8 \mathrm{~m} / \mathrm{s}^{2} ; \mu$ is coefficient of rolling friction; $\alpha$ is road grade.

The equivalent mass of the vehicle is the vehicle mass and the equivalent mass of the rotating powertrain components. The equivalent mass was simplified by multiplying the vehicle mass by the constant factor of 1.08 [72].

\subsubsection{Wheel Model}

Wheel model takes into account the braking force at each wheel and the friction loss of the tire, shown as Eq. 4-27.

$$
F_{\text {out }}=\frac{T_{\text {in }}-T_{\text {brake }}}{k_{\text {radius }}}
$$

Where: $F_{\text {out }}$ is wheel output force; $T_{\text {in }}$ is wheel input torque; $T_{\text {brake }}$ is brake torque; $k_{\text {radius }}$ is radius of the wheel.

\subsection{Summary}

A forward-looking quasi-static model of the 2-Mode HEV was developed and parameterized with actual data collected experimentally. The general configuration of the 2-Mode model was the same as the power split configuration. That configuration was applicable to the 2-Mode because it connects to two electric motors, the engine and the final drive. 
The difference between the 2-Mode model and power split resided in the Simulink model for the planetary gear set block. The Simulink equation set that was defined within the planetary gear set block described four clutches in this transmission. The Simulink model incorporated models for the input-split EVT mode and compoundsplit EVT mode.

Several limitations were encountered in the PSAT model of the 2-Mode HEV. The 2-Mode transmission control algorithm caused the engine to turn backward under some conditions during deceleration and idle. Although it appears that no fuel was consumed during this mode of engine operation, some modest electric power was consumed causing an error in the predicted efficiency. 


\section{CHAPTER 5}

\section{2-MODE HYBRID TRANSMISSION MODEL}

In last chapter, the models of the 2-Mode hybrid vehicle main components were introduced with the quasi-static method. In this chapter, the detailed model of the 2-Mode transmission will be introduced since the 2-Mode transmission is the most important component of the vehicle to achieve all of the hybrid functions.

\subsection{Quasi-static Model of Single Planetary Gear}

The concept of a planetary gear set in an HEV was introduced in the literature review. The planetary gear set consists of three main components: sun, ring and carrier, shown in Figure 5-1. The fundamental equation to consider the behavior of the planetary gear set is the speed relations between the sun, ring and carrier, shown in Eq. 5-1 and Eq. 5-2, which were derived from the behavior of the planetary gear set that the gears must rotate in the way to maintain a fixed ratio to the carrier body. The fixed ratio is shown in the equations, and it equals the ratio of the number of ring gear teeth and the number of sun gear teeth. The carrier speed is the weighted sum of sun gear speed and ring gear speed. The planetary gear set has two degrees of freedom because of the speed equation even though there are three rotational components.

$$
\frac{\omega_{s}-\omega_{c}}{\omega_{r}-\omega_{c}}=-\frac{N_{r}}{N_{s}}=-k
$$




$$
\omega_{s}+k \omega_{r}=(k+1) \omega_{c}
$$

Where: $\omega_{s}$ is sun gear speed; $\omega_{c}$ is carrier speed; $\omega_{r}$ is ring gear speed; $N_{r}$ is teeth number of ring gear; $N_{s}$ is teeth number of sun gear; $k$ is gear ratio of ring gear to sun gear.

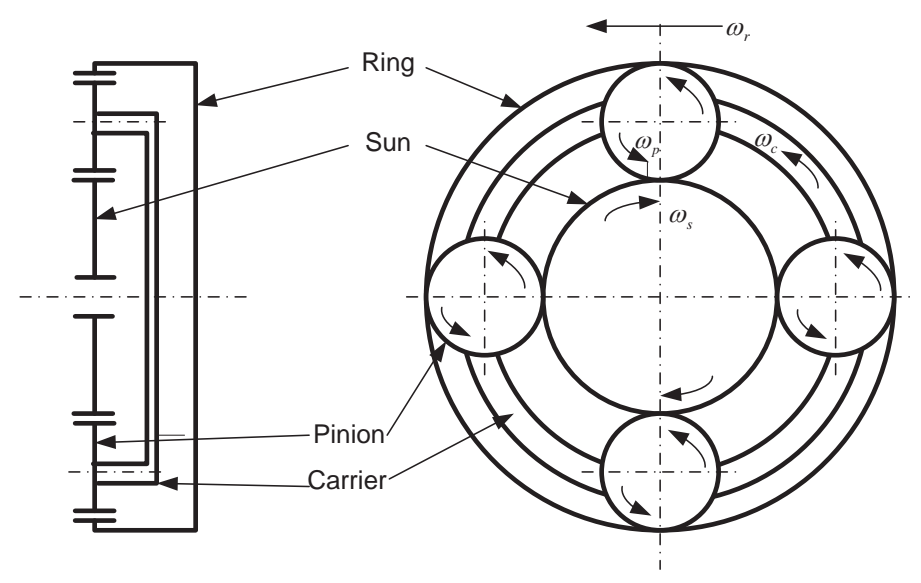

Figure 5-1: Single planetary gear set diagram.

By applying the basic law of gear meshing, the static torque relations of the sun, ring and carrier can be shown as Eq. 5-3, Eq. 5-4 and Eq. 5-5:

$$
\begin{aligned}
& \frac{T_{c}}{T_{s}}=1+k \\
& \frac{T_{c}}{T_{r}}=\frac{1+k}{k} \\
& \frac{T_{r}}{T_{s}}=-k
\end{aligned}
$$

Where: $T_{c}$ is carrier torque; $T_{s}$ is sun gear torque; $T_{r}$ is ring gear torque. 


\subsection{Model of HEV with Single Planetary Gear Set}

\subsubsection{Quasi-static Model}

The configuration of the planetary gears with the powertrain components plays an important role to achieve the desired vehicle performance. The configuration used in the Toyota Hybrid System (THS) has achieved tremendous success. The THS configuration is shown in Figure 5-2, where the engine is connected to the carrier, M/G1is connected to sun gear and M/G2 is connected to ring gear.

The engine power can be transmitted to the wheels through two different paths: the mechanical path and the electromechanical path. The mechanical path is through the ring gear, while the electromechanical path is through the sun gear. The mechanical path has higher efficiency than the electrical path for the reason that it involves fewer components and high efficiency mechanical components. The electromechanical path involves the electric motor generating and motoring processes with the power flow through the power inverter, which reduces the power transmitting efficiency.

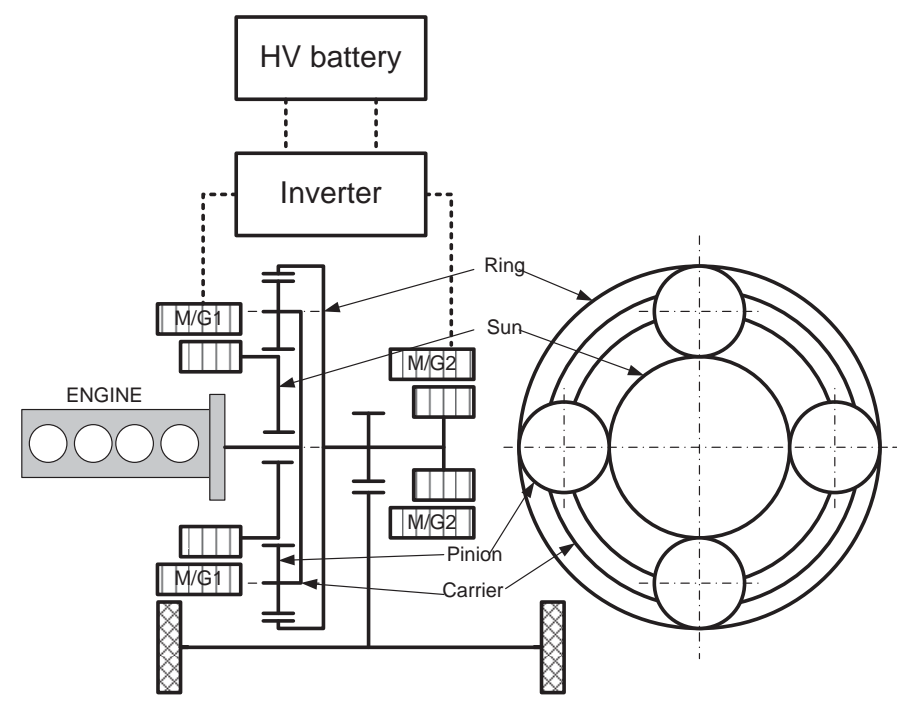

Figure 5-2: Toyota Prius vehicle layout. 
The parameters of the Toyota Prius configuration are summarized in Table 5-1, which includes the information of number of gear teeth [73], rotational speed and torque.

By applying the speed equations derived in the planetary gear set, the powertrain components speed relations are summarized in Eq. 5-6 and Eq. 5-7.

$$
\begin{gathered}
\omega_{M / G 1}+k \omega_{M / G 2}=(k+1) \omega_{e n g} \\
\omega_{M / G 2}=k_{f d} \cdot \omega_{w h}
\end{gathered}
$$

Where: $\omega_{M / G 1}$ is M/G1 speed; $\omega_{M / G 2}$ is M/G2 speed; $\omega_{\text {eng }}$ is engine speed; $k$ is the speed ratio of ring gear to sun gear; $k_{f d}$ is final drive speed ratio; $\omega_{w h}$ is wheel speed.

Table 5-1: THS powertrain configuration with planetary gear set.

\begin{tabular}{|c|c|c|c|c|}
\hline Powertrain & Engine & M/G1 & M/G2 & Wheel \\
\hline Planetary gear & Carrier & Sun gear & Ring gear & $\begin{array}{c}\text { Ring gear through } \\
\text { final drive }\end{array}$ \\
\hline Gear Teeth & $N_{c}=78$ & $N_{s}=30$ & $N_{r}=48$ & $\mathrm{n} / \mathrm{a}$ \\
\hline Speed & $\omega_{\text {eng }}$ & $\omega_{M / G 1}$ & $\omega_{M / G 2}$ & $\omega_{w h}$ \\
\hline Torque & $T_{\text {eng }}$ & $T_{M / G 1}$ & $T_{M / G 2}$ & $T_{w h}$ \\
\hline
\end{tabular}

For the control purpose, the speed of M/G1 and M/G2 can be expressed using a two by two matrix times the speed vector of the engine and wheels, shown in Eq. 5-8. In the hybrid control system, the input control signal is to adjust the engine speed, while the wheel speed is related to the vehicle speed. Hence the electric motor speed commands can be derived with the matrix equation defined below.

$$
\left[\begin{array}{l}
\omega_{M / G 1} \\
\omega_{M / G 2}
\end{array}\right]=\left[\begin{array}{cc}
k+1 & -k \cdot k_{f d} \\
0 & k_{f d}
\end{array}\right]\left[\begin{array}{c}
\omega_{e n g} \\
\omega_{w h}
\end{array}\right]
$$


With the similar method, the powertrain torque relations are summarized with Eq. 5-9, Eq. 5-10 and Eq. 5-11:

$$
\begin{gathered}
\frac{T_{e n g}}{T_{M / G 1}}=k+1 \\
\frac{T_{e n g}}{T_{r}}=\frac{k+1}{k} \\
T_{\text {eng }} \frac{k}{k+1}=T_{r}
\end{gathered}
$$

Where: $T_{\text {eng }}$ is engine torque; $T_{M / G 1}$ is $\mathrm{M} / \mathrm{G} 1$ torque; $T_{r}$ is ring gear torque.

At the ring gear, three connected components are applying torques: M/G 1, M/G2 and wheel torque $T_{w h}$ through final drive. The torque relation is shown as Eq. 5-12. The matrix format of the torque relations is shown as Eq. 5-13.

$$
\begin{gathered}
T_{w h} / k_{f d}-T_{M / G 2}=T_{\text {eng }} \frac{k}{k+1} \\
{\left[\begin{array}{c}
T_{M / G 1} \\
T_{M / G 2}
\end{array}\right]=\left[\begin{array}{cc}
1 /(k+1) & 0 \\
k /(k+1) & 1 / k_{f d}
\end{array}\right]\left[\begin{array}{c}
T_{\text {eng }} \\
T_{w h}
\end{array}\right]}
\end{gathered}
$$

Where: $T_{M / G 2}$ is M/G2 torque; $T_{w h}$ is wheel torque.

\subsection{2 $\quad$ Dynamic Model}

A single planetary gear set hybrid electric vehicle can be simplified as shown in

Figure 5-3. The planetary gear set is represented by three rotational components as Sun gear (S), Carrier gear (C) and Ring gear (G), which are respectively connected with $M / G 1$, engine and M/G2. The final drive is represented by the meshing gears with teeth number of N1 and N2. The half shaft and the wheel are represented by a rotational load as L. 


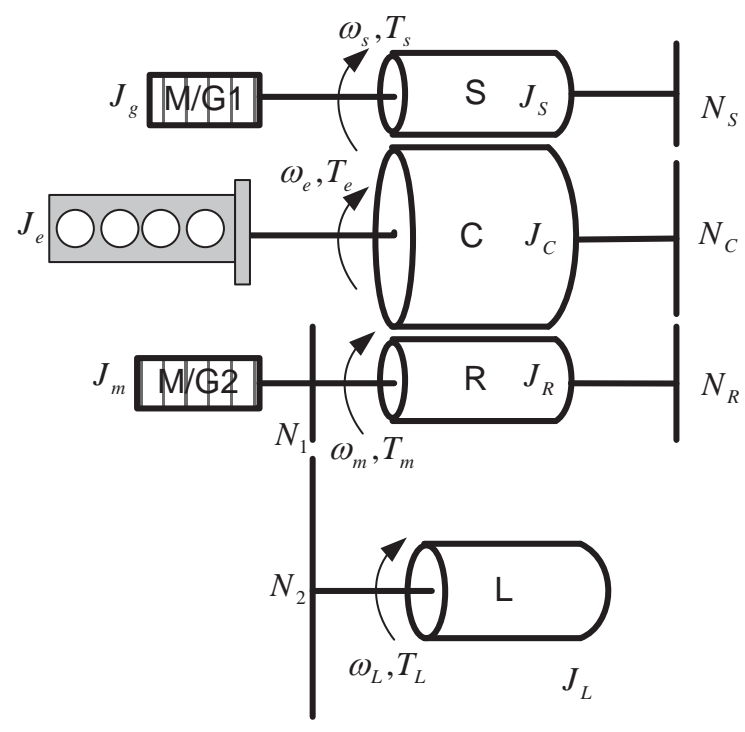

Figure 5-3: 1-mode HEV dynamic model.

The parameters of the powertrain components and planetary gears are listed in Table 5-2. The planetary gear set and the vehicle drive train was modeled as simple, stiff components without considering the elastic and damping effects.

Table 5-2: 1-mode HEV dynamic model parameters.

\begin{tabular}{|c|c|c|c|c|c|c|c|}
\hline & Sun & Carrier & Ring & M/G1 & M/G2 & Engine & Load \\
\hline Teeth & $\mathrm{N}_{\mathrm{S}}$ & $\mathrm{N}_{\mathrm{C}}$ & $\mathrm{N}_{\mathrm{R}}$ & $\mathrm{n} / \mathrm{a}$ & $\mathrm{n} / \mathrm{a}$ & $\mathrm{n} / \mathrm{a}$ & $\mathrm{n} / \mathrm{a}$ \\
\hline Speed & $\omega_{S}$ & $\omega_{C}$ & $\omega_{R}$ & $\omega_{M / G 1}$ & $\omega_{M / G 2}$ & $\omega_{\text {eng }}$ & $\omega_{L}$ \\
\hline Torque & $T_{S}$ & $T_{C}$ & $T_{R}$ & $T_{M / G 1}$ & $T_{M / G 2}$ & $T_{\text {eng }}$ & $T_{L}$ \\
\hline Inertia & $J_{S}$ & $J_{C}$ & $J_{R}$ & $J_{M / G 1}$ & $J_{M / G 2}$ & $J_{\text {eng }}$ & $J_{L}$ \\
\hline
\end{tabular}

By drawing the free body diagram (FBD) of the sun gear and the ring gear, shown in

Figure 5-4 and Figure 5-5, the dynamics equations of $\mathrm{T}_{\mathrm{M} / \mathrm{G} 1}$ and $\mathrm{T}_{\mathrm{M} / \mathrm{G} 2}$ can be expressed as Eq. 5-14 and Eq. 5-15. In the sun gear FBD, the M/G1 torque, engine torque effect on 
the sun gear and the effect of the inertia are placed on the sun gear. A positive speed direction was assumed as counter-clockwise. The engine torque effect on the sun gear acts as positive, while the M/G1 torque and the inertia effect direct to negative. The equation of motion is derived as Eq. 5-14.



Figure 5-4: Sun gear FBD.



Figure 5-5: Ring gear FBD.

$$
\begin{gathered}
T_{M / G 1}=T_{e}\left(\frac{N_{S}}{N_{C}}\right)-\left[J_{S}+J_{C}\left(\frac{N_{S}}{N_{C}}\right)^{2}\right] \cdot \dot{\omega}_{S} \\
T_{M / G 2}=T_{L}\left(\frac{N_{2}}{N_{1}}\right)-T_{e}\left(\frac{N_{C}}{N_{R}}\right)+\left[J_{R}+J_{M}+J_{C}\left(\frac{N_{R}}{N_{C}}\right)^{2}+J_{L}\left(\frac{N_{1}}{N_{2}}\right)^{2}\right] \cdot \dot{\omega}_{R}
\end{gathered}
$$

Where: $J_{S}$ is the moment of inertia of sun gear; $J_{C}$ is the moment of inertia of carrier; $\dot{\omega}_{S}$ is sun gear acceleration; $T_{L}$ is load torque; $T_{e}$ is engine torque; $J_{R}$ is the 
moment of inertia of ring gear; $J_{L}$ is the moment of inertia of the load; $\omega_{R}$ is the ring gear acceleration; $\frac{N_{1}}{N_{2}}$ is the speed ratio of $N_{1}$ to $N_{2} ; \frac{N_{S}}{N_{C}}$ is the speed ratio of sun gear to carrier.

The similar method was applied to analyze the equation of motion on ring gear, shown in Eq. 5-15. Substituting the $\omega_{R}$ with equation Eq. 5-7:

$$
T_{M / G 2}=T_{L}\left(\frac{N_{2}}{N_{1}}\right)-T_{e}\left(\frac{N_{C}}{N_{R}}\right)+\left[J_{R}+J_{M}+J_{C}\left(\frac{N_{R}}{N_{C}}\right)^{2}+J_{L}\left(\frac{N_{1}}{N_{2}}\right)^{2}\right] \cdot k_{f d} \cdot \omega_{w h}^{\cdot} \quad \text { Eq. 5-16 }
$$

Where: $J_{M}$ is the moment of inertia of motor; $\omega_{w h}^{\cdot}$ is the wheel acceleration.

The motor torque commands can be derived with the dynamics equations of motion, shown as Eq. 5-17.

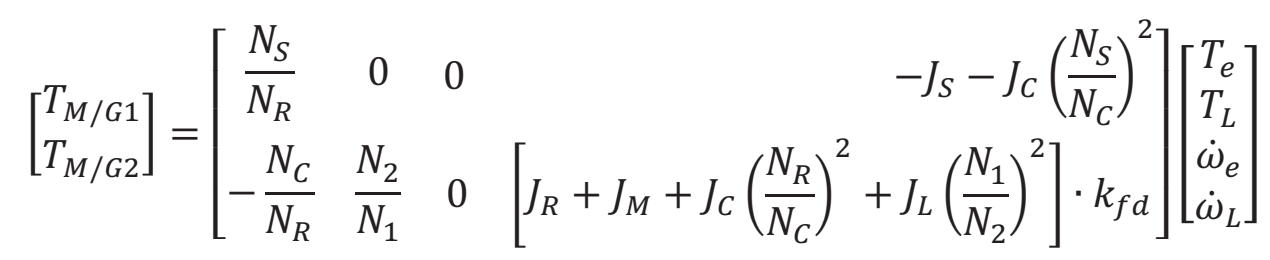

Where: $\frac{N_{C}}{N_{R}}$ is the speed ratio of carrier to ring gear; $\frac{N_{S}}{N_{R}}$ is the speed ratio of sun gear to ring gear; $\dot{\omega}_{L}$ is the rotational load acceleration.

\subsubsection{Operating Mode}

The single planetary HEV is capable of varying operating modes considering the combination of power sources and motor operation modes. The engine power can be used to drive the vehicle, charge the battery or do both. The HEV can operate with the engine power, electric power or the combination. The battery can be charged by the regenerative braking power and/or the engine power. The vehicle operation modes are discussed below:

1. Pure electric mode 
In the pure electric mode, only the battery power drives the electric motor to propel the vehicle. It can be operated in forward or reverse.

2. Engine power to charge the battery mode

Under this operation mode, all the engine power is used to charge the battery. This operation happens when the vehicle is idling and the SOC of the HV battery is low. So there is no power output to the wheels, all the engine power is used to charge battery.

3. Engine power split to drive vehicle and charge battery

Under the situation of extremely low SOC of the HV battery, the engine power is commanded higher than the need for vehicle road load power. The extra engine power is used to charge the HV battery.

4. Hybrid drive mode

In hybrid drive mode, both engine and the electric motors are powering the vehicle. This often happens when high power is requested from the vehicle, such as hard acceleration and towing.

5. Regenerative braking charging mode

Regenerative braking charging mode happens when the electric motors work as generators to charge the HV battery by capturing the braking energy.

6. Hybrid charging mode

Hybrid charging mode happens when both the regenerative braking power and the engine power charge the battery. This happens only when the HV battery SOC is extremely low that all the possible power is needed to charge the battery. 


\subsubsection{Lever Diagram}

The lever diagram was first introduced as a tool to analyze the speed and torque relations in automatic transmissions [74]. In the lever diagram, lever length is normalized as unity. The horizontal distance indicates the speed of the planetary set components. The lever diagram in Figure 5-6 (b) represents a planetary gear set in Figure 5-6 (a). The lever length is normalized as unity and the distances between the Ring to Carrier and Sun to Carrier were calculated with the planetary fixed gear ratio as $1 /(1+\mathrm{k})$ and $\mathrm{k} /(1+\mathrm{k})$, where $k=N_{R} / N_{S}, N_{R}$ is the number of ring gear teeth and $N_{S}$ is the number of sun gear teeth. It's easy to validate the speed relations in Eq. 5-6 with the lever diagram. Two triangles can be drawn as OAB and OCD, shown Figure 5-7. By applying the ratio of sides of the similar triangles, the speed relation can be derived as Eq. 5-18, which was further simplified as Eq. 5-20.

The horizontal forces shown on the inserting points indicate the torque applied on the planetary set components. By applying the force balance on the inserting points on $\mathrm{R}, \mathrm{C}$ and $\mathrm{S}$ respectively, shown in Figure 5-8. The torque equations can be easily derived the same as Eq. 5-3 to Eq. 5-5.
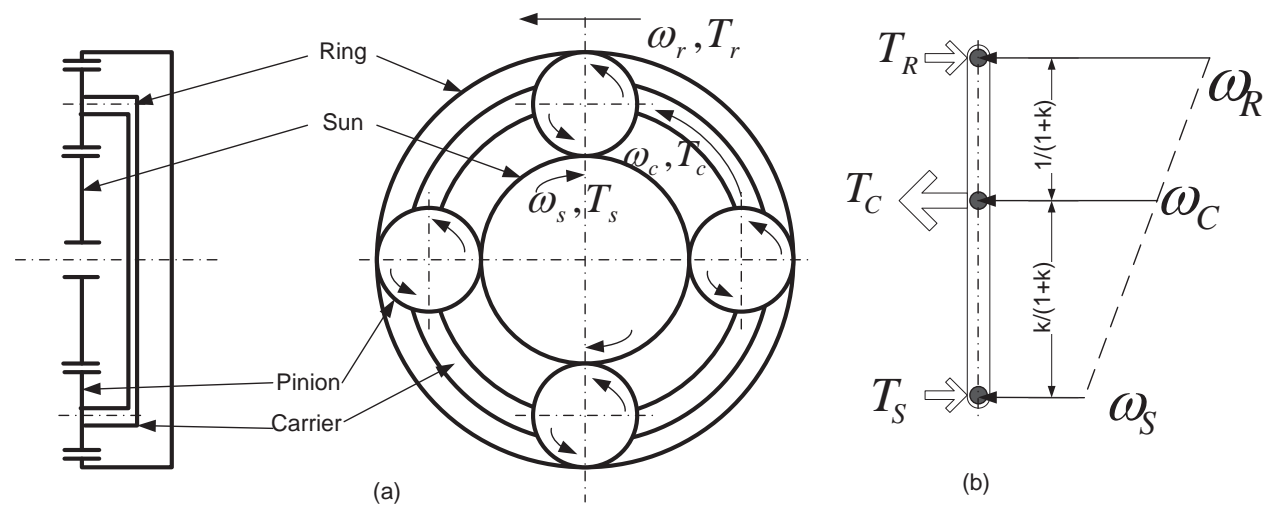

(b) 
Figure 5-6: Planetary gear set lever diagram.

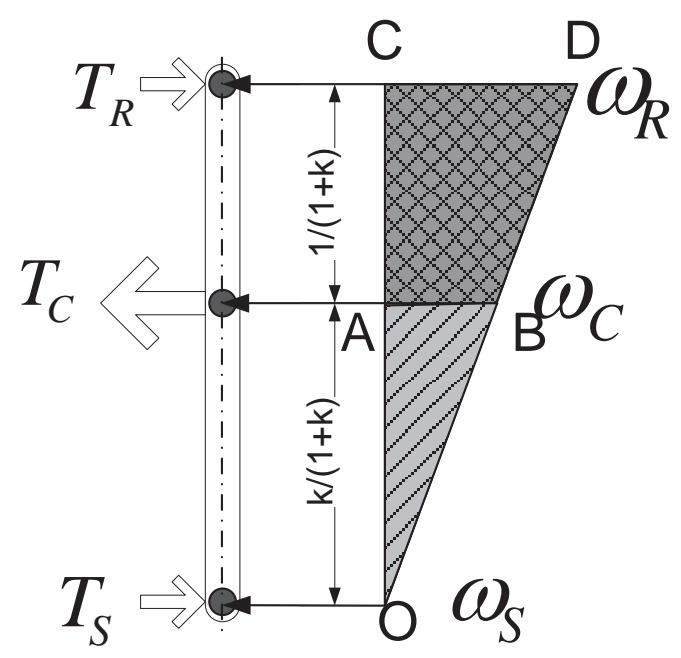

Figure 5-7: Planetary gear set lever diagram speed triangle.

$$
\begin{gathered}
\frac{\omega_{C}-\omega_{S}}{\omega_{R}-\omega_{S}}=\frac{O A}{O C} \frac{\frac{1}{1+k}}{\frac{k}{1+k}+\frac{1}{1+k}} \\
\left(\omega_{C}-\omega_{S}\right)(1+k)=\left(\omega_{R}-\omega_{S}\right) \\
\omega_{S}+k \omega_{R}=(1+k) \omega_{C}
\end{gathered}
$$

Where $k=N_{R} / N_{S}$.

The lever diagram was used to analyze the speed range of the powertrain components connected to the planetary gear set, shown in Figure 5-8. The M/G2 only rotated in the negative direction to crank the engine. The M/G2 speed was linear to the vehicle speed since the M/G2 has connected to the wheels through final drive. During the launch, the engine was off, the $\mathrm{M} / \mathrm{G} 2$ rotated in the negative direction and the $\mathrm{M} / \mathrm{G} 1$ rotated in the positive direction to start the engine. In the EV mode, the engine was off and the M/G1 rotated in the negative direction. In the low or medium speed drive, engine 
operated in a low speed range and M/G1 still rotated in the negative direction. During acceleration operation in Mode 1, the M/G1 needed to change direction from negative to positive and reach a high speed to rev up the engine. The M/G1 had much smaller inertia to quickly change the speed and direction. It's straight-forward to find out that M/G1 had a wider speed range than the engine and M/G2 for the request of EV driving and acceleration.

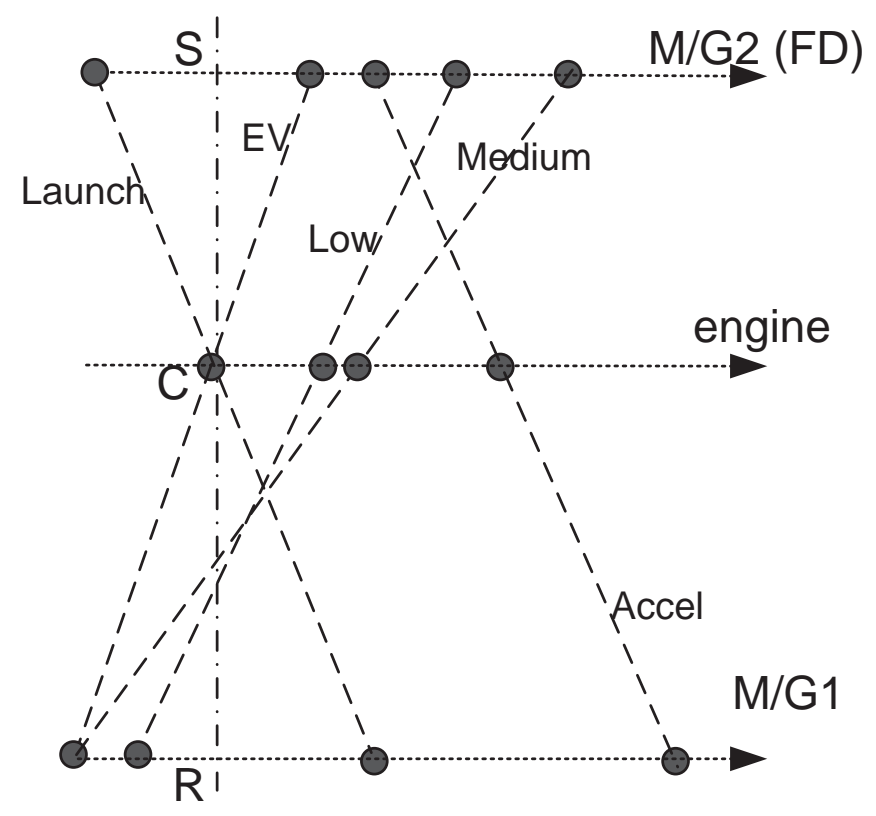

Figure 5-8: 1-mode HEV powertrain components speed limits.

\subsection{2-Mode HEV Model}

The configuration of the two planetary gears in the 2-Mode powertrain components is shown in Figure 5-9, where the engine is connected to the carrier of P1 (planetary gear 1), M/G1 is connected to the sun gear of $\mathrm{P} 1$ and $\mathrm{M} / \mathrm{G} 2$ is connected to the ring gear of P2 (planetary gear 2). Four wet-plate clutches were used to achieve extra 
fixed gears and EVT modes. However, the fixed gears were not explored in this work due to the complexity of accurate engine torque control.

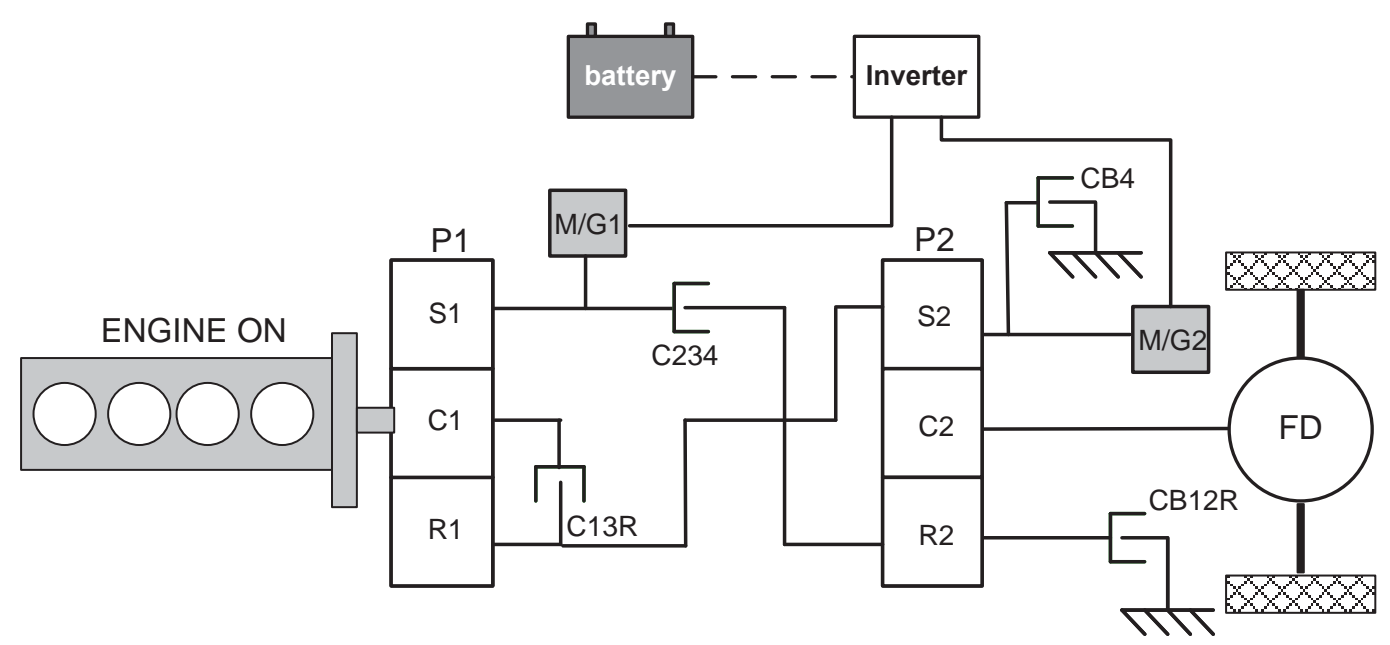

Figure 5-9: 2-Mode HEV configuration with planetary gear sets.

\subsection{1 $\quad$ Input Split (Mode 1)}

The model of 2-Mode in Mode 1 (input split mode) was simplified by connecting the engaged clutches and removing unengaged clutches, shown as Figure 5-10. Only clutch CB12R was engaged in input split mode, so the other clutches were removed from the diagram. The numbers shown in P1 and P2 gears were the teeth number of the related gears. Since there were no teeth on the carrier, the number of the carrier was calculated as the sum of sun gear teeth number and ring gear teeth number in order to keep the right speed ratios. The input split mode was similar to the Toyota Prius split configuration, except that the second planetary gear set P2 functioned to reduce the output speed further. 




Figure 5-10: Input split configuration.

Using the same method as with a single planetary gear set, the input split mode was modeled with a lever diagram as shown in Figure 5-11. In P2, the ring gear was fixed to the ground as clutch CB12R was engaged, so a fixed speed reduction was introduced in the second planetary gear set. In input split mode, engine power was split only in P1. The connecting parts between the two planetary gear sets were R1 and S2.

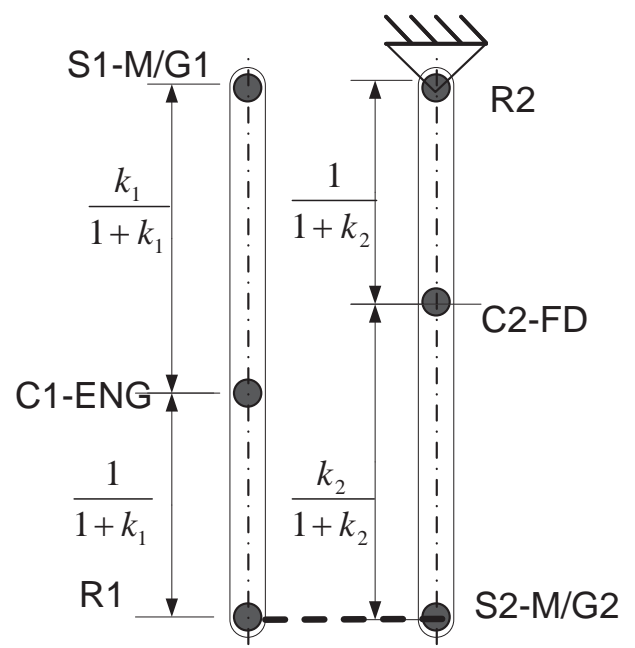

Figure 5-11: Input split mode lever diagram.

With the same lever diagram analysis method, the quasi-static model in input split mode can be expressed with Eq. 5-21, Eq. 5-22 and Eq. 5-23. Two different driving 
scenarios were shown as examples of how the lever diagram was used to analyze the speed and torque relations, shown in Figure 5-12. The figure shows the vehicle driving at low to medium speeds in input Power Split Mode with the engine off in (a) and engine on in (b). The horizontal distance from P1 inserting points S1, C1 and R1 to the left dash-dot line, represents the speeds. It worked the same way for P2. It was found that as the engine was shut down, the M/G1 speed must rotate much faster in order to keep the same vehicle speed compared with the driving with the engine on.

$$
\begin{array}{cc}
\omega_{M / G 2}=\left(1+k_{2}\right) k_{f d} \cdot \omega_{w h} & \text { Eq. 5-21 } \\
\omega_{M / G 1}+k_{1}\left(1+k_{2}\right) k_{f d} \cdot \omega_{w h}=\left(k_{1}+1\right) \omega_{e n g} & \text { Eq. 5-22 } \\
{\left[\begin{array}{c}
\omega_{M / G 1} \\
\omega_{M / G 2}
\end{array}\right]=\left[\begin{array}{cc}
k_{1}+1 & -k_{1}\left(1+k_{2}\right) k_{f d} \\
0 & \left(1+k_{2}\right) k_{f d}
\end{array}\right]\left[\begin{array}{l}
\omega_{e n g} \\
\omega_{w h}
\end{array}\right]} & \text { Eq. 5-23 }
\end{array}
$$

Where: $k_{1}=\mathrm{N}_{\mathrm{R} 1} / \mathrm{N}_{\mathrm{S} 1} ; k_{2}=\mathrm{N}_{\mathrm{R} 2} / \mathrm{N}_{\mathrm{S} 2} ; \mathrm{N}_{\mathrm{R}}$ is the teeth number of ring gear; $\mathrm{N}_{\mathrm{S}}$ is the teeth number of sun gear.



(a) Low speed (engine off)

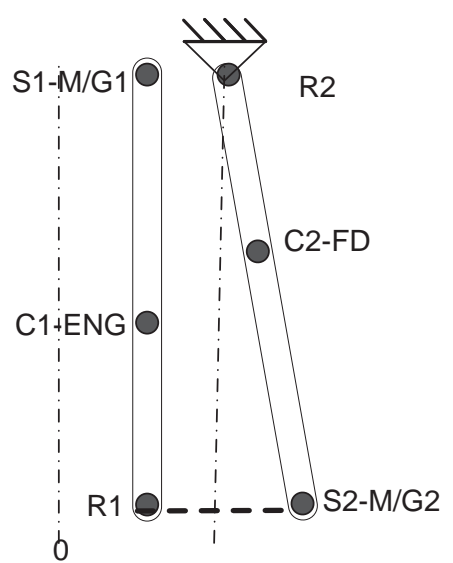

(b) Low speed (engine on)

Figure 5-12: Vehicle operation in Mode 1 with engine off and engine on. 
By applying the force balance on the inserting points on R1, C1 and S1 respectively, the torque equations were derived as Eq. 5-24.

$$
\left[\begin{array}{l}
T_{M / G 1} \\
T_{M / G 2}
\end{array}\right]=\left[\begin{array}{cc}
1 /\left(k_{1}+1\right) & 0 \\
k_{1} /\left(k_{1}+1\right) & \left(k_{2}+1\right) / k_{f d}
\end{array}\right]\left[\begin{array}{c}
T_{e n g} \\
T_{w h}
\end{array}\right]
$$

\subsection{2 $\quad$ Compound Split (Mode 2)}

The model of the 2-Mode in Mode 2 (compound split mode) was simplified as

Figure 5-13. This mode was called compound split because the engine power was split in both planetary gear sets.

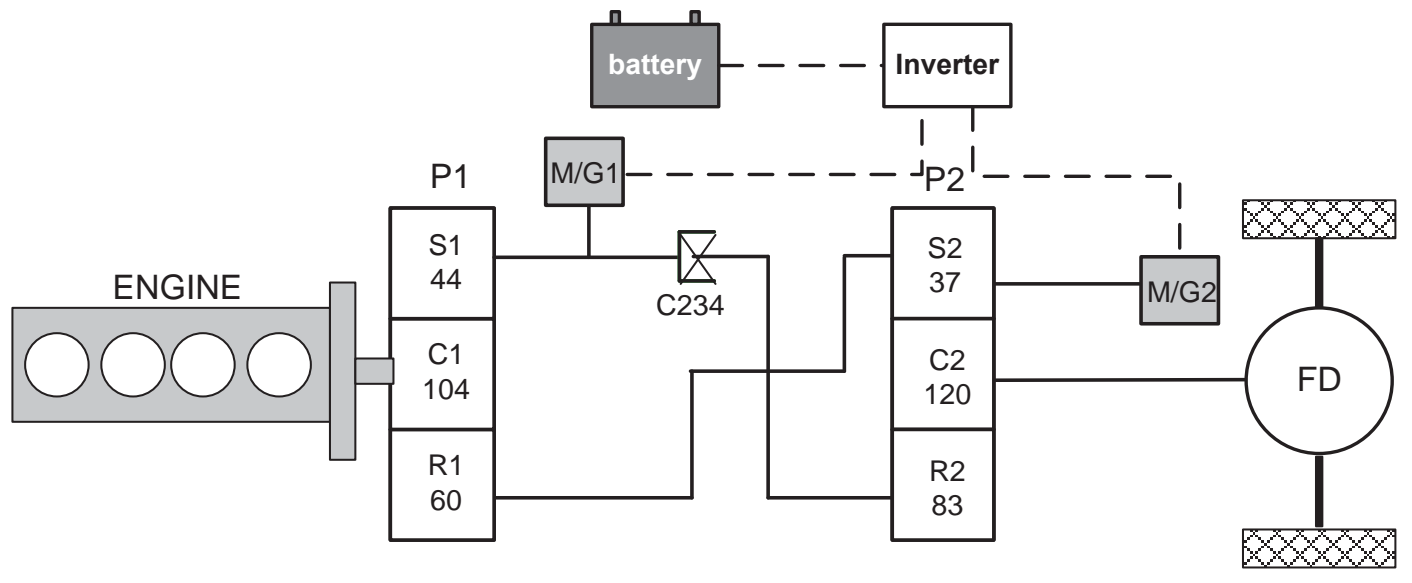

Figure 5-13: Compound split configuration.

The same method used before was applied to develop the lever diagram in the compound split mode, shown in Figure 5-14. The two planetary gear sets were connected with S1-R2 and R1-S2. 


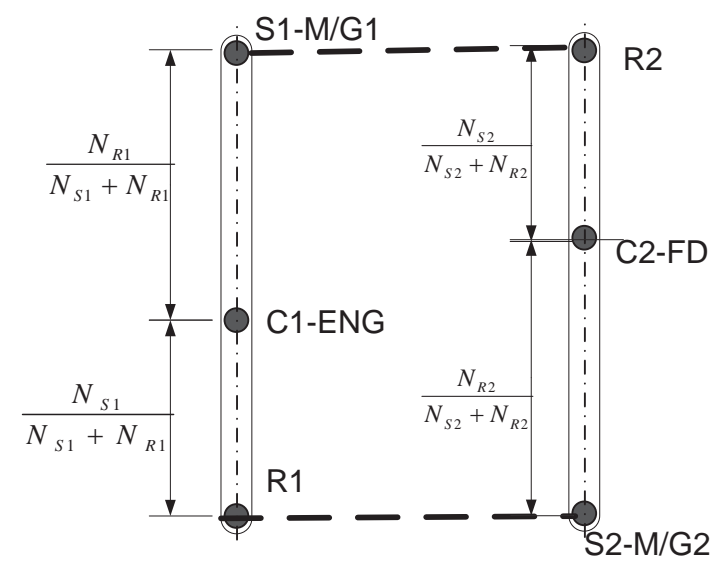

Figure 5-14: Compound split mode lever diagram.

The lever diagram was further simplified by combining the two levers, shown at the left in Figure 5-15. The combination of the two levers was made to keep both the speed and torque ratio unchanged. Applying the same speed relation analysis method used in the lever diagram, the speed and torque relations were derived as shown in Eq. 5-25 and Eq. 5-26.


Figure 5-15: Compound split mode single lever diagram. 


$$
\begin{gathered}
{\left[\begin{array}{l}
\omega_{M / G 1} \\
\omega_{M / G 2}
\end{array}\right]=\left[\begin{array}{cc}
\frac{1+k_{1}}{1-k_{1} k_{2}} & -\frac{k_{1}\left(1+k_{2}\right)}{1-k_{1} k_{2}} k_{f d} \\
\frac{k_{2}\left(1+k_{1}\right)}{k_{1} k_{2}-1} & -\frac{1+k_{2}}{k_{1} k_{2}-1} k_{f d}
\end{array}\right]\left[\begin{array}{c}
\omega_{\text {eng }} \\
\omega_{w h}
\end{array}\right]} \\
{\left[\begin{array}{l}
T_{M / G 1} \\
T_{M / G 2}
\end{array}\right]=\left[\begin{array}{cc}
-\frac{1}{1+k_{1}} & -\frac{k_{2}}{1+k_{2}} k_{f d} \\
-\frac{k_{1}}{1+k_{1}} & -\frac{1}{1+k_{2}} k_{f d}
\end{array}\right]\left[\begin{array}{c}
T_{\text {eng }} \\
T_{w h}
\end{array}\right]}
\end{gathered}
$$

Where: $k_{1}=\mathrm{N}_{\mathrm{R} 1} / \mathrm{N}_{\mathrm{S} 1} ; k_{2}=\mathrm{N}_{\mathrm{R} 2} / \mathrm{N}_{\mathrm{S} 2} ; k_{f d}$ is the speed ratio of final drive.

Typical examples of the compound split mode application during different driving scenarios are shown in Figure 5-16. All three lever diagrams indicate the same vehicle speed since the distance from C2-FD to the dash-dotted line is the same. In (a), there is no engine power flow through the electric path because the speed of M/G1 is zero. In (b), engine power is split through both electric and mechanical paths and all powertrain components are at the same speed. In (c) the engine is shut down and the vehicle is in Battery Electric Vehicle (BEV) mode. However, the speeds of M/G1 and M/G2 are high and reversed compared with (b).

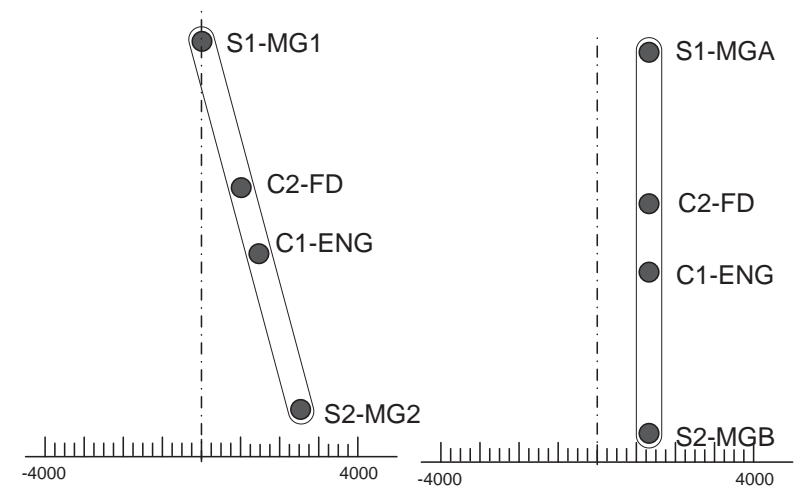

(a) mechanical path (b) split path

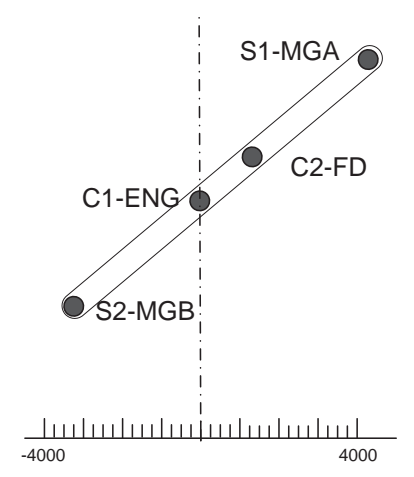

(c) engine off

Figure 5-16: Driving scenarios in compound split mode. 


\subsubsection{Powertrain Efficiency}

One of the key functions of the 2-Mode hybrid control system was to control the split of the engine power through the mechanical path and electromechanical path.

Simulation was implemented to research the power ratio transmitted by the mechanical path and the electromechanical path. The electromechanical power was split from the engine through M/G1, and it can be calculated as Eq. 5-27:

$$
P_{\text {electromechanic }}=P_{M / G 1}=T_{M / G 1} \cdot \omega_{M / G 1}
$$

Where: $P_{\text {electromechanic }}$ is electromechanical power; $P_{M / G 1}$ is M/G1 power; $T_{M / G 1}$ is M/G1 torque; $\omega_{M / G 1}$ is $\mathrm{M} / \mathrm{G} 1$ speed.

Substituting $T_{M / G 1}$ and $\omega_{M / G 1}$ in Eq. 5-23 and Eq. 5-24, the electromechanical power can be derived as Eq. 5-28:

$P_{\text {electromechanic }}=\left[T_{\text {eng }} /\left(k_{1}+1\right)\right] \cdot\left[\left(k_{1}+1\right) \omega_{\text {eng }}-k_{1}\left(1+k_{2}\right) k_{f d} \omega_{w h}\right]$

Using a similar method, the electromechanical power was summarized as Eq.

5-29 in compound split:

$$
\begin{aligned}
P_{\text {electromechanic }} & =-\left(\frac{T_{\text {eng }}}{1+k_{1}}-\frac{k_{2} k_{f d} T_{w h}}{1+k_{2}}\right)\left[\frac{\left(1+k_{1}\right) \omega_{e n g}}{1-k_{1} k_{2}}\right. \\
& \left.-\frac{k_{1} k_{f d}\left(1+k_{2}\right) \omega_{w h}}{1-k_{1} k_{2}}\right]
\end{aligned}
$$

Plotting the electromechanical power in input split (Mode 1) only, it can be seen that the electromechanical power increased rapidly in magnitude in the negative direction as vehicle speed increased, shown in Figure 5-17. The electromechanical power increased from $100 \%$ at launch and fell to zero as vehicle speed increased. As the electromechanical power dropped to zero, the transmission reached a mechanical operating point at which all of the engine power was transmitted through the mechanical 
path. At the mechanical point, the transmission speed ratio was 1.88, which was selected as the transmission shifting point between the two EVT modes. As the transmission went beyond the mechanical point, the electromechanical power increased sharply in magnitude in the negative direction.



Figure 5-17: 2-Mode powertrain efficiency in Mode 1.

Plotting the electromechanical power in combination of input split (Mode 1) and compound split (Mode 2), it can be seen that the electromechanical power didn't increase sharply after the transmission was shifted to compound split mode after the transmission went over the first mechanical point, shown in Figure 5-18. So it improved the transmission efficiency by reducing the low-efficiency electromechanical power flow from the engine to the wheel. 


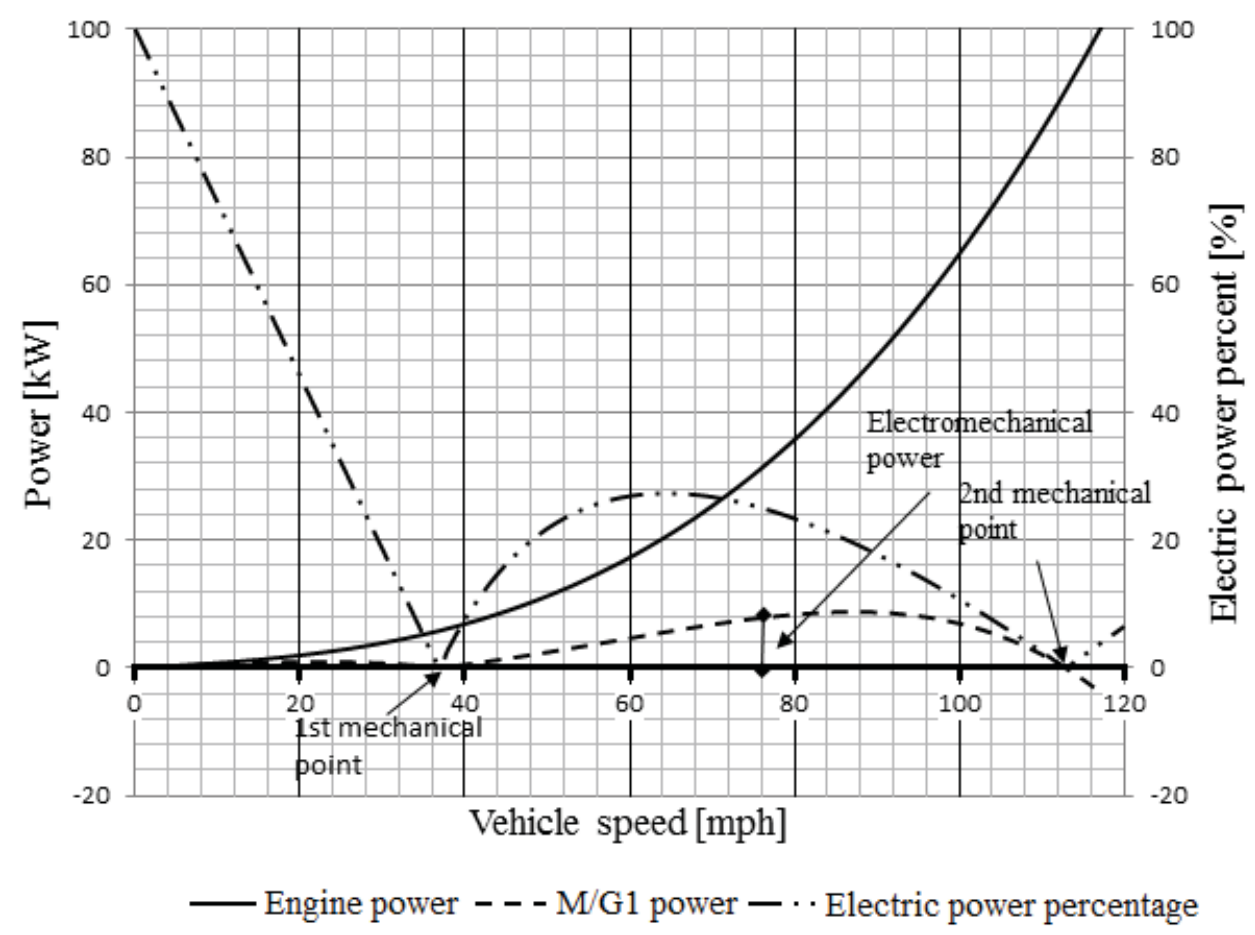

Figure 5-18: 2-Mode powertrain efficiency with 2 EVT modes.

The overall powertrain efficiency was simulated to evaluate the effects of transmission shifting and the ratio of mechanical to electromechanical power. The electric motor motoring efficiency $\eta_{m}$ and generating efficiency $\eta_{g}$ were set as constants. The mechanical path was presumed without power loss. Then the powertrain efficiency $\eta_{p t}$ was calculated as shown in Eq. 5-30. The $P_{\text {electromechanic }}$ consumed more engine power by passing through M/G1 and M/G2. The mechanical power was the total vehicle power minus electromechanical power and was assumed without losses.

$$
\eta_{p t}=\frac{P_{\text {vehicle }}}{\frac{P_{\text {electromechanic }}}{\eta_{m}} \eta_{g}+\left(P_{\text {vehicle }}-P_{\text {electromechanic }}\right)}
$$

Where: $\eta_{p t}$ is powertrain efficiency; $P_{\text {vehicle }}$ is vehicle power; $\eta_{m}$ is motor efficiency; $\eta_{g}$ is generator efficiency. $\eta_{m}$ is less than 1 for converting electric power to 
mechanical power. The $\eta_{g}$ is bigger than 1 for consuming engine power to electric power.

It can be seen that the 2-Mode powertrain had better efficiency compared with the 1-mode powertrain efficiency, shown in Figure 5-19. Using input split only, the powertrain reached maximum powertrain efficiency of $100 \%$ theoretically at the mechanical operating point, then it dropped rapidly. However, after the transmission was shifted to compound split (Mode 2), the powertrain efficiency remained high because it reduced the electromechanical power. The powertrain reached high efficiency of $100 \%$ theoretically at the second mechanical operating point.

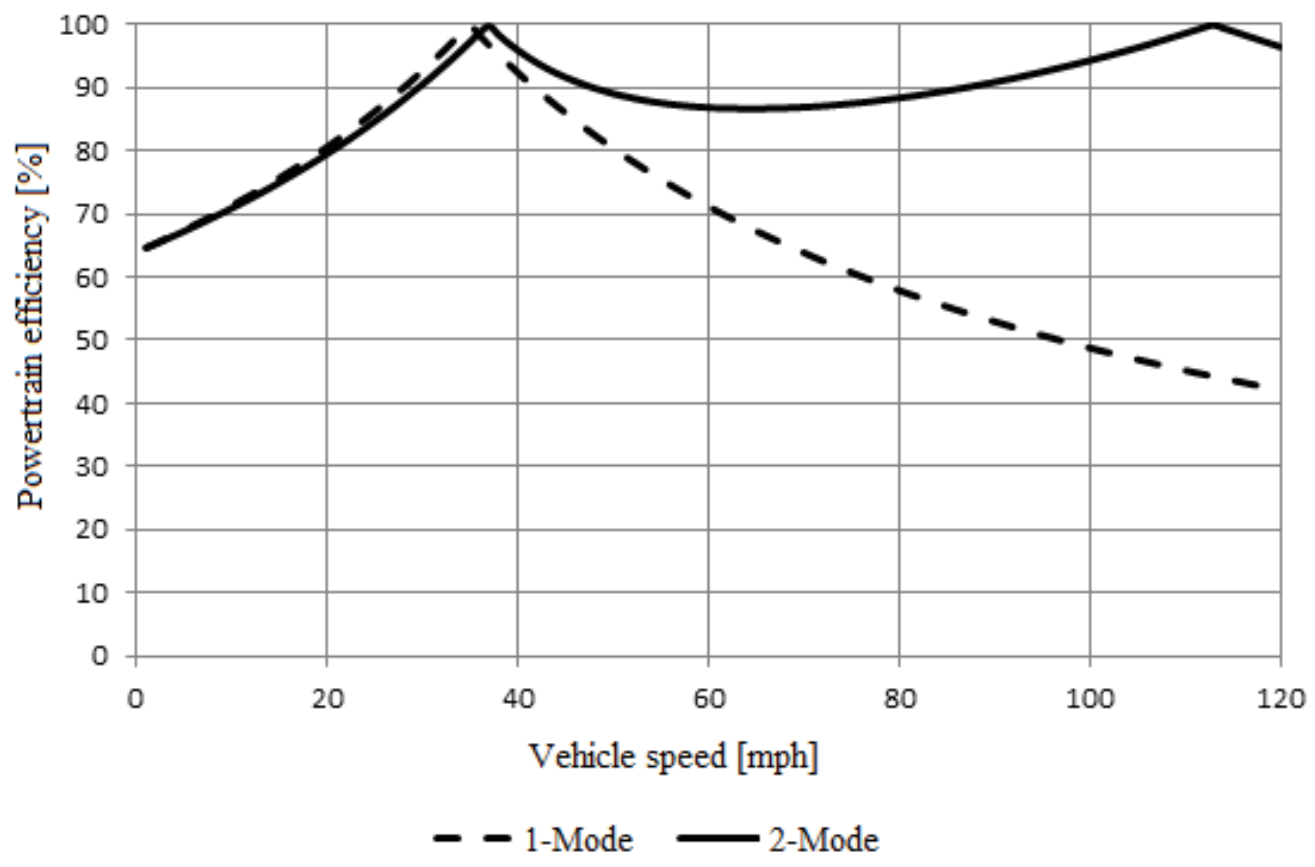

Figure 5-19: Overall powertrain efficiency using 1-Mode and 2-Mode. 


\subsection{Summary}

The planetary gear set was modeled with the details in the speed and torque relations. The lever diagram was introduced to simplify the analysis of the complicated transmission operation with planetary gear set(s).

2-Mode transmission torque and speed relations were derived from the basic equations of planetary gear set. Engine power was split into electromechanical power and mechanical power. Reducing the engine power split was the key to improve the transmission efficiency. Simulation was implemented to analyze the effect of transmission shifting and electromechanical power flow. Overall transmission efficiency was further analyzed with the assumption of constant electric motor efficiency and lossless mechanical power. The simulation results indicated the overall transmission efficiency improvement with the compound split mode implemented in the high speed range. 


\section{CHAPTER 6}

\section{2-MODE HYBRID POWERTRAIN CONTROL ALGORITHM}

\subsection{Objective of the Hybrid System Control Strategy}

Compared with the conventional vehicle, the hybrid electric vehicle control system has much more flexibility to manage the energy flow from dual power sources to the wheels. In a conventional vehicle, the engine is the only power source to propel the vehicle. However, in a hybrid electric vehicle, the vehicle can be propelled by the engine and the electric motor simultaneously or separately, and regenerative braking is exclusive to a hybrid vehicle.

The objective of the 2-Mode hybrid system control strategy was to manage the power distribution between the two power sources, engine and electric motor, to improve fuel economy while maintaining or improving the vehicle performance. In the 2-Mode hybrid electric vehicle, the vehicle had more operating modes compared with the conventional vehicle, shown in Figure 6-1. The vehicle was capable of electric vehicle (EV) mode, engine drive mode and hybrid drive mode in two EVT modes. EVT Mode 1 was operated in the low to medium vehicle speed range and in reverse. The engine stopstart was commanded in response to the vehicle power request in EVT Mode 1. EVT Mode 2 was operated in the medium to high speed, where the hybrid powertrain operated with higher efficiency. The hybrid vehicle was capable of capturing part or all of the braking energy to charge the HV battery, shown as a negative driver torque request. The regenerative braking could be operated in both EVT modes. The engine operation was separated from the unpredictable varying conditions. The 2-Mode hybrid transmission 
applied two EVT modes to allow more flexible control of the power path from the engine and electric motor to the wheels. The transmission shifting route is shown as the arrowed lines in the diagram.

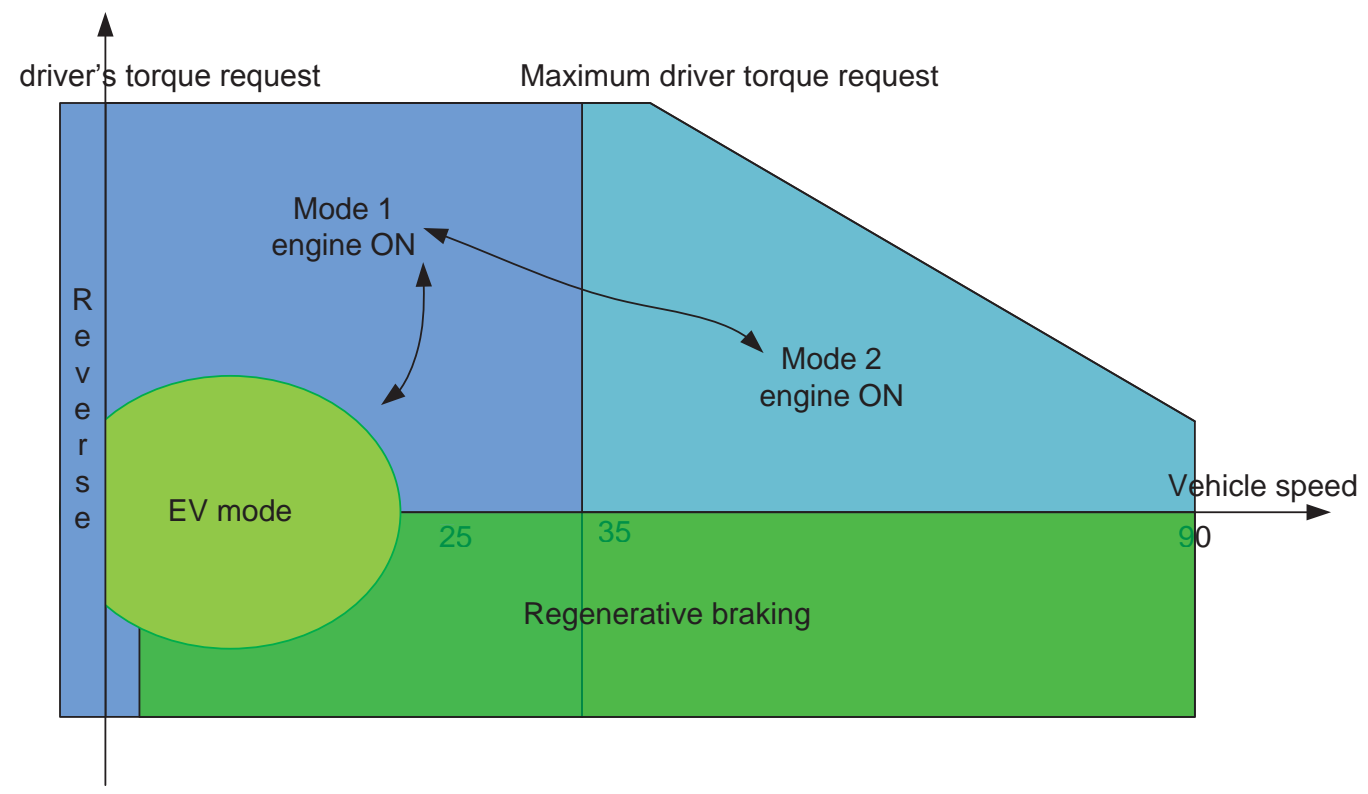

Figure 6-1: Vehicle operation modes.

\subsection{Hybrid Vehicle Modes of Operation}

\subsection{1 $\quad$ Electric Launch}

Upon the driver removing his/her foot from the brake pedal and depressing the accelerator, the vehicle is launched in electric-only mode. Under low speed driving conditions the vehicle operated in full-electric mode without starting the engine. DC power from the battery flowed to the TPIM where it was inverted into 3-phase AC power to drive M/G2. The vehicle continued to operate in electric-only mode, until additional 
power was required to accelerate the vehicle or the high voltage battery was discharged excessively. At that point the engine started to assist.

\subsection{2 $\quad$ EVT Mode 1}

After the engine was started the system operated in EVT Mode 1 which was used for low speed urban driving conditions. Utilizing an input split configuration, the engine simultaneously drove M/G1 to both generate electricity to charge the hybrid battery and provided power through the mechanical gearing in the transmission to the wheels. The energy generated by M/G1 was stored in the battery while M/G2 drew battery energy to provide additional output torque.

\subsubsection{EVT Mode 2}

As vehicle speed increased, the system shifted to EVT Mode 2. EVT Mode 2 used a compound split configuration to transfer power through the transmission during higher speed operating conditions such as highway cruising. Similar to EVT Mode 1, engine power was used to both generate electricity through the motors and provide output torque via the mechanical gearing in the transmission. A synchronous shift point through fixed gear 2 (FG2) allowed the 2-Mode transmission to shift between EVT Mode 1 and Mode 2 to improve drive quality.

\subsubsection{Regenerative Braking}

Regenerative braking was enabled in both EVT Mode 1 and Mode 2. As the driver lifted their foot from the accelerator pedal and depressed the brake pedal the electric motors were used to decelerate the vehicle by applying negative torque to the output shaft and generated electricity to charge the battery. The 3-phase AC power generated by the motor was inverted to high-voltage DC power in the TPIM and stored in 
the battery. The SCU coordinated requests for negative torque requested from the electronic brake module with electric motor and engine control functions.

\subsection{5 $\quad \underline{\text { Reverse }}$}

When the transmission shifting lever was placed in reverse, the M/G2 spun backwards and provided output torque to the wheels. When needed the engine started and M/G1 was used to charge the hybrid battery and DC power from the battery flowed to the TPIM where it was inverted into 3-phase AC power to drive M/G2.

\subsubsection{Engine Stop-start}

As the driver depressed the accelerator pedal further, demanding increased vehicle acceleration, M/G1 was used to start the engine. During the engine start event M/G1 also provided active damping to reduce torque disturbances from engine cylinder firing pulses, and M/G2 was used to dampen driveline disturbances. During this event, the HSC commanded the TPIM to draw DC power from the battery and converted it to AC power for both motors. The TPIM controlled each motor's speed and power independently. The SCU determined when to stop the engine and when to restart it based on vehicle operating conditions and optimal hybrid battery power and fuel consumption. The engine was stopped at idle and during deceleration maneuvers to improve fuel economy.

\subsection{Overall Hybrid Control System Architecture}

The overall hybrid vehicle control system architecture is shown Figure 6-2. Two control units, the SCU and ACU, were used to implement the hybrid control algorithm. The powertrain control was based on the ability to quickly respond to the driver's torque command via the accelerator pedal depression and brake pedals depression. The 
accelerator pedal position and brake pedal position signals were sent to the SCU where, based on the depression percentage and the current vehicle velocity, an output axle torque for propulsion or braking was generated. The SCU also applied the hybrid control algorithm to control engine speed/torque, engine stop-start and desired transmission gear/mode. The TPIM filtered the commands from the SCU by checking the safety violations and operation ranges of the engine, electric motors and battery. The TPIM then generated engine torque command signals, which were intercepted by the SCU and converted to engine throttle commands and sent via voltage signals to the DECM.

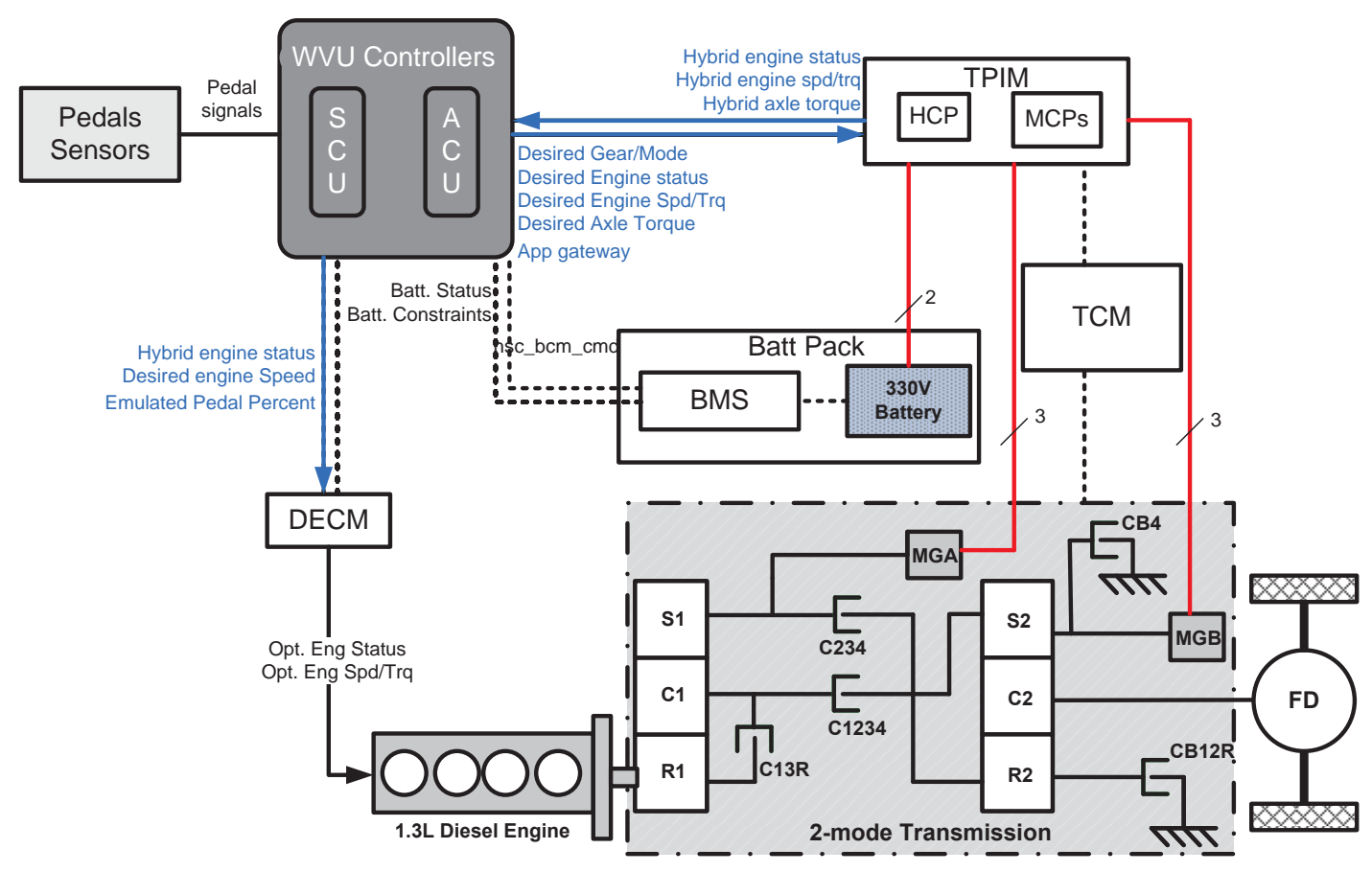

Figure 6-2: Overall hybrid control system diagram.

The SCU regulated the ratio of the power sources between the engine and the electric motor. It also determined the desired engine stop-start status and the vehicle driving mode (either propelling or braking) and relayed this information to the TPIM. 
The ACU interfaced with the battery system and implemented some other minor control functions related to the accessories.

The DECM executed the engine stop-start request, the optimal engine speed and torque command, and relayed the engine actual status to the SCU and the TPIM as a feedback loop.

\subsection{2-Mode HEV Control Software Interface}

The 2-Mode hybrid powertrain control algorithm consisted of strategic selection, tactical selection and hand-to-hand execution, shown in Figure 6-3 [75]. Strategic selection and the tactical selection were within the scope of the hybrid control algorithm, which was optimized to improve vehicle performance and fuel economy. Strategic selection included the hybrid range state determination and the transmission shifting logic. Tactical selection included the torque arbitration, engine torque optimization and engine stop-start control. Hand-to-hand execution included the M/G1 and M/G2 torque determination. For the transmission shifting control, only two EVT modes were explored in this dissertation. Fixed gear operations were not investigated due to the sophistication involved in engine torque control.

A rule-based control method was applied to simulate and implement the hybrid supervisory control algorithm, including strategic selection and tactical selection. Based on simulation and analysis of the hybrid powertrain components, it was determined that a rule-based hybrid control program would provide a feasible alternative to reach an optimal solution. The rule-based control strategy was developed based on analysis of the 2-Mode transmission dynamic model and the specifications of the engine and the energy storage system. 


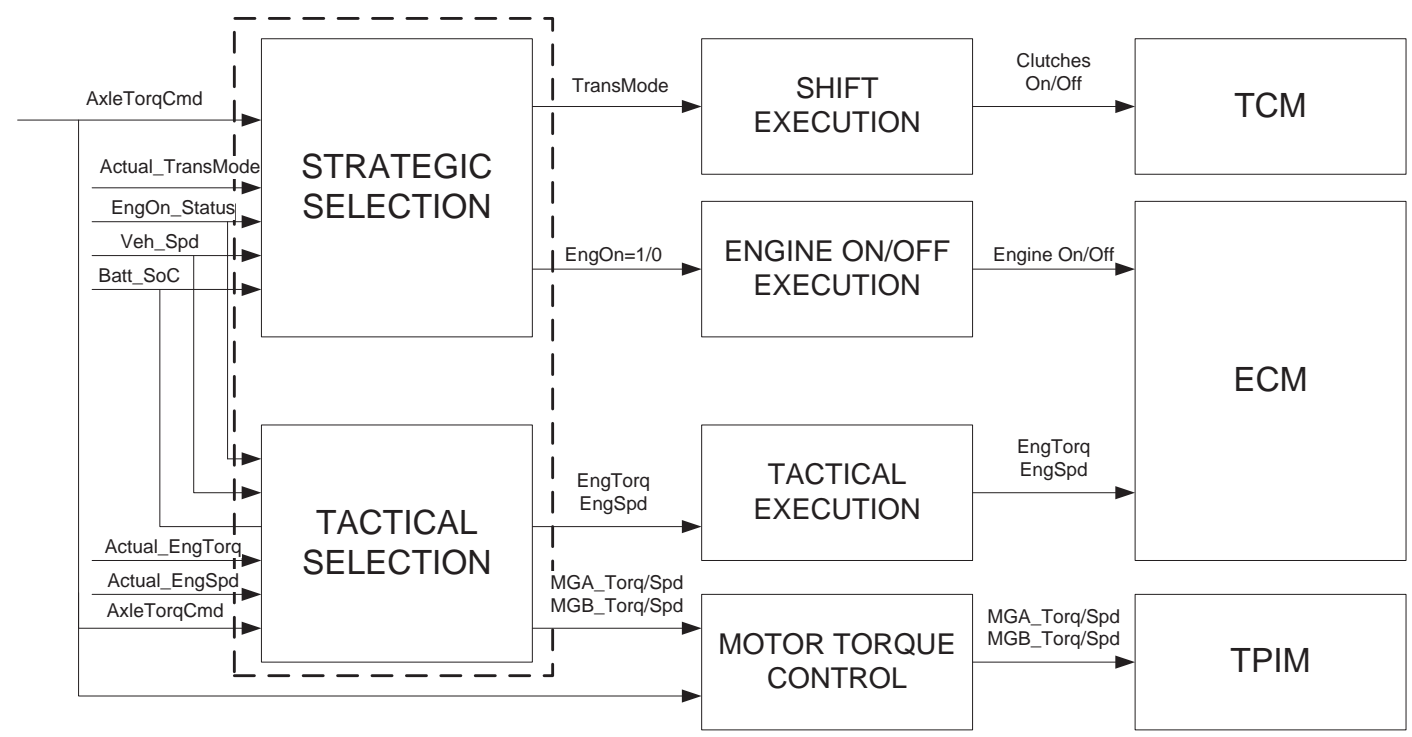

Figure 6-3: 2-Mode HEV powertrain control methodology.

There were certain constraints for the transmission shifting control algorithm.

EVT Mode 1 was used in low to medium speeds because it had higher powertrain efficiency. The engine could be either on or off in Mode 1. EVT Mode 2 had higher efficiency in medium to high speeds. In order to prevent the electric motor operating in inefficient areas, the engine must be kept on during Mode 2. In addition, when the vehicle speed was high the engine was commanded with high speeds to provide more power. When the high voltage battery SOC was low, the engine was commanded with extra power to charge the battery.

Tactical selection included controlling the optimal engine speed and torque. The engine speed command increased in order to gain more engine power as the vehicle speed increased. The engine provided as much power as possible to achieve the axle torque command and less battery power was used when the high voltage battery SOC was low.

The hand-to-hand execution was handled by the TPIM and related powertrain controllers, including the TCM. The TCM executed the shifting commands by engaging 
or disengaging the clutches in the transmission. The DECM executed engine stop-start command and responded to the optimal engine speed and torque requests from the SCU. The TPIM commanded both the torque and speed of the electric motors in the transmission based on the supervisory control algorithm and the 2-Mode transmission dynamic specifications.

\subsection{Hybrid Powertrain System Control Strategy}

The essence of hybrid control algorithm was to control the distribution of energy flow from the engine and electric motors through the mechanical and electro-mechanical paths in the 2-Mode EVT. The objectives of the control algorithm were to increase fuel economy, decrease vehicle emissions while maintaining the performance of the vehicle. Another important aspect of the control system design was to keep the powertrain components operating under the safe operation range in order to avoid powertrain components failure and possible harm to driver and passengers.

In order to improve the fuel economy, engine stop-start, transmission mode selection, torque distribution between the engine, M/G1 and M/G2 and ESS SOC sustaining were calibrated to improve the engine efficiency, and regenerative braking energy was captured as much as possible.

The overall HEV control strategy is shown in Figure 6-4. The axle torque demand was determined from a torque map based on the input of driver demand and vehicle speed, shown in Figure 6-18. The battery power availability depended on the ESS maximum current and ESS voltage. Axle power demanded was modified by the available ESS power to command engine power. The key control of this SOC sustaining 
strategy was to improve the engine efficiency since the engine provided the total power to propel the vehicle.

Desired engine status controlled the engine stop-start, which allowed the vehicle to operate under EV mode. Fuel supply was cut off when vehicle ran with EV mode.

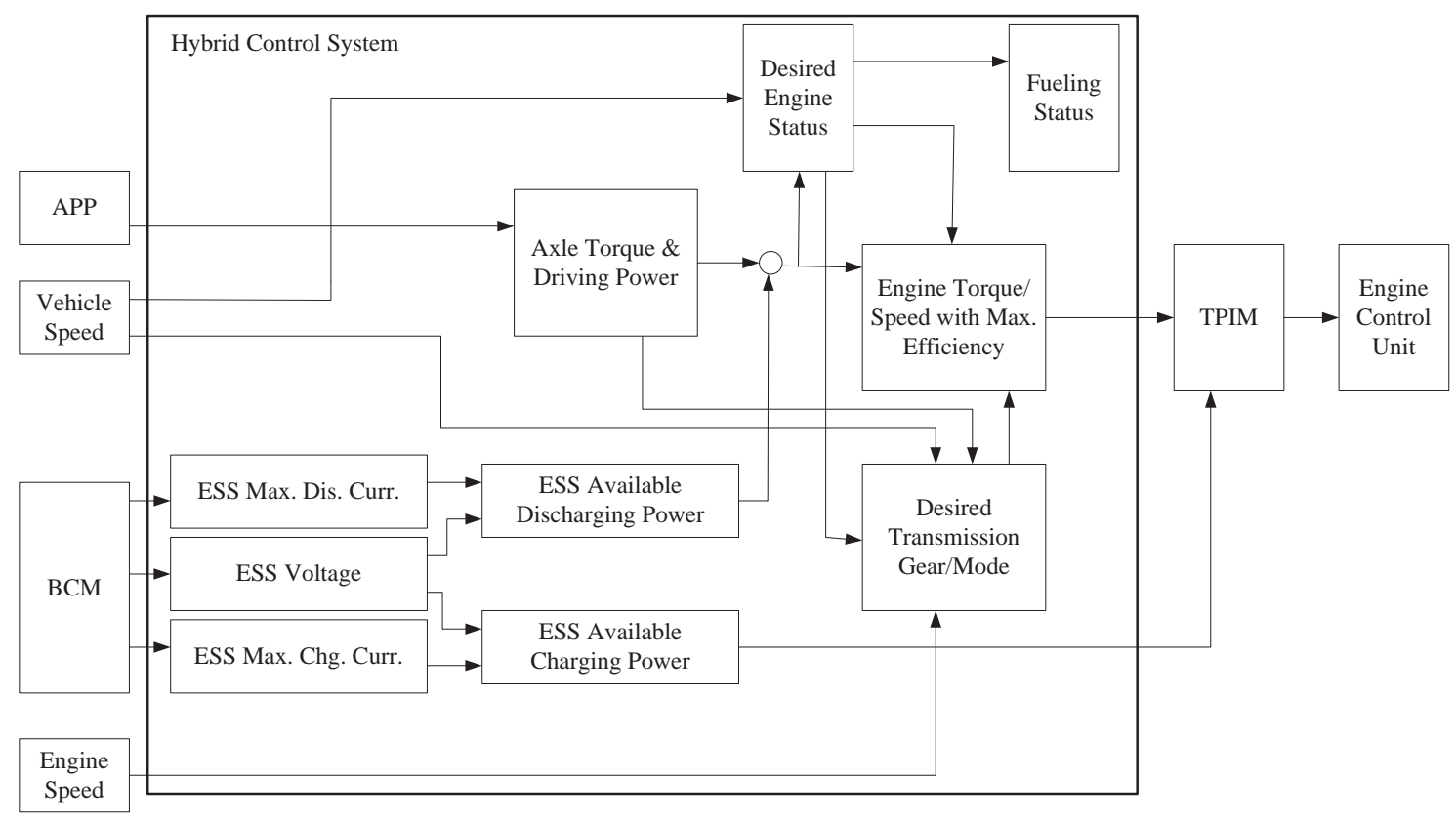

Figure 6-4: 2-Mode HEV propulsion control strategy.

\subsection{1 $\quad$ Driver Operating Mode}

The driver operating mode indicated whether the vehicle was in propelling or braking depending on the driver's inputs from the accelerator and brake pedals. The driver operating mode was determined by whether or not the driver was depressing the brake pedal. When accelerating, the brake pedal percentage was $0 \%$ and the powertrain was in the propelling mode. In the propelling mode, the SCU commanded positive axle torque to either increase or maintain the vehicle's speed. When braking, the brake pedal percentage was above $0 \%$ and the powertrain was in the Braking State. In the braking mode, the SCU commanded braking force blended with the mechanical braking and 
regenerative braking by commanding negative axle torque. When both accelerator pedal and brake pedal were depressed, the brake control logic was activated as described above. As vehicle speed was in the creep speed range within $5 \mathrm{mph}$, creep torque was applied no matter whether the vehicle was in the propelling or braking state. As vehicle speed increased above the creep speed range, creep torque was not applied.

\subsection{2 $\quad$ Propulsion Strategy}

The propulsion control strategy functioned to apply the control algorithm to distribute torque/power demands to the engine and electric motors based on current driver demand, vehicle status and ESS state of charge, in order to improve the fuel economy and keep the vehicle performance.

The driver demanded vehicle total torque/power through the acceleration/braking pedal depression. This torque/power request along with the status of the ESS and the engine speed/torque were used to determine the operation modes of the vehicle. The positive axle torque demand was for propulsion while the negative axle torque demand was for braking.

The operation mode of the vehicle and the state of the vehicle were used to determine how the torque request would be provided. The TPIM determined the torque for each electric motor and request torque from the engine. The engine torque and speed were managed by the control strategy for optimization.

\subsubsection{Engine Stop-start}

Engine stop-start control algorithm was applied in power split hybrid electric vehicles, such as in Toyota Prius Gen I and Gen II [76]. Generally, the engine is shut down when the power/torque command on engine is below a set value. The engine is 
immediately turned on when the power/ torque command is higher than a set value. In the Prius Gen 1, vehicle runs as EV at launch. The engine was turned on when engine power command was higher than $7 \mathrm{~kW}$. Then, the engine was turned off when the engine power command was less than $3 \mathrm{~kW}$. In the Pruis Gen 2, Engine stop-start function was strengthened by increasing engine turn-on-power set value to $10 \mathrm{~kW}$. The change of the engine operation points from Prius Gen 1 to Gen 2 achieved the desire to avoid low engine power levels, while this may require excess engine power to charge the battery when the vehicle propelling power wasn't demanded. The excess charge also allowed a greater percentage of electric propulsion overall and thus reduced fuel consumption.

There were certain constraints governing on the vehicle's ability to turn the engine off including battery temperature and state of charge, transmission mode, vehicle speed, and the electric motor's torque capability [77]. When the battery's state of charge dropped below $20 \%$, the engine was kept on to drive the generator to recharge the battery as well as to meet the driver's torque commands. For the FWD 2-Mode powertrain, the engine could only be turned off while the vehicle was parked, in reverse or driving in Mode 1 with the vehicle speed less than $30 \mathrm{mph}$. At vehicle speeds greater than $30 \mathrm{mph}$, the engine must be running to decrease the rotational speed of the pinions in the planetary gear set.

The electric motors can provide a fixed amount of propelling torque at a given vehicle speed. If the driver requested more torque than the electric motors can produce, the engine must be turned on to assist the motors to meet the driver's axle torque command. The engine desired status control algorithm is shown in Figure 6-5. 


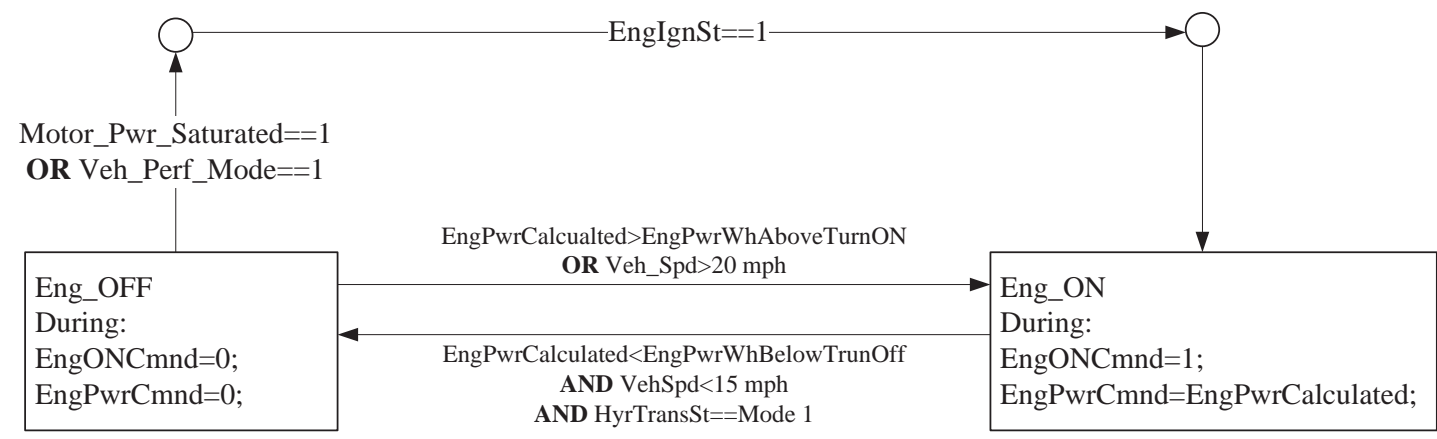

Figure 6-5: Engine desired status control algorithm.

\subsubsection{Transmission Shifting Algorithm}

The shifting process using fixed gear 2 during the shift between the two EVT modes is shown in Figure 6-6. When shifting through fixed gear 2, clutch C234 was held statically or at very low speed before the engagement. At the same time, clutch CB12R was disengaged statically or spinning at a very low speed. This process can reduce shocks of the clutches' disks during the engagement and disengagement.

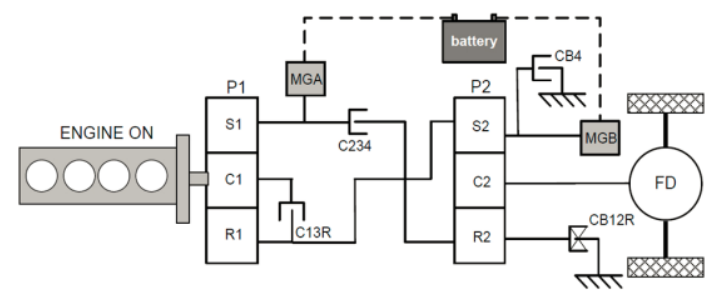

a) Mode 1

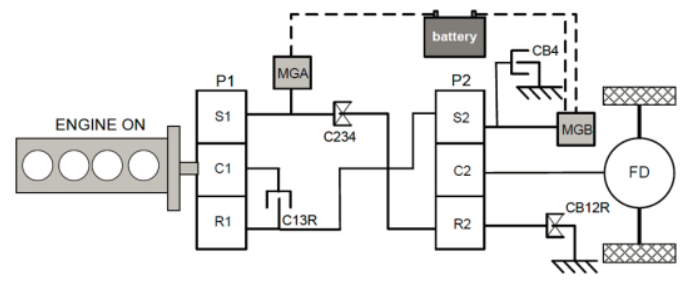

b) Fixed gear 2

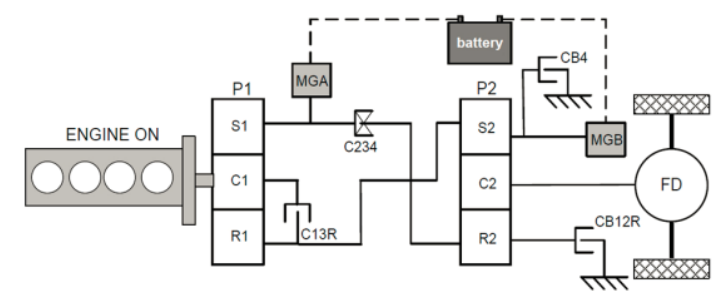

c) Mode 2

Figure 6-6: 2-Mode transmission shifting process. 
During the transmission shifting process, the engine speed was commanded to control the transmission speed ratio. At low vehicle speeds, the engine speed was commanded to produce a high transmission speed ratio to gain more torque for acceleration, shown in Figure 6-7. The transmission speed ratio in Mode 1 could be as high as infinity when the vehicle was stationary. At high vehicle speeds, the engine speed was commanded to produce a low transmission speed ratio, thus increase the engine speed to improve the engine power output. For shifting between Mode 1 and Mode 2, at a speed of $38 \mathrm{mph}$, the engine speed was commanded to the fixed gear 2 ratio of 1.88:1, shown in Figure 6-7. Slightly before and after the shifting points, the transmission speed ratio was controlled around the fixed gear 2 ratio by holding the engine speed at 2,500 rpm to guarantee a smooth shift. The transmission ratio was high at low speeds to maintain acceleration capability and low at high speeds to decrease the rotational speeds of the different planetary components inside the transmission.

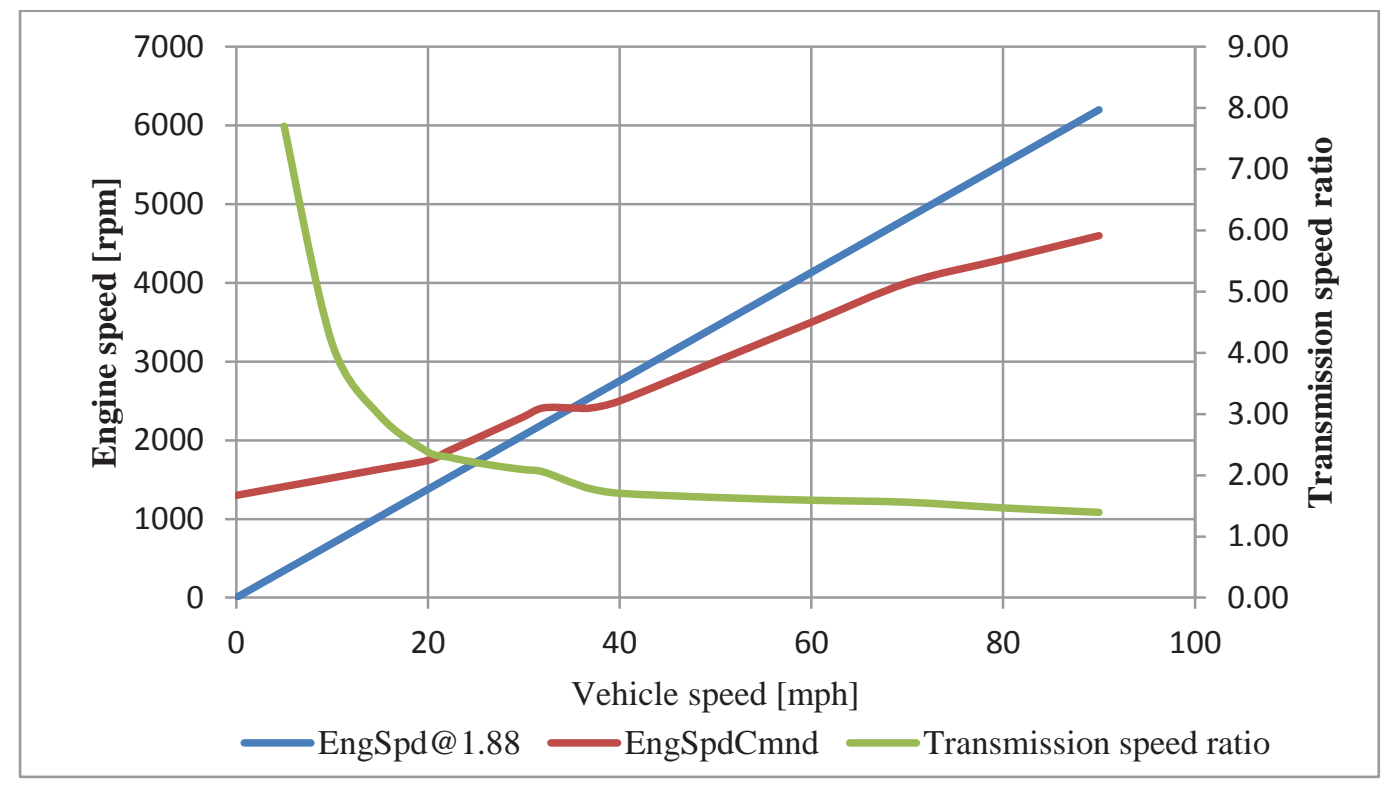

Figure 6-7: Engine speed control algorithm. 


\subsection{5 $\quad$ High Voltage Battery State of Charge Sustaining Algorithm}

A SOC sustaining control algorithm was designed to maintain the SOC around the target range, shown in Figure 9-20. The high voltage battery should not be operated within the over-charging area and over-discharging area. Between the upper limit and the target SOC was the SOC high range, where more electric power would be commanded. Between the lower limit and the target SOC was the SOC low range, where more engine power would be commanded. At very high SOC range, the vehicle would be forced to run EV mode in Mode 1. At very low SOC range, vehicle would be forced to run engine only mode.

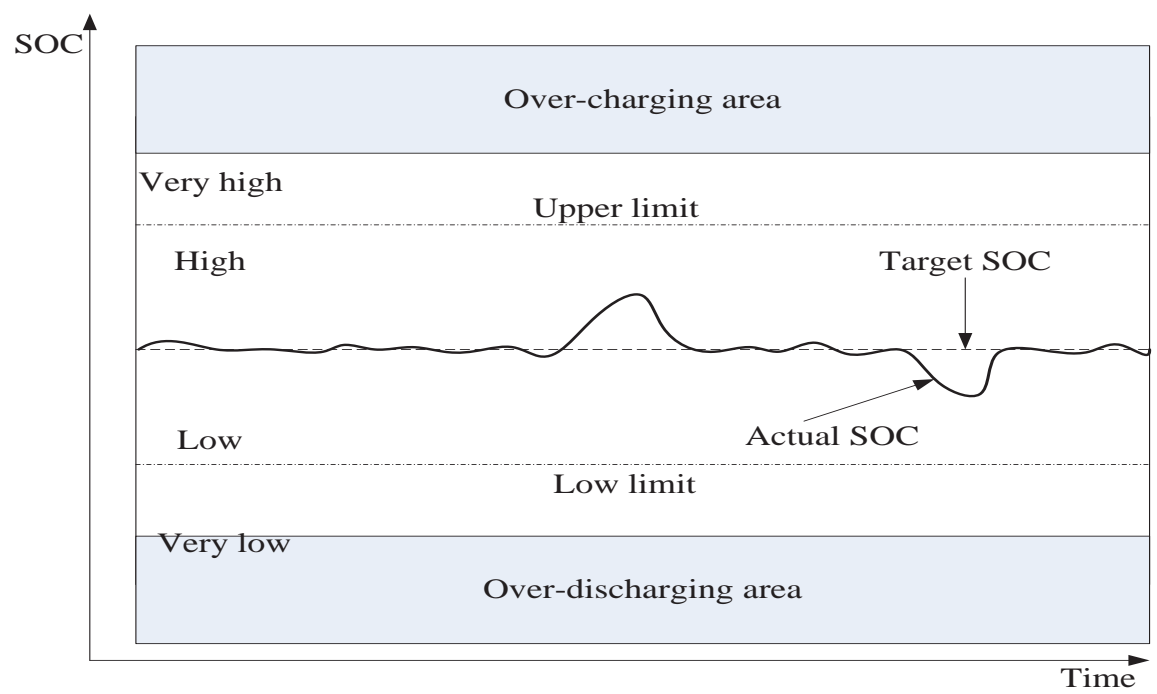

Figure 6-8: SOC sustaining control algorithm diagram.

The SOC sustaining algorithm was implemented with a PI controller to keep the high voltage battery target, shown in Figure 6-9. The ESS power command was added to the vehicle power command from the accelerator pedal to calculate the total engine power command. A positive ESS power command increased the engine torque demand 
to charge the high voltage battery when the SOC was lower than the target SOC. A negative ESS power command removed some or all load from the engine when the SOC was higher than the target SOC. The ESS SOC Control Table was a 1-D lookup table, which implemented the proportional loop of the controller. The lookup table was calibrated based on the high voltage battery specifications of charging and discharging power as a function of SOC, such that the high voltage battery would not be over-charged or over-discharged, shown in Figure 6-10. The integral loop of the controller was calibrated such that the SOC did not fluctuate abruptly around the target SOC. The integral parameter was set to 0.01 , which allowed the power command from the ESS to the engine to increase to $10 \mathrm{~kW}$ when the SOC was $10 \%$ lower than the target SOC. If the integral parameter was set too high, the power command from the ESS to the engine was too sensitive to the SOC change, thus the engine power command was modulated too frequently. If the integral parameter was set too low, the engine would not respond quickly enough to provide the power to charge the ESS, and excessive discharge of the battery could result under some driving situations. The SOC results are presented in the Vehicle On-Road Testing section.

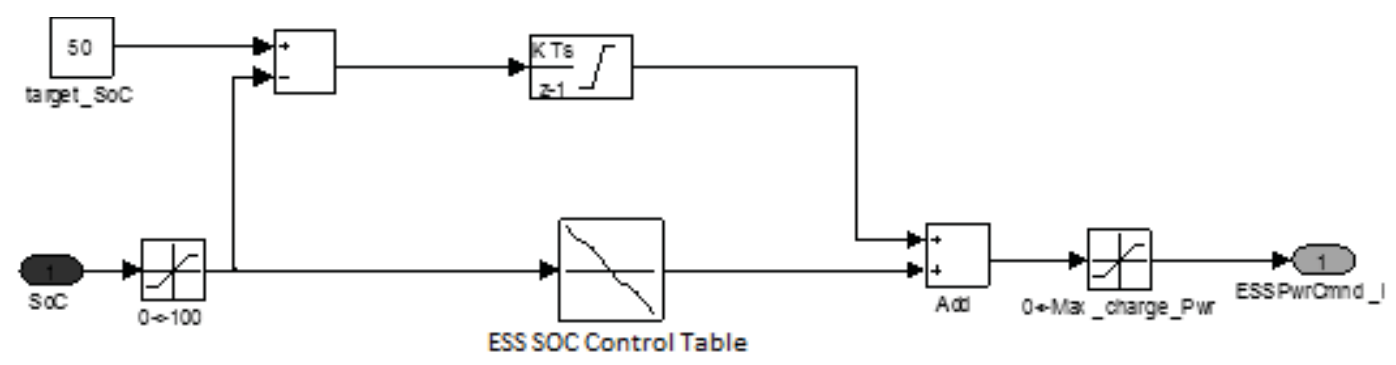

Figure 6-9: High voltage battery PI controller. 


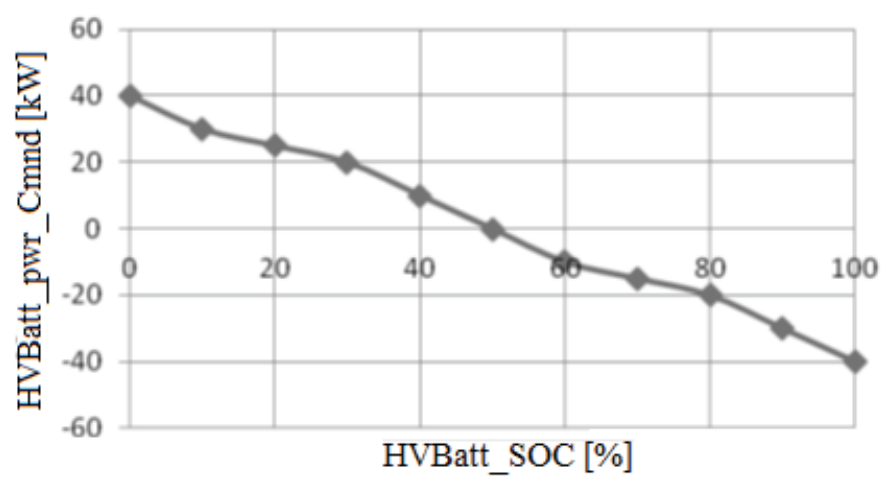

Figure 6-10: Proportional control of the HV battery SOC.

The battery control algorithm protected the battery system from overcharging or undercharging and attempted to maintain a certain target SOC. Under certain circumstances, the state of charge of the ESS may mandate charging (approaching 20\% SOC) or curtail charging (approaching 95\% SOC) in the interest of ESS protection. In order to enable best performance, a target state of charge on the order of 50\% was desired. Keeping the target SOC at 50\% would allow the discharge/charge power up to $100 \mathrm{~kW} / 70 \mathrm{~kW}$ and the discharge/charge energy up to $12 \mathrm{Ah} / 20 \mathrm{Ah}$. The allowed battery discharge/charge powers were enough to allow the maximum electric motor performance. Charging and discharging may also be limited based on the thermal state of the ESS. Charging and discharging currents were subject to a linear fold-back from full capability at $45^{\circ} \mathrm{C}$ to zero current at $65^{\circ} \mathrm{C}$. A linear fold-back on SOC was applied from $55 \%$ SOC to full charge. These fold-back limits were requested by the battery management system and executed by the propulsion algorithm. When SOC was within the normal range, the control algorithm may either deplete or charge the ESS depending on the current driving conditions. The ESS SOC was used as an input parameter in the propelling control 
algorithm to adjust the torque demand on the engine, M/G1 and M/G2, and also in the braking control algorithm to demand different levels of regenerative brake on the motors. 6.5.6 $\quad$ Regenerative Braking Strategy

In the 2-Mode hybrid electric vehicle, the traditional friction-based braking was used in conjunction with the regenerative braking to achieve the hybrid braking function. The traditional braking system was not deactivated or limited for the safety concerns. The regenerative braking effect drops off in the lower speed range, so the traditional braking is required to stop the vehicle completely. Regenerative braking capacity is limited by the electric motor status and the high voltage battery status. Regenerative braking may not happen if the battery is fully charged. Excessive braking energy may not be able to be absorbed by the regenerative braking system. Under emergency braking, the power required to be absorbed by the braking system is extremely high. The electric motor may not be capable of coping with the extra load and the battery may not be able to accept such high charging rates. Regenerative braking only catches the dynamic energy, so it is not able to hold the vehicle still on a slope. Furthermore, the friction brake is a necessary back-up in the event of failure of the regenerative brake. Under the emergency situations, the traditional friction braking system reacts much faster than the regenerative braking system to better avoid car accidents.

Unintended deceleration was considered as a hazard in the vehicle control system design phase. Unintended deceleration causes the driver to become uncomfortable. Unintended deceleration was defined as unintended deceleration changes at a rate higher than $0.2 \mathrm{~g}$ within 200 milliseconds. The extra regenerative braking force would be added 
upon the original friction braking system, which may cause unintended deceleration to affect the drivability.

In order not to add too much regenerative braking resistance to the friction braking system, the maximum deceleration added by the regenerative braking was limited below $0.6 \mathrm{~m} / \mathrm{s}^{2}$. The distribution of the deceleration was a function of braking pedal depression percentage, shown in Figure 6-11. The driver would add the same deceleration rate due to the regenerative braking effect.

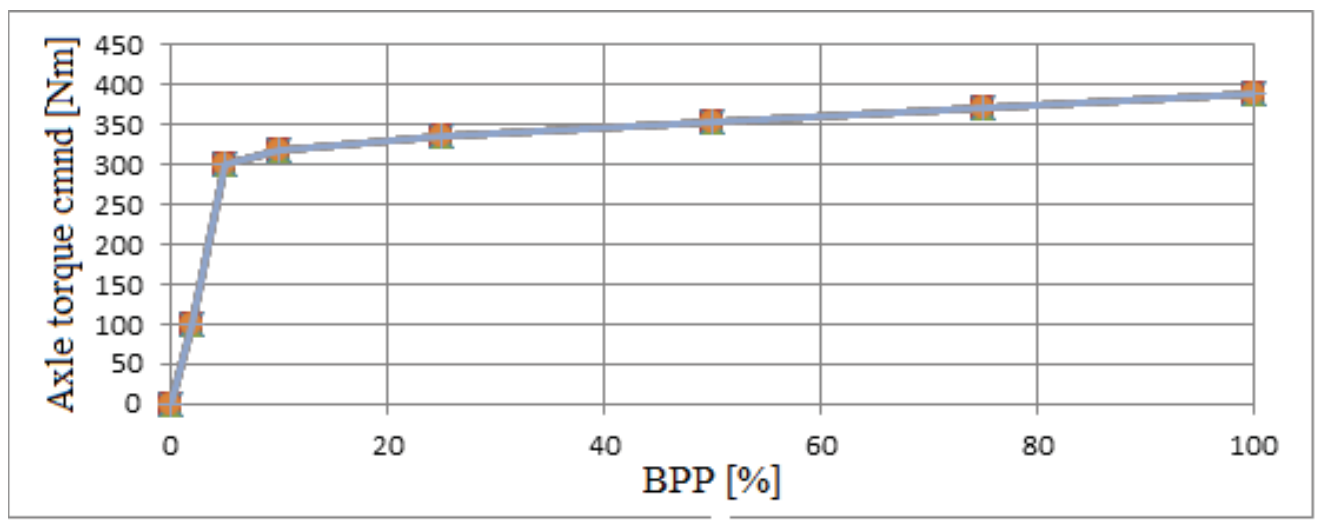

Figure 6-11: Deceleration addition by regenerative braking.

The regenerative braking powers were different at different vehicle speeds, shown in Figure 6-12. At very low speed, less regenerative deceleration was added because there was not much braking energy to be captured. At medium to high speed, more deceleration value was added to capture more regenerative braking power. No regenerative braking power was captured when the vehicle speed was less than $5 \mathrm{mph}$ since braking power was very small at low speed. With the same deceleration rate added at different speeds, more regenerative braking power could be captured at higher speed. 


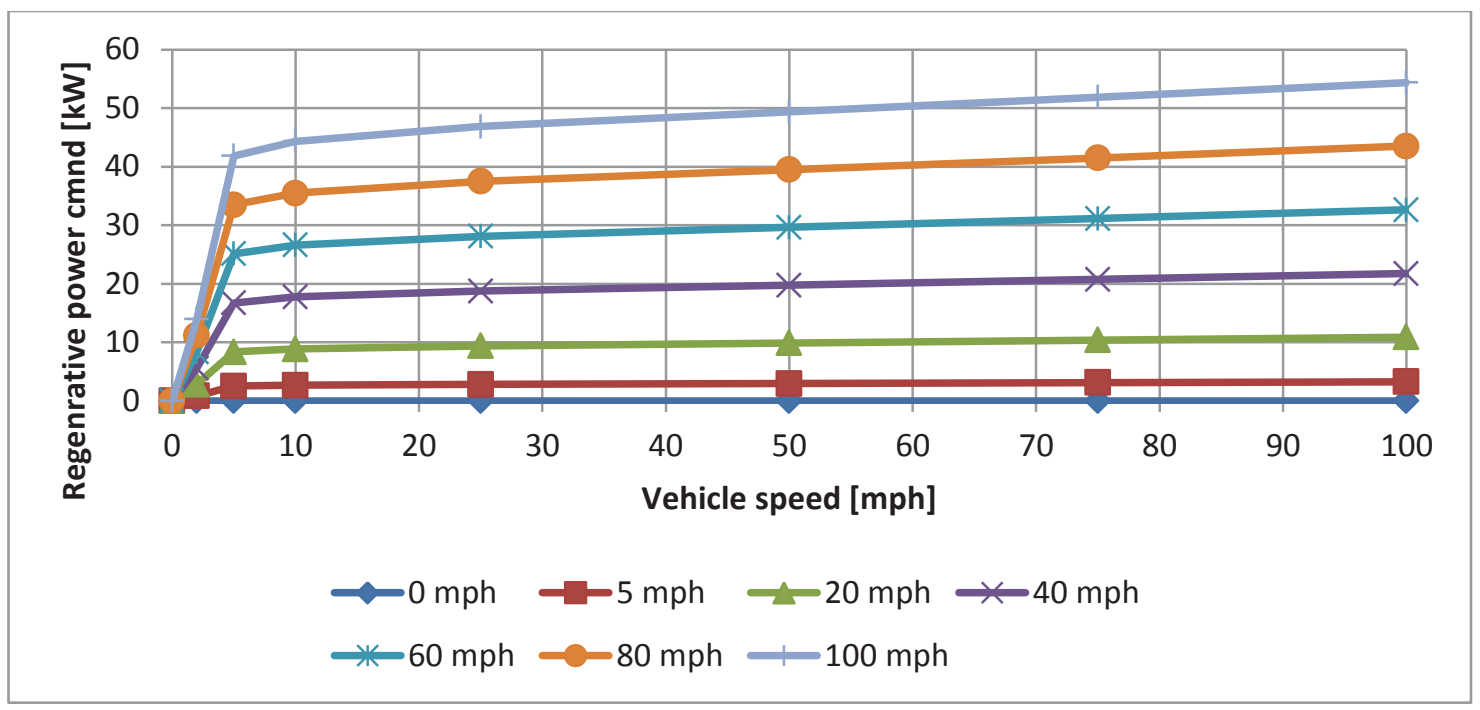

Figure 6-12: Regenerative braking power capture.

The maximum regenerative power was limited by the electric motor capability and the high voltage battery maximum charging current and charging power. The battery maximum charging power was limited by the maximum charging current since the battery voltage was relatively constant across the SOC operation range. The maximum charging current was a function of battery SOC and temperature. The high voltage battery had constant maximum charging current and short term maximum charging current (such as 10 s), shown in Figure 6-13 and Figure 6-14.

A heat index factor was introduced to interpret the battery maximum charging current between the constant maximum charging current and the short term maximum charging current. The heat index factor, $\tau$, is shown in Eq. 6-1, where $\mathrm{k}$ is the time constant that adjusts the short term maximum charging current to be saturated in predetermined time, such as $10 \mathrm{~s}$. The instantaneous maximum charging current was determined as Eq. 6-2. 


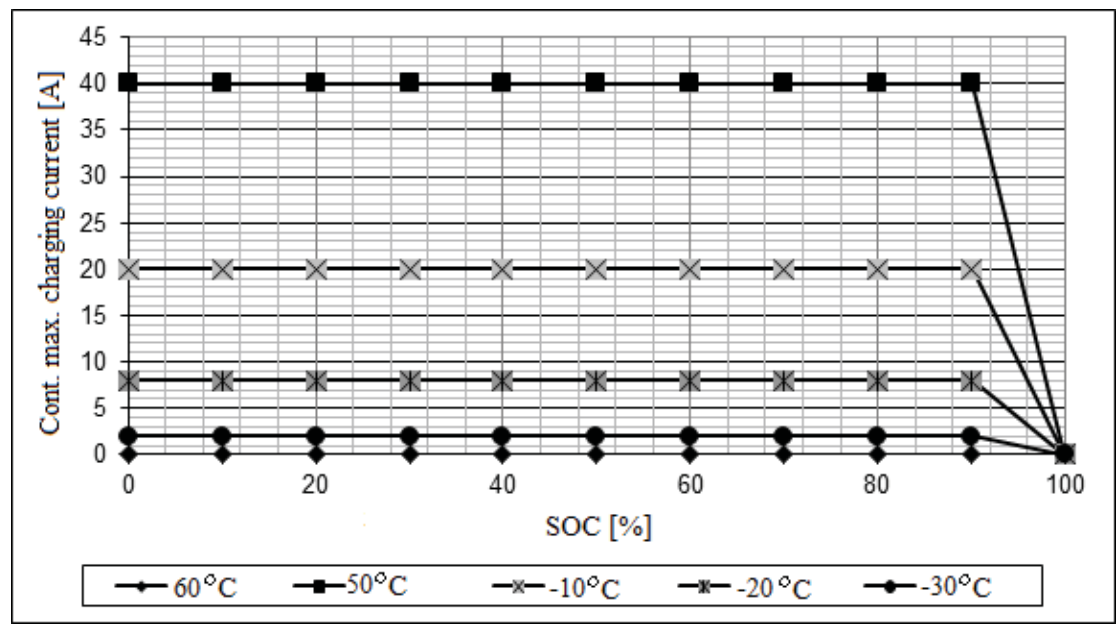

Figure 6-13: Constant maximum charging current.

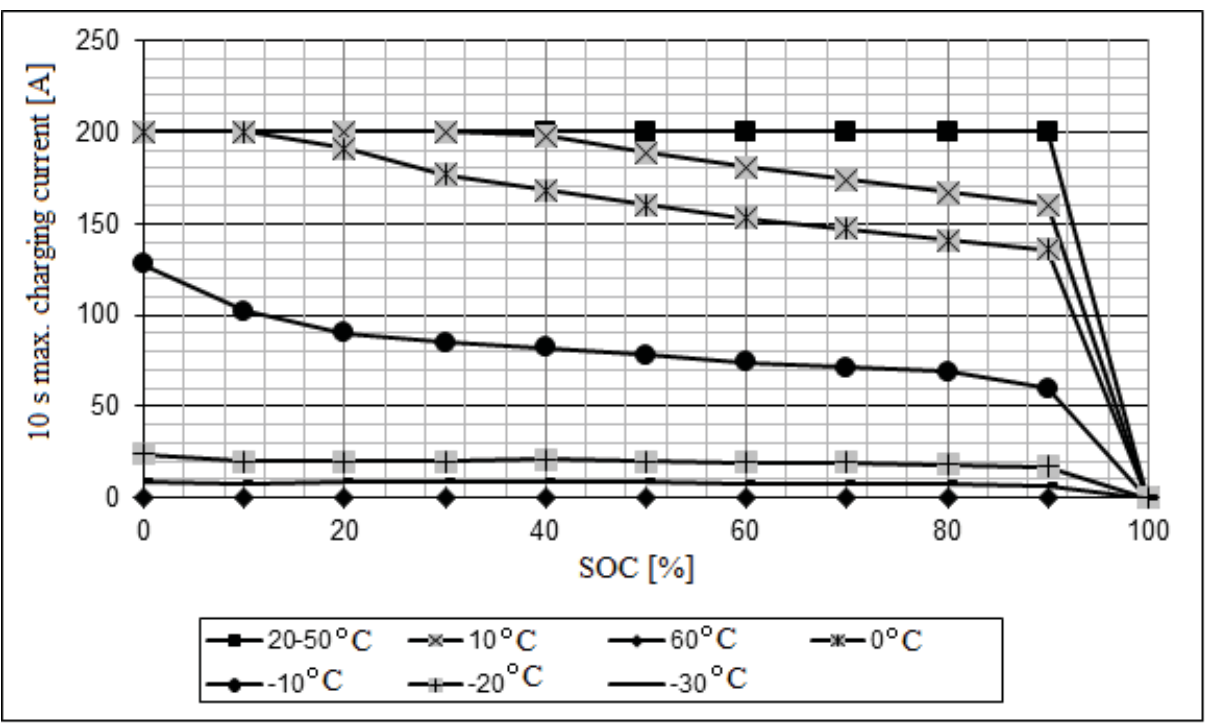

Figure 6-14: Short-term maximum charging current.

$$
\tau=\int k\left(\frac{\text { curr } \_r e q}{\text { cont_curr }}-1\right)
$$

max_chg_curr

$$
=\text { cont_curr } \cdot \tau+\text { short_term_curr } \cdot(1-\tau)
$$


Where: $\tau$ is heat index unit; $k$ is time constant; $c u r r \_r e q$ is current request; cont_curr is maximum constant current; max_chg_curr is instantaneous maximum charging current; short_term_curr is short term maximum charging current.

The electric motor charging power was limited by the maximum generating torque and the current speed. The electric motor maximum generating torque is a function of motor speed. The electric motor maximum generating torque had constant maximum generating torque and short term maximum generating torque (for example $10 \mathrm{~s}$ ), shown in Figure 6-15. A similar heat index factor was introduced to interpret the electric motor maximum generating torque between the constant and short term values. And the time constant $\mathrm{k}$ was calibrated to saturate the short term time to be the predetermined time, 10 s. The instantaneous maximum generating torque was calculated as Eq. 6-3.

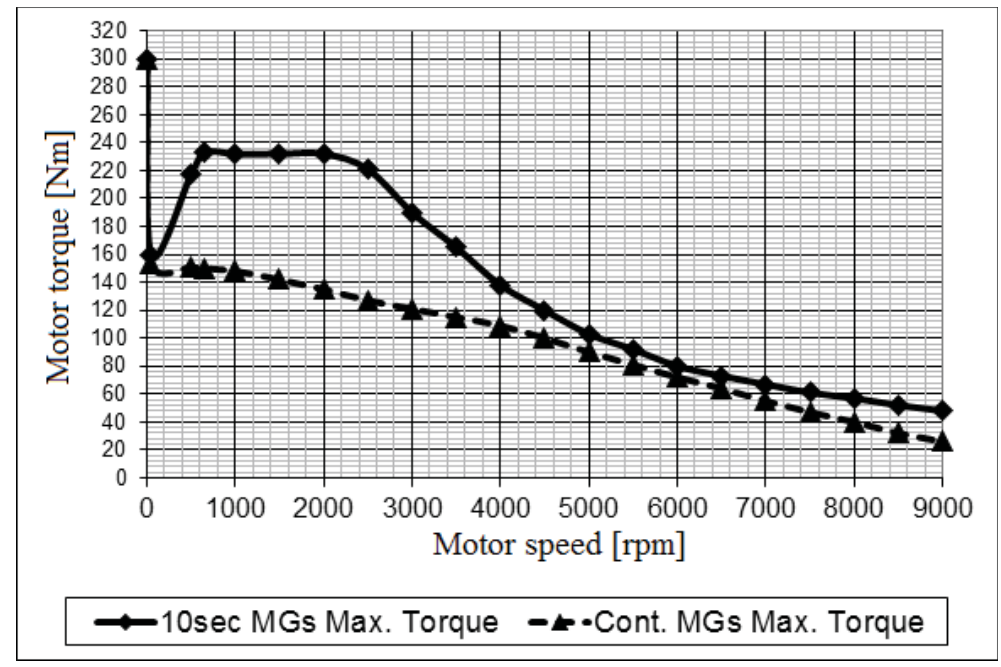

Figure 6-15: Electric motor constant maximum generating torque and short term maximum generating torque.

$$
\max _{-} \text {gen_torq }=\text { cont_torq } \cdot \tau+\text { short_term_torq } \cdot(1-\tau) \quad \text { Eq. 6-3 }
$$

Where: $\max _{\text {gen_torq }}$ is instantaneous maximum generating torque; $\tau$ is heat index factor. 
The Brake control strategy was visualized in Figure 6-16. After the brake was activated by reading the brake pedal percentage (BPP), the hybrid system controller monitored if SOC was lower than the maximum battery's SOC. When the SOC was lower, it would enable the regenerative brakes. Then the control system would check if the commanded regenerative braking charging power was less than the high voltage battery available charging power and electric motor charging power limit. The regenerative braking power command would be the minimum of the original braking power request, available battery charging power and electric motor charging power limit.

\begin{tabular}{|c|c|c|c|}
\hline \multicolumn{4}{|c|}{$\begin{array}{l}\text { Input of APP, BPP, DischargeBuffer, BattTemp, BattSOC, BattVolt and } \\
\text { MaxDischCurrent }\end{array}$} \\
\hline \multicolumn{4}{|l|}{ Yes } \\
\hline \multicolumn{4}{|l|}{ Yes } \\
\hline $\begin{array}{lll}\text { Yes } & \text { P_cl } \\
\end{array}$ & chg_Bat_lmt & $\begin{array}{c}\text { P_chg_cmd } \\
=0 ;\end{array}$ & $\begin{array}{c}\text { P_chg_cmd } \\
=0 ;\end{array}$ \\
\hline P_chg_cmd; & P_chg_cmd=P_chg_Bat_lmt & & \\
\hline Yes P_ch & _chg_EM_lmt & & \\
\hline P_chg_cmd; & P_chg_cmd=P_chg_EM_lmt & & \\
\hline
\end{tabular}

Figure 6-16: Regenerative braking control algorithm.

\subsection{7 $\quad$ Axle Torque Command}

The axle torque command was the driver's torque request to drive or stop the vehicle. The axle torque command can be either positive or negative. A positive axle torque command represented adding torque on the vehicle to gain acceleration. A negative axle torque command represented subtracting torque on the vehicle to gain deceleration, or move the vehicle backwards. 
The axle torque request was obtained from both Accelerator Pedal Position (APP) and current vehicle speed. The maximum axle torque command at different vehicle speed and 100\% APP was defined based on vehicle different operating modes, shown in Figure 6-17. The EV mode was limited in 0-20 kph and the vehicle performance was improved to the line EV 10-second Max. The vehicle acceleration capability was improved because the EM could provide maximum torque at $0 \mathrm{rpm}$, while the IC engine provided limited engine torque at idle speed.



Figure 6-17: Maximum axle torque command.

Linear interpolation was applied to determine the axle torque command at different APP percentage between 0\% and 100\%, shown in Figure 6-18. When APP percentage was at $0 \%$, the axle torque command was not zero. An initial torque value, creep torque, was set to provide vehicle creep and hill holding. The torque value was calibrated later to provide reasonable creep speed and enough torque to hold the vehicle on a hill. 


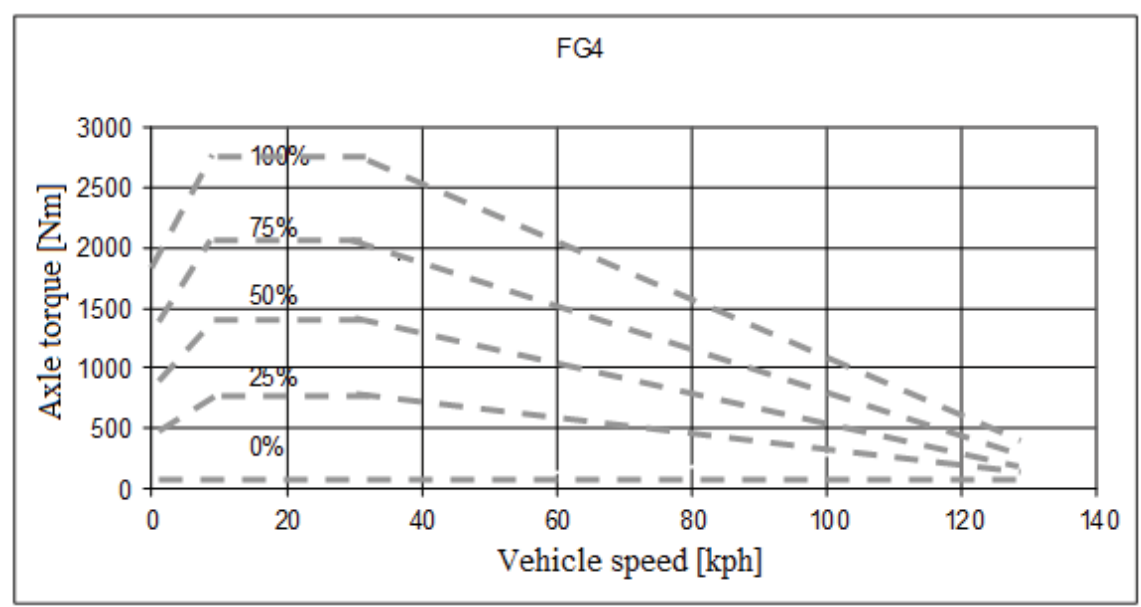

Figure 6-18: Axle torque command with different APP.

\subsection{Summary}

This chapter summarized the hybrid control system developed to drive the vehicle. The objective of the hybrid control system was to improve the fuel economy and maintain the performance. Drivability was considered to improve the vehicle drive quality.

The vehicle was capable of different operation modes to achieve hybrid functions. The hybrid vehicle achieved operation in electric launch, EVT Mode 1, EVT, Mode 2, regenerative braking, reverse and engine stop-start. The optimal operation of different modes helped vehicle to achieve the control system design objectives. 


\section{CHAPTER 7}

\section{SIMULATION AND DEVELOPMENT OF AUXILIARY CONTROL UNIT AND BATTERY MANAGEMENT SYSTEM}

\subsection{Introduction}

The 2-Mode hybrid electric vehicle introduced a 330 V DC high voltage system in the conventional vehicle, including a high voltage battery, an inverter and two electric motors. In the 2-Mode hybrid control system, one of the main functions was to integrate the high voltage battery control system with the conventional vehicular control system. The controller used to integrate the high voltage system was the ACU.

This chapter discusses the development of the ACU based on the software simulation of the ACU (SoftACU) and battery management system (SoftBMS). The development process based on the soft electronic control unit simulation accelerated the development and reduced the risk of high voltage electric system failures. Because of the unique features of the 2-Mode hybrid electric vehicle, it presented some unique hazards in the integration, maintenance, service and repair. The high voltage battery and hybrid powertrain components may create a potential shock hazard. In the following discussion, the ACU functional requirements and interface are presented. The BMS function and control features are analyzed and simulated. The simulation was accomplished in a model with SoftACU, SoftBMS, multi-cell battery and electric distribution system (EDS), shown as Figure 7-1. The simulation results were validated in the application of the ACU in the 2-Mode HEV. 


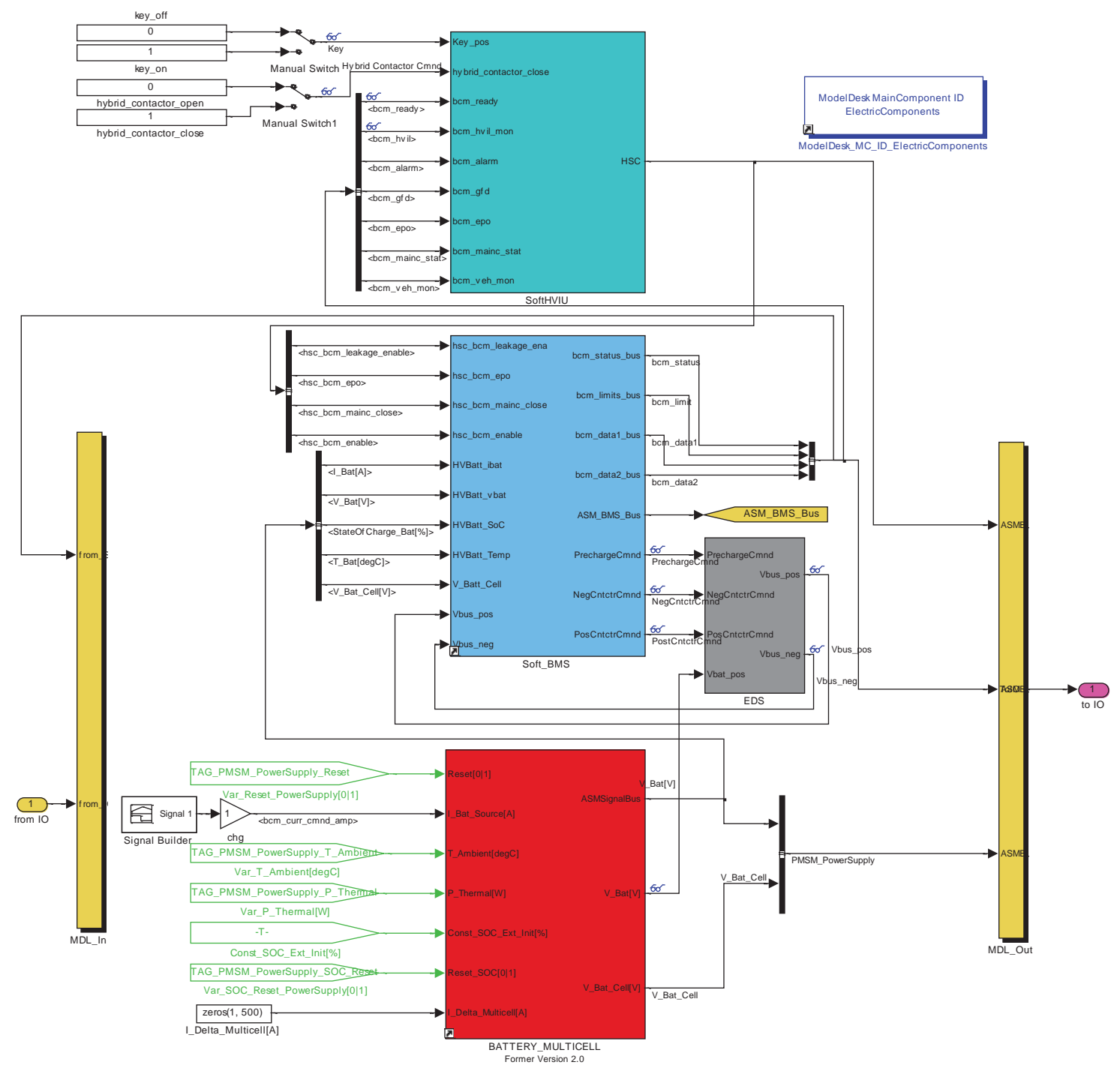

Figure 7-1: Model integration of high voltage system.

\subsection{1 $\quad$ Auxiliary Control Unit}

The ACU interfaced with the BMS for the control of high voltage power flow to the TPIM, shown in Figure 7-2. The ACU regulated the power flow between the high voltage battery and the TPIM. The ACU interfaced with the BMS and the vehicle control system through the control of the battery main contactors and pre-charge contactors. 


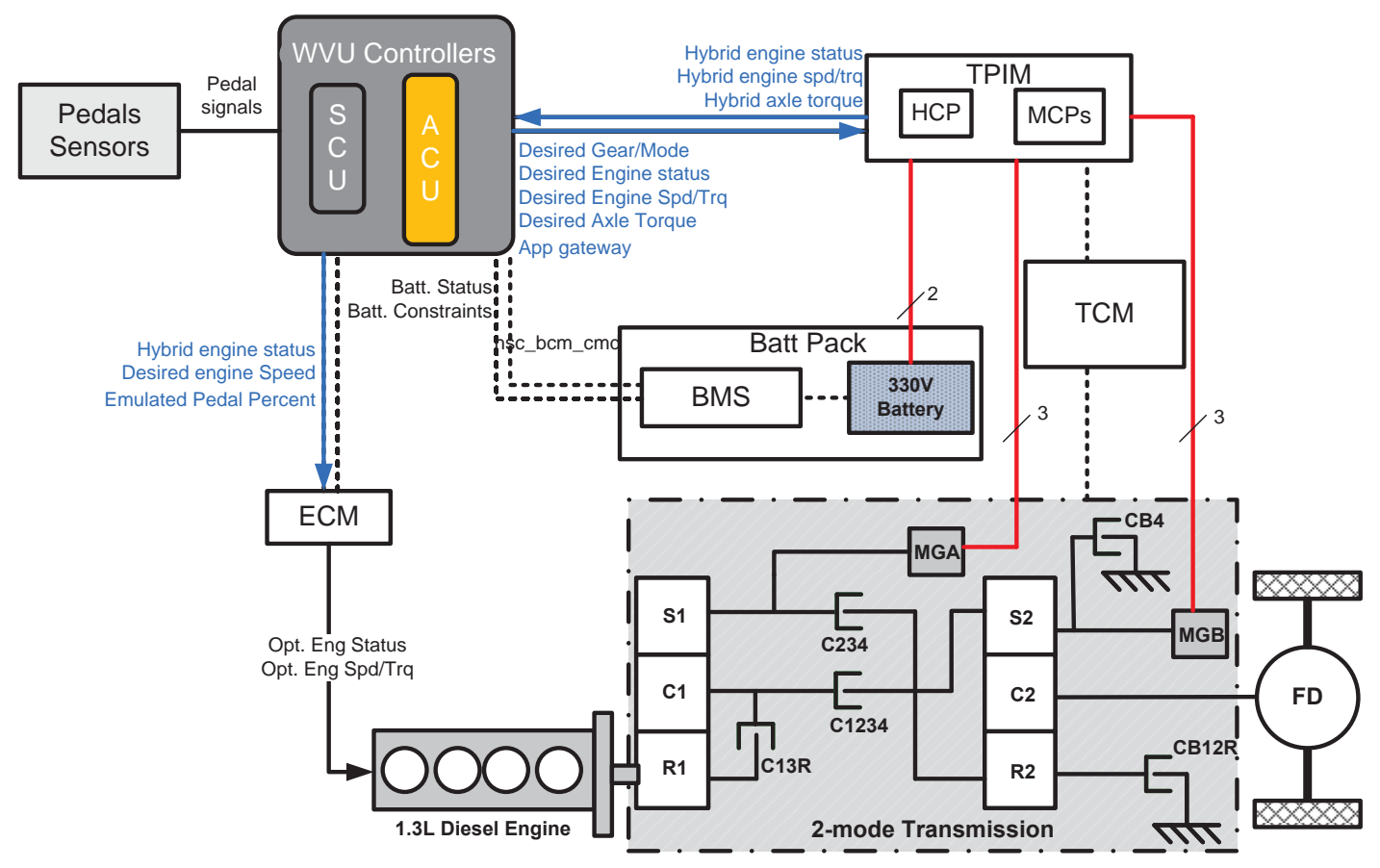

Figure 7-2: ACU interface in the 2-Mode HEV.

The ACU retrieved battery status from the BMS through CAN communication, shown in Figure 7-3. The ACU collected both the high voltage battery status from BMS and the vehicle status from SCU, DECM and TPIM to control of the high voltage enable signal, the close main contactor signal and also issue errors if there was any failure detected from the high voltage system. 


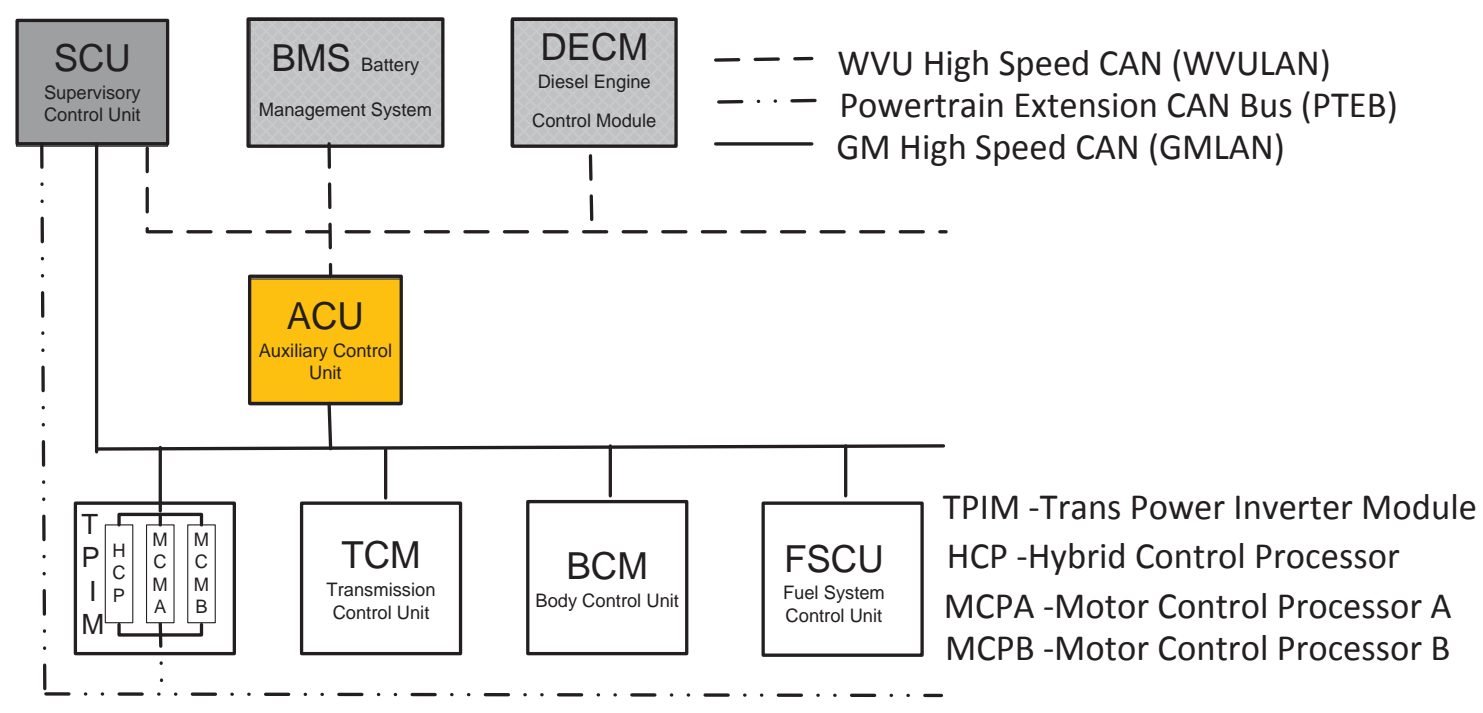

Figure 7-3: ACU CAN communication.

\subsubsection{Battery Management System}

Generally, the BMS in the HEV is designed to monitor, balance, and protect the battery pack. The BMS for a large multi-cell battery pack is responsible for monitoring the status and availability of the battery pack, protecting the battery pack and triggering data recording of the battery pack. The BMS is the data center of the battery pack that controls and monitors the battery pack to make sure the battery pack is operating correctly. The technology to manage the battery system for peak performance and high safety standards is complex, and requires state of the art design and testing to guarantee the best results. The BMS needed to address all of the needs associated with the complex high voltage battery pack, including safety, accuracy and compliance with HEV standards. A well designed BMS results in improved battery life, safety, scalability, decreased charge times, and advanced fuel gaging.

A typical BMS system integrated with the battery pack is shown in Figure 7-4. The BMS has variable digital and analogue I/Os to manage and communicate with 
various sensors and actuators as well as to interface with other electronic control units.

The BMS can support CAN network communications which are typically used for invehicle communication.

The module control board monitors the multiple battery cells connected in series or/and parallel within a battery module. The module control board senses cell voltage and temperature and reports these values to the BMS.

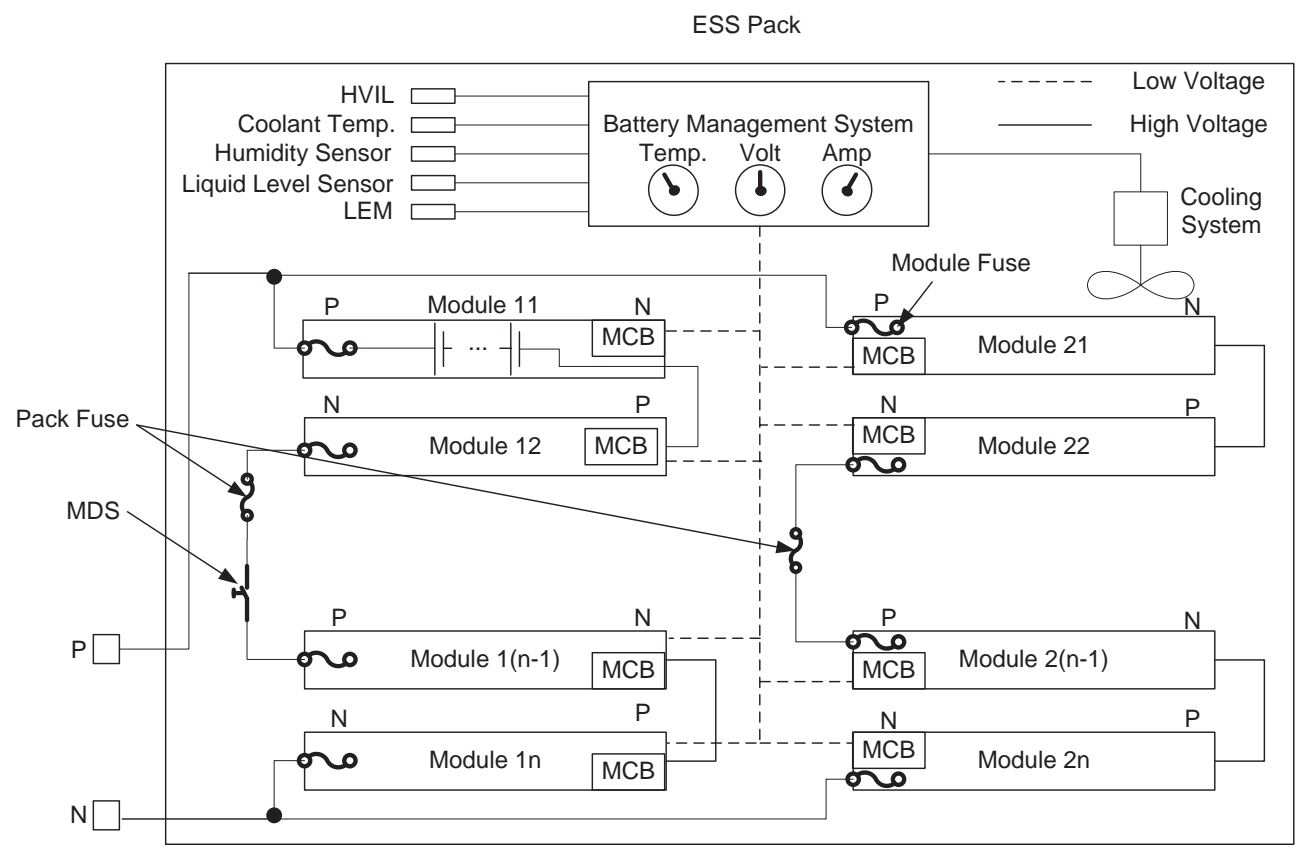

Figure 7-4: BMS and battery pack.

The BMS function varies based on different applications. Most commonly used functions include:

- $\quad$ SOC monitoring

- Stage of health monitoring

- Cell balancing 
- Cell failure detection

- Contactor control

- Pre-charge function

- Startup/shutdown

- Charger communication

- Thermal management

- Isolation detection

- HV-interlock

- Safety monitoring

- On-board diagnostics and service

- Error management

- CAN communication

The 2-Mode HEV BMS played important role to improve the vehicle performance. The BMS detected the important battery safety signals and the battery cell status. Communication between the ACU and BMS included the battery power status and safety status. In the power level status, the BMS was responsible for the information of the battery voltage, current, temperature, SOC and available discharge/charge power. In the safety level status, BMS provided the information of the battery temperature, emergency power off (EPO) status, battery alarm level and High Voltage Inter-Lock (HVIL) status. The BMS commanded the electric distribution system to control the main contactor to switch the high voltage line. 


\subsection{ACU Control Algorithm Development}

The BMS used in the vehicle had a normal startup sequence and a shutdown sequence in order to be securely integrated with the hybrid control system. The ACU needed to follow the requirements and sequences to make it work correctly. In the normal startup sequence, the BMS monitored the following signals in a sequence: ignition key>HVIL->HSC enable signal->contactors power check->pre-charge contactor check-> main contactors welder check-> close main contactors. The contactor power check was processed by enabling contactor power and confirming the contactor power relay closed status.

Before the high voltage system was powered up, it was important to know that the main contactors were properly functioning and in the expected open status. The main contactors may form a micro-weld when high current arcs across the contacts during opening or closing. Subsequently, when the main contactors were commanded to open, the poles would not separate and the high voltage system could not be shut down properly even though the main contactors had been switched to its open state electrically.

The normal startup sequence applied the above process to check the initial status and weld status of the main contactors. The initial status of the main contactors and weld check were processed by measuring the battery and bus side (positive and negative) voltages with combined logic. The pre-charge contactor was checked by closing the precharge contactor and confirming the battery voltage was equal to the bus positive voltage. Contactor weld status was checked by comparing the positive/negative bus voltage with the battery voltage after commanding close and open contactor. This methodology can 
perform the weld check and inform the BMS whether the contactor was closed at the initial status. This was an essential safety feature.

The BMS followed the normal shutdown sequence to open the high voltage battery main contactors. The BMS monitored the following signals in a sequence: ignition key-> HSC shutdown request-> wait for current ramp-down->main contactors welder check-> open main contactors. The wait for current ramp-down was to reduce the possible electric current arcing. The electrical current arc could occur when contactor transited from close to open with high current load. The electric current arc is typically more energetic, which may burn the contact's coating.

The hybrid system monitored the electrical potential between the high voltage and the vehicle chassis. High voltage should always be isolated from the vehicle chassis by a certain amount of resistance (typically $100 \mathrm{k} \Omega$ ) to avoid the potential for a life threatening current path. In the event that a high voltage leak path was detected to the vehicle chassis, the hybrid system set a diagnostic trouble code. Detection of a high voltage leak before vehicle start would prevent vehicle startup by disallowing the high voltage battery contactors to be closed by the hybrid control algorithm. However, a high voltage ground fault detection during the vehicle operation wouldn't open the main contacts immediately. But the hybrid control algorithm would prevent the vehicle from restarting again after it was stopped. The high voltage battery isolation status was monitored by both the TPIM and the BMS.

The control of the high voltage battery by the hybrid system included the vehicle status check, BMS initialization, fault detection, main contactor control and HV discharging/charging. The high voltage battery control algorithm is shown in Figure 7-5. 
The BMS was awakened by detecting the $12 \mathrm{~V}$ potential to the BMS wakeup pin. The BMS self-check was initialized at power up to check the power source to the main contactors. A fault trap was a special safety feature of the high voltage battery control. If an isolation fault was detected during the high voltage system activation, the control system was trapped in a fault status to prevent the main contactors from being closed unless the vehicle control system was reset or the isolation fault was removed. The HV discharge status ensured that HV current was reduced before it started to open the main contactors. To open the main contactor with high current load would shorten the main contactors service life or could form a welding of the contactors. A discharge timeout enabled shutdown to happen faster in response to the driver's command or an emergency situation. 


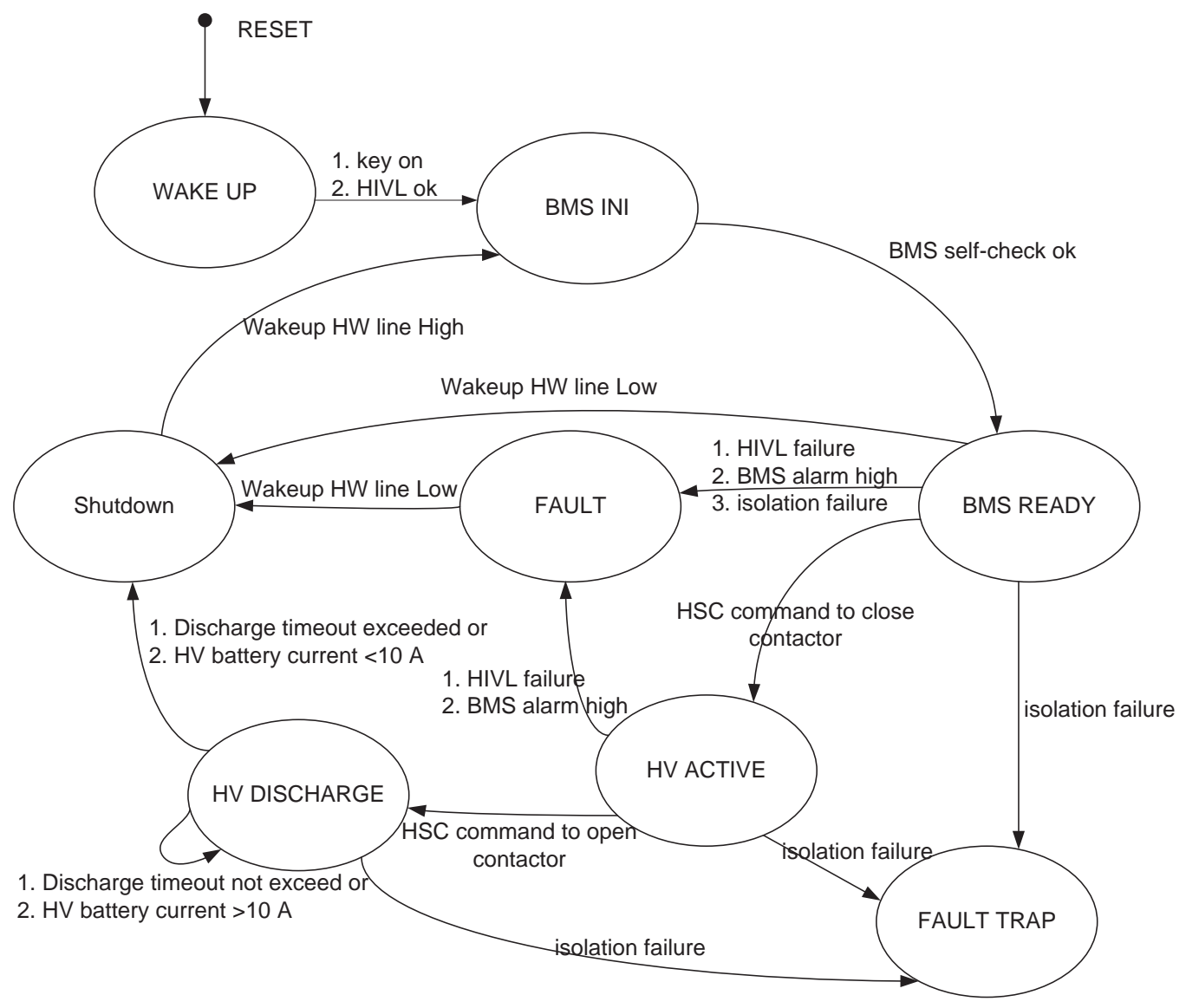

Figure 7-5: High voltage battery main contacts control algorithm.

\subsection{SoftBMS Development}

\subsection{1 $\quad \underline{\text { SoftBMS }}$}

The SoftBMS was the simulated BMS, which provided a safe test method to evaluate the safety features and the abuse performance of the battery control system. Sensor and actuator failures were simulated to check if the BMS would mitigate the hazards. Abuse tolerance of the high voltage battery system was tested with the SoftBMS, such as over temperature, over/under cell voltage and contactor failure. The 
mitigation control algorithm was tested to check the effect of reducing the potential hazards.

The SoftBMS transmitted the CAN messages indicating the high voltage battery status and received the command from the SoftACU to control the actuators. The main SoftBMS CAN messages included battery status, battery limits, battery cell information and battery safety information. The SoftBMS also bypassed the motor torque request from the SoftACU to the battery plant model, where the torque value was converted to a current request based on the electric motor specifications.

\subsubsection{SoftBMS architecture}

The simulated high voltage battery control system included models of a controller (SoftBMS), an Electric Distribution System (EDS) and a high voltage battery plant, shown in Figure 7-6. The SoftBMS received the command from the ACU to control the main contactors in the EDS. The battery plant supplied the power to the vehicle when the EDS main contactors were closed. The sensor model detected the battery status, including the voltage, current flow, state of charge, temperature, as well as the HVIL, resistance and vehicle awake and the charger awake signal. The high voltage system close loop status was monitored by the HVIL, which was a safety circuit to prevent high voltage hazards when unintended failures happened to the HV system. 




Figure 7-6: SoftBMS model.

\subsubsection{2 $\quad \underline{\text { SoftBMS Model }}$}

\subsection{Battery Status}

The main signals included in the battery status subsystem were battery alarm, EPO and battery ready. 
Battery EPO signal indicated that a fault occurred that prevented the BCM from performing its normal functions. The EPO included the occurrences of bad HVIL, BMS memory error, battery cell under voltage, battery cell over voltage, $12 \mathrm{~V}$ battery low voltage $(<9 \mathrm{~V})$, and battery pack over temperature $\left(>60{ }^{\circ} \mathrm{C}\right)$.

The battery ready signal indicated a complete self-check and ready to work status. The battery alarm signal indicated battery overall condition. The battery alarm signal had three output values indicating different status:

- TYPE 1: Check System, full capability available. No major failure of sensor signals

- $\quad$ TYPE 2: Limited operating strategy, Power Reduction. Battery pack high temperature (between $55^{\circ} \mathrm{C}$ and $60^{\circ} \mathrm{C}$ ) and isolation fault were included.

- TYPE 3: Major Fault, Shutdown. All the EPO situations were included.

\subsection{Battery Limits}

The battery limits subsystem included the battery maximum discharge/charge current, battery discharge/charge buffer and battery cell over/under voltage error, shown in Figure 9-20. The cell over/under voltage error was modeled by checking the multi-cell battery cell voltage with the cell voltage boundaries. 


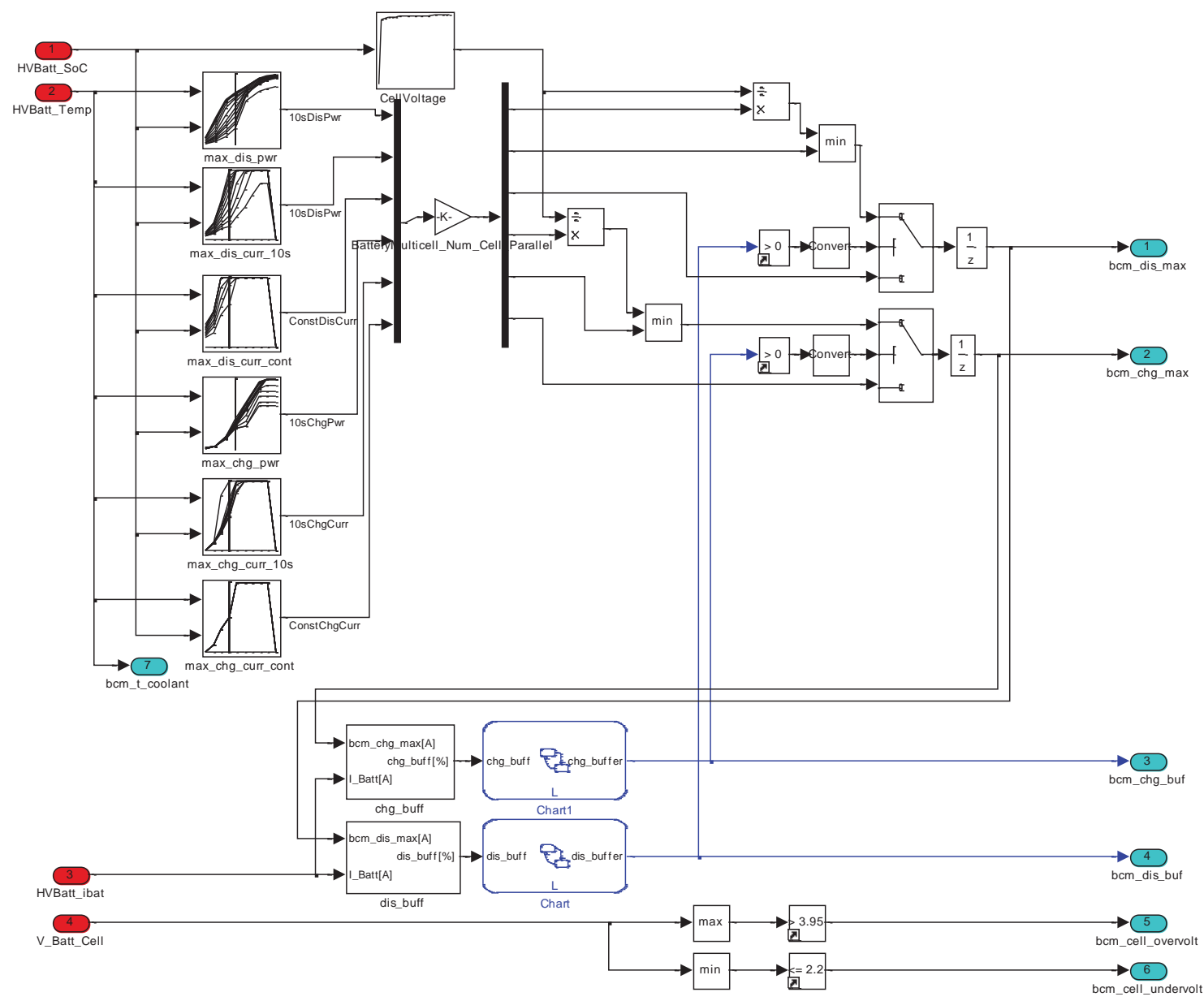

Figure 7-7: High voltage battery constraints model.

The battery maximum discharge/charge current was the maximum current rate the vehicle must stay within to prevent battery damage. The instantaneous maximum discharge/charge current was reduced as the cell transient capability was consumed, which was indicated by the battery discharge/charge buffer.

The battery discharge/charge buffer indicated how soon before the BMS needed to limit its maximum discharge current. The battery buffer was used by the SoftACU to manage the current reduction at a vehicle level. A 100\% battery buffer indicated a large buffer to accept discharge/charge up to battery maximum discharge/charge current. A 
$0 \%$ battery buffer indicated the battery maximum discharge/charge current being reduced to a steady-state level based on SOC and temperature. A 10-second discharge/charge current test data resulting from the hybrid pulse power characterization were implemented as 2-D lookup table in the model.

\subsection{Other Battery Information}

The BMS vehicle monitor signal indicated the vehicle wake status by checking whether the vehicle key position was in the crank/run position and the charger auxiliary was not enabled.

The BMS charger enable signal indicated the charger wake status by checking whether vehicle key position was off and charger wake signal was enabled.

The BMS cell max/min voltage indicated the battery cell maximum and minimum voltage by checking the multi-cell battery model voltage values.

Some sensor signals in the model were set as constants, such as BMS memory status, BMS flammable gas detection, $12 \mathrm{~V}$ battery voltage, HVIL status, isolation resistance value, BMS charger wake signal and BMS vehicle wake signal. Those constant values could be modified during the simulation to check the response of the SoftBMS and SoftACU to validate the control algorithms.

\subsection{Electric Distribution System Control Model}

An EDS control function was implemented in the SoftBMS to control the precharge contactor and the main contactors, shown in Figure 7-8. The EDS control logic followed the high voltage battery close/open main contactors procedure by checking the high voltage power status and the contactor welding status. 




Figure 7-8: EDS control algorithm.

\subsection{2 $\quad$ Electric Distribution System Plant Model}

The actuator model included the pre-charge and main contactors. The main contactors followed the EDS control command to energize the high voltage cable to power the vehicle, or to shut down the power supply. The main contactors were modeled as a switch to allow or disallow the battery voltage/current signals to pass from the battery model to the SoftBMS.

\subsubsection{Battery Sensor Model}

The sensor model simply provided the output of the BMS and high voltage battery information, such as HVIL status, ground fault status, battery alarm status, battery EPO status, battery main contactor status, battery pack voltage, battery current, temperature and state of charge.

\subsection{4 $\quad \underline{\text { SoftACU Model }}$}

The SCU communicated with the BMS to control the hybrid power from the battery. The SCU sent the command to wake up the BMS, which then returned the battery ready signal by checking the status of the high voltage electrical system. Upon 
receiving the battery ready signal from the BMS, the SCU sent the main contactor close command to close the main contactors and activate the high voltage power supply.

The SoftACU was built to implement the functions required for the in-vehicle application, as shown in Figure 7-9. The SoftACU read the SoftBMS messages and implemented the functions to command the high voltage battery contactor, enable high voltage battery isolation check, issue EPO warning and enable battery management system operation.

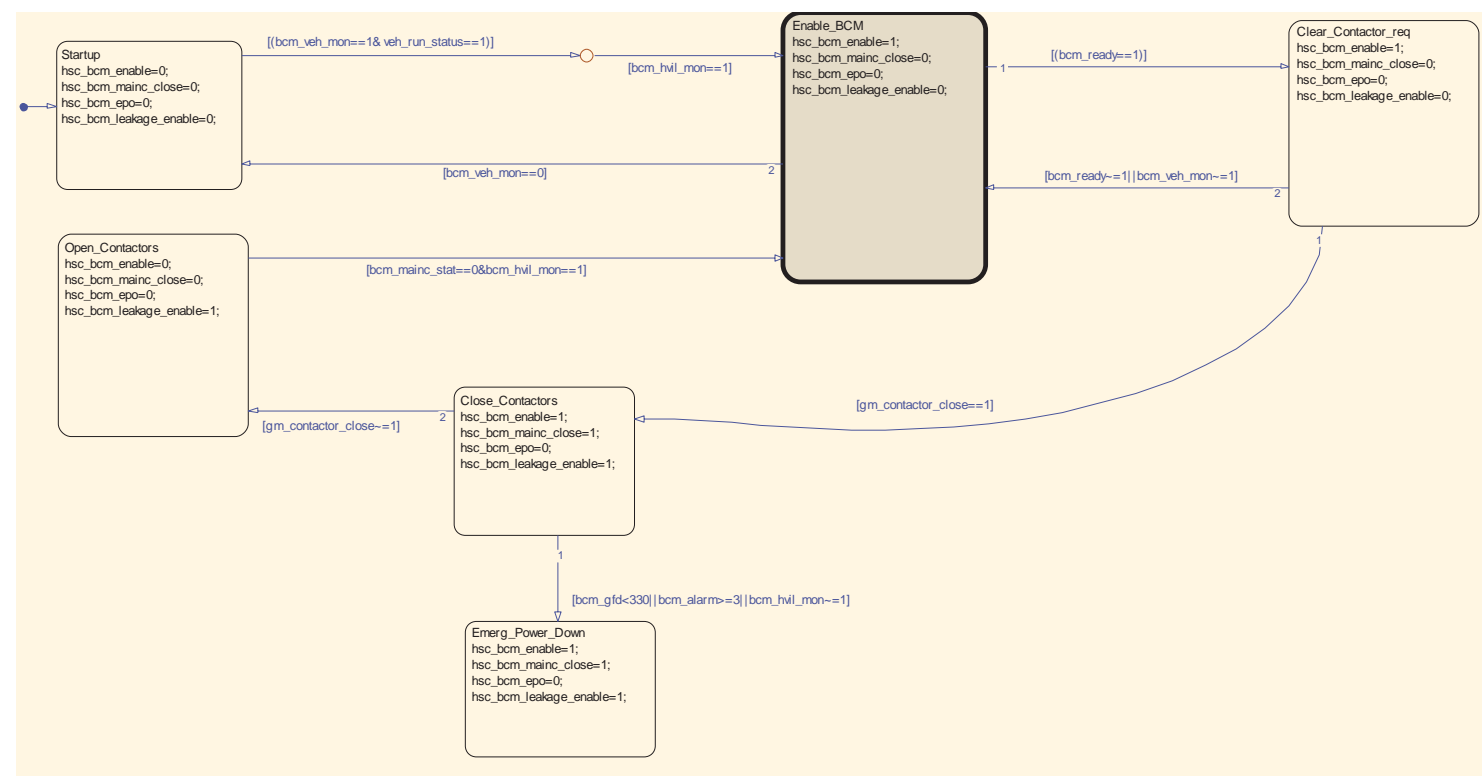

Figure 7-9: ACU control Stateflow.

The close high voltage main contactor was commanded by the hybrid controller. The BMS preceded execution of the hybrid main contactor command by initiating the bus pre-charge and main contactor closing/open commands to the EDS.

The high voltage current leakage detection enable signal was used to enable the ground fault detection. Leakage measurements were made on the battery bus. The vehicle emergency power off command was a fast shutdown request to open all 
contactors immediately, skipping any tests normally scheduled to occur during contactor open.

7.3.5 $\quad$ High Voltage Battery Model

The high voltage battery model is described in detail in CHAPTER 4 2-Mode Hybrid Electric Vehicle Model.

\subsection{Test Results and Summarization}

The integrated high voltage system was tested to validate the SoftACU control algorithms. The tests included normal procedure to close main contactors, normal procedure to open main contactors, the SoftACU response to HIL failure and isolation failure.

\subsection{1 $\quad$ Test SoftACU Control Commands}

The test result of the SoftACU control model in Figure 7-9 is shown in Figure 7-10. The SoftACU responded to the key signal and the hybrid contactor command to enable the SoftBMS, the leakage check, and to close the high voltage main contactors.

The test result of the ACU in-vehicle application validated the SoftACU control program, shown in Figure 7-11. The ACU responded to the key on signal to enable the BMS, enable the leakage check and close the high voltage main contactors. 


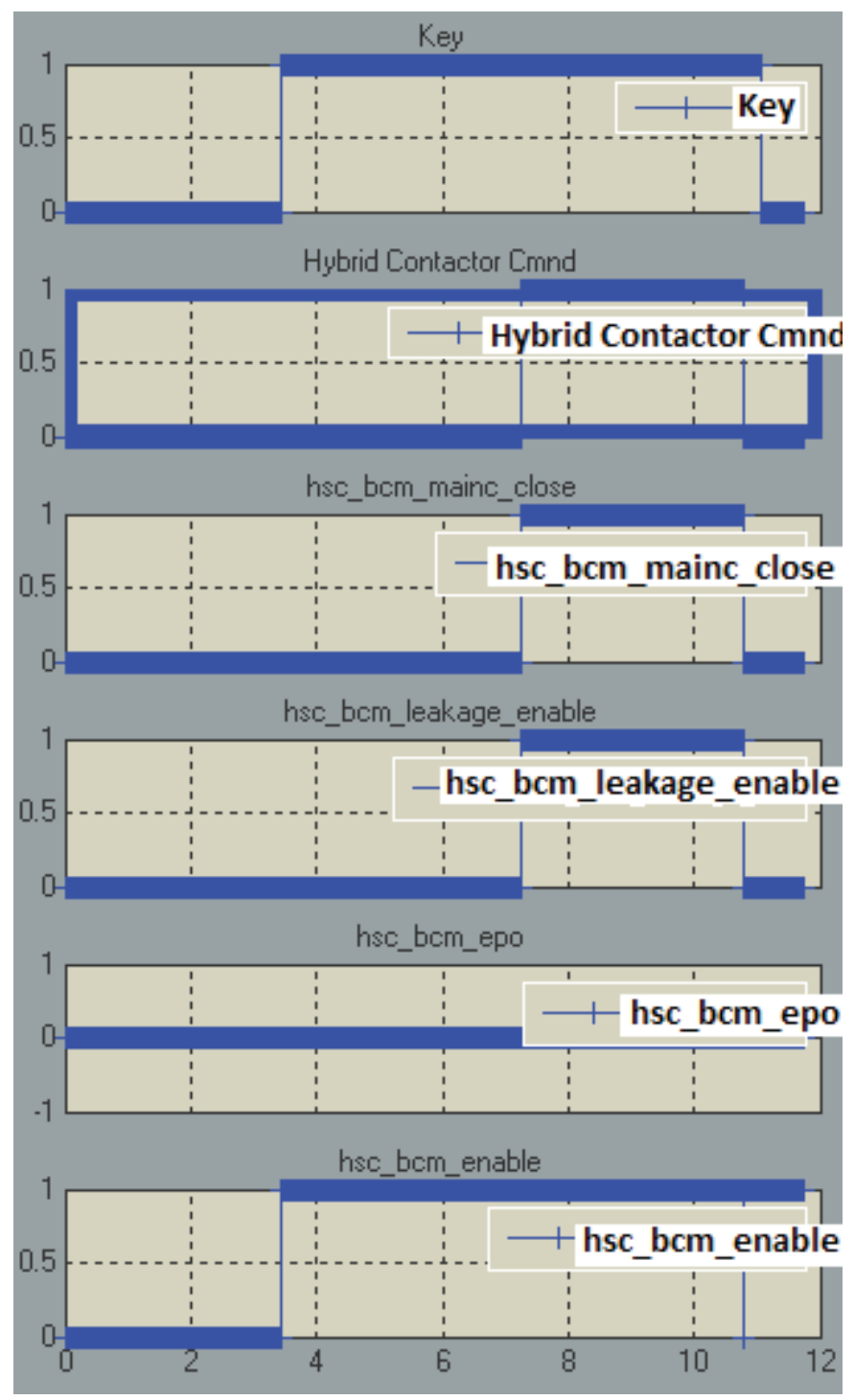

Figure 7-10: SoftACU simulation results. 


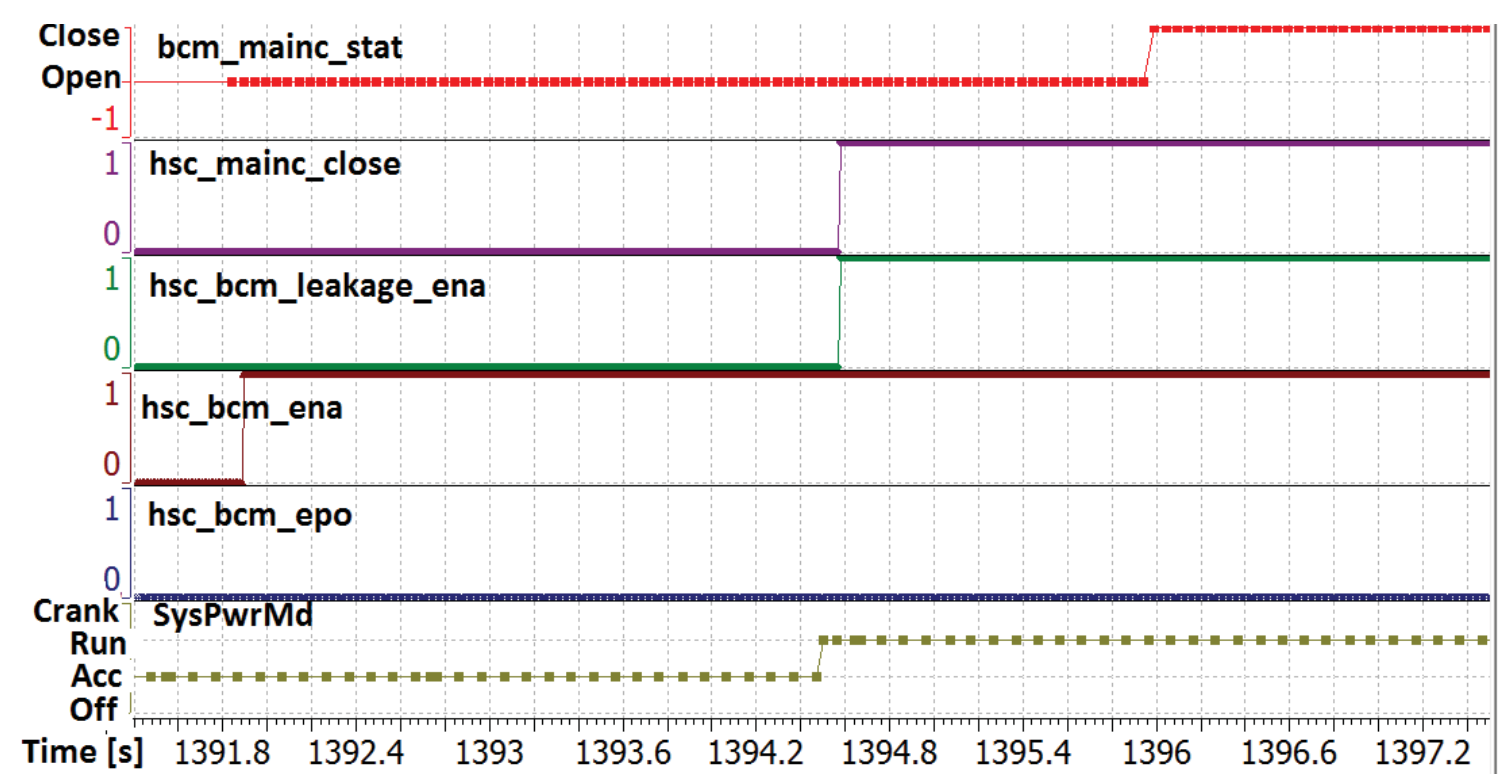

Figure 7-11: In-vehicle test of ACU control results.

\subsubsection{Test of the SoftACU to Close/Open the Main Contactors}

The SoftACU executed the key on signal and the hybrid contactor command to control the high voltage battery main contactor close/open, shown in Figure 7-12. The SoftACU executed the key on signal to enable the SoftBMS, which then enabled the contactor power relay monitor and read the contactor power relay command state. As the hybrid contactor command was detected, the SoftBMS engaged the main contactors and the high voltage positive bus was energized. The main contactors were open as the hybrid system controller commanded to cut the high voltage power connection. 


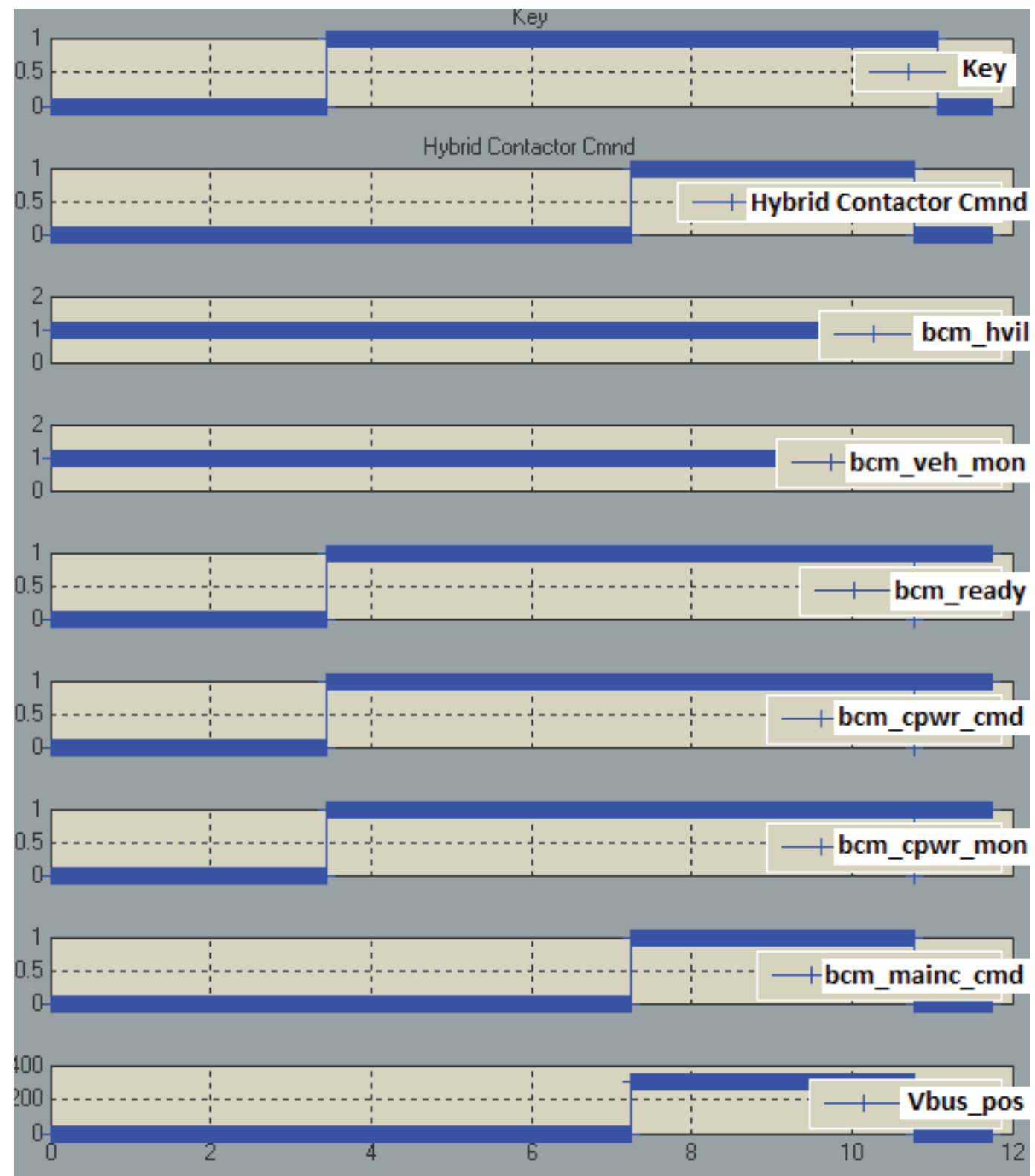

Figure 7-12: Test of SoftBMS open and close main contactor program.

\subsubsection{Test of the ACU Response to Sensor Failures}

The SoftACU control program was tested for safety concerns by activating fault conditions. The main contactors should not be closed if a ground fault appears before the vehicle starts, and should not open if ground fault appears during vehicle motion. The main contactors should not be closed if an HVIL fault appears before the vehicle starts, 
and should remain closed if a ground fault appears during vehicle driving. The main contactors should be open if the EPO status appears at any time.

- Ground fault detected before main contactor closed

The nominal high voltage resistance value (HV_Resistance [k $\Omega]$ ) was $1,100 \mathrm{k} \Omega$ when there was no ground fault. The ground fault status would be set when the HV_Resistance $[\mathrm{k} \Omega]$ was less than $330 \mathrm{k} \Omega$.

The main contactors were not closed as the ground fault appeared when the HV_Resistance [k $\Omega$ ] was set at $100 \mathrm{k} \Omega$, as shown in Figure 7-13.

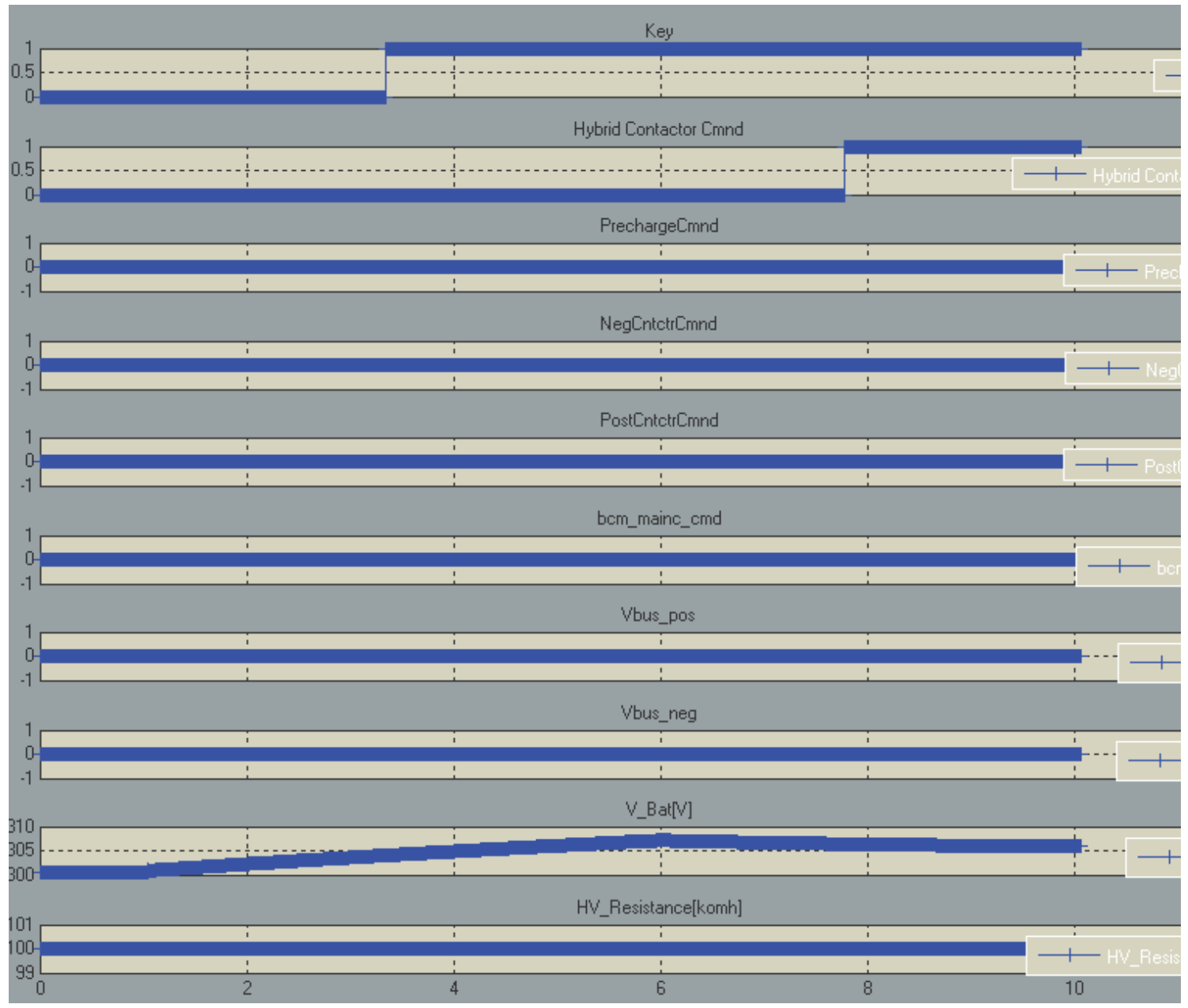

Figure 7-13: Ground fault detected before main contactor closed. 
- Ground fault detected after main contactor closed

The main contactors were not open as the ground fault appeared after the main contactors were closed, shown in Figure 7-14.

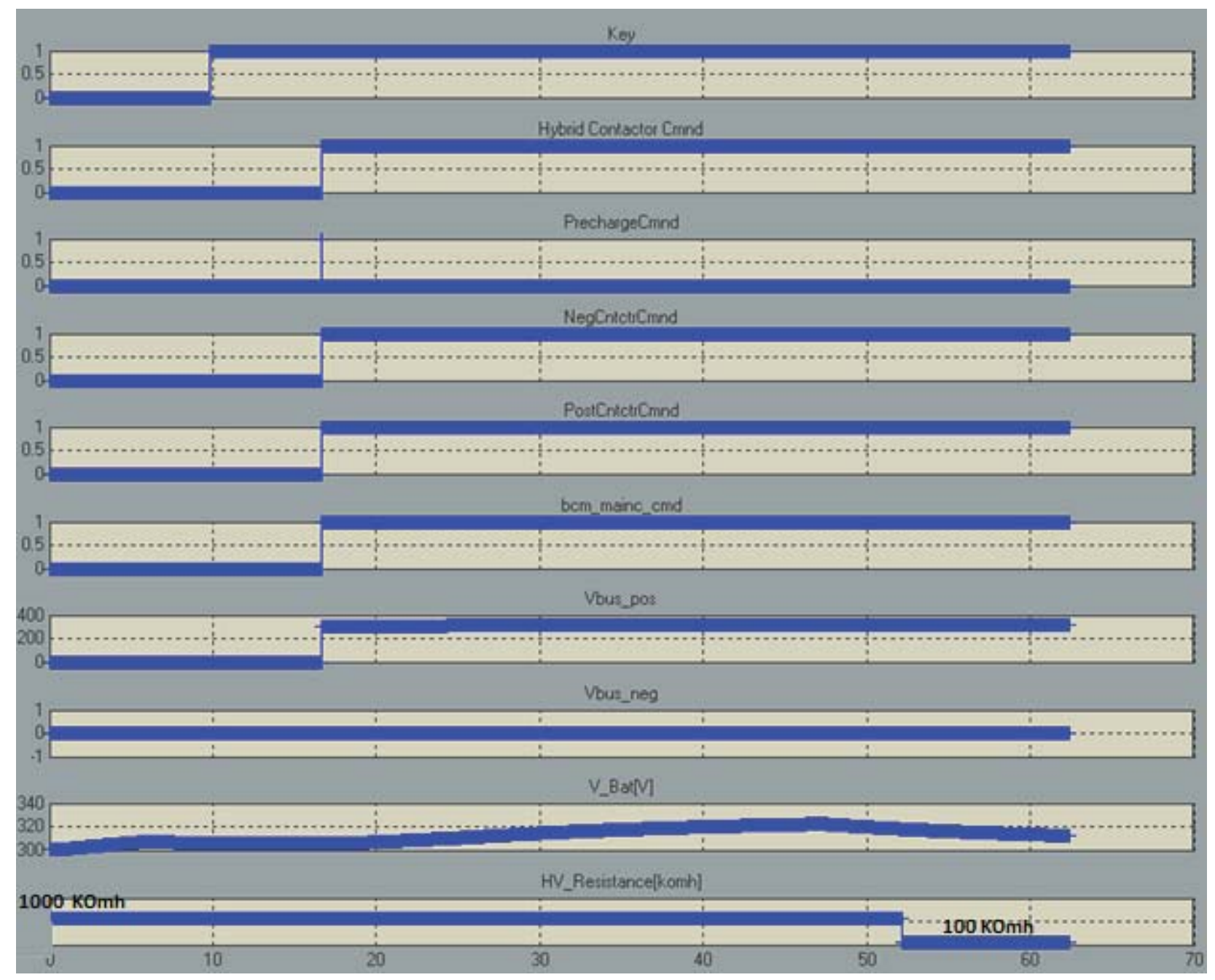

Figure 7-14: Ground fault detected after main contactor closed.

- HVIL failure before main contactor closed

The main contactors were not closed as the HVIL fault appeared, shown in Figure 7-15. 


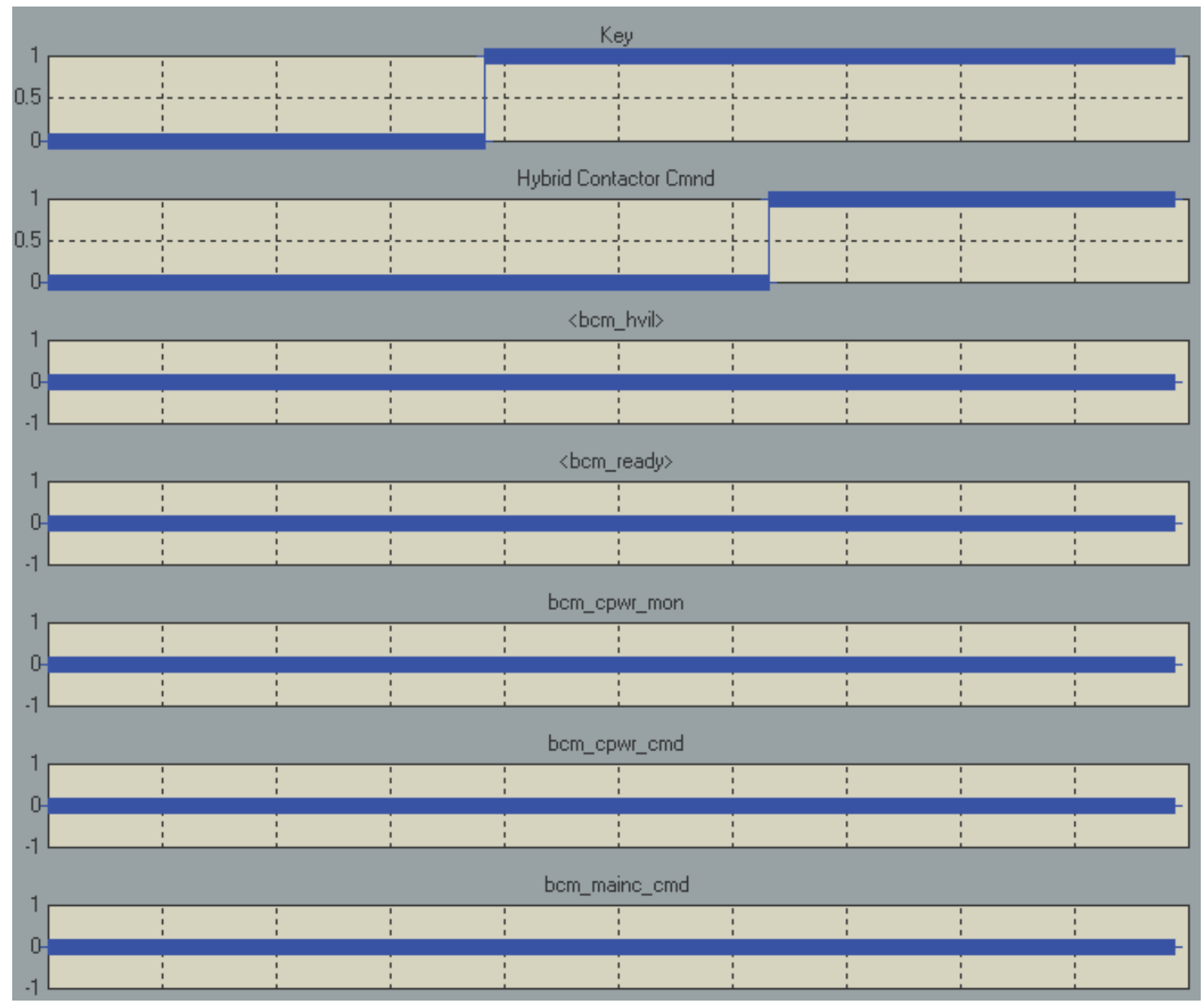

Figure 7-15: HVIL failure before main contactor closed.

- HVIL failure after main contactor closed

The main contactors were open as the HVIL fault appeared, shown in Figure 7-16. The HVIL was in the same circuit with the collision sensor. The HVIL fault was considered as severe as a collision, so the HVIL fault would open the main contactors as the vehicle was in motion. 


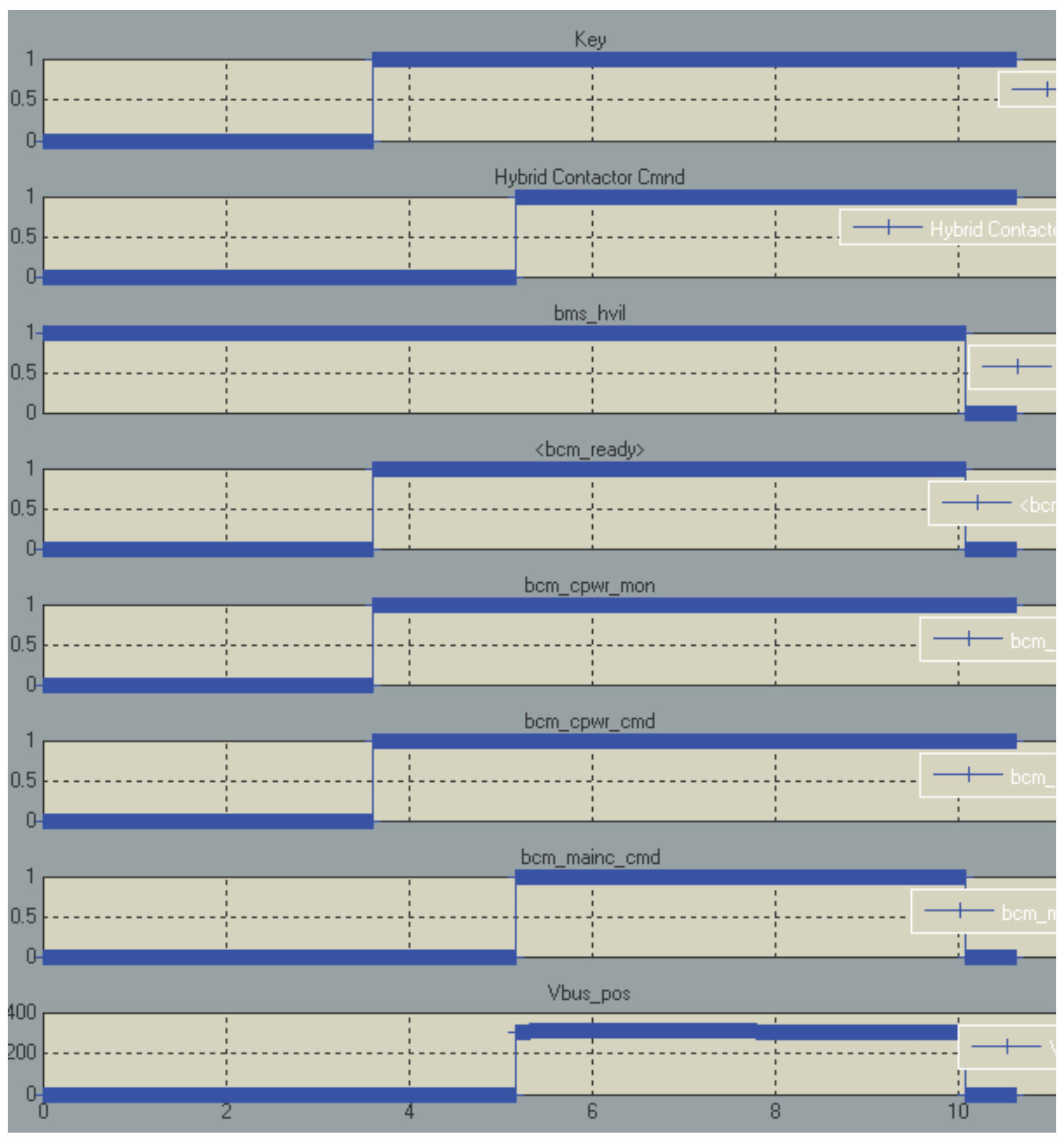

Figure 7-16: HVIL failure after main contactor closed.

- EPO failure after main contactor closed

The main contactors were open as the EPO fault appeared, shown in Figure 9-20 


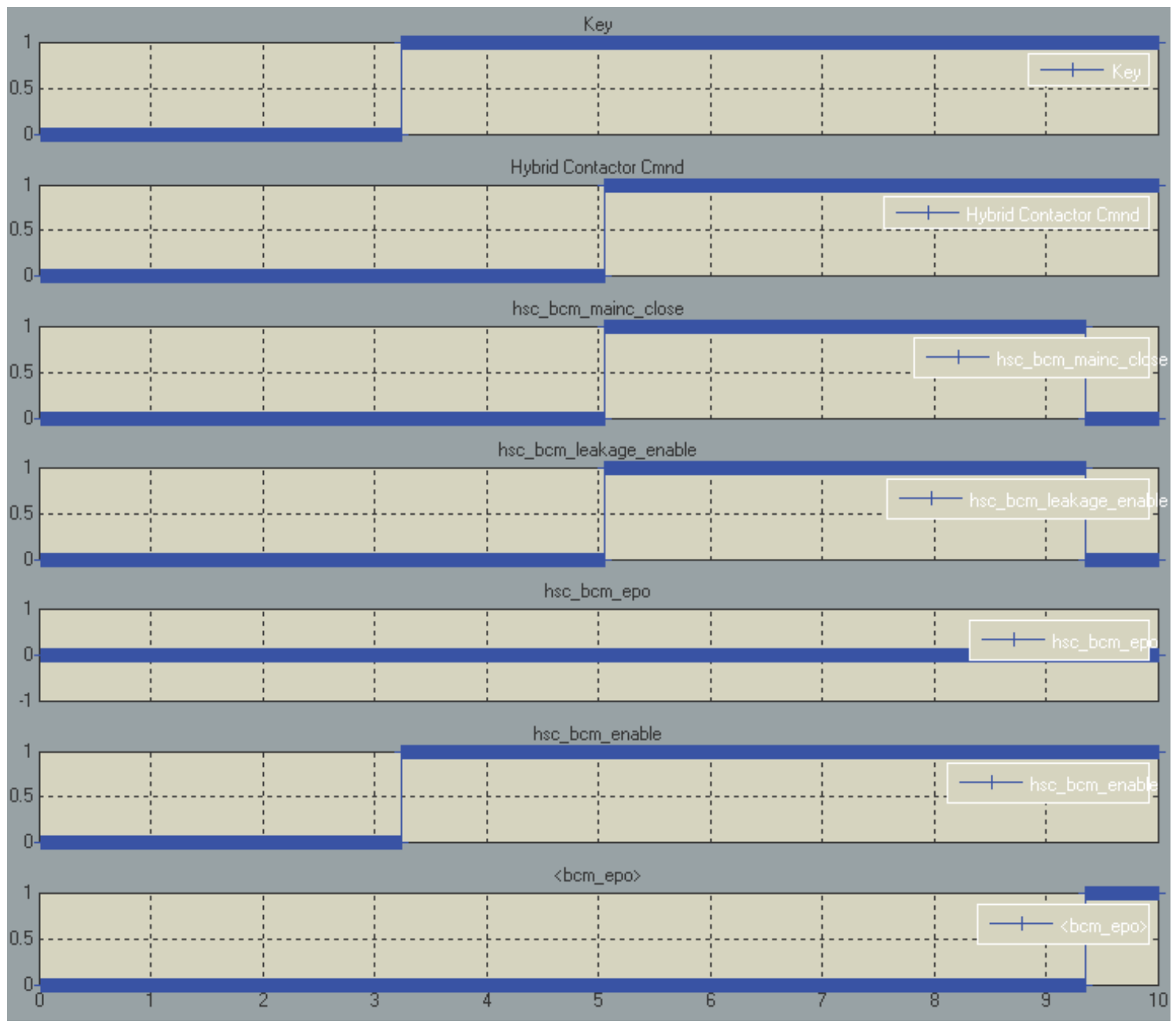

Figure 7-17: EPO failure after main contactor closed.

\subsection{Summary}

The simulation of the SoftACU in the Simulink environment proved to be a reliable method to develop the ACU control algorithm. The SoftACU was developed based on the hardware features and control requirements. The SoftACU was tested with the integration of the SoftBMS, hybrid system controller model and high voltage battery model. The test results validated the SoftACU control algorithm responding to the control command and fault status properly. 


\section{CHAPTER 8}

\section{2-MODE HYBRID CONTROL SYSTEM IMPLEMENTATION}

\subsection{Powertrain Integration Introduction}

\subsubsection{Engine Bay Integration}

Figure 8-1 shows the engine bay powertrain integration in the vehicle. The $1.3 \mathrm{~L}$ SDE diesel engine was mounted transversely in the passenger side of the engine bay. The 2-Mode transmission was connected to the engine with a custom-machined 6061 aluminum adapter. The transmission was located to the right side of the engine, under the TPIM. The TPIM converted the $330 \mathrm{~V}$ high voltage DC electrical energy to $275 \mathrm{~V} 3$ phase AC electrical energy to drive the two electric motors in the transmission. The TPIM was connected to each pole of the high voltage battery. Both of the negative and positive high voltage poles were isolated from the vehicle chassis by the resistance over $100 \mathrm{k} \Omega$. The electric Air Conditioning (A/C) compressor high voltage DC cables were externally connected at the TPIM.

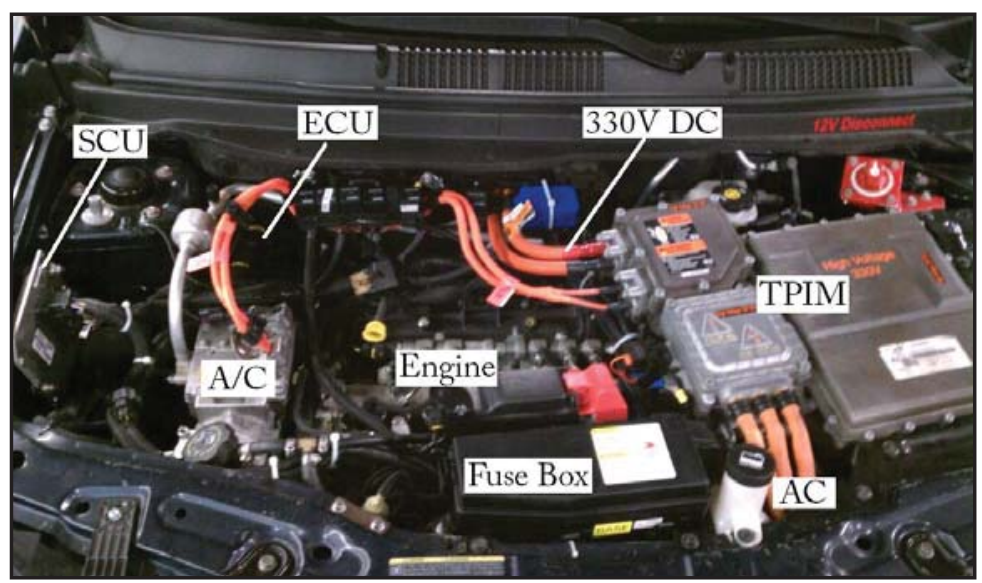

Figure 8-1: Powertrain integration. 


\subsubsection{High Voltage Battery Integration}

The high voltage battery was located in the rear cargo area of the vehicle below the load floor. The four battery modules were liquid-cooled via two aluminum cooling plates, which were sandwiched between modules as shown in Figure 8-2. The BMS controlled battery charging and discharging processes and communicated the battery status to the vehicle through CAN messages. The BMS was located inside the battery enclosure behind the "BMS" label in the photograph. The EDS housed the high voltage contactors, pre-charge contactor and hall-effect current sensors. The EDS was located inside the battery enclosure behind the battery connectors. The three high voltage battery connectors were color-coded "Charger" in orange, "Vehicle" in grey and "Mid-Pack Disconnect" in blue. Disconnecting the mid-pack connector reduced the battery pack voltage by one-half for increased safety during maintenance.

Two high voltage charger contactors were integrated between the high voltage battery and the TPIM on both the positive and negative cables. When the BMS was activated and in "Vehicle" mode, the high voltage charger contactors were closed to supply power to the TPIM to drive the electric motors. When the BMS was activated in "Charging" mode, the high voltage charger contactors were open to separate the TPIM from and the high voltage battery. The ACU was installed to the right under an interior panel. 


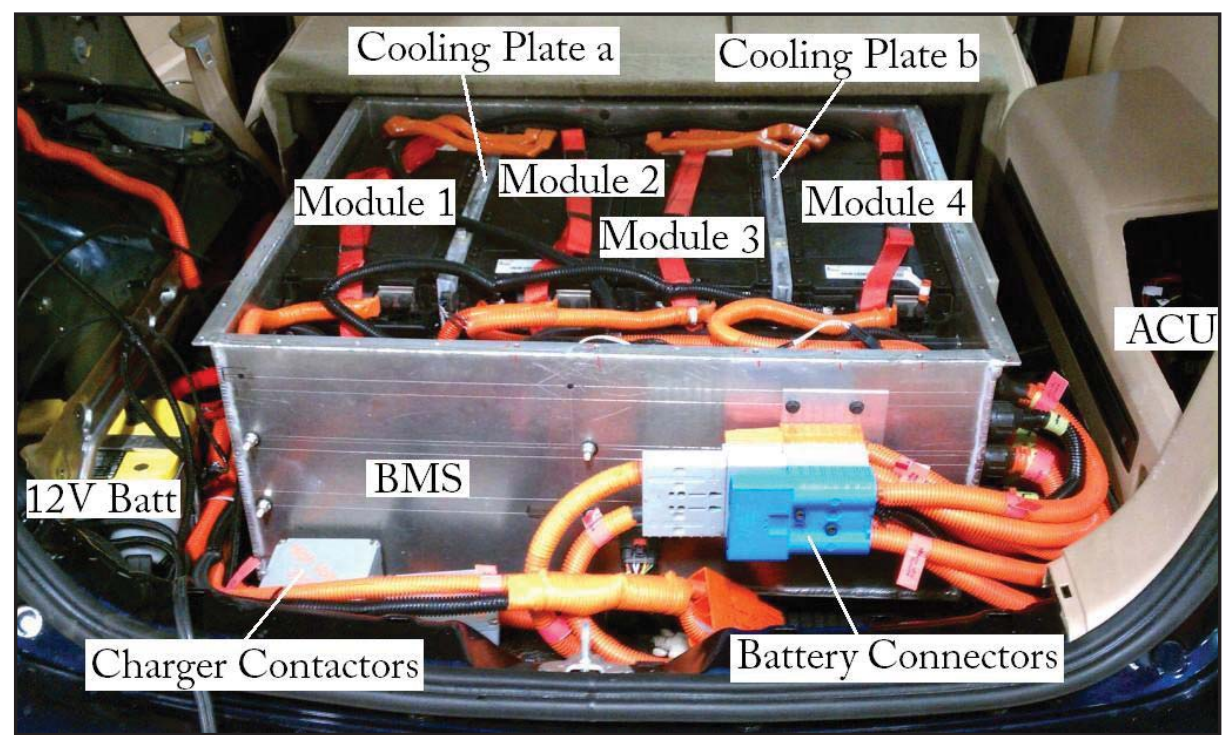

Figure 8-2: High voltage battery integration.

\subsection{Distributed Hybrid Vehicle Control System}

The 2-Mode hybrid vehicle controller distributed architecture is shown in Figure 8-3. A CAN made the use of a distributed control system possible in the vehicle. Several electronic control units could be placed around the vehicle to collect data from analog sensors and provide control outputs locally. Two controllers were added to the stock vehicle control system: the SCU and the ACU. 


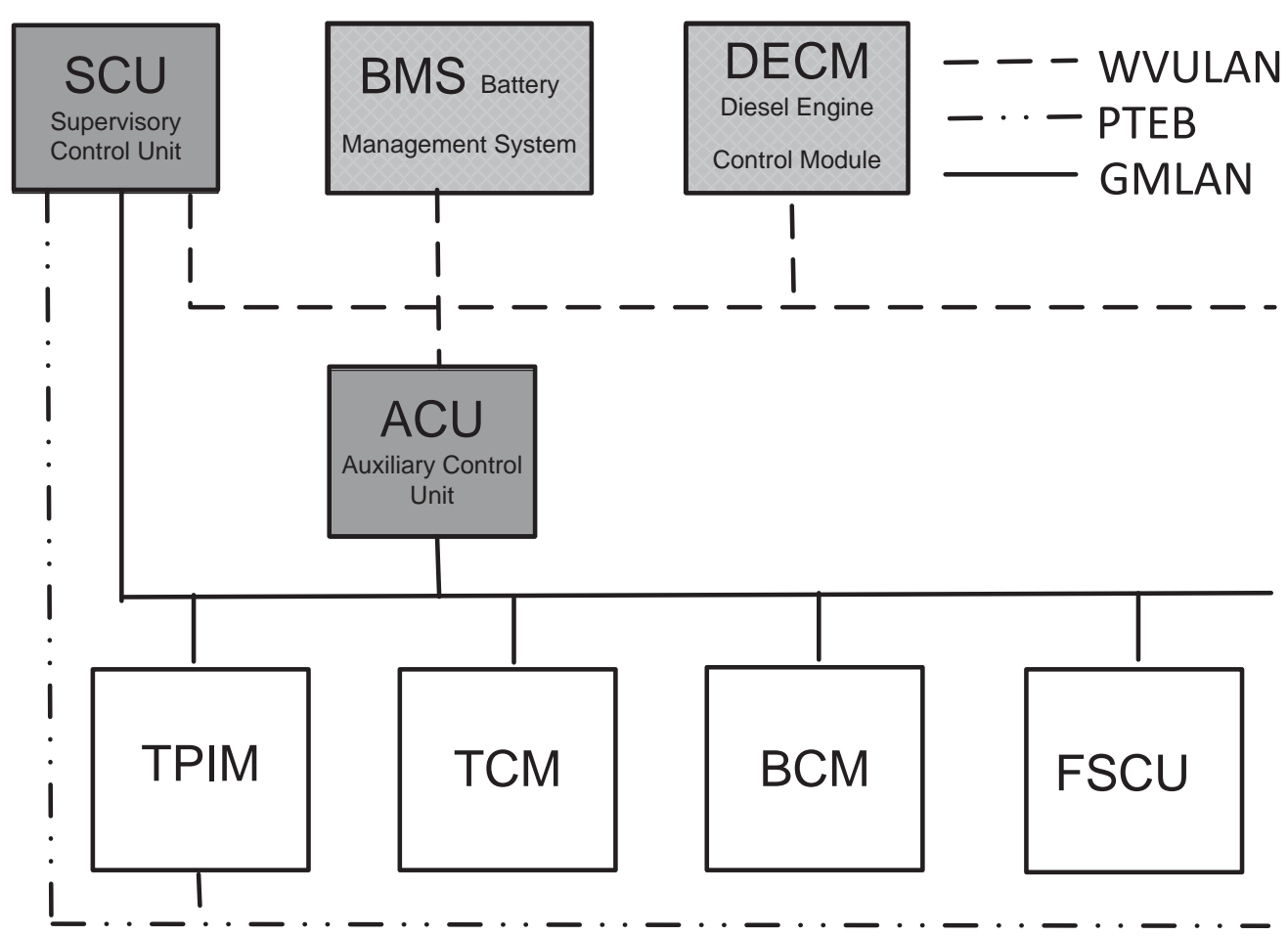

Figure 8-3: Distributed hybrid vehicle control system.

There were two existing CAN high speed buses in the vehicle: GM High Speed CAN (GMLAN) and Powertrain Extension Bus (PTEB). A third high speed CAN bus, WVULAN, was developed to integrate the BMS CAN communication and the DECM communication.

The main powertrain control CAN signals are shown in Figure 8-4. The SCU read physical APP signals and the vehicle speed to command engine stop-start, engine speed, engine torque and axle torque. The SCU also transmitted the physical APP signal to the TPIM through CAN communication.

The HCP played a role of protector in the hybrid control system. The HCP filtered the engine torque command, engine stop-start command and axle torque command from the SCU by checking the constraints of the electric motor from Motor Control Processor 
A (MCPA) and Motor Control Processor B (MCPB). The SCU then applied the feedback signals to command the engine stop-start, engine speed and engine torque. The DECM transmitted the actual engine stop-start status, actual engine speed and engine torque via CAN bus to the HCP.

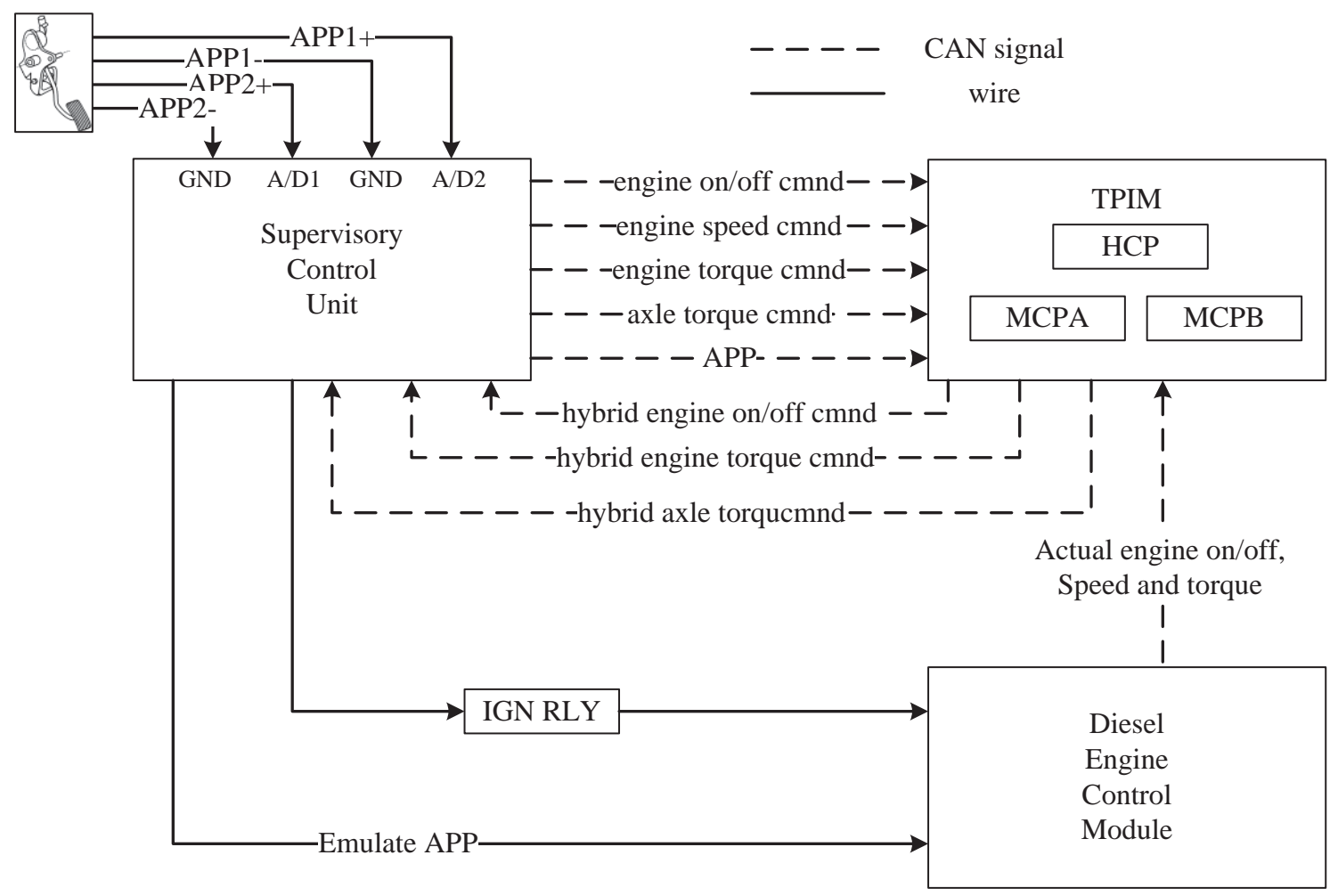

Figure 8-4: 2-Mode key powertrain control CAN signals.

\subsubsection{Supervisory Control Unit}

The SCU was the main hybrid system controller. It executed the strategic selection and tactical selection algorithms. The SCU determined the overall torque to propel the vehicle, as well as the regenerative braking power to charge the battery. It regulated the power ratio between the engine and the high voltage battery to propel the vehicle. The SCU determined when to perform hybrid operation modes such as engine 
stop-start, regenerative braking and transmission operation in EVT Mode 1 and EVT Mode 2. The SCU operated in conjunction with the BMS to determine when to enable and disable the DC high voltage circuits.

\subsection{2 $\quad$ Auxiliary Control Unit}

The ACU managed the high voltage battery power connection to the TPIM through the control of the battery main contactors and pre-charge contactors.

\subsubsection{Transmission Power Inverter Module}

The TPIM converted high voltage DC electrical energy to 3-phase AC electrical energy. The TPIM included a HCP and two motor control modules. The TPIM coordinated with the SCU to achieve the designated hybrid functions. The TPIM read the powertrain hybrid control commands from the SCU and fed back the corresponding hybrid commands by filtering out irrational commands that were beyond the powertrain capability based on current status. Two motor control modules controlled M/G1 and M/G2 respectively. Each motor control module operated the applicable electric motor generator based upon HCP commands.

\subsubsection{Transmission Control Module}

Changing gear ratios was fully automatic and was accomplished through the use of a TCM located inside the transmission. The TCM commanded shift solenoids and variable bleed pressure control solenoids to control shift timing and feel. All the solenoids, including the TCM, were packaged into a self-contained control solenoid valve assembly. 


\subsubsection{Diesel Engine Control Module}

The DECM determined the amount of fuel, ignition timing and other parameters of the internal combustion engine. The DECM read the engine sensors signals, such as the crankshaft position sensor, common rail fuel pressure, intake air mass flow sensor, accelerator pedal position and engine temperature to control the engine actuators, such as fuel injectors. For the 2-Mode hybrid electric vehicle, the main control function of the DECM was to achieve engine stop-start, and execute the optimal engine speed and torque.

\subsubsection{Battery Management System}

The BMS played an import role in the process of integrating the high voltage system in the HEV. The details of a typical BMS were introduced in section 7.1.2.

\subsection{Hybrid Powertrain Control Integration}

\subsection{1 $\quad \underline{\text { Real-time System Controller Introduction }}$}

The vehicle control system consisted of multiple electronic control modules connected with CAN bus. The distributed architecture of the vehicular control system supported the real-time applications. Tasks and control signals running on the real-time control modules were designed with run-time based priority. Different control modules interacted with each other using the connecting buses periodically with asynchronous communication, where all interacting tasks and signals were activated periodically and communicated by means of asynchronous buffers in the control modules.

\subsection{2 $\quad$ Accelerator Pedal Integration}

APP was one of the most important input to command vehicle driving power. In a traditional vehicle, the APP signal is directly wired to the engine to command engine 
power. However, in the 2-Mode hybrid vehicle, the APP signal was intercepted by the SCU. The APP sensor used two potentiometer signals to detect the accelerator pedal position.

The APP sensor was wired as shown in Figure 8-5. The SCU used two Analogue Digital Converter (ADC) channels to read the APP signals. The APP signals were sent to the DECM for engine torque command. The SCU Low Side Out (LSO) Pulse Width Modulation (PWM) signals were converted to analog outputs and sent to the DECM.

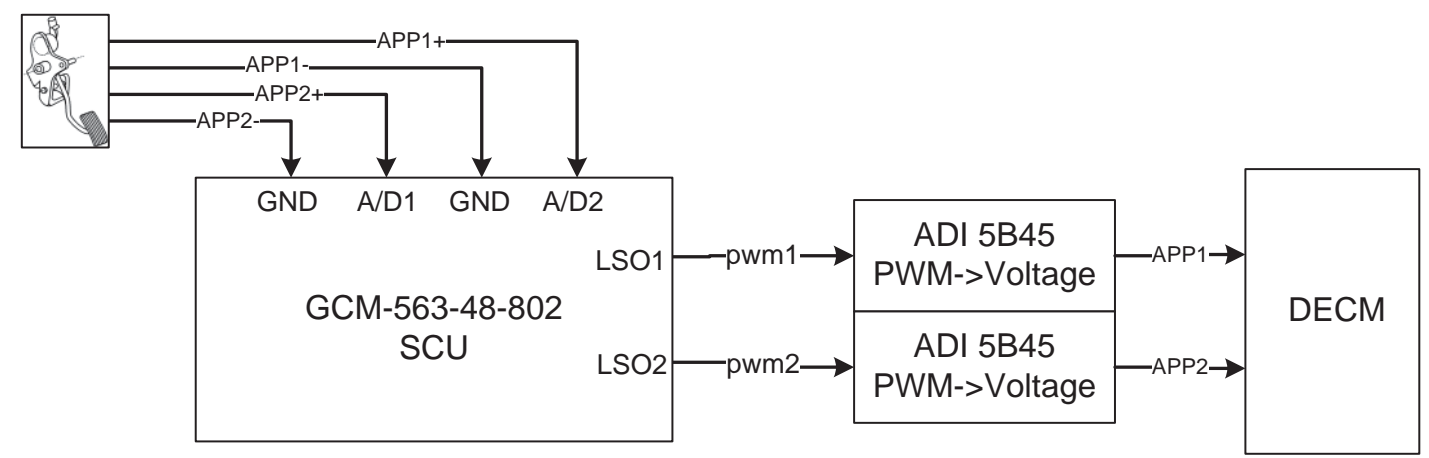

Figure 8-5: APP sensor wiring.

An APP signal validation algorithm was implemented in the SCU to prevent unintended vehicle acceleration from accelerator pedal signal failure, shown in Figure 8-6. The two APP potentiometer sensors were designed in the way that one sensor output was half of the voltage of the other one. A $10 \%$ tolerance of the two APP signals was set to allow for signal noise. If the offset was between $10-30 \%$, the vehicle was set to "limp home mode", which limited the maximum axle torque command to $30 \%$ of the normal maximum value in order that the vehicle was still drivable for a repair. 


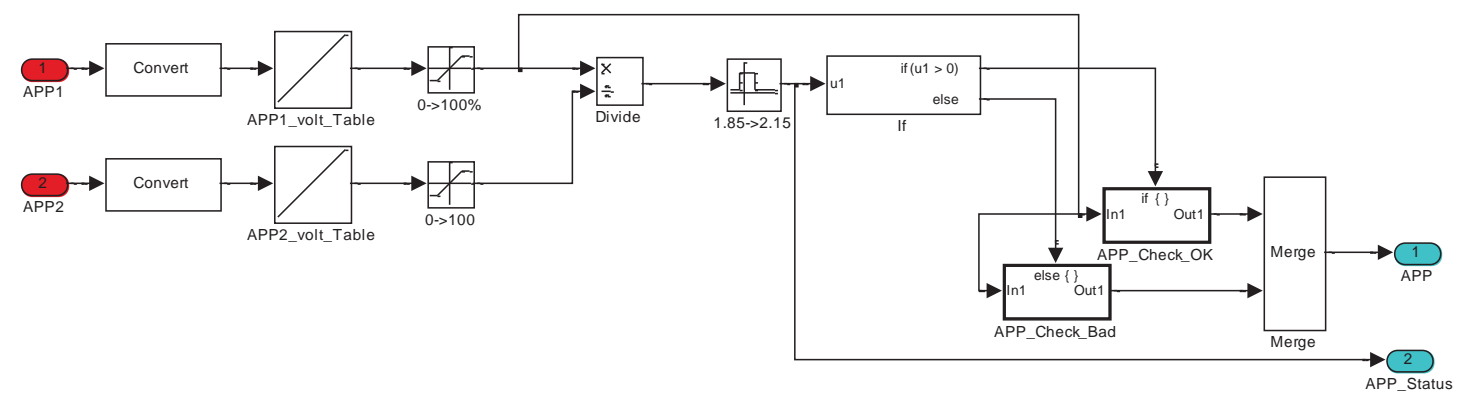

Figure 8-6: APP validation control.

The final engine torque was commanded by the TPIM as hybrid engine torque command, shown in Figure 8-4. A 2-D lookup table (LUT) was used to convert the engine speed and engine torque command to the accelerator pedal position, shown in Figure 8-7. Finally the pedal position PWM signals were generated by two LSO channels, which were then converted to analogue voltages by Analog Device Inc. 5B45 PWM to analogue signal converters. The conversion of the engine torque control command to the analog signals was necessary because the DECM could not respond to the torque requests via CAN interface between the TPIM and DECM. Analog Device Inc. 5B45 is a signal conditioner that generates output of 0-5 V proportional to isolated PWM input [78]. 


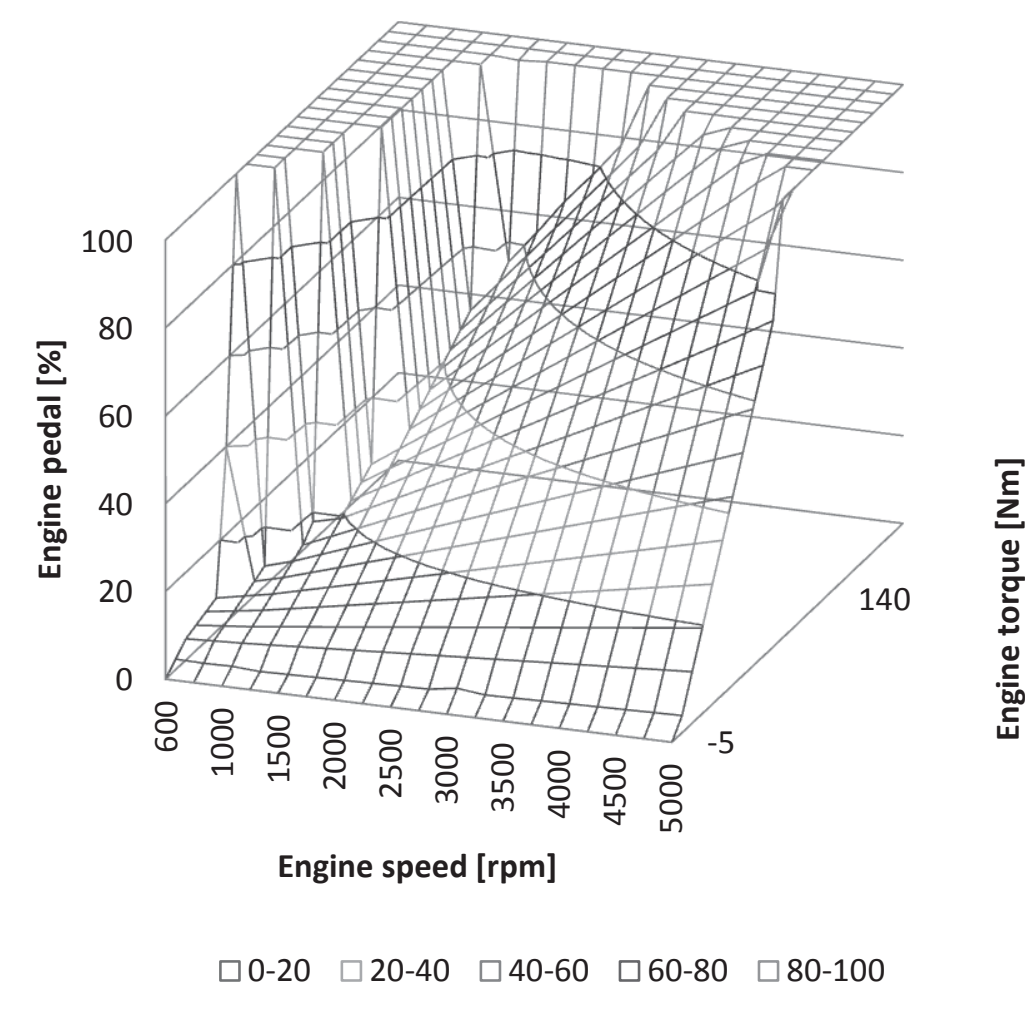

Figure 8-7: Engine pedal emulation.

\subsubsection{Engine Ignition and Stop-start Control}

The 2-Mode stop-start function was implemented by controlling the diesel engine ignition directly, shown in Figure 8-8. The ignition (IGN) pin on the DECM was guarded by the Run Crank Relay and the HVIL enable ignition relay (HVIL ENA IGN RLY). The Run Crank Relay detected the key position and the HVIL ENA IGN RLY detected the HVIL circuit status. The engine ignition can only be turned on when the key was in run/crank position and the high voltage interlock signal was correct. The IGN RLY signal was controlled by the SCU hybrid engine stop-start control command through the LSO channel. 


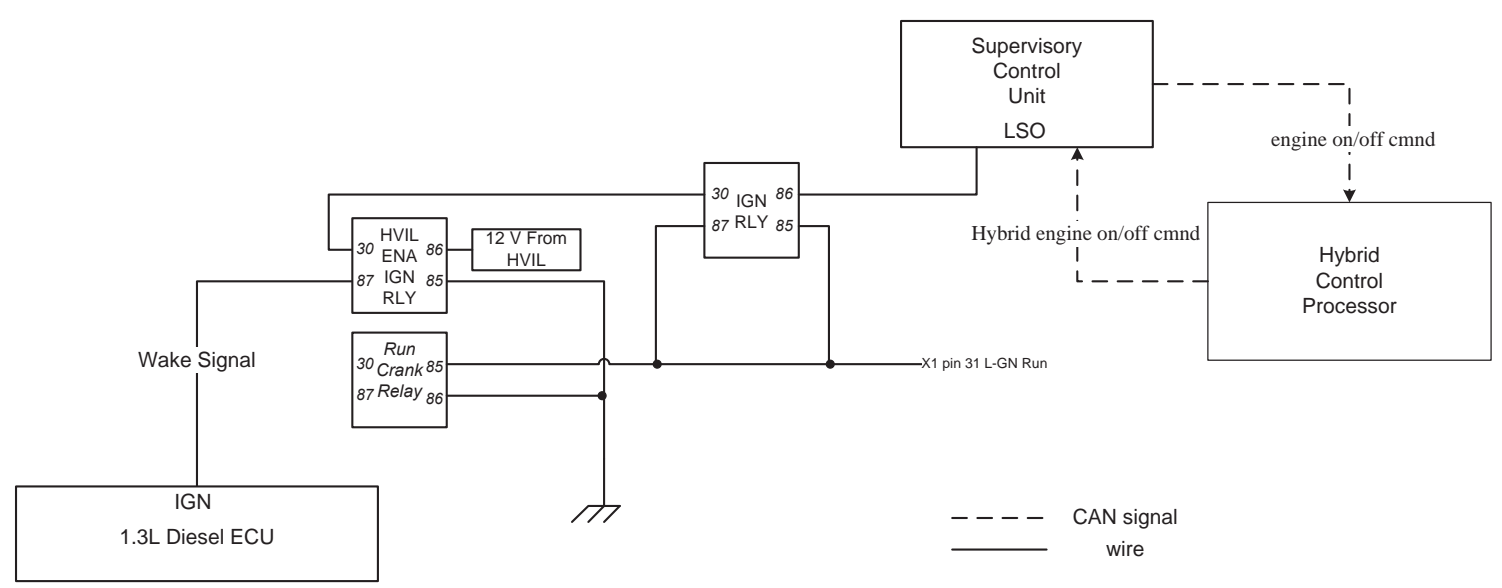

Figure 8-8: Engine ignition control.

\subsection{4 $\quad$ Hybrid Fueling System}

The traditional fueling system was modified to accomplish the special requirements of the hybrid system, shown in Figure 8-9. The fueling pump only turned on when the engine ignition was on. With any failures related to the high voltage system and the collision, the fueling system would stop pumping fuel to the engine. The fueling pump relay signal was guarded by the HVIL signal and the HVIL enable ignition relay (HVIL ENA IGN RLY) signal. Any failure of the HVIL would cause the fueling system to stop working. The fueling system was also deactivated by the ignition signal to achieve the engine stop-start function. 


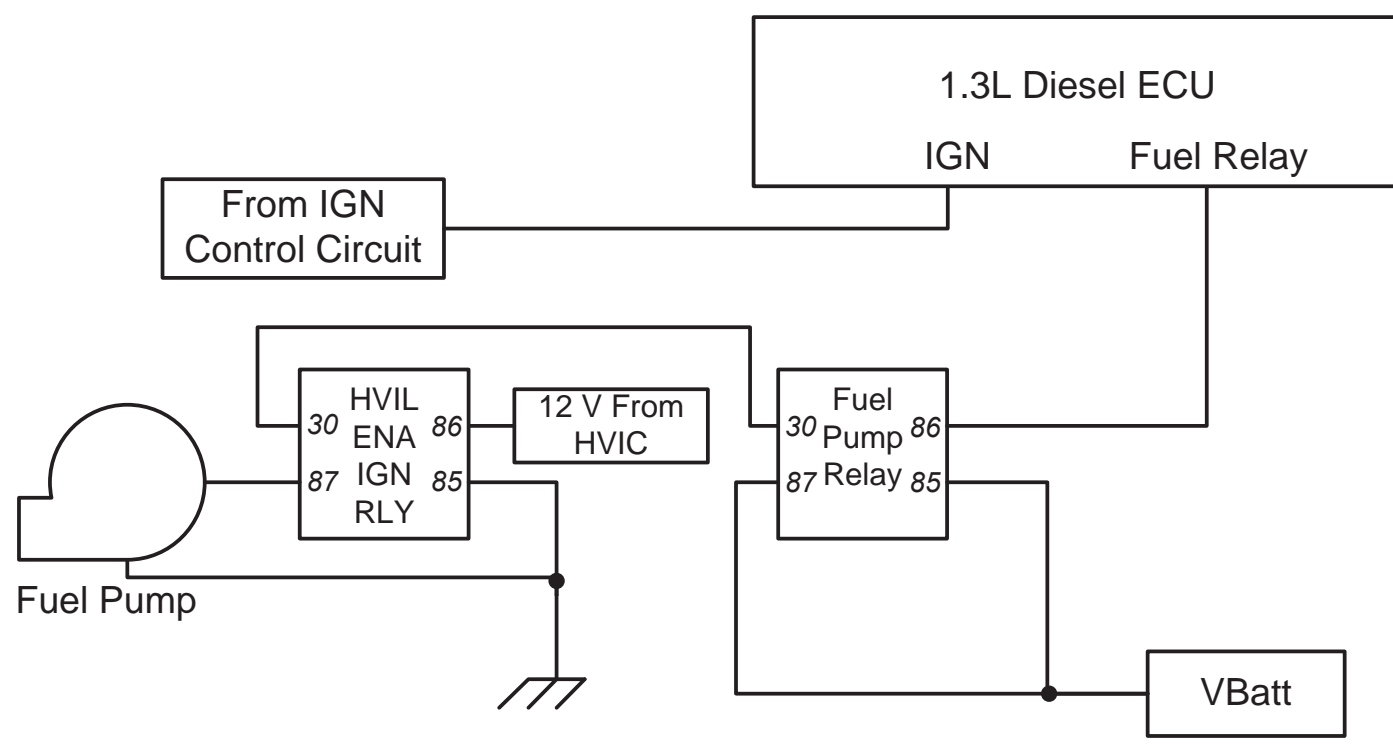

Figure 8-9: Hybrid fueling system.

\subsection{5 $\quad$ High Voltage Battery Electric System}

The high voltage battery electric system was implemented to achieve the function with extra safety features, shown in Figure 8-10. The high voltage battery consisted of four 25S2P modules, each of which had 25 battery cells in series and 2 series stacks in parallel. In the battery box, a manual disconnect switch was installed to cut the battery voltage into half during vehicle maintenance and service.

Both negative and positive high voltage battery poles were switched ON or OFF by high voltage, high current contactor relays, MAIN_N and MAIN_P, contained within the battery pack. The high voltage battery main contactors were located in the EDS. The main contactors were controlled by the BMS. The BMS followed a specific procedure to close and open the main contactors. In the close contactor procedure, the BMS checked vehicle key status, HVIL status, BMS status and contactor weld status. In the open contactor procedure, the BMS responded to the shutdown request by checking current flow and contactor weld. The contactor close request was guarded by two emergency 
disconnect switches and an inertia switch. Those three switches would override the BMS close contactor command when they were open for any hazardous reason.

Another special feature was the charger contactors to disconnect the high voltage to the TPIM when the charger was plugged in to charge the battery. The charger contactors were controlled by the vehicle run/crank status, so high voltage was only connected to TPIM during driving, not charging.



Figure 8-10: High voltage battery electrical system.

\subsubsection{High Voltage Inter-lock Loop}

HVIL was an important safety feature to protect the driver and maintainer from the possible unintended power flow from the high voltage battery system. The HVIL was a wire loop that passed through certain high voltage components, such as the high voltage battery manual disconnect switch, TPIM, BMS, emergency disconnect switch and inertia 
switch. The HVIL was used to determine if access to high voltage components was allowed. The opening of these high voltage components caused the HVIL to open. The hybrid control system reacted to the loss of HVIL continuity by opening the high voltage contactor relays and discharging the high voltage capacitors. The HVIL signal was generated by the BMS. The HVIL status was monitored by each motor control processor as well as the HCP and the BMS.

The HVIL integrated a $5 \mathrm{~V}$ circuit loop in TPIM and a $12 \mathrm{~V}$ circuit loop in high voltage battery pack, shown in Figure 8-11. The HVIL to the TPIM and to the HV battery were powered by a $5 \mathrm{~V}$ regulator circuit and a $12 \mathrm{~V}$ relay trigger board respectively. The trigger board integrated two normally open relays to control the $12 \mathrm{~V}$ power supply to the BMS, DECM ignition and fuel pump. The TPIM HVIL passed through the TPIM cover, emergency disconnect switch 1, emergency disconnect switch 2 and inertia switch, while the HV battery HVIL passed through the high voltage battery box cover and the manual disconnect switch. Any HVIL loop discontinuity would cause the TPIM to open battery main contactors and the SCU to disconnect the $12 \mathrm{~V}$ power supply to the BMS, the DECM ignition and fuel pump. Opening of the emergency disconnect switch 1, emergency disconnect switch 2 and inertia switch would disconnect the power supply to the $5 \mathrm{~V}$ regulator circuit, which caused the TPIM HVIL to be OPEN. The TPIM HVIL OPEN would cause the loss of the trigger signal (+T), which would hence open the high voltage contactors. 
High Voltage Interlock Loop (HVIL)

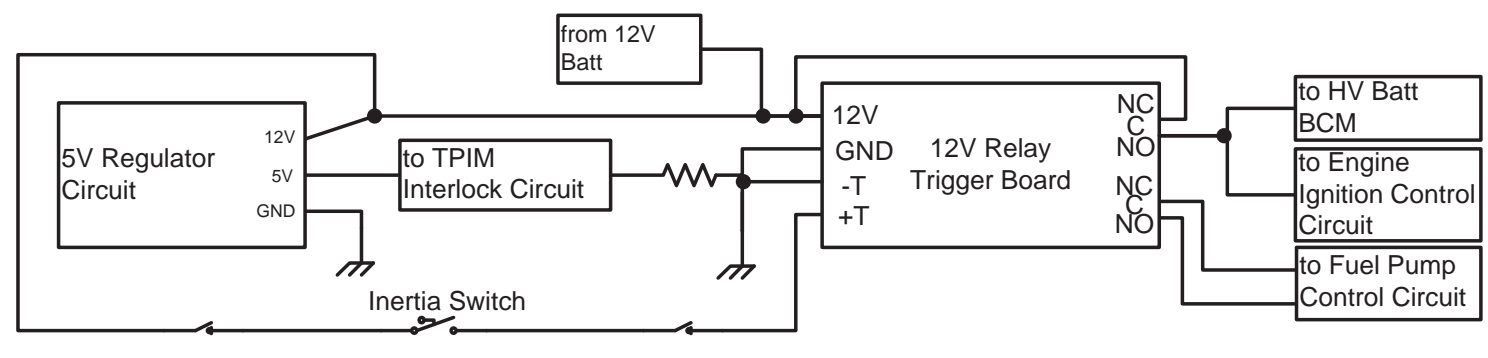

Emergency Disconnect Switch 1

Emergency Disconnect Switch 2

Figure 8-11: HVIL.

\subsubsection{High Voltage Battery Management System}

The high voltage BMS was integrated with the production control units and onboard charger to implemented designated hybrid functions, shown in Figure 8-12. The BMS can be activated exclusively with the Veh_WAKE pin or CHGA_WK pin.

Veh_WAKE signal activated the BMS in vehicle drive mode and CHGA_WK signal activated the BMS in charger mode.

The BMS was activated in vehicle drive mode by detecting the vehicle RUN/CRANK signal. The RUN/CRANK signal activated the BMS power line (PWR) and the vehicle wake signal (Veh_WAKE) as the vehicle key was turned on.

The BMS was activated in charger mode by detecting the onboard charger status. The BRUSA NLG5 charger covered the output voltages from $200 \mathrm{~V}$ DC to $520 \mathrm{~V}$ DC and power scalable from $3.3 \mathrm{~kW}$ up to $20 \mathrm{~kW}$ [79]. The charger could be programmed for different battery chemistry and different control regimes, such as CAN control or automatic operation. As the charger was plugged in to AC power, the charger controller enabled the BMS via the charger auxiliary voltage signal (CHGA_AUX). At the same 
time, the BMS was activated in charger mode via the charger wake signal (CHGA_WK). Finally, the BMS enabled the charger operation via the CHGA_ENA signal.



Figure 8-12: BMS and onboard charger integration.

\subsubsection{Maximum Discharging Power and Current}

High voltage battery maximum discharging power limit was limited by the battery temperature (BattTemp), battery state of charge (BattSOC), battery maximum discharge current (MaxDischCurrent) and battery discharge buffer (DischargeBuffer). The MaxDischCurrent was the instantaneous allowed discharge current, which would be reduced as the cell transient capability was consumed. The vehicle must stay within the MaxDischCurrent to prevent battery damage. Battery alarms would be logged by the BCM if the vehicle system exceeded this limit.

The DischargeBuffer was the transient acceptance factor indicating how soon before the BCM would start to limit its max discharging current. The vehicle controller 
used DischargeBuffer to manage the current reduction at a vehicle level, so that the battery limiting/protection didn't directly define how the vehicle managed torque during a reduction event. A 100\% DischargeBuffer indicated a large buffer to accept discharge up to MaxDischCurrent. A 0\% Discharge Buffer indicated that MaxDischCurrent was reduced to a steady-state level based on SOC and temperature, shown in Figure 9-20.

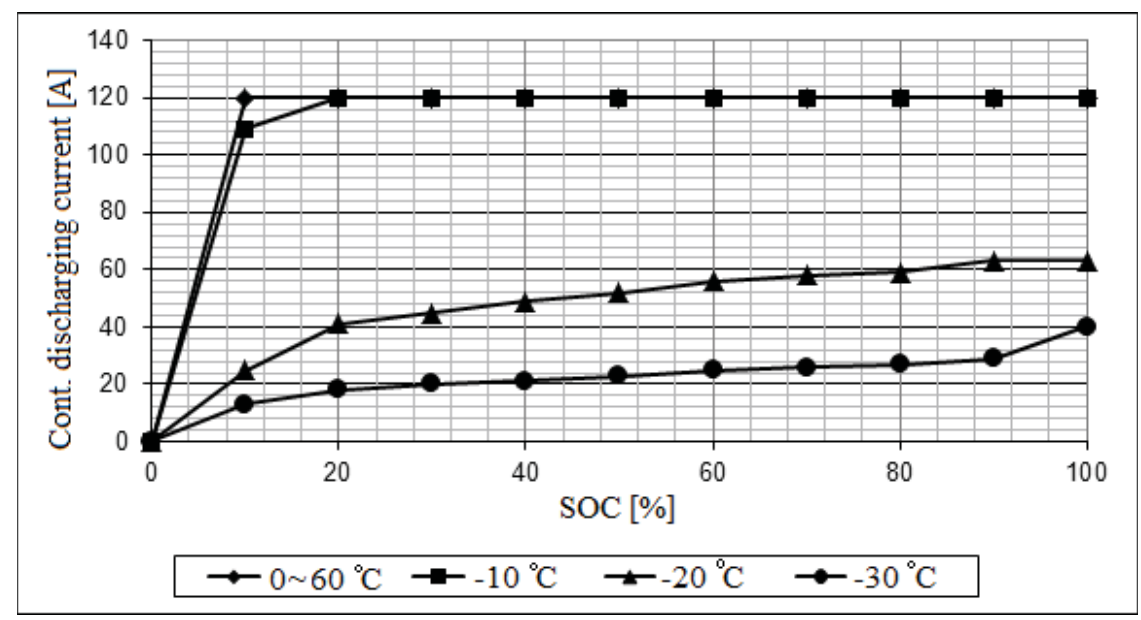

Figure 8-13: Steady-state maximum discharging current.

The high voltage battery maximum discharging power algorithm was implemented by combining the DischargeBuffer and the MaxDischCurrent, shown in Figure 8-14. The DischargeBuffer status was monitored during vehicle operation. As soon as the DischargeBuffer was set to zero, the MaxDischCurrent was constraint to the steady-state current as a function of battery temperature and SOC. 


\begin{tabular}{|c|c|}
\hline \multicolumn{2}{|c|}{ Input of DischargeBuffer, BattTemp, BattSOC, BattVolt and MaxDischCurrent } \\
\hline Yes DischargeBuffer=0 & No \\
\hline $\begin{array}{l}\text { MaxDischCurr1=fun(BattTemp, BattSoC) } \\
\text { MaxDischPwr1=Batt*MaxDischCurrent1 }\end{array}$ & MaxDischPwr2=Batt*MaxDischCurrent \\
\hline MaxDischPwr=MIN(MaxDischPwr1, MaxDischPwr2) \\
\hline
\end{tabular}

Figure 8-14: High voltage battery maximum discharging power algorithm.

\subsubsection{Maximum Charging Power and Current}

The maximum charging power \& current worked the same way as the maximum discharging power \& current described above with an instantaneous allowed charge current and charging buffer.

\subsection{Summary}

The 2-Mode HEV control system integration included the interface and communication with the $1.3 \mathrm{~L}$ diesel engine, $330 \mathrm{~V}$ high voltage battery and TPIM. The hybrid function control involved in the electronic control units including SCU, ACU, TPIM, TCM, DECM and BMS.

The detailed implementation of the hybrid powertrain control algorithm was introduced. The main hybrid function implementation included the accelerator pedal rewiring, engine ignition control, engine stop-start control, fueling system control, high voltage interlock circuit, high voltage battery control and battery power limits. 


\section{CHAPTER 9}

\section{ON-ROAD TESTS AND RESULTS}

\subsection{Introduction}

This chapter summarizes on road tests of the prototype 2-Mode HEV. The vehicle was tested on road to validate the hybrid control units, the performance and fuel economy compared to the VTS. The on road test results were discussed and compared with the analysis and simulation results to validate the designed vehicle technical specifications.

The HEV was tested at GM Milford Proving Ground and the local roads in Morgantown, WV. The GM Milford Proving Ground included the equivalent of 132 miles of roads representative of conditions found on public roadways and other specialty surfaces for vehicle testing. The vehicle was also tested on the West Virginia University (WVU) campus and in Morgantown with real world environments.

Advanced technology devices were utilized to record the vehicle performance data for further analysis. Vector CAN devices were used to record vehicle CAN signals during the test. MotoTune software was used to record extra physical I/O signals and model signals on MotoTron hybrid controllers. A Global Positioning System (GPS) tracking and recording system was used to record vehicle speed, position and road elevation information. The vehicle was tested over 500 miles over two months to validate the durability of the hybrid control system.

\subsection{Control System Calibration}

Calibration was a crucial phase in the 2-Mode HEV control system development. The calibration was the final stage of the HEV control system development to improve 
the control quality and vehicle performance. The calibration determined the attributes of the 2-Mode HEV to improve the drivability and comfort. The calibration covered the HEV control system in different operating modes.

The calibration determined HEV control parameters including:

- $\quad$ Target SOC calibration

The target SOC calibration determined a reasonable amount of energy to be balanced during the HEV operation. Too low of a target SOC put the HEV in the jeopardy of fully depleting the battery. Too high target SOC limited the amount of the energy to be used from the battery, requested more power from engine and absorbed less regenerative braking power. The battery technology used was more efficient in terms of internal resistance at the SOC around 50\% [71].

- Transmission mode shifting calibration

Transmission mode shifting affected the vehicle drivability and comfort. A bad transmission shifting calibration made the HEV stumble during the transmission shifting process. The driver didn't feel the stumble during the transmission shifting process for the good shifting calibration. The transmission shifting calibration and the engine speed control calibration combined together to improve the vehicle drivability by dynamically changing the transmission speed ratio in the EVT.

- Engine stop-start calibration

EV driving mode with engine stop improved the vehicle fuel economy. However, stopping engine too frequent depleted the battery quicker, which was later charged from either the braking power or engine power. 
- Regenerative braking power calibration

A too high regenerative braking power could stiffen the braking system and make the vehicle stumble even with small braking pedal movement. However, too small regenerative braking power command wasted the free braking energy to charge the high voltage battery and reduced the fuel economy.

- Engine power calibration

The engine provided the average power to drive the vehicle since the control strategy was charge sustaining. The engine borrowed power from the high voltage battery for assistance and returned the power to battery later on when battery SOC was low. Too little engine power sacrificed the vehicle fuel economy. Too low engine power resulted in depletion of the battery.

Based on the above discussion of the parameters effects, 10 different vehicle calibration configurations, shown in Table 9-1 were created to evaluate the vehicle fuel economy and performance. 
Table 9-1: Hybrid control system calibration configurations.

\begin{tabular}{|c|c|c|c|c|c|c|}
\hline & $\begin{array}{l}\text { Target } \\
\text { SOC }\end{array}$ & $\begin{array}{l}\text { Shift Speed } \\
\text { (Mode1 to } \\
\text { Mode 2/Mode2 } \\
\text { to Mode 1) }\end{array}$ & \begin{tabular}{|l} 
Engine \\
On/Off \\
Speed (off to \\
on/on to off) \\
\end{tabular} & $\begin{array}{l}\text { Engine_On/ } \\
\text { Off_Pwr (on } \\
\text { to off/off to } \\
\text { on) }\end{array}$ & $\begin{array}{l}\text { Regen } \\
\text { On/ } \\
\text { Off }\end{array}$ & $\begin{array}{l}\text { Eng_On } \\
\text { Always }\end{array}$ \\
\hline & (\%) & $(\mathrm{mph})$ & (mph) & (kW) & - & \\
\hline Config1 & 50 & $35 / 30$ & $20 / 15$ & $15 / 20$ & ON & NO \\
\hline Config2 & 50 & $35 / 30$ & $20 / 15$ & $15 / 20$ & OFF & NO \\
\hline Config3 & 50 & $35 / 30$ & - & - & ON & YES \\
\hline Config4 & 50 & $35 / 30$ & - & - & OFF & YES \\
\hline Config5 & 50 & $35 / 30$ & $20 / 15$ & $10 / 15$ & ON & NO \\
\hline Config6 & 50 & $35 / 30$ & $20 / 15$ & $15 / 20$ & ON & NO \\
\hline Config7 & 45 & $35 / 30$ & $20 / 15$ & $15 / 20$ & ON & NO \\
\hline Config8 & 50 & $35 / 30$ & $23 / 20$ & $15 / 20$ & ON & NO \\
\hline Config9 & 50 & $40 / 35$ & $27 / 22$ & $15 / 20$ & ON & NO \\
\hline Config10 & 50 & $40 / 35$ & $20 / 15$ & $15 / 20$ & ON & NO \\
\hline
\end{tabular}

\subsection{Test Cycles and Test Results}

\subsubsection{On Road Safety Evaluation Test}

The purpose of the On Road Safety Evaluation (ORSE) test, shown in Figure 9-1, was to evaluate the safe functionality of the prototype 2-Mode HEV vehicle prior to on road tests. The on road safety test ensured that the vehicle was safe to operate at city and highway speeds. The ORSE tests helped to ensure a high level of vehicle safety before further on road tests. 


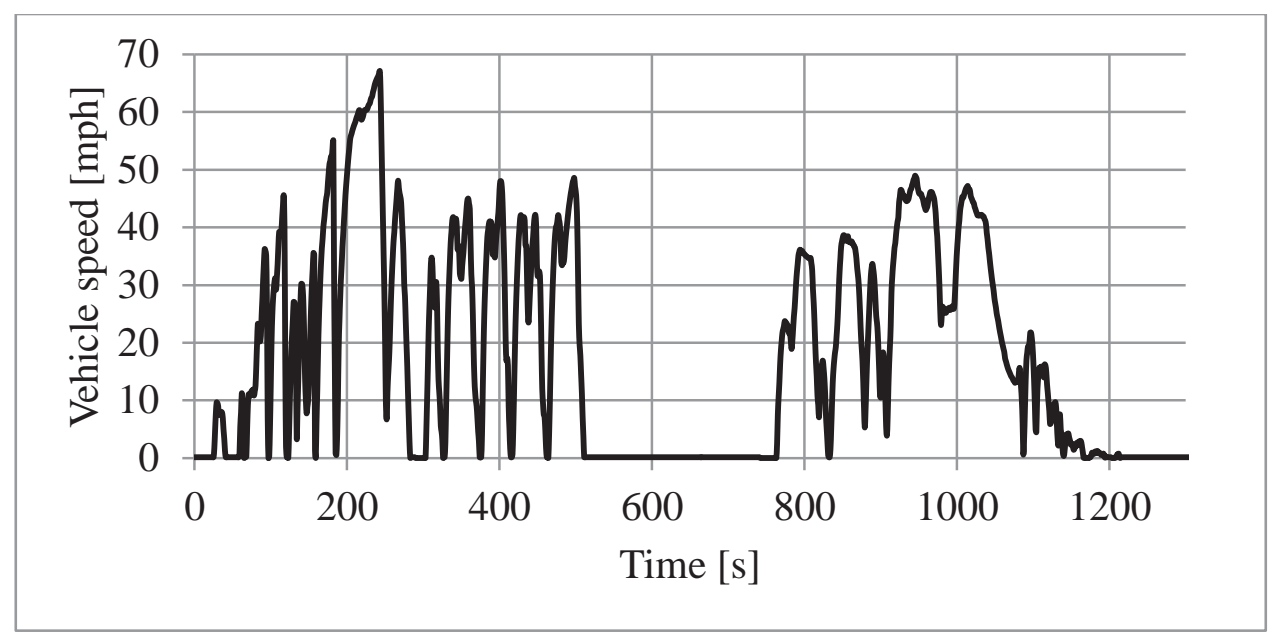

Figure 9-1: On road safety test.

The temperature shown in the Figure 9-2 indicated that the temperature of the engine, $\mathrm{HV}$ battery pack and transmission temperature were stable in a safe range. The temperatures of the engine, HV battery and transmission increased in the beginning and were stabilized at the end when the cooling system started to work.

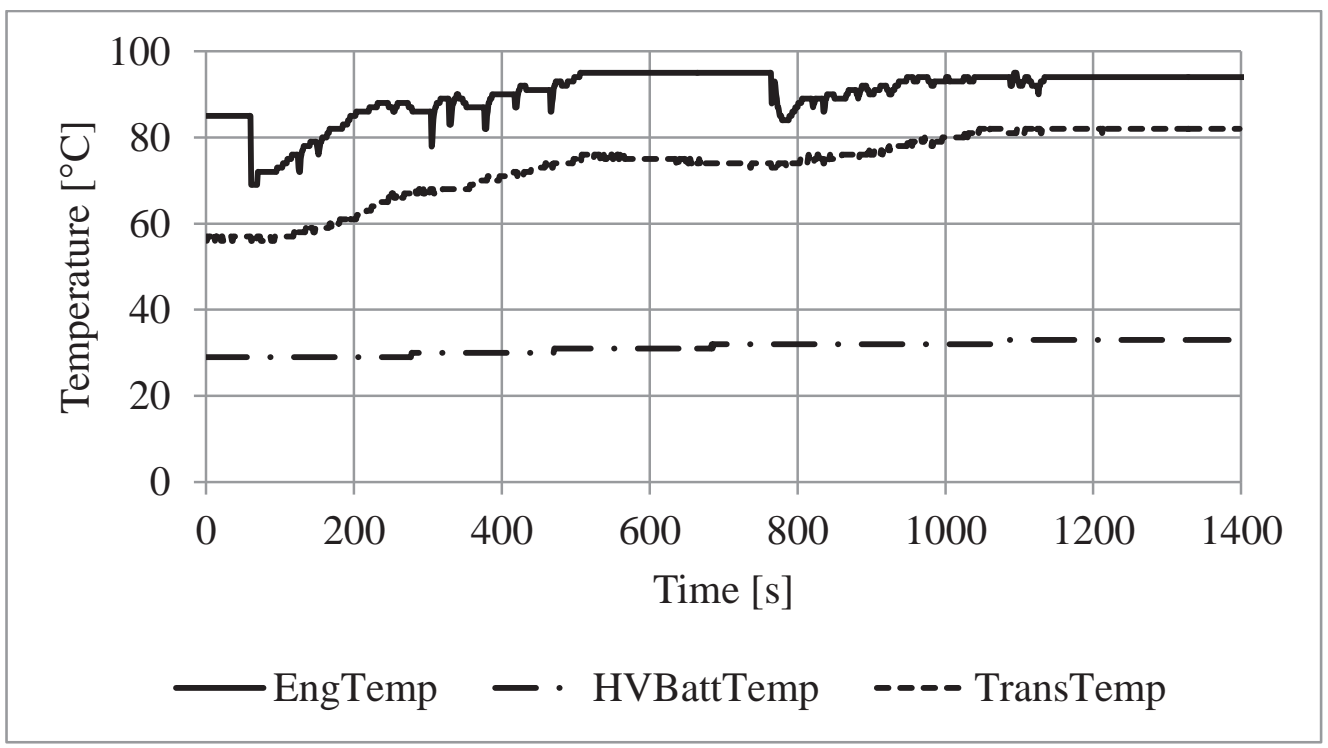

Figure 9-2: ORSE test components temperature diagram.

The engine operating diagram shown in the Figure 9-3 and Figure 9-4 indicated the engine's operation during the ORSE test. The engine diagram validated that the 
engine speed and torque output worked in the nominal operating range. From Figure 9-4, the engine operated 56\% time in the efficiency area above 35\% and 64\% time in the efficiency area above 30\%.

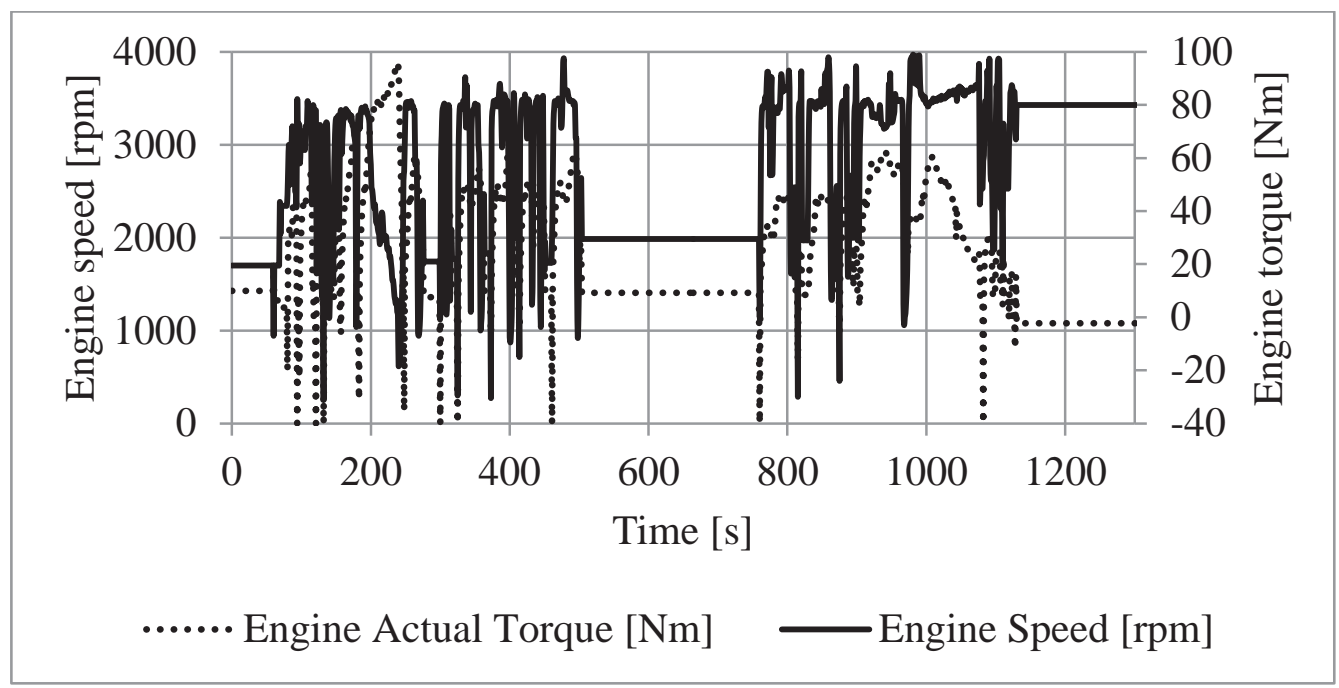

Figure 9-3: ORSE test engine operating vs. time.

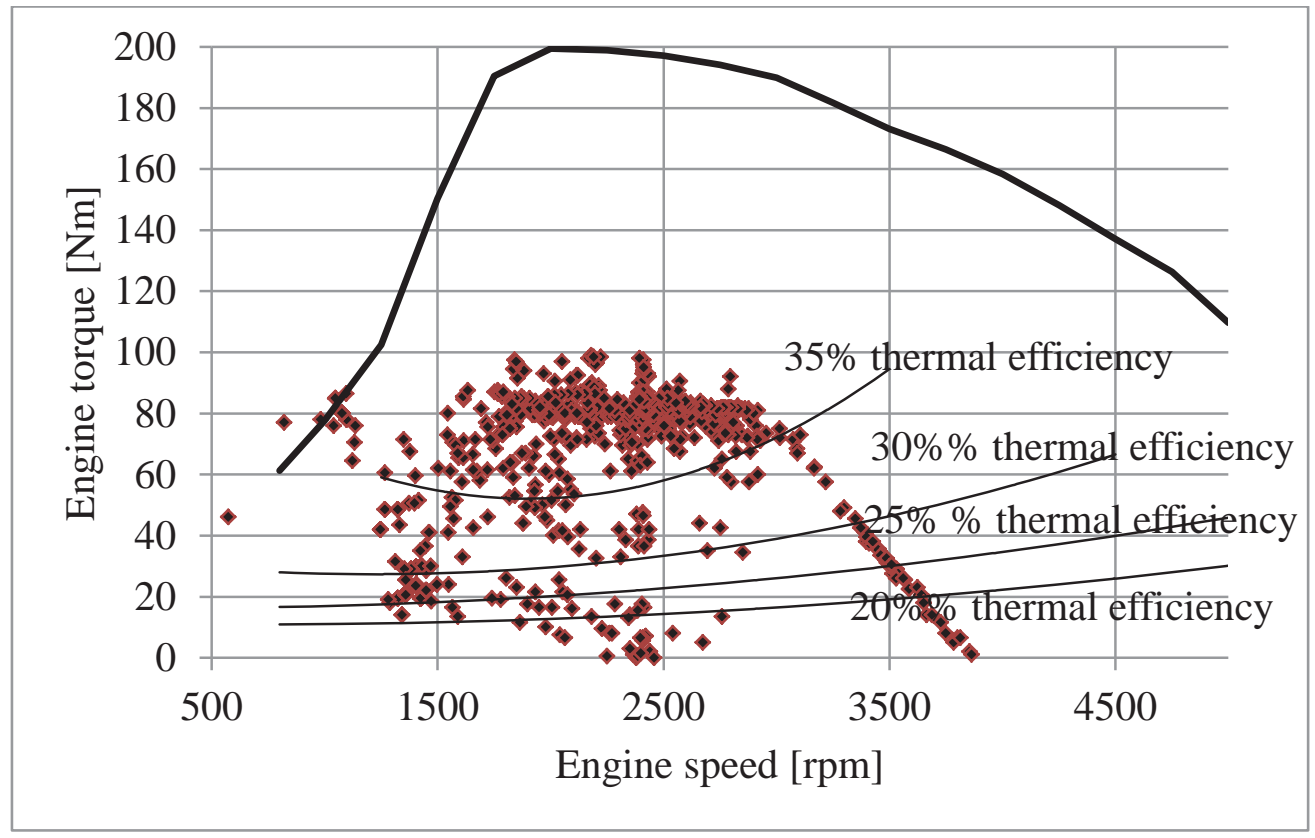

Figure 9-4: ORSE test engine operating vs. engine efficiency. 
The HV battery diagram in the Figure 9-5 validated the battery's operation during the ORSE test. The battery temperature was within $33^{\circ} \mathrm{C}$. The maximum, minimum and average discharging current were $156 \mathrm{~A}, 83 \mathrm{~A}$ and $20 \mathrm{~A}$, which were in the nominal operating ranges. The battery module voltage floated around the nominal voltage, $83 \mathrm{~V}$.

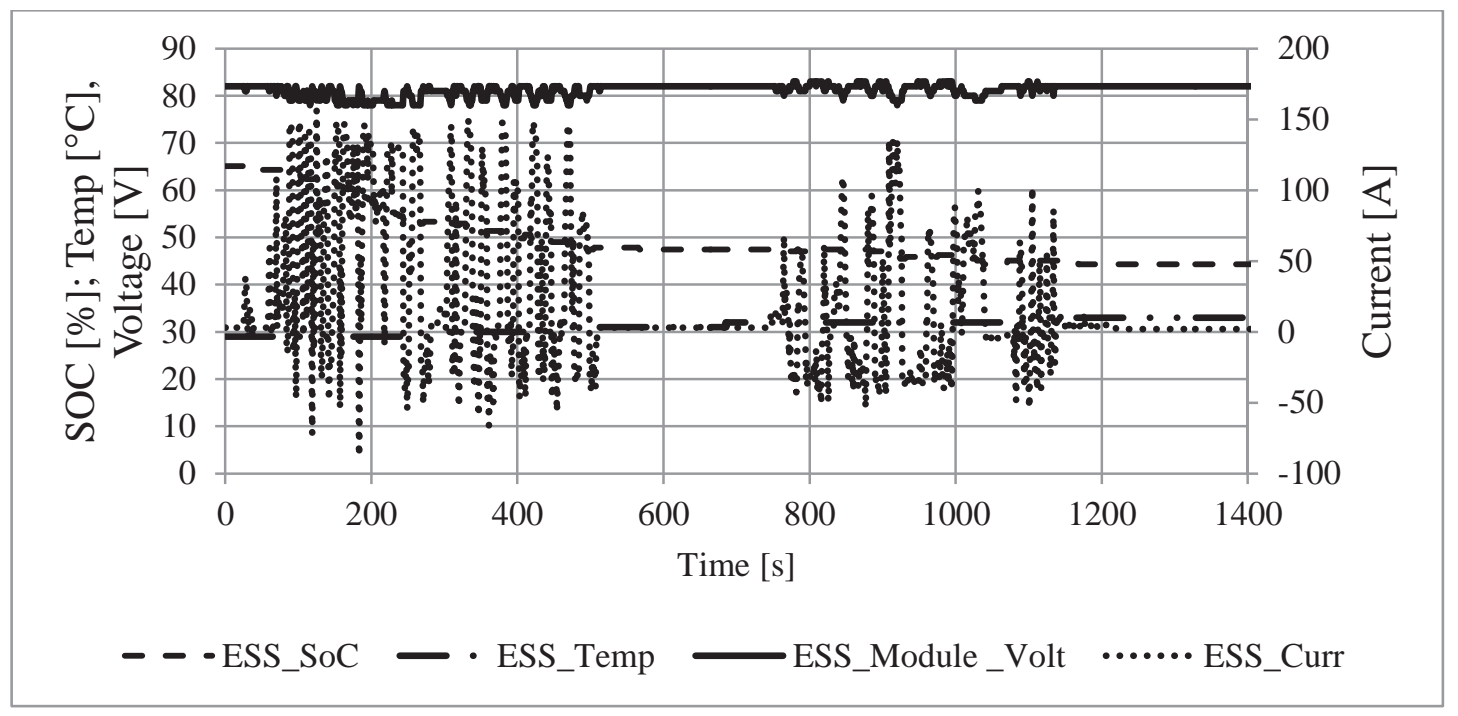

Figure 9-5: ORSE test high voltage battery operation.

\subsection{2 $\quad$ Fuel Economy (FE) Test}

On-road fuel consumption testing required the development of test cycles to be used to compare the fuel economy and vehicle overall performance for different control calibrations. First, locations around West Virginia University with minimal elevation change were found to minimize grade-related power as much as possible. A three mile stretch of US Route 19 was selected for highway testing and the outer loop around the Morgantown Mall was chosen for conducting city driving testing. Once the locations were selected, city and highway driving cycles were developed. Figure 9-6 is a plot of the developed Morgantown Urban Driving Schedule (MUDS) and Figure 9-7 is the R19HW cycle. These plots show vehicle speed vs. time. The geometry positions of the 
roads are shown in Figure 9-8 and Figure 9-9. The MUDS trace was modeled after the EPA's UDDS cycle with multiple accelerations and decelerations and speeds never exceeding $45 \mathrm{mph}$. This schedule was designed to test the effects of modifying the Engine STOP-START control, changing transmission shift points and regenerative braking on the vehicle's fuel economy and performance. The total cycle distance was over 3 miles. The R19HW trace consisted of a moderate acceleration to $55 \mathrm{mph}$ followed by a reduction of speed to $45 \mathrm{mph}$ after about one mile. This cycle was designed to test the vehicle over extended periods of Mode 2 engine ON operation at engine speeds above 2,500 rpm. The total R19HW cycle distance was also just over 3 miles. These cycles were also imported and simulated in PSAT to validate the vehicle model.

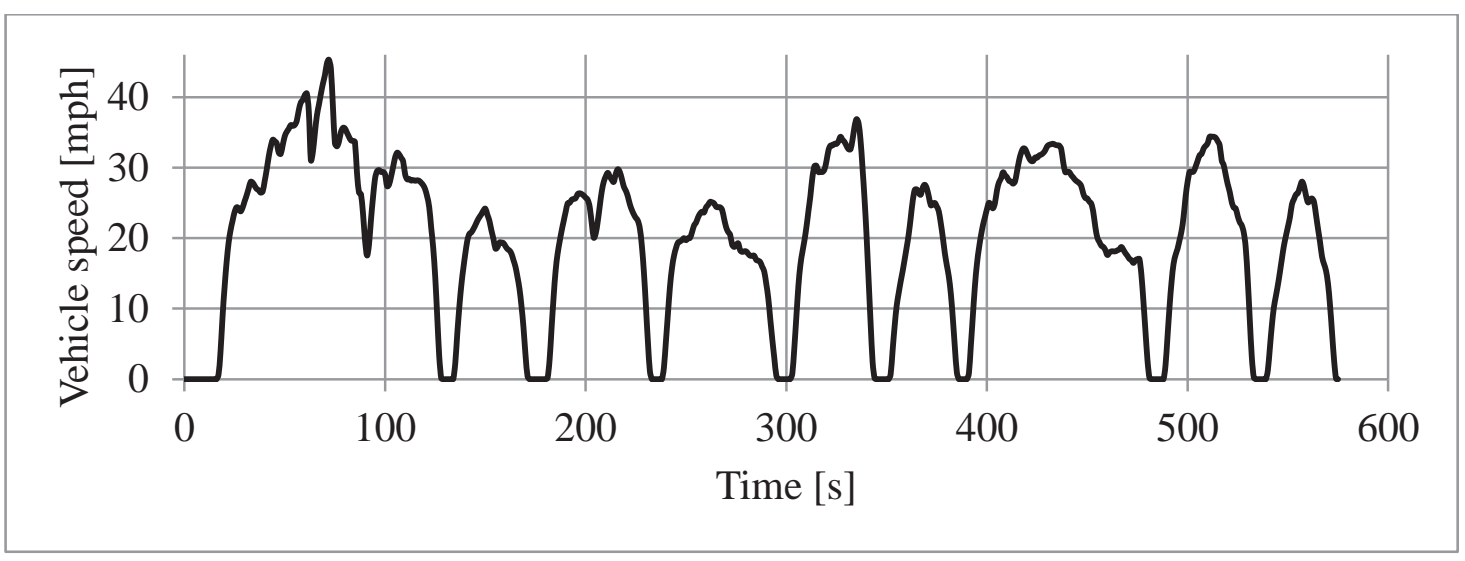

Figure 9-6: Morgantown urban drive schedule (MUDS). 


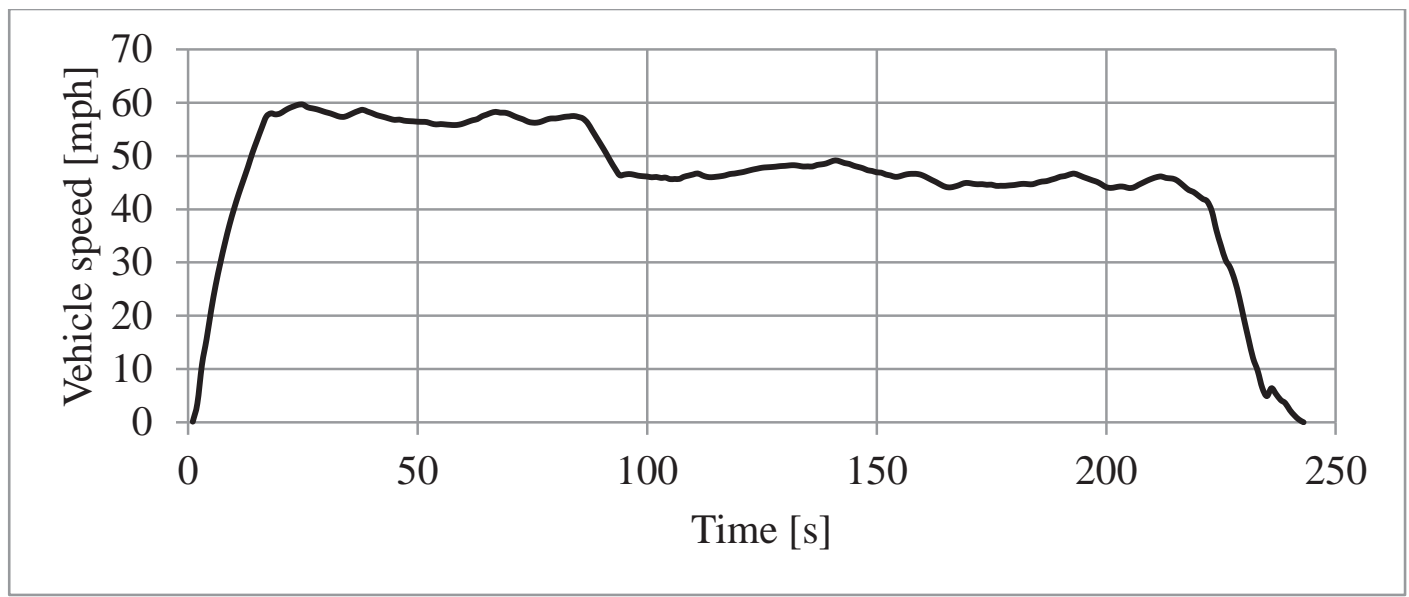

Figure 9-7: Route 19 highway (R19HW).

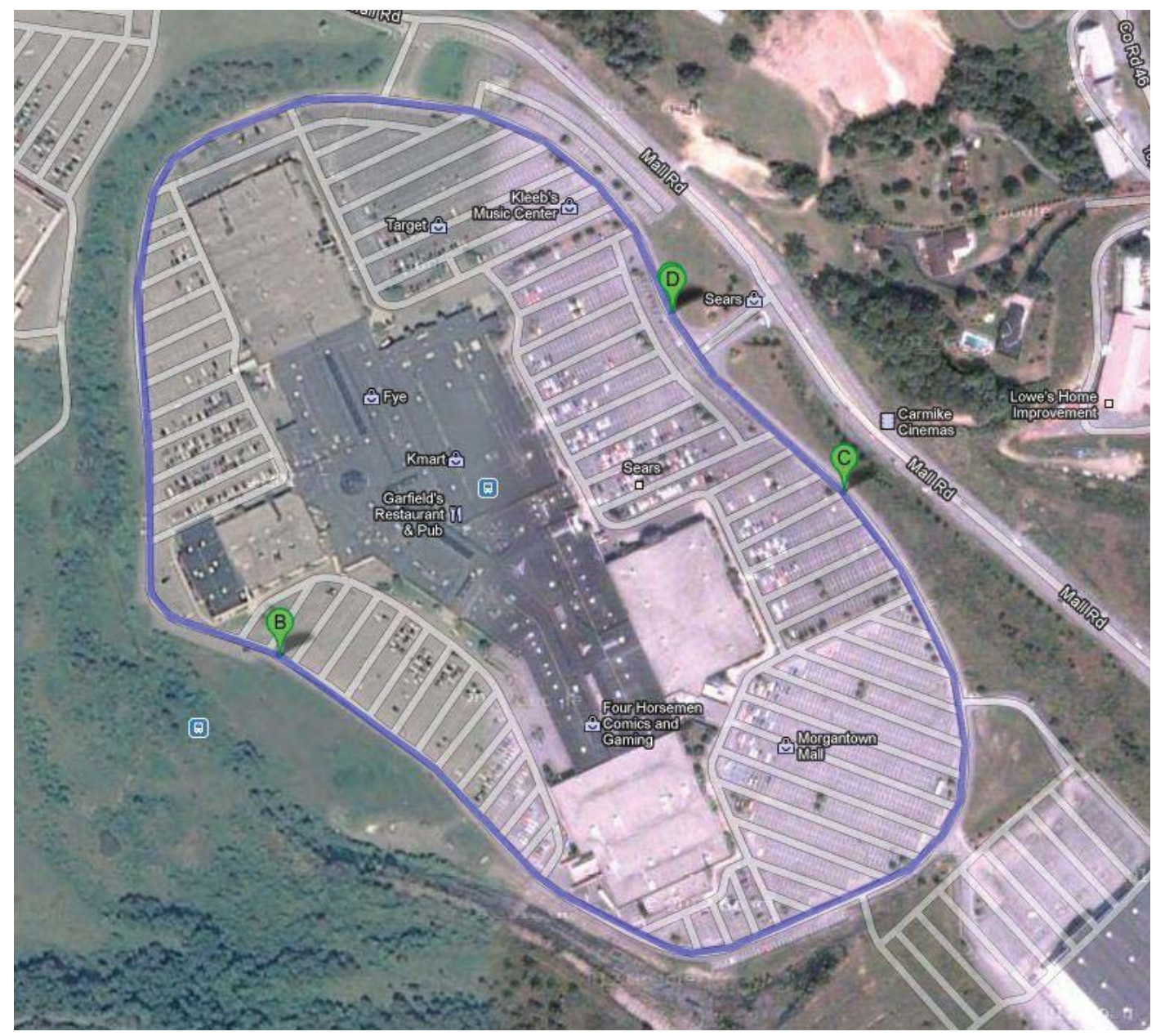

Figure 9-8: MUDS road Google map. 




Figure 9-9: R19HW road Google map.

The road grade was calculated using an absolute air pressure sensor and converting the pressure reading to an elevation, as shown in Eq. 9-1. This elevation data was integrated with respect to vehicle speed to get instantaneous grade data. This grade data was used in the PSAT cycle to more accurately simulate the real test cycle.

$$
\mathrm{h}=\frac{\mathrm{T}}{\mathrm{L}}\left[\left(1-\frac{\mathrm{P}}{101.325 \mathrm{kPa}}\right)^{\frac{\mathrm{RL}}{g M}}\right]
$$

Where: $\mathrm{h}$ is the elevation in meters; $\mathrm{T}$ is the measured temperature in $\mathrm{K}$; $\mathrm{L}$ is temperature lapse rate constant, $0.0065 \mathrm{~K} / \mathrm{m}$; $\mathrm{P}$ is the measured barometric pressure in $\mathrm{kPa}$; R is universal gas constant, $8.3145 \mathrm{~J} /(\mathrm{mol} \cdot \mathrm{K})$; $\mathrm{g}$ is the earth surface gravitational acceleration, $9.8067 \mathrm{~m} / \mathrm{s}^{2} ; \mathrm{M}$ is the molar mass of dry air, $0.0290 \mathrm{~kg} / \mathrm{mol}$. 
Fuel consumption was calculated based on the amount of fuel used and the distance traveled, with provisions for PHEV fuel consumption based on the J1711 SAE standard.

Multiple signals were recorded during vehicle testing to calculate fuel economy. These signals included the instantaneous fuel flow rate into the engine (g/s), the vehicle speed (kph), the high voltage battery state of charge (\%), and engine stop-start status. All of this information was recorded directly from the vehicle's CAN bus using VECTOR's CANoe ${ }^{\circledR}$ software.

The ten calibration configurations were implemented in the hybrid control system for the MUDS and R19HW tests. The best overall configuration in terms of combined fuel economy and vehicle performance was achieved in configuration 9 with Up/Down shifted at 40/35 mph, engine stop-start at $27 / 22 \mathrm{mph}$, and a target state of charge of 50\%. Regenerative braking improved fuel economy for every control algorithm.

The on-road tests results were used to validate the prototype HEV design targets on the vehicle performance and the fuel efficiency in the simulation. Using vehicle speed and elevation data collected while driving the MUDS and R19HW test routes, simulated routes were developed for PSAT. A model was developed that correlated closely with the actual vehicle including the same powertrain components and hybrid control strategy. This model was simulated across both the MUDS and R19HW test cycles and the results were compared to the actual measured fuel economy. The vehicle on-road tests with the local city and highway cycle reached 24.5/31.5 MPG, which achieved a 23.8\% combined fuel economy improvement compared with the mule vehicle, 19.0/26.0 MPG, shown in 
Table 9-2. Table 9-3 lists the simulation and actual fuel economy results for the MUDS city cycle, R19HW highway cycle, and the overall combined fuel economy.

Table 9-2: Fuel economy comparison of the 2-Mode HEV to the mule vehicle.

\begin{tabular}{|l|c|c|c|}
\hline & Mule vehicle (MPGge) & Actual (MPGge) & Difference \\
\hline MUDS & 19.0 & 24.5 & $29.0 \%$ \\
\hline R19HW & 26.0 & 31.5 & $21.2 \%$ \\
\hline Combined & 22.9 & 28.4 & $24.0 \%$ \\
\hline
\end{tabular}

The fuel economy of 2-Mode HEV in the on-road test was $2.1 \%$ less than that in the PSAT model. The difference could be caused by differences in the control program between in the TPIM and in the PSAT model. The TPIM algorithm to control the electric motors and the inverter were unknown to the WVU controls team. The difference may affect the electric motor performance, hence the efficiency of electric motors and the power electronics.

Table 9-3: Fuel economy comparison of on-road test results to the PSAT model.

\begin{tabular}{|l|c|c|c|}
\hline & PSAT (MPGge) & Actual (MPGge) & Difference \\
\hline MUDS & 28.4 & 24.5 & $-13.7 \%$ \\
\hline R19HW & 29.4 & 31.5 & $7.1 \%$ \\
\hline Combined & 28.8 & 28.4 & $-2.1 \%$ \\
\hline
\end{tabular}

The engine control algorithm, the transmission shifting algorithm and the high voltage battery SOC sustaining controller were validated according to the on-road test results. In the hybrid control program, the engine was turned on and off based on the vehicle speed, the engine power command and the accelerator pedal percentage. The 
transmission was upshifted from Mode 1 to Mode 2 at $40 \mathrm{mph}$ and downshifted to Mode 1 at $35 \mathrm{mph}$. The target SOC of the HV battery was 50\%. The test results for this control algorithm and powertrain components performance are discussed below.

\subsubsection{Engine Performance}

The engine efficiency was improved over the MUDS cycle by turning the engine off and operating the engine within the high efficiency area. The engine was turned on only $41 \%$ of the time during the MUDS cycle, shown in Figure 9-10. When the engine was turned on, the engine operating points were located in the high efficiency area by limiting the engine power output to a high level, shown in Figure 9-11.

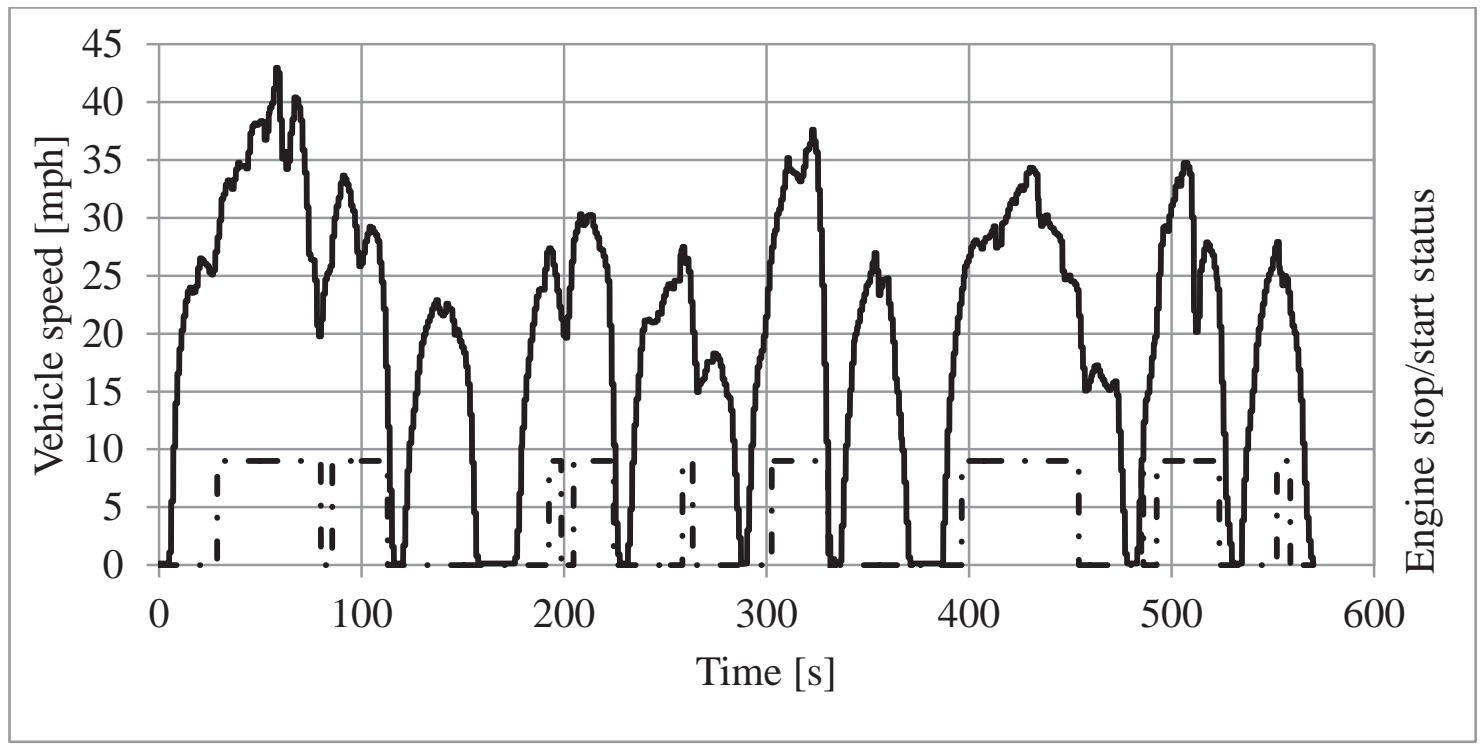

Figure 9-10: Engine stop-start status over the MUDS cycle. 


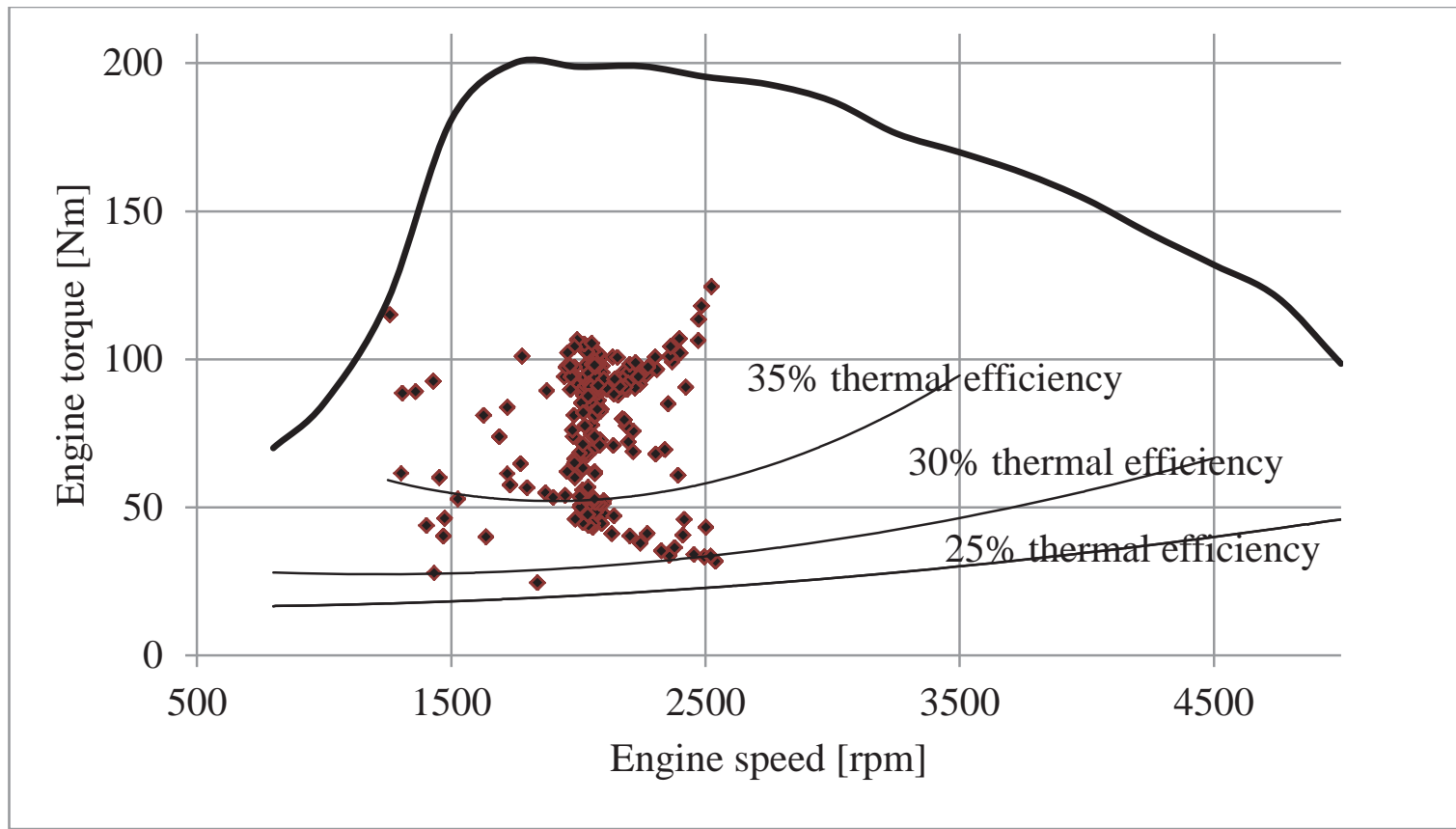

Figure 9-11: Engine operating points over the MUDS cycle.

The engine speeds on the R19HW cycle were relatively concentrated around 270 $\mathrm{rad} / \mathrm{s}(2,578 \mathrm{rpm})$ compared with those on the MUDS cycle because the engine speed was commanded based on the vehicle speed, which did not change over a wide range on the R19HW cycle, shown in Figure 9-12. The engine torque output was changed based on the vehicle road load condition and the high voltage battery SOC status. The engine efficiency was improved by commanding the engine to work at high power output. 


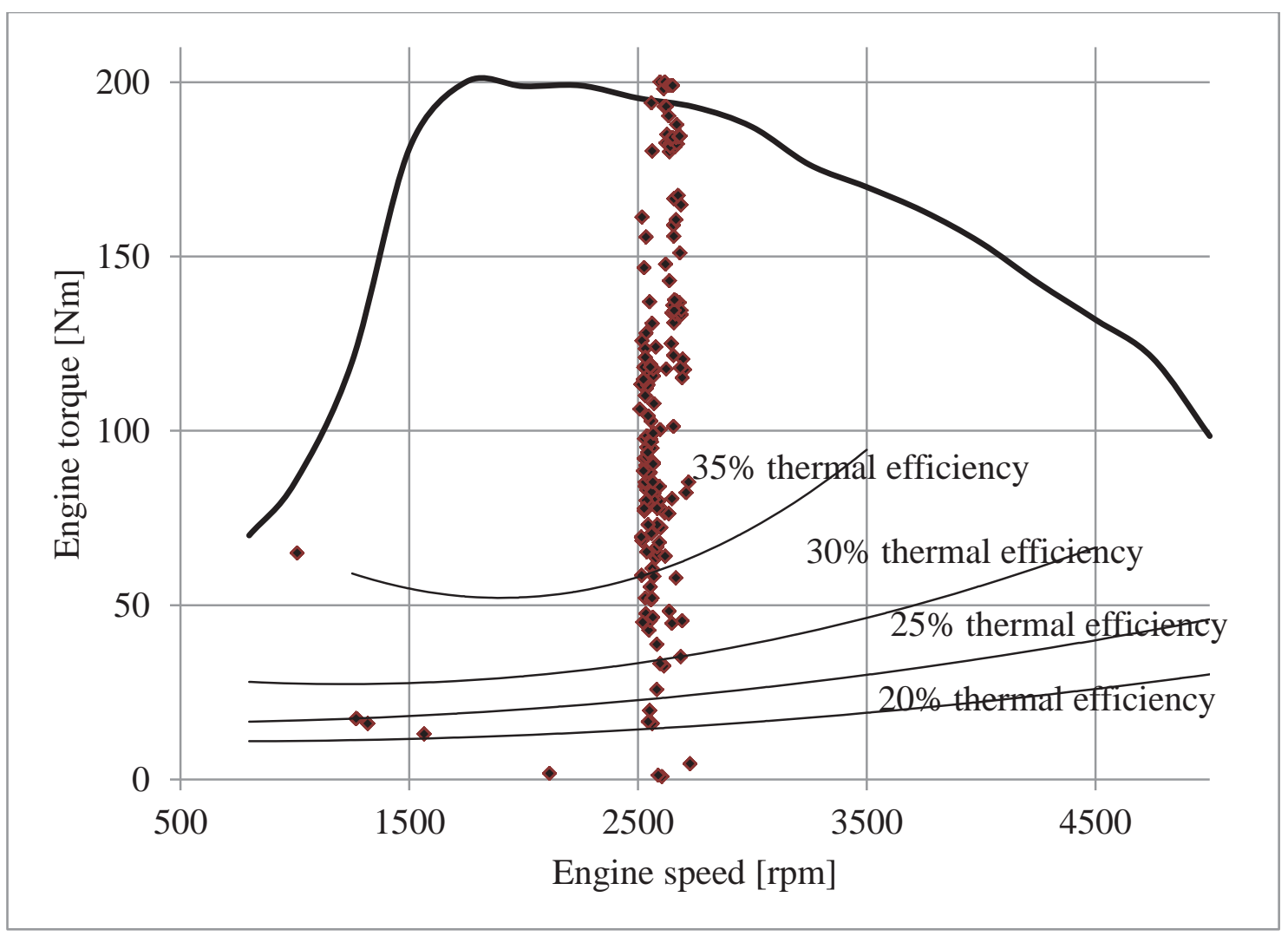

Figure 9-12: Engine working points on the R19HW cycle.

\subsubsection{2 $\quad$ Transmission Shifting}

In the transmission shifting control algorithm, the transmission was shifted into Mode 2 when the vehicle speed was above $40 \mathrm{mph}$, and was shifted back to Mode 1 at 35 mph. When the engine is shut down, the transmission can only be operated in Mode 1. The intermediate transmission modes were inserted between EVT modes for shifting protection purposes. The transmission shifting algorithm was validated with the MUDS and the R19HW on-road test results, shown in Figure 9-13 and Figure 9-14. In the MUDS cycle, the transmission shifting between Mode 1 and Mode 2 followed the control algorithm. In the R19HW cycle, the transmission was operating in Mode 2 most time because the vehicle was at a high speed, except at the end of the test cycle when the vehicle decelerated to a stop. 




Figure 9-13: Transmission shifting in MUDS cycle.

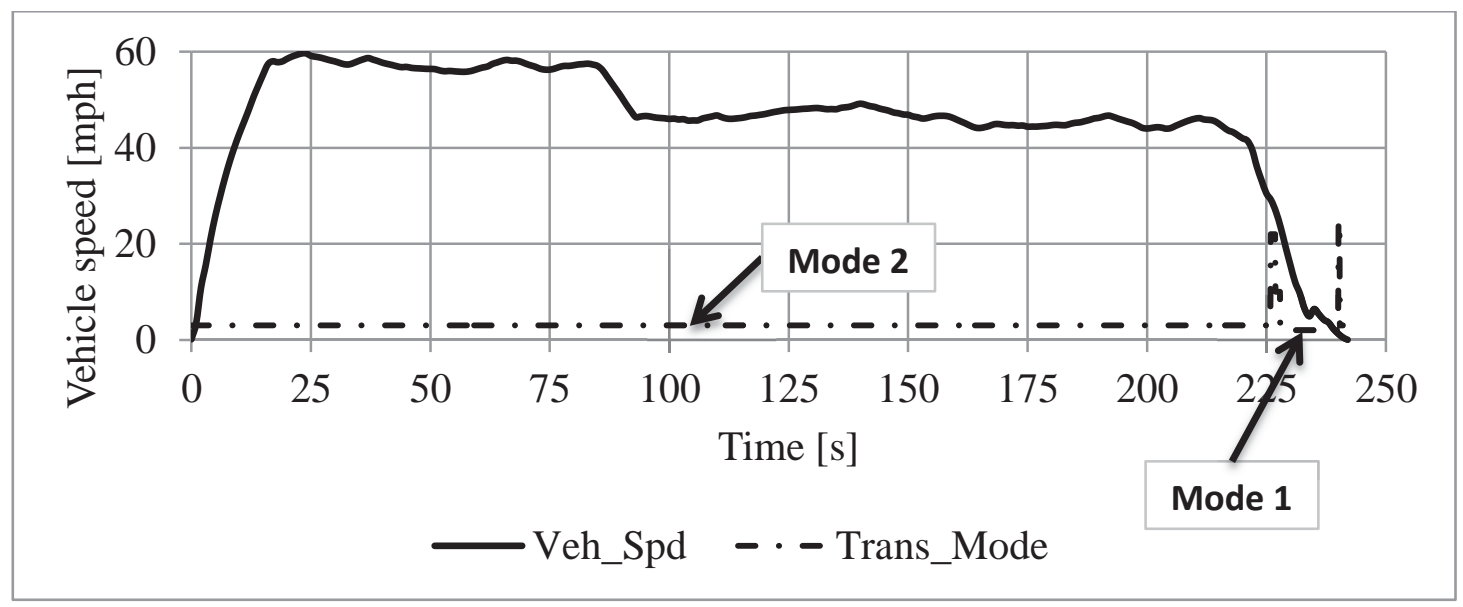

Figure 9-14: Transmission shifting in R19HW cycle.

\subsubsection{3 $\quad$ High Voltage Battery SOC Sustaining Algorithm}

A high voltage battery SOC sustaining control algorithm was implemented in the hybrid control program to keep the target SOC near 50\%. During the MUDS cycle, the high voltage battery SOC was sustained around 46\%, shown in Figure 9-15. The high voltage battery can be charged with the engine power and the regenerative braking power. The positive current shows the power discharging from the battery and the negative current shows the power charging to the battery. The Figure 9-16 shows the high voltage SOC status during the R19HW test cycle. The SOC was sustained around 
the target SOC using the PI controller. It was found that the charging power during the R19HW cycle was not as high as that in the MUDS cycle, because there was not much braking pedal depression during the R19HW cycle.

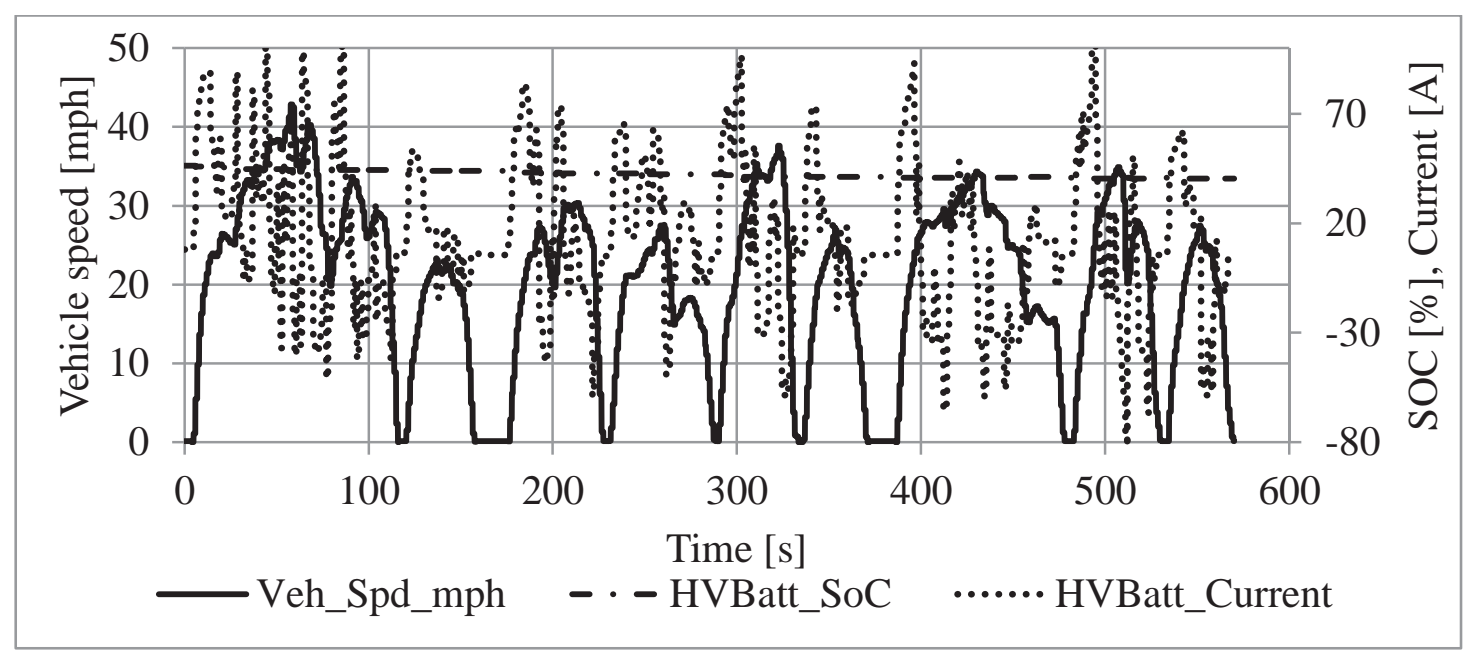

Figure 9-15: High voltage battery SOC sustaining in MUDS cycle.

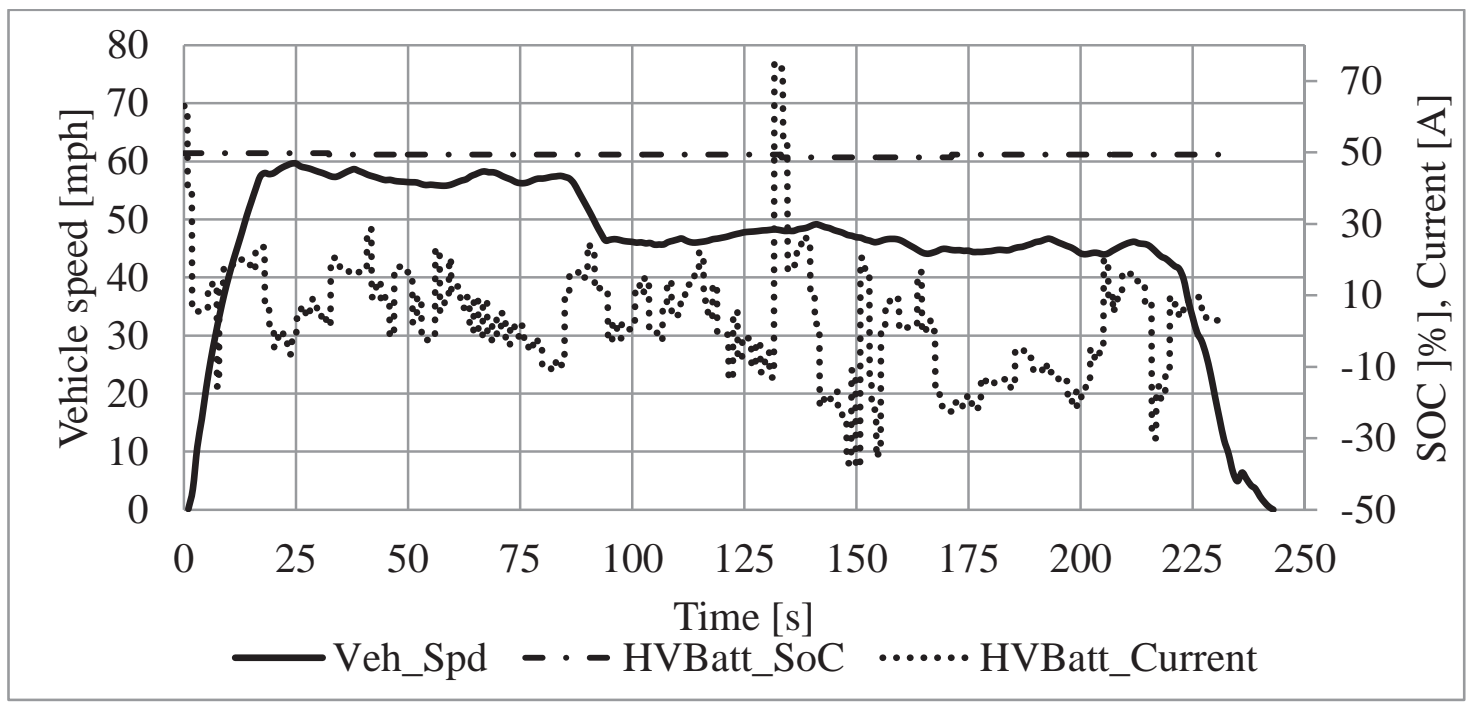

Figure 9-16: High voltage battery SOC sustaining in R19HW cycle.

\subsubsection{4 $\quad$ Motor Performance}

During MUDS test, the M/G1 operated mostly in a speed range of $-4,000 \mathrm{rpm}$ to $1,000 \mathrm{rpm}$ and in a torque range of $-100 \mathrm{Nm}$ to $150 \mathrm{Nm}$. The M/G1 worked in all 4 
quadrants, shown in Figure 9-17. The $1^{\text {st }}$ and $3^{\text {rd }}$ quadrants were the motoring mode. The $2^{\text {nd }}$ and $4^{\text {th }}$ quadrants were the generating mode. The operating points closer to the speed axis and torque axis had lower efficiency. From the test record data, the M/G1 worked in the motoring mode for $45 \%$ of the cycle and in the generating mode for $55 \%$ of the cycle respectively. The average positive speed and negative speed were $90 \mathrm{rpm}$ and -2,600 rpm. The average positive torque and negative torque were $30 \mathrm{Nm}$ and $18 \mathrm{Nm}$ not counting the points when the torque values were zero. The M/G1 worked at points with high speed but no torque when the vehicle was in EV mode and the vehicle was mainly driven by M/G2.

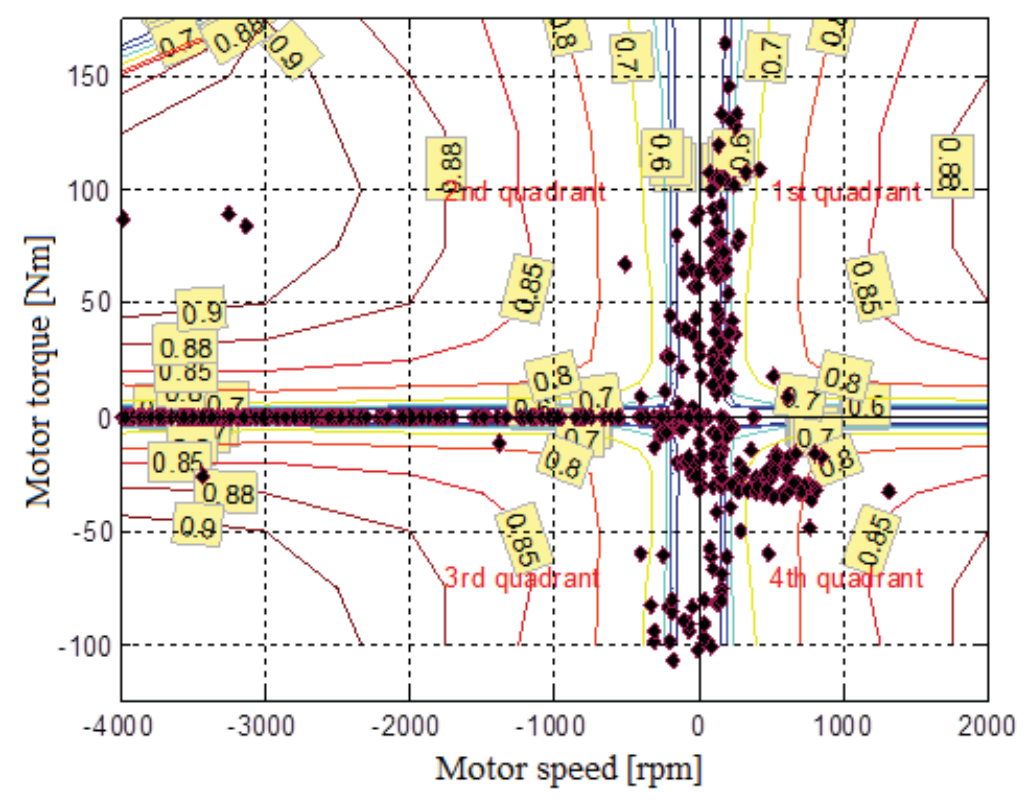

Figure 9-17: M/G1 operation in MUDS cycle.

During the MUDS test, the M/G2 operated in a single direction of the speed ranged between $0 \mathrm{rpm}$ to 4,000 rpm. The M/G1 torque worked mainly in the range of -80 $\mathrm{Nm}$ to $60 \mathrm{Nm}$, operating mostly in the $1^{\text {st }}$ quadrant during motoring and in the $4^{\text {th }}$ quadrant during generating, as shown in Figure 9-18. The M/G2 speed was in the positive direction because the M/G2 followed the vehicle driving direction. The M/G2 
speed was relatively higher compared with the M/G1 speed. The motor efficiency

increased as the motor torque command increased. The maximum motoring power was $22 \mathrm{~kW}$ and the maximum generating power reached $33 \mathrm{~kW}$.



Figure 9-18: M/G2 operation in MUDS cycle.

During the R19HW cycle, the M/G1 operated only in the $1^{\text {st }}$ quadrant, as shown in

Figure 9-19. The M/G1 worked only in motoring mode because of the high torque commands of the vehicle. No power from M/G1was converted to charge the battery. The average M/G1 speed and torque were $800 \mathrm{rpm}$ and $90 \mathrm{Nm}$, which were much higher compared with those in the MUDS cycle because the R19HW demanded more power and had higher speed. The M/G2 speeds were located mainly in two areas, one in the area around $700 \mathrm{rpm}$ and the other in the area around 1,400 rpm. This was because the R19HW speed profile included the speeds mainly at $60 \mathrm{mph}$ and $45 \mathrm{mph}$. The M/G1 efficiency was improved to a much higher level as a result of the increase of the speed and torque. The M/G1 operating efficiency around the 700 rpm area was between 0.70- 
0.80 , and the efficiency around $1,400 \mathrm{rpm}$ was between $0.80-0.85$. The motor torque reached as high as145 Nm during motoring. The M/G1 maximum motoring power reached as high as $7.3 \mathrm{~kW}$.

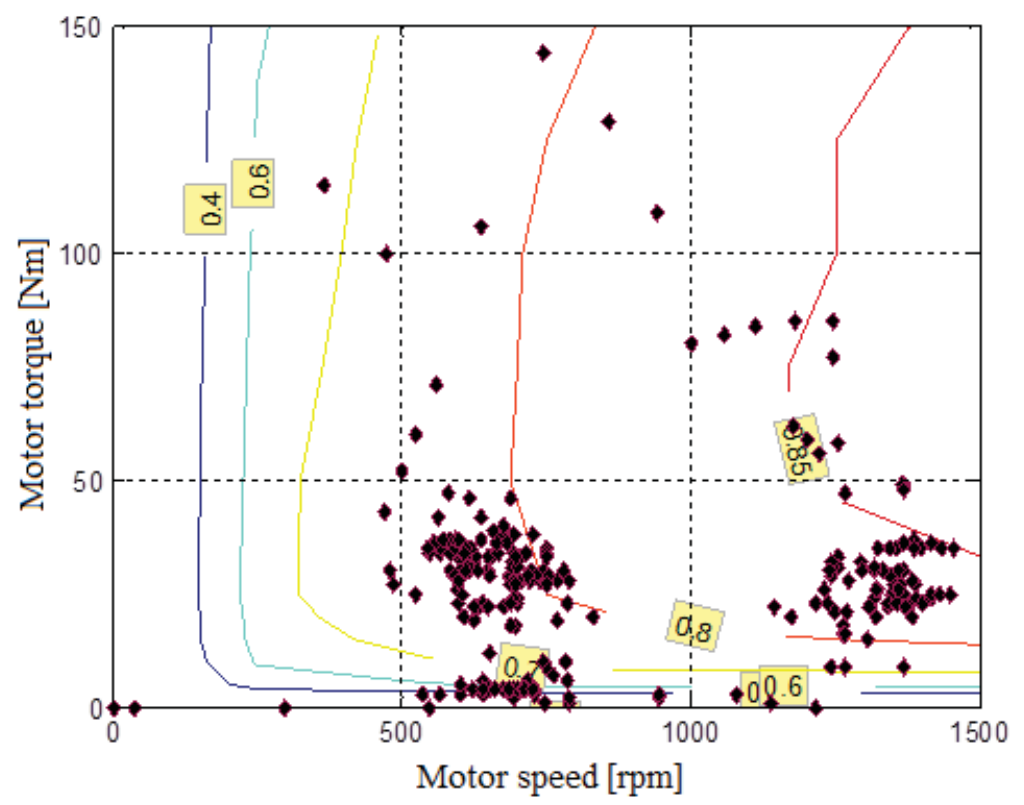

Figure 9-19: M/G1 operation on R19HW cycle.

The M/G2 operated mostly in a speed range between 3,500 rpm and 4,000 rpm and in a torque range of $-40 \mathrm{Nm}$ to $30 \mathrm{Nm}$, as shown in Figure 9-20. The M/G2 operated in a single positive direction. The M/G2 speed was focused in two clusters around 3,600 rpm and 4,000 rpm. This was also true for M/G1. The average speed of the M/G2 was 3,800 rpm. The average motoring torque and generating torque were $9 \mathrm{Nm}$ and $-17 \mathrm{Nm}$. The maximum motoring power and maximum generating power were $10.7 \mathrm{~kW}$ and 15 $\mathrm{kW}$. 


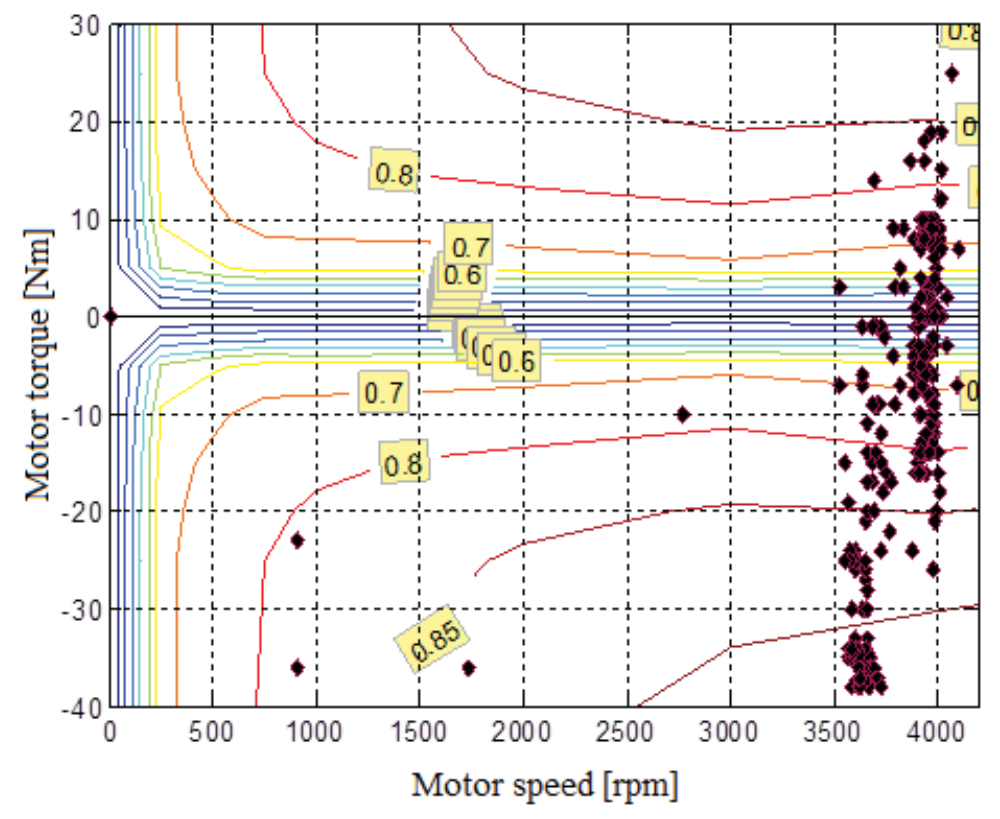

Figure 9-20: M/G2 operation on R19HW cycle.

\subsection{3 $\quad$ Autocross Test}

The autocross test demonstrated the 2-Mode HEV's ability to navigate a serpentine cone-lined course and tested the vehicle's handling capabilities. The vehicle was driven by a professional test driver to finish the handling course in the shortest time possible. The 2-Mode HEV finished the autocross test in $61 \mathrm{~s}$, which was $3 \mathrm{~s}$ longer than the stock vehicle.

\subsection{4 $\quad$ Acceleration Test}

The Acceleration and Braking tests evaluated the vehicle's ability to accelerate and decelerate. The vehicle was tested to demonstrate the best acceleration possible given the vehicle architecture design from 0-60 mph and 50-70 mph, shown in Figure 9-21, as well as stopping distance from a speed of $60 \mathrm{mph}$. 


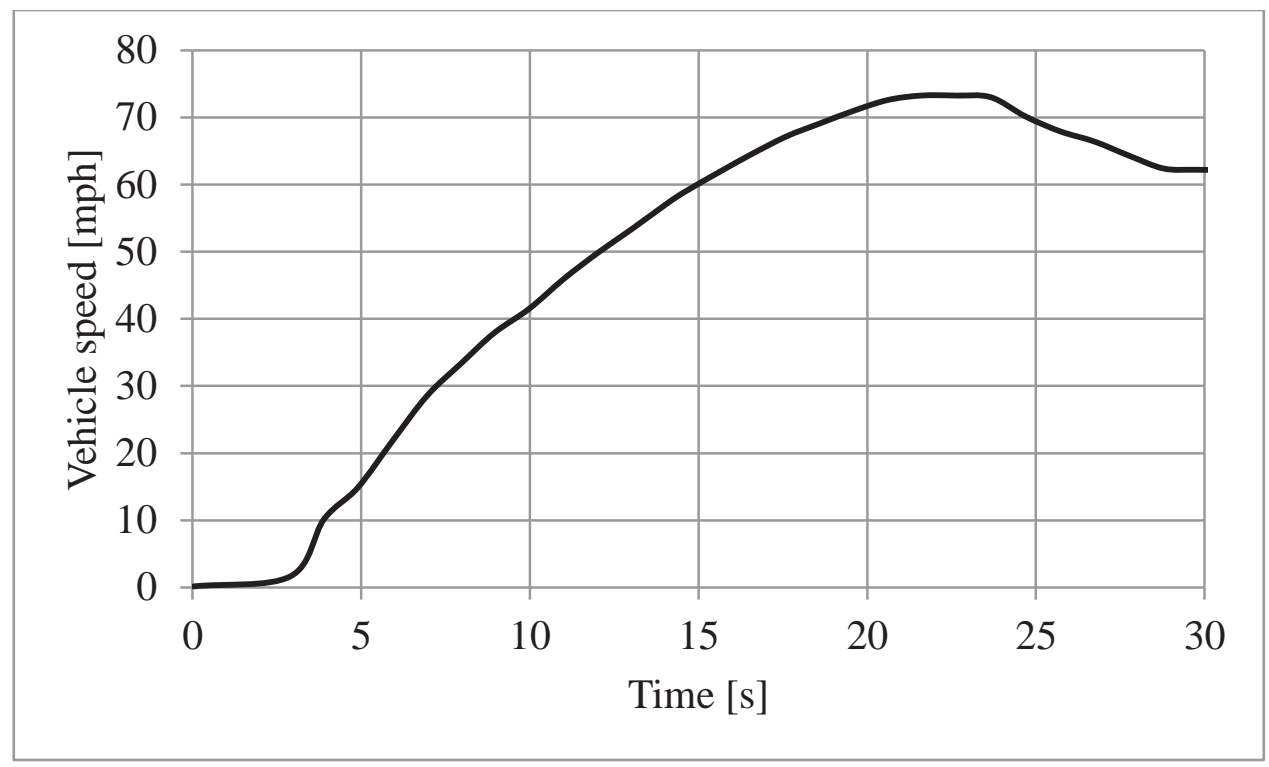

Figure 9-21: Acceleration test results.

Acceleration and braking tests were also completed for both the model and the actual vehicle. Both were tested for 0-60 mph acceleration, 50-70 mph acceleration, and braking distance from 60-0 mph. Table 9-4 displays the performance test results for the actual vehicle to the PSAT model.

Table 9-4: Performance comparison of on-road test results to the PSAT model.

\begin{tabular}{|l|c|c|c|}
\hline & PSAT & Actual & Difference \\
\hline $0-60$ mph (s) & 14.6 & 16.0 & $9.6 \%$ \\
\hline $50-70$ mph (s) & 8.6 & 10.0 & $16.3 \%$ \\
\hline Braking Distance (ft) & 150.0 & 148.5 & $0.1 \%$ \\
\hline
\end{tabular}

\subsection{5 $\quad$ Towing Test}

The goal of the towing test was to demonstrate towing ability at a constant 45 mph speed up a 3.5\% grade for 15 miles, as shown in Figure 9-22. This drive schedule 
tested real-world conditions that the production vehicle may experience. This effectively tested the vehicle's ability to consistently pull a 1,500 pound load up a hill.

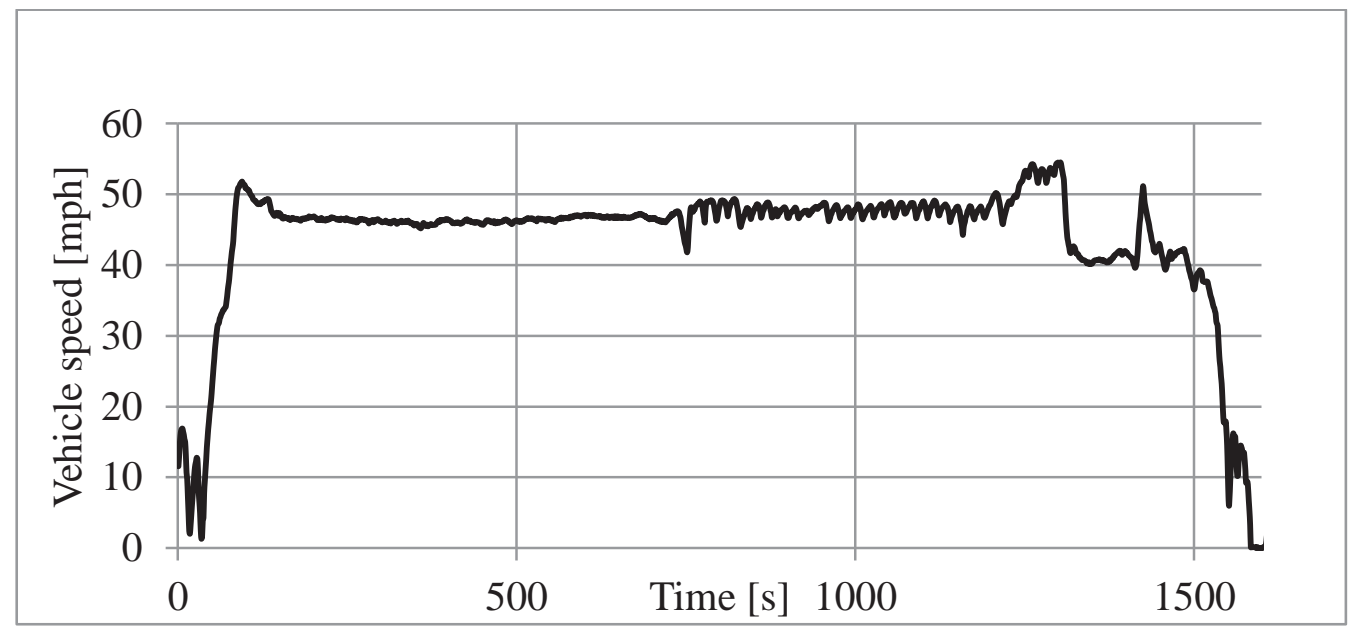

Figure 9-22: Towing test cycle.

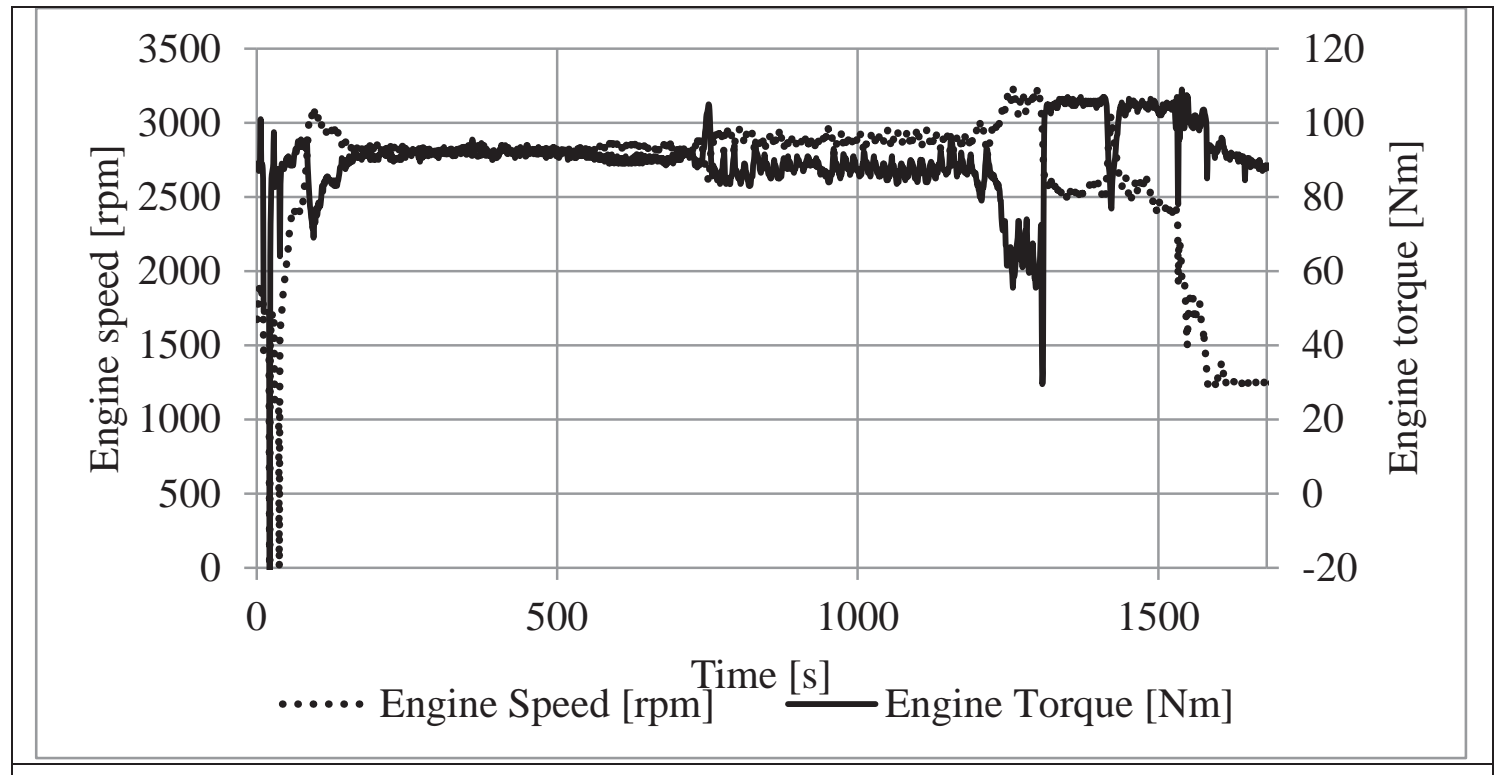

Figure 9-23: Towing test engine operation vs. time. 


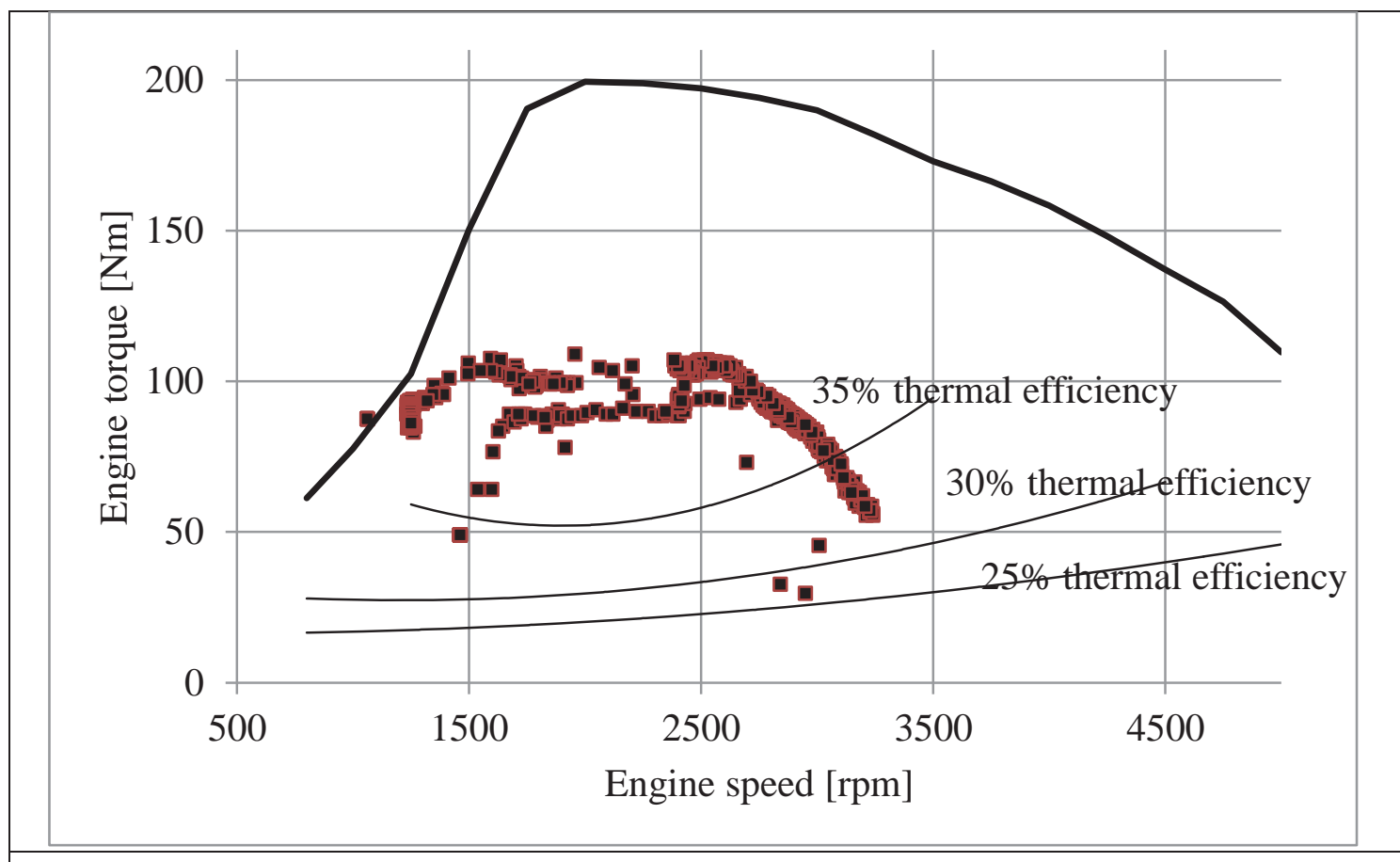

Figure 9-24: Towing test engine operation vs. efficiency.

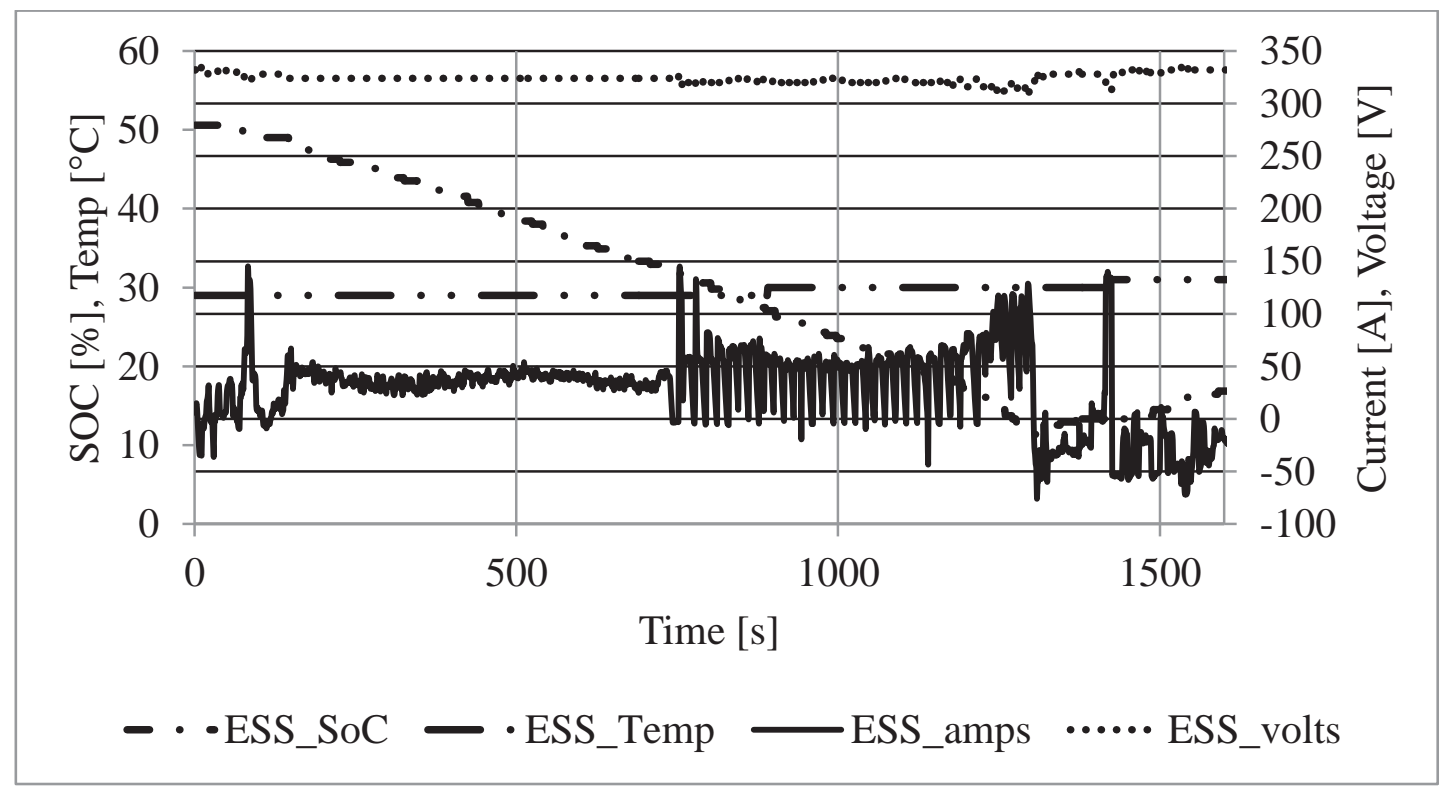

Figure 9-25: Towing test high voltage battery operation. 
The goal of the combined city \& highway test 1 was to evaluate the vehicle's operation over long time duration on city and highway drive schedules. The combined city \& highway test 1 evaluated the vehicle's operation for approximately 75 minutes/38 miles with stop-start, city and highway conditions, as shown in Figure 9-26. The average speed and maximum speed of the combined city \& highway test 1 were 27 mph and 63 mph.



Figure 9-26: Combined city and highway test cycle 1.

The Engine operating diagram displayed the engine's operation during the combined city \& highway test 1, shown in Figure 9-27 and Figure 9-28. The engine was on all the time during the test. The engine speed operated between 1,000 rpm to 3,660 rpm and engine torque between 0 to $124 \mathrm{Nm}$. The average engine speed and torque were 2,150 rpm and $61 \mathrm{Nm}$. From the statistical results, the engine operated 55\% of the time in the efficiency area above $35 \%$ and $68 \%$ of the time in the efficiency area above $30 \%$. 


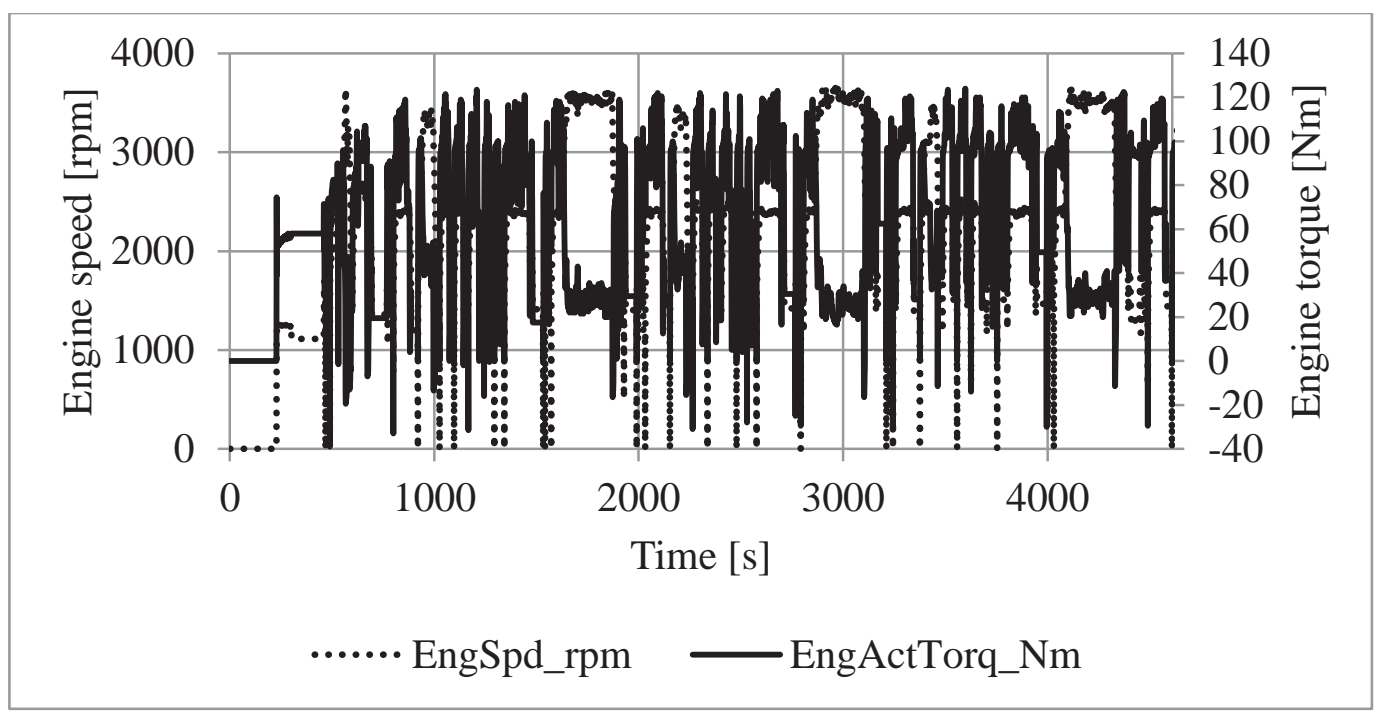

Figure 9-27: Combined City \& Highway Test 1 engine operation vs. time.

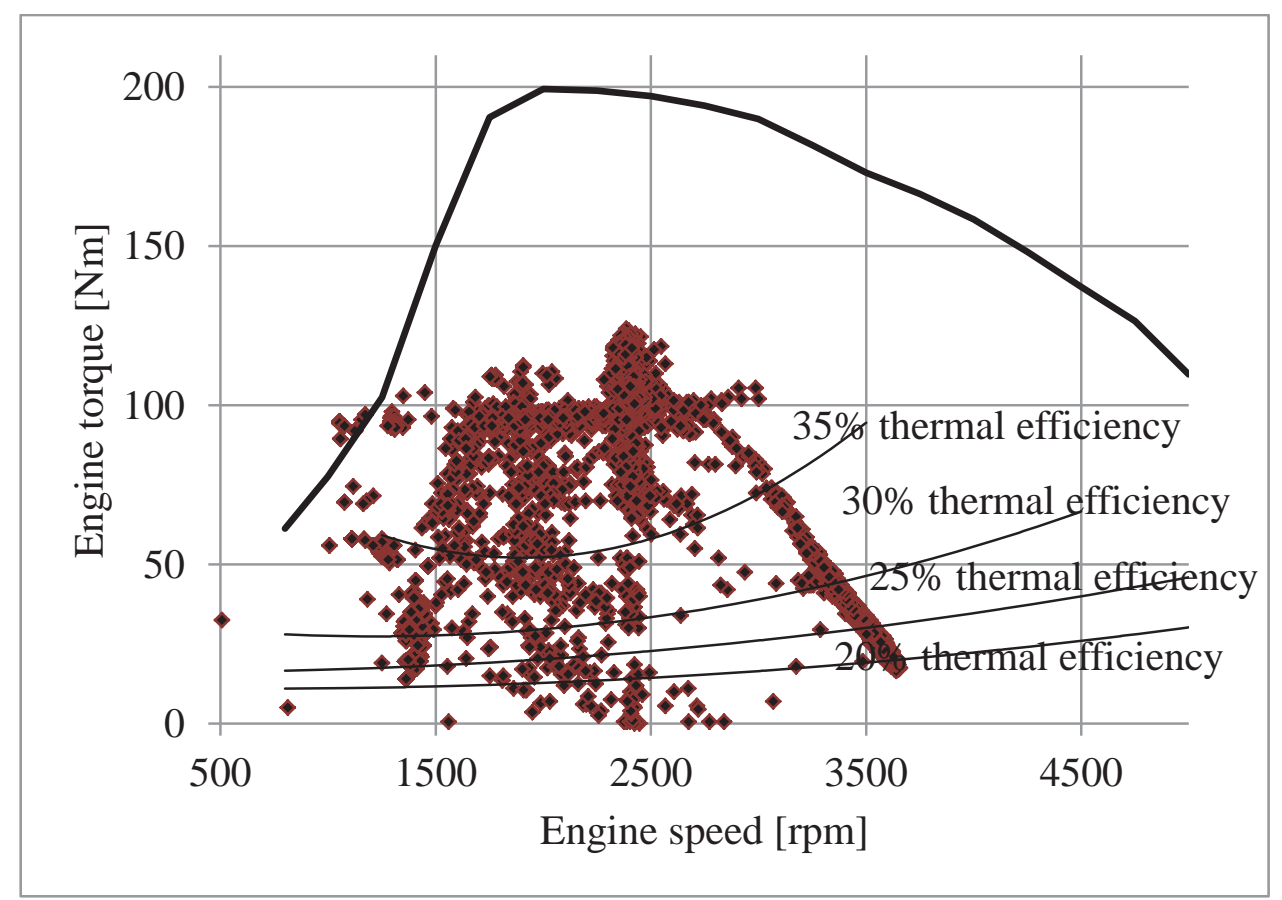

Figure 9-28: Combined City \& Highway Test 1 engine operation vs. engine efficiency.

The HV battery diagram displayed the battery's operation during the combined city \& highway test 1 , shown in Figure 9-29. The battery temperature increased from 30 ${ }^{\circ} \mathrm{C}$ to about $33{ }^{\circ} \mathrm{C}$. The battery SOC dropped from $60 \%$ to $42 \%$. The battery pack voltage 
was around $330 \mathrm{~V}$ during the test. From the statistic result, the maximum and average discharging current were $143 \mathrm{~A}$ and $33 \mathrm{~A}$ respectively. The maximum and average charging current were -77 A and -30 A respectively. The battery conducted 52\% discharging operation and $42 \%$ charging operation.

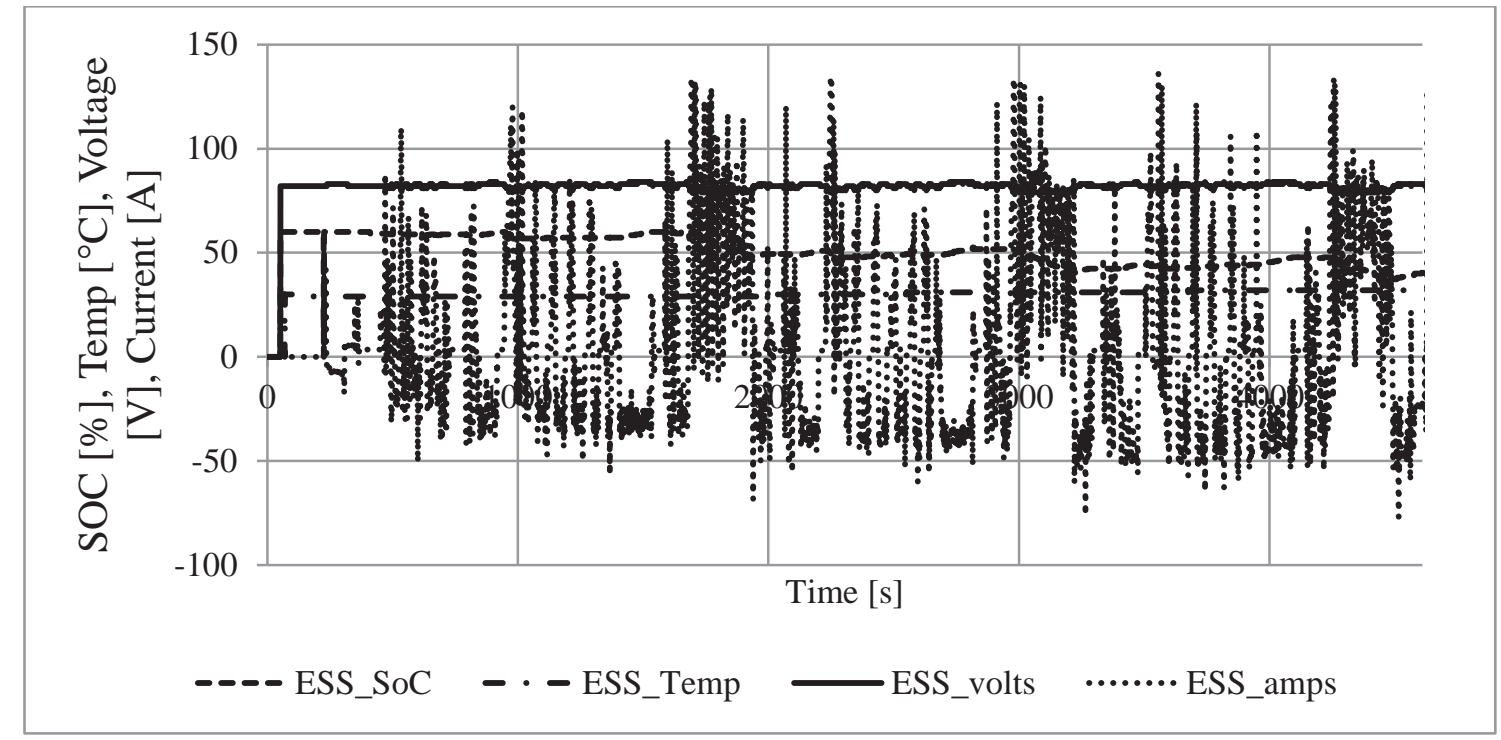

Figure 9-29: Combined City \& Highway Test 1 high voltage battery operation.

\subsubsection{Combined City \& Highway Test 2}

The combined city \& highway test 2 evaluated the vehicle's control specification in a city and highway combined cycle, as shown in Figure 9-30. The combined city \& highway test 2 evaluated the vehicle's operation for approximately 20 minutes/10 miles with stop-start, city and highway conditions. The average speed and maximum speed of combined city \& highway test 2 were $31 \mathrm{mph}$ and $63 \mathrm{mph}$. 


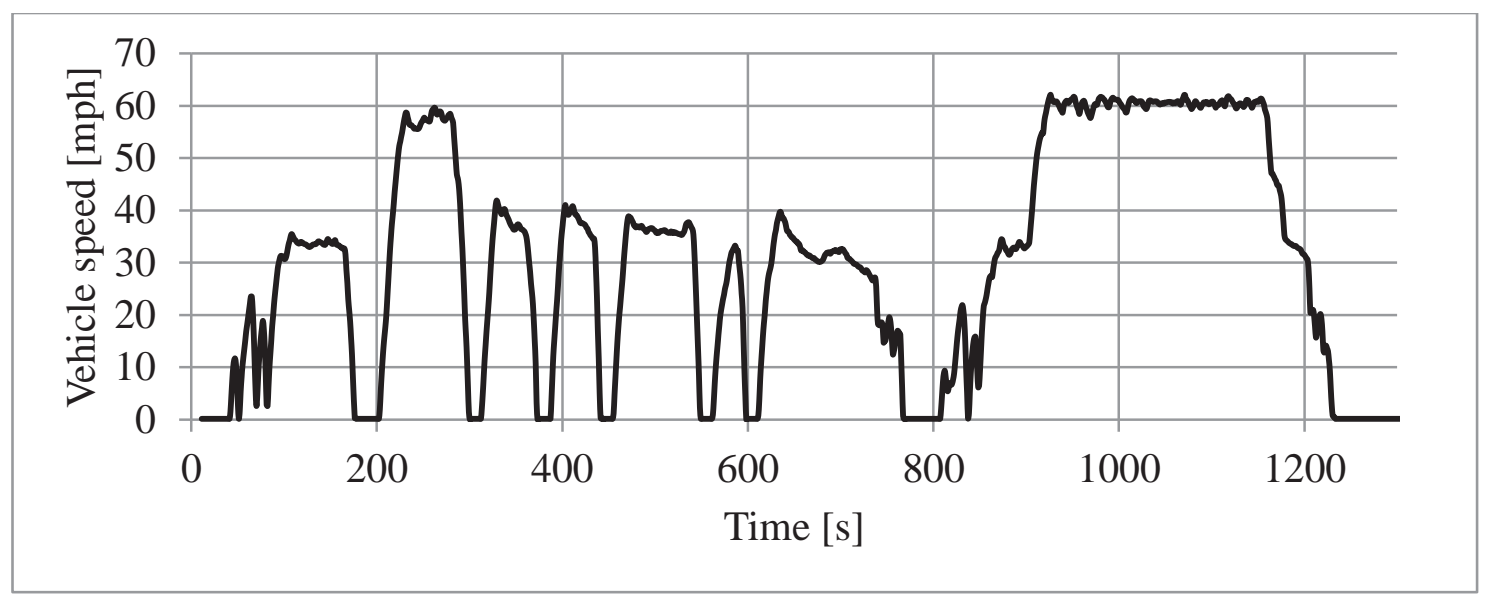

Figure 9-30: Combined city and highway test cycle 2.

The engine operating diagram displayed the engine's operation during the combined city \& highway test 2, shown in Figure 9-31 and Figure 9-32. The engine speed operated between 1,000 rpm to 3,600 rpm and engine torque between 0 to $123 \mathrm{Nm}$. The average engine speed and torque were 2,370 rpm and $73 \mathrm{Nm}$. From the statistic result, the engine operated $46 \%$ of the time in the efficiency area above $35 \%$ and $56 \%$ of the time in the efficiency area above $30 \%$.

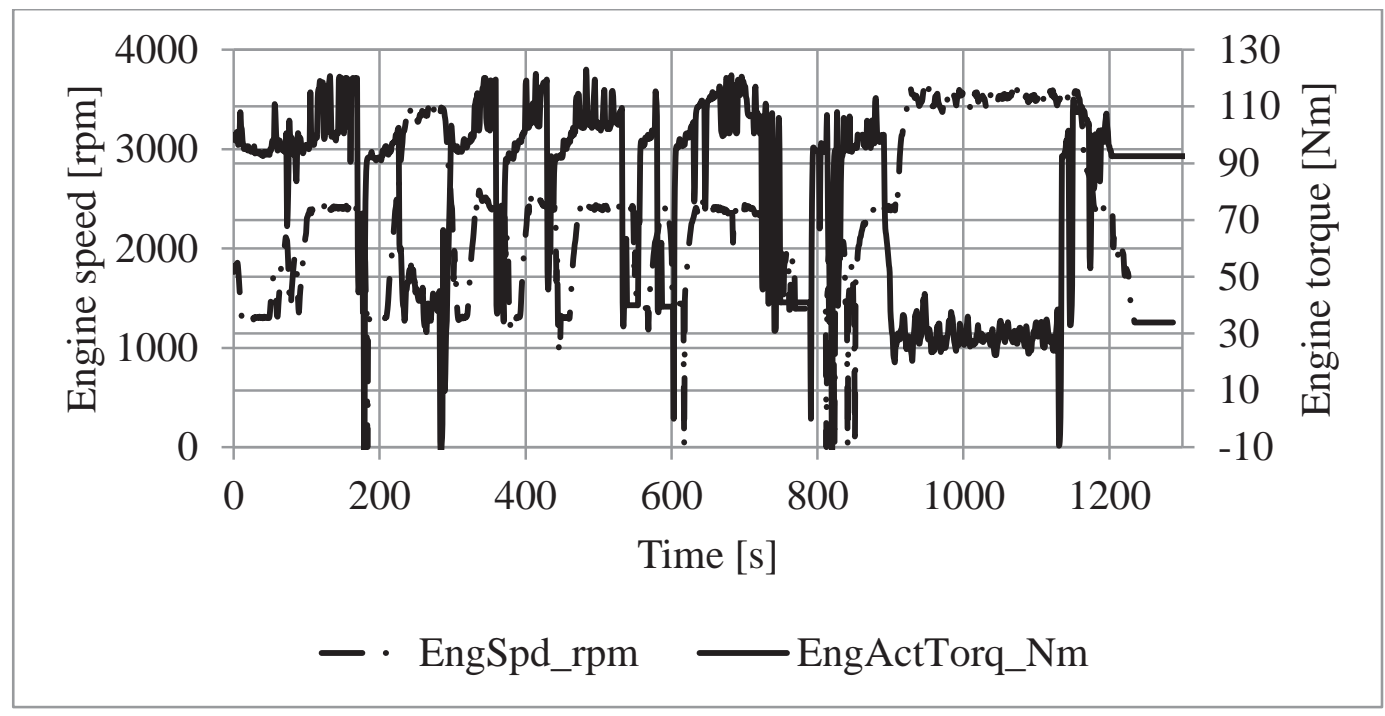

Figure 9-31: Combined City \& Highway Test 2 engine operation vs. time. 


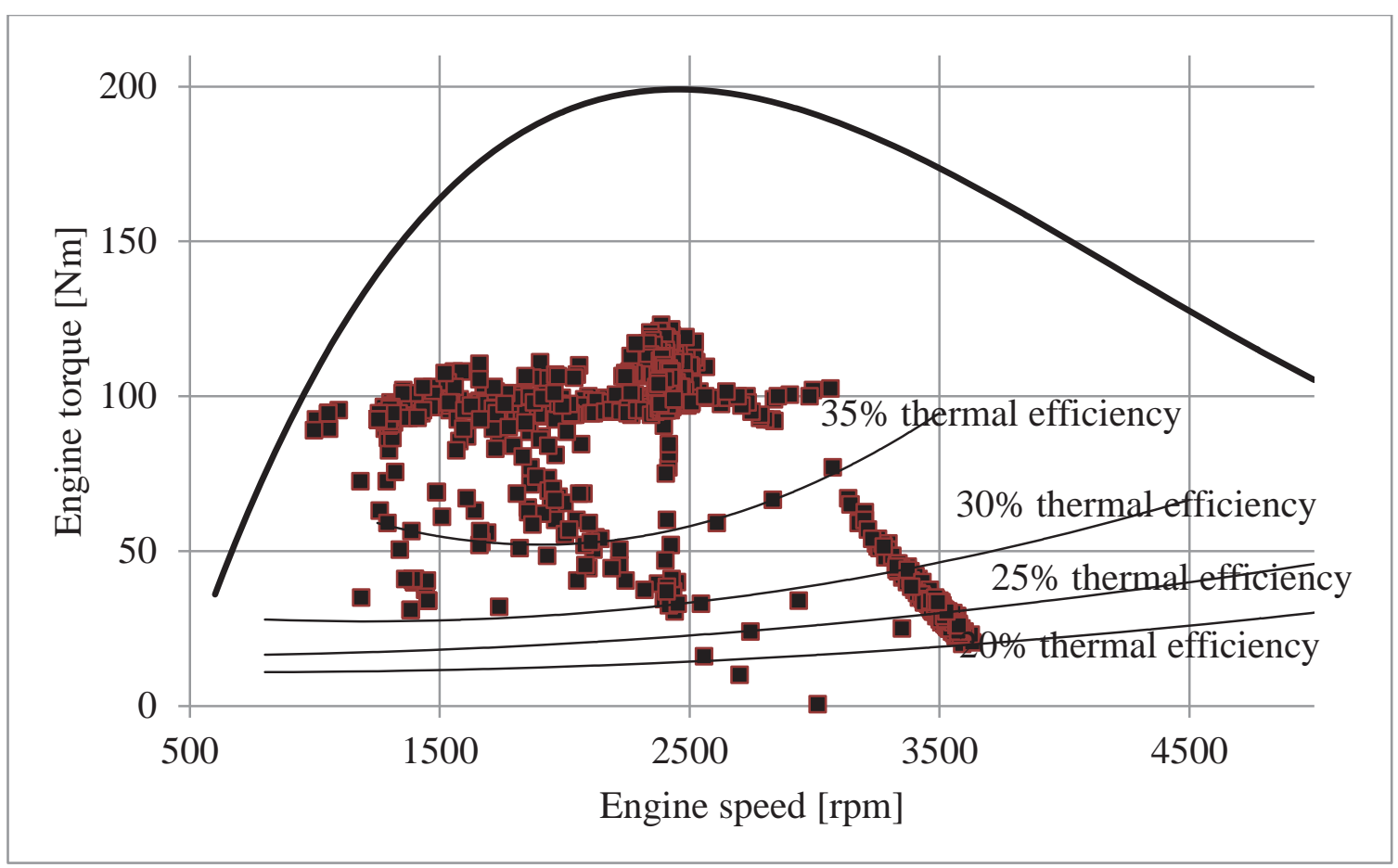

Figure 9-32: Combined City \& Highway Test 2 engine operation vs. engine efficiency.

The HV battery diagram displayed the battery's operation during the combined city \& highway test 2, as shown in Figure 9-33. The battery temperature increased from $33{ }^{\circ} \mathrm{C}$ to about $35^{\circ} \mathrm{C}$. The battery SOC was sustained at $36 \%$. The battery pack voltage was around $330 \mathrm{~V}$ during the test. From the statistical results, the maximum and average discharging current were $140 \mathrm{~A}$ and 44 A respectively. The maximum and average charging current were -76 A and -33 A respectively. The battery conducted 27\% discharging operation and 39\% charging operation. 


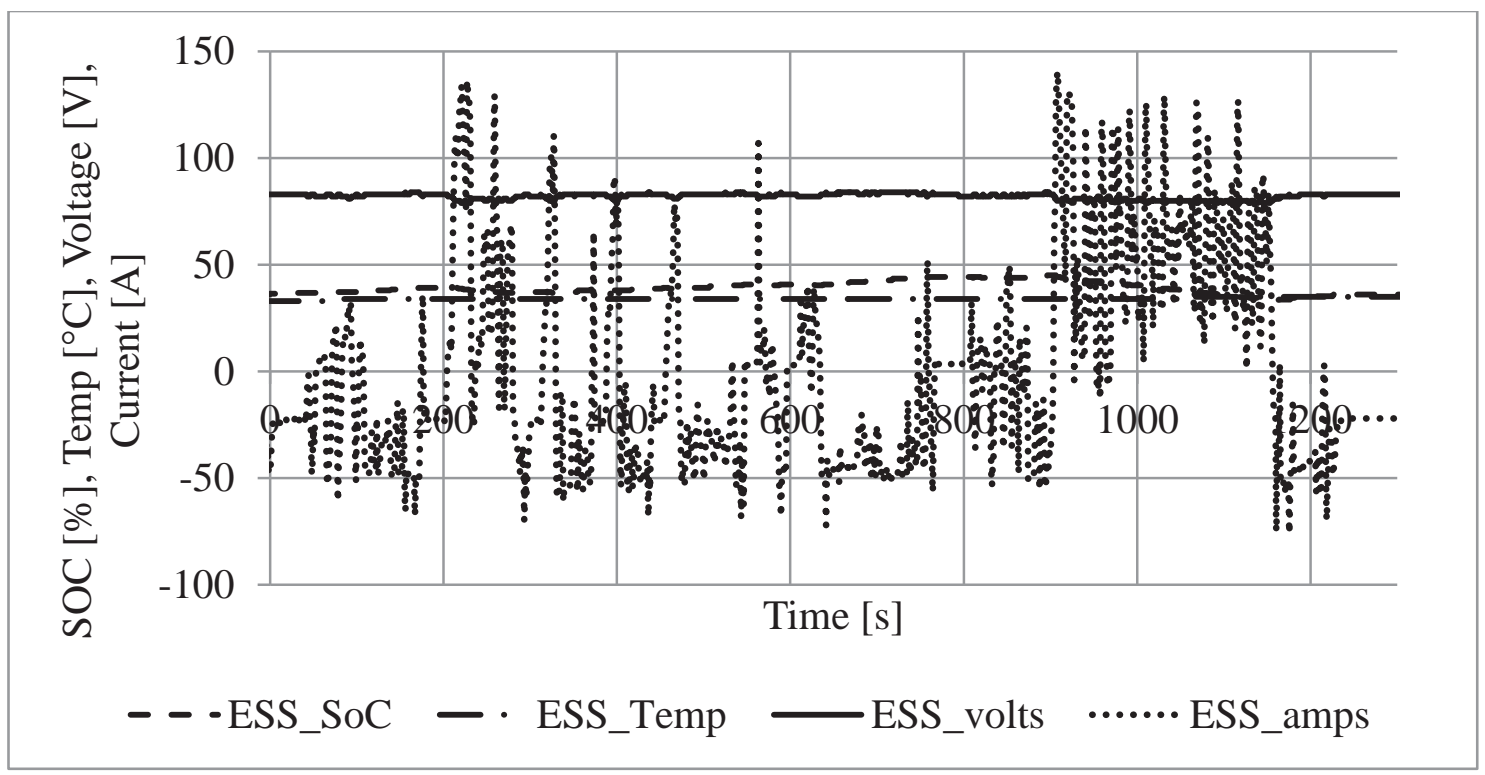

Figure 9-33: Combined City \& Highway Test 2 high voltage battery operation.

\subsection{Summary}

The GM 2-Mode transmission combined the strength and load capacity of conventional automatic transmission with the increased fuel economy provided by the hybrid-electric vehicle architecture. The 2-Mode hybrid control algorithm was implemented to include the hybrid functions of engine stop-start, two EVT transmission modes, regenerative braking and high voltage battery SOC sustaining operation. The prototype HEV was fully functional and street legal. It achieved a 10 MPG improvement in combined fuel economy compared to a base model 2009 Saturn Vue while maintaining acceptable performance characteristics. By implementing a more in-depth engine and transmission shifting control algorithm, the vehicle's fuel efficiency could be further improved over the baseline. Simulation results using PSAT indicated fuel economy of 28.4/29.4 MPGge over the MUDS/ R19HW, 14.6 s 0-60 mph acceleration time and $8.6 \mathrm{~s}$ 50-70 mph acceleration time. The on-road test results indicated fuel economy of 
24.5/31.5 MPGge over the MUDS/R19HW cycles, 16 s 0-60 mph acceleration time and 10 s 50-70 mph acceleration time. 


\section{CHAPTER 10}

\section{CONCLUSIONS AND RECOMMENDATIONS}

The demand of the petroleum fuel in automotive transportation will be even higher due to rapidly increasing vehicle population in the world. Petroleum fuel consumed in automotive transportation pollutes the air with harmful tailpipe emissions. It is widely agreed that greenhouse gases emission cause global weather changes. Hybrid vehicle technologies provide an alternative to reduce the automotive transportation dependence on the petroleum fuel.

The overall goal of the research was to develop and test a hybrid electric powertrain with improvement in fuel economy while maintaining the vehicle's performance. The GHGs emission will be reduced accordingly with less petroleum fuel consumption.

\subsection{Statement of Work}

The 2-Mode hybrid control algorithm research work started with the hybrid powertrain simulation with PSAT software. The simulation results projected equal performance and a fuel economy improvement of $40 \%$. The proposed hybrid powertrain was integrated into a stock 2009 Saturn Vue. The rule-based hybrid control algorithms were applied in two real-time controllers to achieve the hybrid powertrain control functions, including engine stop-start, engine optimal speed/torque operation, transmission shifting, regenerative braking and HV battery SOC sustaining operation. The prototype HEV was expected to operate with EV drive, regenerative braking, two EVT modes, and engine stop-start and optimal speed/torque operations. The prototype 
HEV was tested on road to evaluate the fuel economy and the performance. The on-road test results were also used to validate the simulation model for accuracy and reliability.

\subsection{1 $\quad$ Hybrid Electric Vehicle Simulation}

The hybrid powertrain built in the simulation model can provide similar quality as the real powertrain integrated in the vehicle if the model is developed accurately enough. PSAT is designed to meet the requirements of automobile engineering to design hybrid powertrains, from the plants to the controls.

Based on the HEV literature review, the candidate HEV architectures were simulated to select the best hybrid powertrain architecture based on the selection criteria to represent the research focus. The simulation results provided predictions of vehicle performance and fuel economy for the candidate HEV architectures.

For the selected 2-Mode hybrid powertrain, a rule-based hybrid control algorithm was implemented in the simulation and applied in the HEV to control the hybrid powertrain. To verify the accuracy of the 2-Mode PSAT model, the fuel economy and vehicle performance in the simulation results were validated with the HEV on-road test results by using the same driving cycles. The operation specifications of the powertrain components, such as engine, transmission, high voltage battery and the electric motors, were compared to validate the PSAT model. The local city and highway test cycles, MUDS and R19HW, were created and used to evaluate the PSAT model simulation results and the on-road test results. The MUDS and R19HW were also used to evaluate the HEV's calibrations to reach the best combination of fuel economy and performance.

The 2-Mode HEV vehicle was modeled using a quasi-static method on the vehicle longitudinal behavior. The driver was modeled with the driver's pedal response over the 
speed profile of the drive cycles. The engine was modeled on the request of the engine speed and torque. The 2-Mode transmission model was built on the transmission dynamic model. The high voltage battery model was built on the empirical test data and the request of the charging and discharging power. The UDDS, HWFET, US06 Supplemental Federal Test Procedure (SFTP), Morgantown_City, Morgantown_Highway and acceleration cycles were simulated to evaluate the performance and fuel economy. WTW PEU, WTW GHS, and other tailpipe emissions were evaluated in GREET. The simulation results indicated a significant fuel economy improvement over 30\%.

The simulation results indicated a CAFE unadjusted fuel economy of 33/35 miles per gallon gas equivalent (MPGge) in UDDS/ HWFET. WTW GHGs emissions were then computed using GREET and PEU was computed based on WTP PEU factors determined by the default allocations for 2015 in GREET. The prototype hybrid vehicle generated less $(150 \mathrm{~g} / \mathrm{km})$ WTW GHG emissions and consumed less $(0.4 \mathrm{kWh} / \mathrm{km})$ petroleum energy while maintaining an 11 s 0-60 acceleration time and $680 \mathrm{~kg}$ (1,500 lbs) towing capacity based on simulation results. Passenger capacity, cargo capacity and utility were not sacrificed by addition of the ESS pack and hybrid propulsion systems. The engine was fueled on a blend of $20 \%$ biodiesel derived from plant oil and $80 \%$ petroleum-derived ultra-low sulfur diesel fuel (B20). GREET indicated that the $1.3 \mathrm{~L}$ diesel engine fueled on a B20 biodiesel blend achieved high TTW fuel economy and the lowest GHG emissions.

\subsubsection{Hybrid Powertrain Architecture Selection}

The hybrid powertrain architecture selection was introduced as the background of the research. Based on the literature review, the candidate hybrid powertrain architectures 
were modeled in PSAT software to project the vehicle's performance, fuel economy and GHGs emission.

In order to select the best hybrid architecture, a selection criteria and a scoring method were created to reflect the research focus on the acceleration performance, the combined CAFE fuel economy, GHGs emission, the drive range and the energy usage. The selected 2-Mode hybrid powertrain had the best combined fuel economy, performance and greenhouse gases emission according to the selection criteria and PSAT simulation results.

\section{2-Mode Hybrid Control Algorithm Development}

The power split device applied in electrically variable transmission was analyzed and the dynamic specification was studied for smooth control and efficiency improvement of the hybrid control algorithm. The power split device working theory was explored to analyze the operation efficiency. Input split and compound split (2-Mode) transmissions were further studied to compare the advantages and the disadvantages considering the operating efficiency and the control complexity. The 2-Mode transmission EVT mode operating limits were analyzed due to the speed ranges of the engine and the electric motors. The kinematics of the 2-Mode transmission were analyzed with mathematical equations and the lever diagram. The transmission shifting control was presented for efficiency and performance considerations.

A rule-based control method has been widely used in the hybrid vehicle control systems because it provides easy solutions for complex hybrid powertrain control and can achieve optimal control results. The overall hybrid control architecture was presented to understand the high level hybrid control process. The hybrid powertrain control 
algorithms were discussed in detail, including engine stop-start, 2-Mode transmission shifting, HV battery SOC control, optimal engine speed and torque control, vehicle propelling torque and regenerative braking control.

Three main targets were set during the rule-based hybrid control algorithm development. The first target was to maximize the vehicle hybrid torque to improve or maintain the HEV performance since the engine was downsized from a 3.6 L gasoline engine to a $1.3 \mathrm{~L}$ diesel engine. The second target was to maximize the fuel economy by optimizing the engine operation since the engine provided the average power to drive the vehicle. The third target was to sustain the HV battery SOC in all driving cycles. In order to realize the designed hybrid control system targets, the related hybrid control functions included engine stop-start control, engine optimal speed/torque control, hybrid transmission shifting control, the HV battery SOC balance control, regenerative braking control and vehicle propelling torque control. The engine provided the average power to drive the vehicle since the HEV operated with a HV battery SOC sustaining control algorithm. The HV battery SOC was sustained at 50\% considering the battery technical specifications. Regenerative braking torque was commanded to capture as much braking energy as possible, taking into account the electrical system critical safety concerns, such as overcharging, over-discharging, overheating and over current flow. The vehicle propulsion torque demand was controlled to deliver the maximum powertrain capability to ensure the best performance by combining the power sources from both the engine and the HV battery.

Based on the hybrid control program derived from the rule-based control method, calibrations were made to optimize the control program to maximize the performance and 
the fuel economy. The fuel economy target was achieved by testing the related hybrid control functions, such as regenerative braking control, transmission shifting control and engine stop-start control. Regenerative braking function was turned on or off to study how much it affected the fuel economy on both city and highway driving cycle. The transmission's two EVT modes could be shifted at different vehicle speeds. EVT mode shift strategies were examined to evaluate how much it affected the fuel economy on both city and highway driving cycles. In the hybrid control program, the engine could be shut down at different vehicle speeds. Vehicle fuel economy was examined with the engine always on and with the engine shut down with different control strategies.

Vehicle performance was evaluated with different hybrid control calibrations. The HEV performance was a critical concern since the engine was downsized from a 3.6 L gasoline engine to a $1.3 \mathrm{~L}$ turbo diesel engine. Acceleration capability was a typical parameter to evaluate the vehicle performance. Research was focused on how much the HV battery SOC level and different transmission shifting algorithms affected the HEV acceleration capability. Transmission shifting between the two EVT modes changes the transmission speed ratios, which can affect vehicle acceleration capability. Different transmission shifting calibrations at different vehicle speeds were studied to determine how it affected the vehicle acceleration. The prototype HEV was tested for its acceleration performance, from 0-60 mph and 50-70 mph.

The target HV battery SOC was a challenge for the hybrid control algorithm. The SOC sustaining control program is not a standalone system. The SOC sustaining control is affected by the regenerative braking function, the engine stop-start function, the engine power command and the complicated road load conditions. Extra power needed for 
sustaining the SOC at the target will be commanded from the engine if regenerative braking cannot charge the battery enough. A HV battery SOC Proportional-Integral (PI) controller was implemented to sustain the SOC. On-road tests with different test cycles were done to see if the program could effectively sustain the SOC. The HV battery drives the electric motor(s) to help the engine to boost the vehicle power. Research was done to evaluate how different HV battery SOC target levels affected vehicle performance and the fuel economy.

\subsubsection{Hybrid Control System Implementation}

The 2-Mode hybrid powertrain control system consisted of strategic selection, tactical selection and hand-to-hand execution. The strategic selection and the tactical selection were within the range of the hybrid control system optimization, and thus were studied to improve vehicle performance and fuel economy. Strategic selection involved the transmission shifting logic and engine stop-start control. Only the two EVT modes were explored in this dissertation. Fixed gear operations were not investigated due to the sophistication involved in control. Mode 1 was used for the low speeds, and the engine could be either on or off. Mode 2 was used for the high speed range, but the engine must be on. When the vehicle speed was high, the engine was turned on to provide extra power. When the high voltage SOC was low, the engine was turned on at low power to charge the battery. Tactical selection involved controlling the optimal engine speed and torque. The engine speed command followed the vehicle speed to increase the engine power output. The engine torque provided as much power as possible to achieve the axle torque command and used less battery power when high voltage SOC was low. The TCM performed the shifting command. The DECM executed engine stop-start, and responded 
to the optimal engine speed and torque requests. The TPIM commanded both the torque and speed of the electric motors.

The rule-based hybrid control algorithm was applied to design and implement the hybrid supervisory control algorithm, including strategic selection and tactical selection. The supervisory hybrid control system was a complex multiple input and multiple output (MIMO) system. After simulation and analysis of the hybrid powertrain components, a rule-based hybrid control program provided a feasible alternative to reach an optimal solution. Rule-based control logic was also implemented in the Toyota Hybrid System and achieved desired results [80]. The rule-based control strategy was developed based on the 2-Mode transmission dynamic model and the analysis of the specifications of the engine and the ESS.

The hybrid control architecture was based on the ability to quickly and accurately respond to the driver's torque demand via the accelerator and brake pedal positions. The accelerator pedal or brake pedal signal was sent to the SCU where, based on the depression percentage and the current velocity of the vehicle, an output propelling or braking axle torque was generated. The axle torque was positive for propelling and negative for braking. The SCU regulated the power distribution of the vehicle, meaning that it decided the percentages of power that came from both the engine and/or the battery system. It also determined desired engine stop-start status, the vehicle driving mode as propelling and braking and relayed this information to the TPIM. Hybrid transmission mode selection was a key part to realize the desired fuel economy and performance. The ACU handled the interface with the ESS. 
The hybrid control system was implemented in the prototype 2-Mode HEV to achieve the designated control functions. The hybrid control algorithm was implemented in two real-time system controllers. Engine stop-start was achieved by control the ignition pin in the DECM. Engine torque was commanded by using a PWM of voltage to Analogue module to convert the Supervisory Control Unit (SCU) engine torque command to the voltage outputs, which emulated the potentiometer sensor signal from the acceleration pedal. A HVIL circuit was implemented in the electrical system to improve the HEV safety specification. The HV battery main contactors close/open control monitored the ignition key status, HVIL status, vehicle fault status, ground fault status and the TPIM status. The HV battery discharging and charging power fold-back was applied by monitoring the HV battery temperature. A new high speed CAN bus was added for the DECM and the battery management system (BMS) to avoid message identification (ID) conflicts.

The prototype hybrid powertrain was built and installed in a 2009 Saturn Vue. The 1.3 L diesel engine took the place of the stock 3.6 L gasoline engine under the hood. The high voltage battery was located under the rear cargo area floor. The diesel engine harness was wired to the vehicle stock harness to control the engine stop-start, fuel pump and to deliver the optimal engine torque. The high voltage battery harness was wired with the stock harness. The CAN integration of the diesel engine and the high voltage battery with the stock control units was realized by adding an extra high speed CAN channel, West Virginia High Speed CAN (WVLAN). The diesel engine CAN communication emulated the messages of the gasoline engine. The high voltage battery CAN communication included the high voltage battery status updates and the vehicle hybrid 
function requests. Two controllers, SCU and ACU, were integrated with the stock control units to achieve the hybrid control functions. The SCU executed the main vehicle hybrid functions, such as engine stop-start, hybrid transmission mode selection, regenerative braking and engine operation in high efficiency zones. The ACU handled the CAN communication between the high voltage battery and the TPIM. The ACU also executed the safety check process before energizing the high voltage system.

The most significant electrical work related to hybrid powertrain control included the harness design of the Diesel Engine Control Module (DECM) and the high voltage BMS. The DECM was wired to control the engine stop-start, fuel pump and to deliver the optimal engine torque. The BMS was wired to integrate the HVIL circuit and to set different operation modes. The BMS had two working modes, vehicle mode and charging mode.

The Controller Area Network (CAN) made the use of a real-time distributed control system possible. An extra high speed CAN bus, WVLAN, was added for the communication with the diesel engine controller and the high voltage battery BMS. CAN ID conflicts with the original two high speed CAN buses, GMLAN and PTEB, were eliminated by adding the WVLAN.

\subsubsection{Vehicle Testing}

The vehicle and the hybrid powertrain components were tested during the vehicle development phase and the on-road tests. The diesel engine was tested to verify the engine's basic setup of the DECM harness and the CAN communications. The engine output torque was calibrated responding to the accelerator pedal inputs. The high voltage battery was set on a bench to test the communication and the basic control specifications. 
During the vehicle development, most of the hybrid powertrain information was monitored with the extensive data log system developed on VECTOR CANcaseXL log. The VECTOR CANcaseXL log provided the solution for online CAN message monitoring and offline data analysis. The Woodward MotoTron controller provided the solution for on-board diagnostics and control algorithm monitoring to ensure the proper function of the control strategy.

Once the hybrid powertrain components were verified out of the vehicle, they were integrated into the vehicle and tested. First, the proper communication between the hybrid powertrain component and the stock control units was verified. Then the vehicle was tested while stationary for functions. The vehicle was tested under different driving conditions. Once all these functions were verified, the control strategy was optimized to improve the vehicle energy efficiency and to maintain the performance.

The test results were used to validate the HEV model developed in PSAT. The hybrid control program was calibrated to achieve the best tradeoff between the fuel economy and performance. Two local test cycles, the MUDS and R19HW, were created for on-road test to evaluate the vehicle performance and the fuel economy. Then the MUDS and R19HW cycle profiles were introduced in the PSAT model to compare with the simulation results, which were validated with the on-road test results. The powertrain components operations were further compared to validate the HEV PSAT model.

\subsection{Contributions and Conclusions}

The contributions of this research were as follows:

The front wheel drive 2-Mode hybrid powertrain with a turbo diesel engine demonstrated an alternative to sustain petroleum energy and reduce the petroleum oil 
dependence in automotive transportation. The prototype HEV had the combined fuel economy at 28.4 MPGge with the MUDS/R19HW test cycles, which was 24.0\% higher than the mule vehicle. The 2-Mode prototype HEV provided the research platform to learn how it can improve the vehicle's fuel economy in the real world. The 2-Mode HEV provided an option for a mid-size CUV/SUV to achieve higher fuel economy to meet more strict fuel economy regulation started from 2016 by EPA.

The novel 2-Mode HEV PSAT model was validated with the vehicle on-road test data and used to analyze the performance and fuel economy of the proposed hybrid powertrain. No research had been done previously to develop a FWD 2-Mode PSAT model and validate it with the on-road test data. The research demonstrated the validation of the PSAT powertrain component models and the control strategy using the HEV onroad test data. The validated PSAT model provided a research platform to further study the novel 2-Mode powertrain.

The 2-Mode hybrid powertrain advantages and disadvantages were analyzed as a result of the thorough comparison with the 1-mode transmission. The structure of 1-Mode and 2-Mode was analyzed. The lever diagram was introduced to analyze the power split device and the related HEVs. The lever diagram analyzed the vehicle drive under different scenarios. The 2-Mode is more efficient at high speed than the 1-Mode, which was analyzed with hybrid powertrain efficiency equations and plots.

\subsection{Future Work and Recommendations}

The 2-Mode hybrid electric vehicle was proved to improve the fuel economy by $24 \%$ percent while maintaining most vehicle performance, except for the acceleration. The rule based hybrid control algorithm was capable of achieving the designated control 
functions. The calibration effort has been made to get the maximum output from the vehicle. However, different control rules could be applied to check if there is more room for fuel economy improvement. In the future, another concern of the hybrid control system is safety, such as lost CAN communication among hybrid powertrain components.

Different engine control rules can be applied to further improve the vehicle fuel economy and examine how much the vehicle performance will be sacrificed. The 2-Mode hybrid control system applied a high voltage battery SOC sustaining strategy, so eventually the engine provided the average amount of power and energy to drive the vehicle. From the towing test results, it can be found that the engine was operating above $35 \%$ efficiency over $80 \%$ of time when the engine was operating with higher torque output. The vehicle's fuel economy could be further improved if the engine efficiency is improved. Engine optimal operation line for the system control can be applied to further increase the engine operating efficiency [81, 82]. Based on the on-road test results, the engine optimal operation line can be redefined to further improve the engine operation efficiency. When the engine operating points are in the low-efficiency area, the efficiency can be improved by commanding extra engine torque. However, the extra engine power will be used to charge high voltage battery. Hence, high voltage battery SOC needs to be monitored closely to avoid unintended charging.

HIL simulation should be used to validate the hybrid control system and reduce the on road testing. HIL test has been proven to be an effective tool to assist in the vehicle control system development. The 2-Mode model built with PSAT was based on the Simulink software, which can be extracted for the HIL application for both the hybrid 
control program and the plant model. The standard mid-size dSPACE HIL will simulate the 2-Mode HEV plant model, and the 2-Mode real-time system controllers will interface with the HIL using CAN and IO channels. The HIL setup is able to validate the hybrid system control functions and apply IO faults and communication faults during the test. This would help development of an accurate 2-Mode transmission model.

Fault Tree Analysis (FTA) and Design Fault Mode and Effect Analysis (DFMEA) methods should be applied and used to test the hybrid controller in the HIL to improve the safety features of the hybrid control algorithm. Vehicle control system safety is much more of a concern in an HEV. The autonomous nature of the control system can cause unintended events. The vehicle level and system level safety requirements can be defined, such as unintended acceleration/deceleration, unintended high voltage battery charge/discharge and unintended loss of acceleration/ deceleration, etc. Physical hazard analysis using FTA and FMEA should be conducted to identify hazard conditions that could arise. FTA [83] is a deductive technique which identifies the failure mode of a system according to failure modes of its subsystem and components. FTA is a failure analysis process, which begins with a hazard (top event) and ends by locating the basic component failures (basic event). This analysis method is mainly used in the field of safety engineering to identify the possibility of a safety hazard. The FTA results can be defined as the following:

- Identify critical safety components/ parts in the 2-Mode hybrid control system.

- Analysis of possible hazards within the scope of 2-Mode hybrid control system.

- Identify test cases.

- Develop HIL test plan. 
- Add mitigation plan in hybrid control system.

DFMEA [84] is an inductive technique which identifies the consequences of subsystem or component failure. The output/input matrix for the DFMEA of the 2-Mode hybrid control system should be developed to define the boundary and scope of the analysis. The matrix allows the analysis of the system to be focused on the functional requirements, components and interfaces, and their relationships. The DFMEA result should illustrate the failure modes, effects and potential causes of the 2-Mode hybrid control system.

The FTA and DFMEA results can lead to the control system test plan for critical safety concerns. Mitigation control methods will be validated by HIL to make sure that the hazards will be inhibited or reduced to an acceptable level. 


\section{REFERENCES}

1. Energy Information Administration, "International Energy Outlook 2007." Accessed June 29, 2011, http://ftp.eia.doe.gov/forecasting/0484 (2007).pdf.

2. Davis S.C., Diegel S.W. and Boundy R.G., "Transportation Energy Data Book, Edition $29^{\text {th }}$." Accessed June 29, 2011, http://cta.ornl.gov/data/tedb29/Edition29_Full_Doc.pdf.

3. Wall E., "Department of Energy (DOE) Research Strategy: Mid- and Long-Term Petroleum Savings," SAE Technical Paper 2006-21-0057, 2006.

4. Dargay J., Gately D. and Sommer M., "Vehicle Ownership and Income Growth, Worldwide: 1960-2030." Accessed June 29, 2011, http://www.econ.nyu.edu/dept/courses/gately/DGS_Vehicle\%20Ownership_2007.pdf.

5. Davis S.C., Diegel S.W. and Boundy R.G., "Transportation Energy Data Book, Edition $29^{\text {th }}$." Accessed June 29, 2011, littp://cta.ornl.gov/data/tedb29/Edition29_Full_Doc.pdf

6. Rogner H. H., "World Energy Assessment: Energy and the Challenge of Sustainability" (United Nations Development Program, Part II, Chapter 5, 2004).

7. International Energy Agency, "Carbon Capture and Storage: Progress and Next Steps." (International Energy Agency/Carbon Sequestration Leadership Forum Report to the Muskoka 2010 G8 Summit, 2010).

8. Jalil N., Kheir N.A. and Salan M., "A rule-based energy management strategy for a series hybrid vehicle.” (American Control Conference, Albuquerque, NM, Jun 4-6, 1997.)

9. Lin C.C., Huei P. and Grizzle J.W., "A stochastic control strategy for hybrid electric vehicles.” (American Control Conference, Boston, MA, June 30 -July 2, 2004).

10. Miller J. M., "Propulsion Systems for Hybrid Vehicles," London: The Institution of Engineering and Technology Press, 2006.

11. Bishop J., Nedungadi, A., Ostrowski, G., Surampudi, B., Armiroli, P. and Taspinar, E., "An Engine Start/Stop System for Improved Fuel Economy," SAE Technical Paper 200701-1777, 2007.

12. Duoba M., Lohse-Busch H., Carlson R., Bohn T. and Gurski S., "Analysis of Power split HEV Control Strategies Using Data from Several Vehicles," SAE Technical Paper 200701-0291, 2007.

13. Li X. and Williamson S. S., "Comparative Investigation of Series and Parallel Hybrid Electric Vehicle (HEV) Efficiencies Based on Comprehensive Parametric Analysis" (Vehicle Power and Propulsion Conference, IEEE, 2007).

14. Conley J., "The Role of Power and Energy Demands in Hybrid Vehicles.” Master's Thesis, West Virginia University, 2002.

15. Dean S., "Stop and start is all go for Vaeo and PSA," Automotive Engineer, 28 (11) (2003): 3/5.

16. Tamai G., Hoang T., Taylor J., Skaggs C. and Downs B., "Saturn Engine Stop-Start System with an Automatic Transmission," SAE Technical Paper 2001-01-0326, 2001.

17. Berman B., "POWER TRAIN USING MULTIPLE POWER SOURCE." Patent Identifier NO. US 3566717, March 2, 1971. 
18. Grewe T. M., Conlon B. M. and Holmes A. G., "Defining the General Motors 2- mode Hybrid Transmission." SAE Technical Paper 2007-01-0273, 2007.

19. Hendrickson J., Holmes, A. and Freiman D., "General Motors Front Wheel Drive TwoMode Hybrid Transmission." SAE Technical Paper 2009-01-0508, 2009.

20. Holmes A., Nitz L. and Kingman G., "Electrically Variable Transmissions (EVT101)" (General Motors technical presentation, 2005).

21. Chiang P. K., "2-Mode Urban Transit Hybrid Bus In-Use Fuel Economy Results from 20 Million Fleet Miles," SAE Technical Paper 2007-01-0272, 2007.

22. Chandler K. and Walkowicz K., "King County Metro Transit Hybrid Articulated Buses: Final Evaluation Results," National Renewable Energy Laboratory Technical Report NREL/TP-540-40585, 2006.

23. Wishart J. D., Zhou Y. and Dong Z. "Review, Modeling and Simulation of 2-Mode Hybrid Vehicle Architecture" (Paper presented at American Society of Mechanical Engineers (ASME) 2007 International Design Engineering Technical Conferences \& Computers and Information in Engineering Conference, Las Vegas, Nevada, September 4-7, 2007).

24. Argonne National Laboratory, "GREET Model: The Greenhouse Gases, Regulated Emissions, and Energy Use in Transportation Model.” Accessed December 1, 2013, http://greet.es.anl.gov/.

25. Rousseau A., Sharer P., Pagerit S. and Duoba M., "Integrating Data, Performing Quality Assurance, and Validating the Vehicle Model for the 2004 Prius Using PSAT," SAE Technical Paper 2006-01-0667, 2006.

26. Rousseau A., Sharer P. and Pasquier M., "Validation Process of a HEV System Analysis Model: PSAT," SAE Technical Paper 2001-01-0953, 2001.

27. Pagerit S., Rousseau A. and Sharer P., "Fuel Economy Sensitivity to Vehicle Mass for Advanced Vehicle Powertrains," SAE Technical Paper 2006-01-0665, 2006.

28. Ford, "MPG Matters: 42 Percent Say Fuel Economy Is Key in New Vehicle Purchase Decision; Influence Likely to Grow." Accessed July 1, 2013, http://media.ford.com/article_display.cfm?article_id=34825

29. U.S. Department of Energy, "How Vehicle Are Tested.” Accessed July 1, 2013, http://www.fueleconomy.gov/feg/how_tested.shtml.

30. EPA, "Emission and Fuel Economy Test Data." Accessed July 1, 2013, http://www.epa.gov/otaq/testdata.htm\#annual.

31. EPA, "EPA Federal Test Procedure.” Accessed July 1, 2013, http://www.epa.gov/nvfel/methods/ftpdds.gif.

32. EPA, "Dynamometer Drive Schedules." Accessed July 1, 2013, http://www.epa.gov/nvfel/testing/dynamometer.htm

33. Society of Automotive Engineers International, "SAE J1711 - Recommended Practice for Measuring Fuel Economy of Hybrid-Electric Vehicles," Warrendale PA, 1999.

34. Bradley T. and Davis B., "Alternative Plug in Hybrid Electric Vehicle Utility Factor," SAE Technical Paper 2011-01-0864, 2011.

35. EcoCAR Organizers, "Well To Pump Factors: Where We Got Them And How They Apply." (Presentation and Tech Talk, October 16, 2008). 
36. Argonne National Laboratory, "PSAT (Powertrain System Analysis Toolkit).” Accessed July 1, 2013, http://www.transportation.anl.gov/modeling_simulation/PSAT/.

37. Wipke K., Cuddy M., Bharathan D., Burch S., Johnson V., Markel T. and Sprik S., "ADVISOR 2.0: A Second-Generation Advanced Vehicle Simulator for Systems Analysis." (Paper presented at The North American EV \& Infrastructure Conference and Exposition, Phoenix, Arizona, Nov. 23, 1998).

38. Markel T., Brooker A., Hendricks T., Johnson V., Kelly K., Kramer B., O’Keefe M., Sprik S. and Wipke K., "ADVISOR: a systems analysis tool for advanced vehicle modeling,” Journal of Power Sources 110 (2002): 255-266.

39. Wipke K.B., Cuddy M.R. and Burch S.D., "ADVISOR 2.1: a user-friendly advanced powertrain simulation using a combined backward/forward approach," Institute of Electrical and Electronics Engineers (IEEE) Transaction on Vehicular Technology 48 (6) (1999):1751-1761.

40. Mechanical Simulation Corporation, "carSIM Mechanical Simulation.” Accessed July 17, 2013, http://www.carsim.com/products/carsim.

41. Mechanical Simulation Corporation, "Simulink, LabVIEW, and ASCET." Accessed July 17, 2013, http://www.carsim.com/products/supporting/simulink.

42. Sayers M.W., "Symbolic Computer Language for Multibody Systems," Journal of Guidance, Control, and Dynamics 14 (6) (1991): 1153-1163.

43. Mechanical Simulation Corporation, "VehicleSim Technology." Accessed July 17, 2013, http://www.carsim.com/products/supporting/vehiclesim.

44. Sun G., Wei M., Shao J. and Pei M., "Automotive Powertrain Modeling and Simulation Based on AMESim,” SAE Technical Paper 2007-01-3464, 2007.

45. Hong J., Kim S., Min B., "Drivability Development Based on CoSimulation of AMESim Vehicle Model and Simulink HCU Model for Parallel Hybrid Electric Vehicle," SAE Technical Paper 2009-01-0725, 2009.

46. Bourdon T., Saussol L. and Varoquié B., "Integration of Physical AMESim ${ }^{\circledR}$ Engine Model in Hardware in the Loop Environment, Dedicated to Engine Control Unit Testing," SAE Technical Paper 2007-01-1300, 2007.

47. LMS, “AMESim Basics.” Accessed July 17, 2013, http://www.ara.bme.hu/oktatas/tantargy/NEPTUN/BMEGEATAM05/2007-2008II/admin/amesim_english_s01.pdf

48. Gamma Technologies Inc., "GT_SUITE Overview.” Accessed June 20, 2013, http://www.gtisoft.com/products/p_GT_SUITE.php.

49. Trajkovic S., Tunestål P. and Johansson B., "Simulation of a Pneumatic Hybrid Powertrain with VVT in GT - Power and Comparison with Experimental Data." SAE Technical Paper 2009-01-1323, 2009.

50. Riegler U. G. and Bargende M., "Direct Coupled 1D/3D-CFD-Computation ( GT - Power /Star-CD) of the Flow in the Switch-Over Intake System of an 8-Cylinder SI Engine with External Exhaust Gas Recirculation,” SAE Technical Paper 2002-01-0901, 2002.

51. Zhu Y., Stobart R. and Deng J., "Analysis of the Impact on Diesel Engine Fuel Economy and Emissions by Variable Compression Ratio Using GT - Power," SAE Technical Paper 2010-01-1113, 2010. 
52. Allen J., "Introduction to Hardware-in-the-loop" (presentation at the EcoCAR Year 1 Fall Workshop, Boston, Massachusetts, September 27" 2008 ).

53. Modelica, "Modelica and the Modelica Association.” Accessed July 1, 2013, https://modelica.org/.

54. Modelica, "Modelica Libraries.” Accessed July 1, 2013, https://modelica.org/libraries.

55. Hendrickson J., Holmes A. and Freiman D., "General Motors Front Wheel Drive TwoMode Hybrid Transmission." SAE Technical Paper 2009-01-0508, 2009.

56. Rizzoni G., Guezennec Y., Brahma A., Wei X. and Miller T., "VP-SIM: A Unified Approach to Energy and Power Flow Modeling Simulation and Analysis of Hybrid Vehicles" (Proceedings of the 2000 Future Car Congress, Arlington, Virginia).

57. Heywood J., "Internal Combustion Engine Fundamentals," McGraw-Hill, New York, 1988.

58. Rizzoni G., Guzzella L. and Baumann B., "Modeling and Design Optimization of Hybrid Vehicles,” IEEE / ASME Transactions on Mechatronics 4 (1999): 246-257.

59. Wei X. and Rizzoni G., "A scalable approach for energy converter modeling and supervisory control design” (Proc. of ASME IMECE’2001, November 2001).

60. Rizzoni G, Guzzella L. and Baumann B., "Unified modeling of hybrid electric vehicle drivetrains," IEEE/ASME Transactions on Mechatronics 4(3) (1999): 246-257.

61. Rajagopalan A., Washington G., Rizzoni G., and GueZennec Y., "Development of fuzzy logic and neural network control and advanced emissions modeling for parallel hybrid vehicles," Subcontractor Report. December 2003, NREL/SR-540-32919.

62. Brugard J. and Bergstr"om J., "Modeling of a turbo charged spark ignited engine" (Master's thesis, Linkopings University, 1999).

63. Guzzella L. and Amstutz A., "Control of diesel engines," IEEE Transactions on Automatic Control 18(5) (1998): 53-71.

64. Marberg J., “Turbocharger for GT-power simulation.” Technical Report M17/577, 1999. Internal Scania report.

65. Muller M., "Mean value modeling of turbocharged spark ignition engines," SAE Technical Paper 980784, 1998.

66. Pettersson F., "Simulation of a turbo charged spark ignited engine." Master's thesis, Linkopings University, 2000.

67. dSPACE GMBH, "dSPACE ASM Diesel Engine reference for ASM Diesel Engine Blockset 1.6.1 and ASM Diesel Engine Operator Blockset 1.6.1," dSPACE R7.4 User Manual, 2012.

68. dSPACE GMBH, “dSPACE ASM Electric Components Reference for ASM Electric Components Blockset 2.3,” dSPACE R7.4 User Manual, November 2012.

69. Culcu H., Verbrugge B., Omar N., Van Den Bossche P. and Van Mierlo J., "Internal resistance of cells of lithium battery modules with FreedomCAR model," World Electric Vehicle Journal 3 (2009): 1-9.

70. Doerffel D. and Abu Sharkh S., "A critical review of using the Peukert equation for determining the remaining capacity of lead-acid and lithium-ion batteries," Journal of Power Sources 155 (2006): 395-400. 
71. "Introducing a new module for the EcoCAR battery packs" (EcoCAR year 2 fall workshop presentation, Natick, MA, Sept. 2009).

72. Genta, G., "Motor vehicle dynamics: modeling and simulation," Singapore: World Scientific Publishing Co. Pte. Ltd., 1997.

73. Toyota, “Toyota Technical Training: Section 2- Hybrid System Operation.” Accessed July 7, 2013, http://www.autoshop101.com/forms/Hybrid02.pdf.

74. Benford H. L. and Leising M. B., "The lever analogy: a new tool in transmission analysis," SAE Technical Paper 810102, 1981.

75. Arnett M. and Faucett J. "2-Mode control software overview" (EcoCAR year 2 winter workshop presentation, Daytona, FL, January, 2010).

76. Duoba M., Lohse-Busch H., Carlson R., Bohn T. and Gurski S., "Analysis of Power Split HEV Control Strategies Using Data from Several Vehicles." SAE Technical Paper 200701-0291, 2007.

77. Arnett M. and Faucett J., "2-Mode control software overview" (EcoCAR year 2 winter workshop presentation, Daytona, FL, January, 2010).

78. Analog Device, "Isolation, Frequency Input: 5B45/5B46.” Accessed January 19, 2013, http://www.analog.com/static/imported-files/data_sheets/5B45_46.pdf.

79. BRUSA Inc., "Battery charger.” Accessed July 17, 2013, http://www.brusa.biz/uploads/media/BRUSA_NLG513_datasheetV02_01.pdf.

80. Sasaki S., "Toyota's Newly Developed Hybrid Powertrain" (Paper presented at the Proceeding of 1998 International Symposium on Power Semiconductor Devices \& ICs, Kyoto, Japan, June 3-6, 1998).

81. Ahn K. and Papalambros P.Y., "Engine optimal operation lines for power split hybrid electric vehicles," Journal of Automobile Engineering 233(9) 2009: 1149-1162.

82. Ahn K., Cho S., Cha S.W. and Lee J.M., "Engine operation for the planetary gear hybrid powertrain," Journal of Automobile Engineering 220(12) 2006: 1727-1735.

83. Limnios N., "Fault Trees", ISTE USA, 2007.

84. Amberkar S., Kushion M., Eschtruth K. and Bolourchi, F., "Diagnostic Development for an Electric Power Steering System," SAE Technical Paper, 2000-01-0819, 2000. 